\title{
Get moving! Self-management support using mobile technology : a monitoring and feedback tool embedded in a counselling protocol to increase physical activity of patients with COPD or type 2 diabetes in primary care: the It's LiFe! study
}

Citation for published version (APA):

van der Weegen, S. (2015). Get moving! Self-management support using mobile technology : a monitoring and feedback tool embedded in a counselling protocol to increase physical activity of patients with COPD or type 2 diabetes in primary care: the It's LiFe! study. [Doctoral Thesis, Maastricht University]. Maastricht University. https://doi.org/10.26481/dis.20150916sw

\section{Document status and date:}

Published: 01/01/2015

DOI:

10.26481/dis.20150916sw

\section{Document Version:}

Publisher's PDF, also known as Version of record

\section{Please check the document version of this publication:}

- A submitted manuscript is the version of the article upon submission and before peer-review. There can be important differences between the submitted version and the official published version of record. People interested in the research are advised to contact the author for the final version of the publication, or visit the DOI to the publisher's website.

- The final author version and the galley proof are versions of the publication after peer review.

- The final published version features the final layout of the paper including the volume, issue and page numbers.

Link to publication

\footnotetext{
General rights rights.

- You may freely distribute the URL identifying the publication in the public portal. please follow below link for the End User Agreement:

www.umlib.nl/taverne-license

Take down policy

If you believe that this document breaches copyright please contact us at:

repository@maastrichtuniversity.nl

providing details and we will investigate your claim.
}

Copyright and moral rights for the publications made accessible in the public portal are retained by the authors and/or other copyright owners and it is a condition of accessing publications that users recognise and abide by the legal requirements associated with these

- Users may download and print one copy of any publication from the public portal for the purpose of private study or research.

- You may not further distribute the material or use it for any profit-making activity or commercial gain

If the publication is distributed under the terms of Article $25 \mathrm{fa}$ of the Dutch Copyright Act, indicated by the "Taverne" license above, 


\section{Get Moving!}

Self-management support using mobile technology

A monitoring and feedback tool embedded in a counselling protocol to increase physical activity of patients with COPD or type 2 diabetes in primary care: the It's LiFe! study
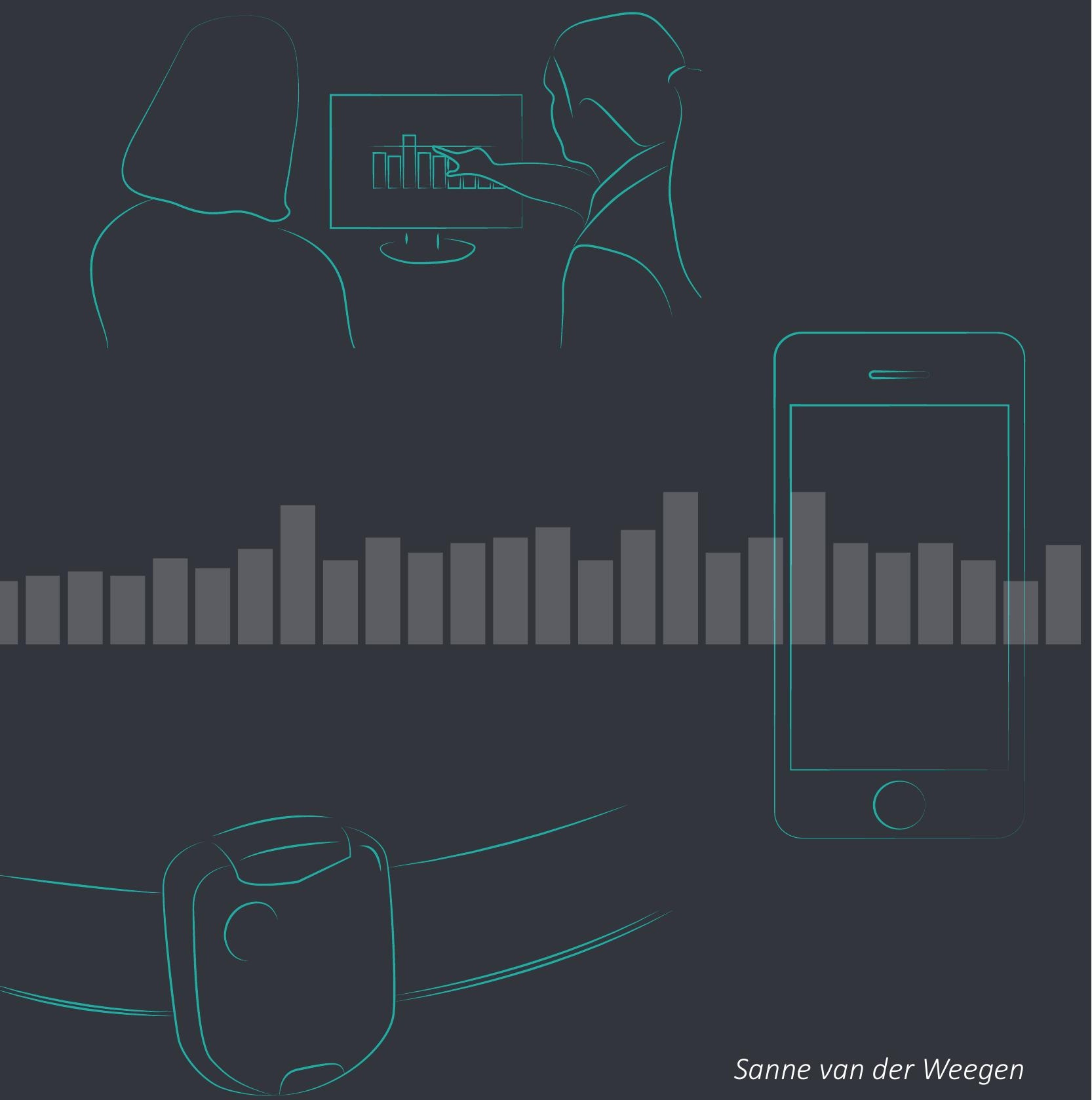
The studies presented in this dissertation were performed at the Department of Health Services Research, School for Public Health and Primary Care (CAPHRI), Maastricht University. CAPHRI is part of the Netherlands School of Primary Care Research (CaRe), which has been acknowledged since 1995 by the Royal Netherlands Academy of Arts and Sciences (KNAW).

The studies described in this dissertation are all part of the research project Interactive Tool for Selfmanagement through Lifestyle Feedback! (It's LiFe!). This project was funded by the programme 'Disease management chronical illnesses'. Through this program the Netherlands Organisation for Health Research and development (ZonMw) and the Netherlands Organisation for Scientific Research (NWO) want to promote the development of new technology for healthcare. At a later stage, the theme 'New Instruments for Healthcare (NIG) was added to this program.

The companies involved in the development of the technology were:

- IDEE Maastricht UMC+ Universiteitssingel 50, 6229 ER Maastricht, the Netherlands, www.idee-mumc.nl

- Maastricht Instruments Ltd. Oxfordlaan 70, 6229 EV Maastricht, the Netherlands, www.maastrichtinstruments.nl

- Sananet Care Ltd. Rijksweg Zuid 22A, 6131 AP Sittard, the Netherlands, www.sananet.nl

Printing and dissimination of this dissertation was financially supported by the following companies: Maastricht Instruments Ltd, Sananet Care Ltd. and PAM Ltd.

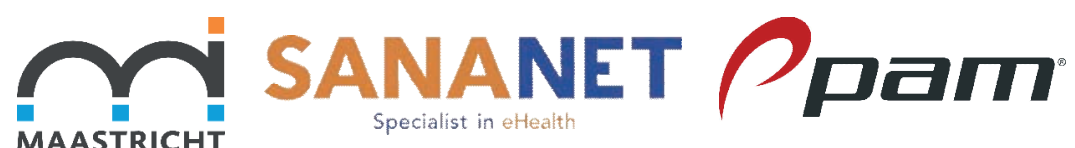

INSTRUMENTS

Design cover: Lotte Cornelisse

(C) copyright Sanne (J.C.M.) van der Weegen, Maastricht 2015

Printing: Datawyse | Universitaire Pers Maastricht

ISBN 9789461594600 


\section{Get moving!}

\section{Self-management support using mobile technology}

A monitoring and feedback tool embedded in a counselling protocol to increase physical activity of patients with COPD or type 2 diabetes in primary care:

the It's LiFe! study

PROEFSCHRIFT

ter verkrijging van de graad van doctor

aan de Universiteit Maastricht

op gezag van de Rector Magnificus, Prof. Dr. L.L.G. Soete,

volgens het besluit van het College van Decanen,

in het openbaar te verdedigen

op woensdag 16 september 2015 om 14.00 uur

door

Sanne van der Weegen

Geboren op 25 november 1986 te Steenbergen

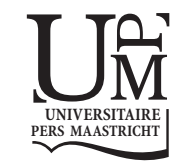




\section{Promotor}

Prof. dr. L.P. de Witte

\section{Copromotores}

Dr. M.D. Spreeuwenberg

Dr. H.J. Tange

\section{Beoordelingscommissie}

Prof. dr. J. Muris (voorzitter)

Prof. dr. ir. R.D. Friele (Tilburg University)

Prof. dr. ir. H.J. Hermens (University of Twente)

Prof. dr. S. Kremers

Prof. dr. D. Ruwaard 


\section{CONTENTS}

$\begin{array}{lll}\text { Chapter } 1 & \text { General introduction } & 7\end{array}$

Chapter 2 The development of a mobile monitoring and feedback tool to 19 stimulate physical activity of people with a chronic disease in primary care: A User-Centred Design

Chapter 3 Usability testing of a monitoring and feedback tool to stimulate physical activity

Chapter 4 Concurrent validity of the MOX activity monitor compared to the 61 ActiGraph GT3X

Chapter 5 Concurrent validity of the Pam AM300 accelerometer relative to the 79 ActiGraph GT3X

Chapter 6 A monitoring and feedback tool embedded in a counselling protocol 93 to increase physical activity of patients with COPD or type 2 diabetes in primary care: study protocol of a three-arm cluster randomised controlled trial

Chapter 7 It's LiFe! mobile and web-based monitoring and feedback tool embedded in primary care Increases physical activity: a cluster randomised controlled trial

Chapter 8 Process evaluation of physical activity counselling with and without 135 the use of mobile technology: a mixed methods study

Chapter 9 General discussion 159

$\begin{array}{lll}\text { Chapter } 10 \quad \text { Valorisation } & 175\end{array}$

$\begin{array}{ll}\text { Summary } & 181\end{array}$

$\begin{array}{ll}\text { Samenvatting } & 189\end{array}$

Dankwoord 195

$\begin{array}{ll}\text { About the author } & 199\end{array}$

List of publications $\quad 201$ 

CHAPTER 1

General introduction 

Mrs. Brown (55 years old) has always been a bit too heavy, she knew she would never become the next Miss Universe, but she never thought about the health consequences it could have. Since she was diagnosed with type 2 diabetes a lot has changed. She cannot just unconsciously enjoy her beloved self-made chocolate cakes or stews anymore. On a daily basis, she has to take blood-sugar lowering medication and inject insulin. The primary care providers assist her in her new role as a patient. Mrs. Brown is now used to take the medication and she changed her diet a bit. However the advice to be more physically active is more difficult to follow.

Last week Mrs. Brown had an appointment with her practice nurse, who asked her what the progress was regarding her daily physical activity. Mrs. Brown had to admit that she was not active enough. She did subscribe to a fitness school in her neighbourhood, but she only went a few times. Her fulltime job, three children and her ill mother do take up too much of her time. She really had good intentions, but ultimately she did not manage to find the motivation and the time to keep going to the gym. The practice nurse therefore suggests supporting Mrs. Brown more actively to become more active and she opted to use a monitoring tool for support on a daily basis.

The It's LiFe! project was initiated to support people like Mrs. Brown in their physical activity behaviour change. The project combines two interventions to enhance physical activity: a monitoring \& feedback tool for daily support of the patients and a counselling protocol to be followed by the practice nurse. Two theses are produced during the It's LiFe! project. The aim of this dissertation is to describe the development, testing and evaluation of the It's LiFe! monitoring and feedback system embedded in primary care, which is based on mobile technology and fulfils the needs and preferences of the end users. In addition, two accelerometers were validated.

\section{Physical (in) activity}

Having 30 minutes of moderate to vigorous intensity physical activity on at least 5 days throughout the week is beneficial for the health of the entire population. ${ }^{1-4}$ Some organisation have adapted guidelines by recommending 20 minutes of vigorous-intensity physical activity on at least 3 days of the week, or an equivalent combination achieving 600 metabolic equivalent (MET)-min per week. ${ }^{2-5}$ The MET expresses the energy spent during physical activities as a multiple of the resting metabolic rate. ${ }^{6}$ MET-minutes are calculated by multiplying the minutes engaged in an activity by the number of METS of that activity. ${ }^{2-4}$ People who are insufficiently active have a $20 \%$ to $30 \%$ increased risk of death compared to people who do meet the recommended levels of physical activity. ${ }^{7}$ Next to reduced rates of mortality, regular physical activity leads to reduced rates of type 2 diabetes, stroke, coronary heart disease, breast and colon cancers and depression. ${ }^{8,9}$ Additionally, physical activity leads to improved bone health, increased functional health, increased cardio-respiratory and muscular fitness, healthier body mass and com- 
position, and improved cognitive function. ${ }^{8,9}$ Despite these health benefits, worldwide $31.1 \%$ of the adults above 15 years old are still physically inactive. ${ }^{6}$

For people with a chronic disease it is harder to be sufficiently active than for healthy people. People with Chronic Obstructive Pulmonary Disease (COPD) are rapidly exhausted during physical activity due to dyspnea. Type 2 diabetic patients often are overweight or obese, which makes physical activity more difficult. ${ }^{10,11}$ This leads to a vicious cycle of less physical activity and increasing discomfort during physical activity. ${ }^{10,11}$ The prevalence of physical inactivity in people with COPD and type 2 diabetes is therefore higher than in healthy individuals, according to a Swedish study respectively $84 \%$ and $73 \%{ }^{12}$

Among people with COPD, physical inactivity holds the risk of reduced health related quality of life ${ }^{13}$ and increase of COPD related hospital admission and respiratory mortality. ${ }^{14}$ In fact, physical activity is the strongest predictor of all-cause mortality for people with COPD. ${ }^{15}$

People with type 2 diabetes mellitus have an inadequate insulin secretion, because the body has developed a resistance to insulin. ${ }^{16}$ Among people with type 2 diabetes, regular physical activity will reduce the incidence of cardiovascular-related mortality and all-cause premature death. In addition, a review of Ivy shows that regular physical activity (independent of changes in body mass) improves glucose homeostasis, due to improvements in the quantity and distribution of the glucose transporter protein GLUT 4, improvement in the blood flow of the muscles (resulting in enhanced glucose delivery to the muscle) and increased glycogen synthase. ${ }^{17,18}$ One week of moderate to vigorous aerobic physical activity or exercise can already positively change overall body insulin sensitivity. ${ }^{19-21}$

\section{Physical activity promotion in primary care}

Given the numerous risks of physical inactivity and the subsequent economic burden on healthcare systems, promotion of physical activity has become a major health challenge. A behaviour change has to be accomplished in inactive people. For people with a chronic disease, one of the approaches to decrease the prevalence of physical inactivity is through primary health care. ${ }^{3,22}$ Primary care is well placed to promote physical activity, because people with chronic conditions visit the family practice frequently. It is recommended that primary care providers incorporate support to change physical inactivity behaviours. ${ }^{23,24}$ Physical activity can be promoted in primary care in different ways, including provision of written materials, delivery of advice and referral to an exercise programme. ${ }^{25}$ However, conflicting evidence exists on the effectiveness of primary carebased physical activity interventions. ${ }^{26-28,29}$ There is room for improvement: a review by Hébert et al. revealed that most primary care providers believe that they have a role in promoting physical activity among their patients. However, they are uncertain about the 
effectiveness of counselling, feel uncomfortable in providing advice about physical activity and they experience a lack of time, training and reimbursement as barriers. ${ }^{30}$ A more recent review confirmed that lack of time and training were factors that negatively influenced physical activity promotion. This review indicated the professionals' perception of patients' lack of motivation to be physically active as an additional barrier.

As health care systems are historically developed to treat acute illnesses ${ }^{31}$ it is not surprising that barriers are encountered in coaching patients in the management of their chronic disease and behaviour change. The Chronic Care Model is a framework to guide quality improvements in chronic illness care. ${ }^{31}$ It states that the essential six elements for the encouragement of high-quality chronic care provision are the community, the healthcare system, self-management support, an appropriately organized delivery system design, clinical information systems, and decision support. ${ }^{31}$ Effective selfmanagement support and links to patient-oriented community resources help to activate and inform patients and their relatives to better cope with the challenges of living with and chronic condition. ${ }^{31}$

\section{Self-management}

Although Mrs. Brown is happy to be supported by her practice nurse, in daily living she has to deal with barriers to become active by herself. Because support from care providers is not infinite and patients are responsible for the management of their own disease, they need skills to manage their disease every day. ${ }^{32}$ Self-management refers to the tasks that an individual must undertake to live well with one or more chronic conditions. This incorporates gaining confidence to deal with medical management, role management, emotional management ${ }^{33}$, but also taking the lead over the care process. ${ }^{34}$ Three conditions for optimal self-management for patients are 1) sufficient knowledge and skills 2) care professionals who support self-management and provide a safe environment to gain experience with the 'management' of their disease or disability and 3) insight in their disease and behaviours. ${ }^{34}$ Therefore self-management is a central element in most chronic disease management programs as well as in the Chronic Care Model. Physical activity is a typical behaviour which depends on the self-management skills of a patient. The task of the care provider is to optimally support this selfmanagement. Self-management support is defined as the systematic provision of education and supportive interventions by health care staff to increase patients' skills and confidence in managing their health problems, including regular assessment of progress and problems, goal setting, and problem-solving support. ${ }^{33}$ Supporting people in their self-management is more than giving advice, it is building on what they are already doing. $^{35}$ 


\section{Physical activity promotion and technology}

Technology is a promising platform in creating the third condition for effective selfmanagement, insight in the disease or behaviour, by making remote self-monitoring and personalized feedback automated and easy. ${ }^{36}$

Monitoring and feedback tools are rapidly entering the consumer market such as pedometers, accelerometers and heart rate monitors. Furthermore, the fast diffusion of smartphones and tablets provides a platform for many mobile health applications (apps). ${ }^{37}$

Pedometers are small, relatively inexpensive devices that count and monitor the number of steps taken throughout the day. A review by Bravata et al. in outpatient adults shows that the use of a pedometer is associated with significant increases in physical activity and significant decreases in body mass index and blood pressure. ${ }^{38}$ However, there is no evidence if changes sustain over the long term. Three dimensional accelerometers are more advanced. Accelerometers measure body accelerations minute to minute and provide detailed information on frequency, duration, intensity and pattern of movement. ${ }^{39}$ Counts from accelerometers are mostly used to estimate energy expenditure. ${ }^{39,40}$ Heart rate monitors are mainly used to monitor exercise intensity and give an indication of exercise energy expenditure. However these estimates are affected by factors such as temperature, hydration and emotional stress. ${ }^{40}$ Therefore accelerometers and heart rate monitors are sometimes used together. This improves the prediction of exercise energy expenditure by $20 \% .{ }^{40,41}$ Together with these wearable devices specifically targeted at measuring physical activity or energy expenditure, applications (apps) on smartphones and tablets are becoming more and more popular to measure and process these measurements in terms of health outcomes. ${ }^{42}$ In 2012, it was estimated that half of the smartphone owners in the U.S. use their device to get health information and one-fifth of the owners have health apps. ${ }^{43}$ This was twice as much as in 2010. Exercise, diet and weight apps are the most popular. ${ }^{43}$

Although these developments are highly encouraged, many of the commercially available tools and apps are not validated nor based on established health behavioural theories. ${ }^{42}$ In addition, these tools and apps are merely targeted and used by healthy consumers who are already enthusiastic about physical activity and are motivated to share their activity results with friends, to make it a challenge or push themselves harder. Our aim is different: we want to involve people with chronic conditions who are in need of a supportive health context. Recent research also suggests that telehealthcare is most successful if integrated with education and intensive support. ${ }^{36}$ The supportive context could be established by the practice nurse in primary care since the majority of chronically ill people visit the practice nurse regularly and lifestyle counselling is part of the responsibility of the practice nurse.

We do believe that the combination of counselling in primary care and the use of technology reinforces best of both worlds. The practice nurse can generate awareness of 
the risks of physical inactivity and be the necessary supportive context. Technology can in turn support the patient in daily living with providing real time feedback, but also provide the care professional with more accurate and frequent information about the behaviour of the patient. This will ultimately improve self-management support.

\section{Theoretical background for physical activity promoting intervention}

To develop an effective intervention to change behaviour it is recommended to base the intervention on health behaviour theories, because these help us to understand the determinants of behaviour that needs to be influenced to encourage a person to change its behaviour. Moreover, it assists in choosing relevant behaviour change techniques. ${ }^{27,44,45}$ Several behavioural theories and models are adapted and used for the understanding of physical activity behaviour change and to design effective interventions. ${ }^{46}$ These theories and models generally include: the classical learning theory, the health belief model; ${ }^{47}$ the transtheoretical model, ${ }^{48-50}$ relapse prevention, social cognitive theory, theory of reasoned action and planned behaviour, ${ }^{43}$ social support, selfregulation theory, and ecological approaches. ${ }^{46,51}$ These theories can be translated into behaviour change techniques. Behaviour change techniques that have proven to be effective in the promotion of physical activity are related to the conditions required for effective self-management. These techniques are: self-monitoring, providing feedback of behaviour, goal setting, provision of tools to facilitate behaviour, action planning, social support, barrier identification, and providing information on the consequences specific to the individual. ${ }^{27,52,53}$

\section{User involvement}

Besides integrating the right elements and having a theoretical foundation, another prerequisite for a successful intervention is the involvement of end users in the development process. ${ }^{54}$ Both for the development of the technology and the development of the primary care component patients and care providers can provide valuable information. If experienced barriers of patients to become active and barriers of care providers for supporting patients in their self-management are known, the intervention can be tailored. Not only information about barriers, but also first-hand knowledge about facilitators and context is valuable. It is important to know what patients motivate, what activity data is of interest for patients and care providers, what mode of delivery fits in their lives and daily routines, if and with whom they would like to share data, how many contacts between care provider and patient are desirable and which care provider is most appropriate. Involving end users needs, expectations, experiences and requirements will improve the usability of the technology and acceptability of the complete 
intervention. ${ }^{54,55}$ In this project an iterative User-centred design approach was used to involve user needs and requirements in the development of a monitoring and feedback tool and a counselling protocol for primary care. 'User-centred design' is a broad term originated from Human-Computer Interaction, which indicates that users are involved in the design process, one way or another. ${ }^{56,57}$ In the It's LiFe! project there was a close collaboration between the research team and an engineering team. The research team gathered user requirements from end users by interviews and focus groups and two patient's representatives were partners in the research team throughout the development and evaluation process.

\section{Aims and outline of the dissertation}

The main objective of this dissertation is to study whether a monitoring and feedback tool in combination with a counselling protocol in primary care leads to higher levels of physical activity in people with COPD or type 2 diabetes.

The underlying objectives for this were to:

1. Develop a monitoring and feedback tool, which fulfils the needs and preferences of patients and care professionals and can be embedded in a counselling protocol.

2. Test the usability of the tool in a laboratory setting and in daily living.

3. Develop appropriate thresholds for the moderate and vigorous activity categories for the activity monitor of the It's LiFe! tool.

4. Test the concurrent validity of the activity monitor of the It's LiFe! tool and a low-cost commercially available activity monitor, compared to a state of the art activity monitor the ActiGraph GT3X.

5. Evaluate the effectiveness of the counselling protocol with and without the use of the tool in a cluster randomised controlled trial.

6. Conduct a process evaluation in parallel with the trial to examine the reach, implementation and satisfaction regarding this counselling protocol with and without the use of the tool.

In addition, in a second dissertation based on the It's LiFe! project, written by colleague Renée Verwey, the research questions related to objectives five and six are identical and jointly examined. Her dissertation specifically focuses on the development and testing of the counselling protocol and the coaching system for the practice nurses, whereas in this dissertation the focus is on the development and testing of the monitoring and feedback tool and on the validation of two accelerometers.

The project started with a user centred development process in which the monitoring and feedback tool and the counselling protocol (Self-management Support Programme) based on the principles of the Chronic Care Model were developed. Chapter 2 describes the iterative user centred development process of the monitoring and feedback tool which was a close collaboration between the researchers and an engineering 
team with input from patients, care professionals and experts. This study resulted in the It's LiFe! tool which consists of a 3D activity monitor, a smartphone app and a web application for patients and their practice nurse. Chapter 3 presents the results of the usability study of the tool. Patients were observed while using the tool in a laboratory setting and experiences with the use of the tool were gathered during a three month pilot in daily living.

Chapter 4 reports on the concurrent validity of the MOX activity monitor, part of the It's LiFe! tool, relative to the ActiGraph GT3X. Chapter 5 presents the results of a similar study where the Pam AM300, the primary outcome of the effect study, is evaluated against the ActiGraph GT3X.

The design of the cluster randomized controlled trial (RCT) and the process evaluation, which was conducted simultaneously with the RCT, are outlined in Chapter 6. Chapter 6 also describes the inclusion and exclusion criteria of participants, the recruitment of general practices and participants, the randomization procedure, the content of the intervention, data collection and data analyses of the RCT and process evaluation. Chapter 7 reports the results of the RCT and answers the main research question of the dissertation. It was investigated if the It's LiFe! monitoring and feedback tool embedded in the Self-management Support Programme (SSP) was of added value compared to care as usual and what the effect was of execution of the SSP compared to care as usual. Results are presented on minutes of moderate to vigorous physical activity per day (primary outcome), quality of life, general self-efficacy and exercise self-efficacy. How the interventions were carried out, which patients were reached and how satisfied practice nurses and participants were with the interventions in the RCT is described in the process evaluation, presented in Chapter 8.

A general discussion about the six studies, including the limitations and implications for practice, future research and policy, extracted from this dissertation, are described in Chapter 9.

Finally, Chapter 10 explains how the results from this dissertation can be used and implemented in practice.

\section{References}

1. Wendel-Vos G. Normen lichamelijke (in)activiteit. Volksgezondheid Toekomst Verkenning, Nationaal Kompas Volksgezondheid 2014;

http://www. nationaalkompas.nl/gezondheidsdeterminanten/leefstij//lichamelijke-activiteit/normen-vanlichamelijke-in-activiteit/. Accessed 21 July, 2014.

2. Hallal PC, Andersen LB, Bull FC, Guthold R, Haskell W, Ekelund U. Global physical activity levels: surveillance progress, pitfalls, and prospects. Lancet. Jul 21 2012;380(9838):247-257.

3. Organisation WH. Global recommendations on physical activity for health. In: Organisation WH, ed. Geneva2010.

4. WHO. Global Physical Activity Questionnaire (GPAQ) Analysis Guide. http://www.webcitation.org/6SMZ1grOC. Accessed 5 September 2014, 2014. 
5. Haskell WL, Lee IM, Pate RR, et al. Physical activity and public health: updated recommendation for adults from the American College of Sports Medicine and the American Heart Association. Circulation. Aug 28 2007;116(9):1081-1093.

6. Ainsworth BE, Haskell WL, Herrmann SD, et al. 2011 Compendium of Physical Activities: a second update of codes and MET values. Med Sci Sports Exerc. Aug 2011;43(8):1575-1581.

7. WHO. Physical activity fact sheet $N^{0} 385$. 2014; http://www.webcitation.org/6SMZsQsXZ Accessed 5 september 2014, 2014.

8. Lee IM, Shiroma EJ, Lobelo F, Puska P, Blair SN, Katzmarzyk PT. Effect of physical inactivity on major noncommunicable diseases worldwide: an analysis of burden of disease and life expectancy. Lancet. Jul 21 2012;380(9838):219-229.

9. Warburton DE, Charlesworth S, Ivey A, Nettlefold L, Bredin SS. A systematic review of the evidence for Canada's Physical Activity Guidelines for Adults. The international journal of behavioural nutrition and physical activity. 2010;7:39.

10. Troosters $\mathrm{T}$, van der Molen T, Polkey M, et al. Improving physical activity in COPD: towards a new paradigm. Respiratory research. 2013;14:115.

11. Cooper CB. Airflow obstruction and exercise. Respiratory medicine. Mar 2009;103(3):325-334.

12. Arne $M$, Janson $C$, Janson $S$, et al. Physical activity and quality of life in subjects with chronic disease: chronic obstructive pulmonary disease compared with rheumatoid arthritis and diabetes mellitus. Scand J Prim Health Care. 2009;27(3):141-147.

13. Durr S, Zogg S, Miedinger D, Steveling EH, Maier S, Leuppi JD. Daily physical activity, functional capacity and quality of life in patients with COPD. COPD. Dec 2014;11(6):689-696.

14. Garcia-Aymerich J, Lange P, Benet M, Schnohr P, Anto JM. Regular physical activity reduces hospital admission and mortality in chronic obstructive pulmonary disease: a population based cohort study. Thorax. Sep 2006;61(9):772-778.

15. Waschki B, Kirsten A, Holz O, et al. Physical activity is the strongest predictor of all-cause mortality in patients with COPD: a prospective cohort study. Chest. Aug 2011;140(2):331-342.

16. Berkow R, Beers MH, Fletcher AJ. The Merck manual of medical information. Whitehouse Station, N.J.: Merck Research Laboratories; 1997.

17. Warburton DE, Nicol CW, Bredin SS. Health benefits of physical activity: the evidence. Canadian medical association journal. 2006;174(6):801-809.

18. IVy JL. Role of exercise training in the prevention and treatment of insulin resistance and non-insulindependent diabetes mellitus. Sports Med. Nov 1997;24(5):321-336.

19. Durstine JL, Gordon B, Wang Z, Luo X. Chronic disease and the link to physical activity. Journal of Sport and Health Science. 2013;2(1):3-11.

20. Winnick JJ, Sherman WM, Habash DL, et al. Short-term aerobic exercise training in obese humans with type 2 diabetes mellitus improves whole-body insulin sensitivity through gains in peripheral, not hepatic insulin sensitivity. J Clin Endocrinol Metab. Mar 2008;93(3):771-778.

21. Colberg SR, Albright AL, Blissmer BJ, et al. Exercise and type 2 diabetes: American College of Sports Medicine and the American Diabetes Association: joint position statement. Exercise and type 2 diabetes. Med Sci Sports Exerc. Dec 2010;42(12):2282-2303.

22. NCD prevention: investments [corrected] that work for physical activity. Br J Sports Med. Aug 2012;46(10):709-712.

23. Peterson JA. Get moving! Physical activity counselling in primary care. J Am Acad Nurse Pract. Jul 2007;19(7):349-357.

24. Heiligers PJM, Noordman, J, Korevaar, JC, Dorsman, S, Hingstman, L, Dulmen, A.M. van, Bakker, DH de. Kennisvraag: praktijkondersteuners in de huisartspraktijk (POH's) klaar voor de toekomst? Utrecht: Nivel;2012.

25. Orrow G, Kinmonth AL, Sanderson S, Sutton S. Effectiveness of physical activity promotion based in primary care: systematic review and meta-analysis of randomised controlled trials. Bmj. 2012;344:e1389.

26. Eakin EG, Glasgow RE, Riley KM. Review of primary care-based physical activity intervention studies: effectiveness and implications for practice and future research. J Fam Pract. Feb 2000;49(2):158-168. 
27. Avery L, Flynn D, van Wersch A, Sniehotta FF, Trenell MI. Changing physical activity behaviour in type 2 diabetes: a systematic review and meta-analysis of behavioural interventions. Diabetes care. Dec 2012;35(12):2681-2689.

28. Hillsdon M, Foster C, Thorogood M. Interventions for promoting physical activity. Cochrane Database Syst Rev. 2005(1):CD003180.

29. Williams NH. "The wise, for cure, on exercise depend": physical activity interventions in primary care in Wales. BrJ Sports Med. Feb 2009;43(2):106-108.

30. Hebert ET, Caughy MO, Shuval K. Primary care providers' perceptions of physical activity counselling in a clinical setting: a systematic review. Br J Sports Med. Jul 2012;46(9):625-631.

31. Wagner EH, Austin BT, Davis C, Hindmarsh M, Schaefer J, Bonomi A. Improving chronic illness care: translating evidence into action. Health Aff (Millwood). Nov-Dec 2001;20(6):64-78.

32. Bodenheimer T, Lorig K, Holman H, Grumbach K. Patient self-management of chronic disease in primary care. Jama. Nov 20 2002;288(19):2469-2475.

33. Adams K, Greiner A, Corrigan J. Report of a summit. The 1st annual crossing the quality chasm summit: a focus on communities. 2004:6-7.

34. Brink Rvd, Timmermans H, Havers J, Veenendaal Hv. Ruimte voor regie: pioniers over zelfmanagement in de zorg. Deventer: Kluwer; 2013.

35. longh Ad. Patients need support to self manage their long term conditions. BMJ: British Medical Journal. 2014;348.

36. McLean S, Sheikh A, Cresswell K, et al. The impact of telehealthcare on the quality and safety of care: a systematic overview. PLoS One. 2013;8(8):e71238.

37. West JH, Hall PC, Hanson CL, Barnes MD, Giraud-Carrier C, Barrett J. There's an app for that: content analysis of paid health and fitness apps. J Med Internet Res. 2012;14(3):e72.

38. Bravata DM, Smith-Spangler C, Sundaram V, et al. Using pedometers to increase physical activity and improve health: a systematic review. Jama. Nov 21 2007;298(19):2296-2304.

39. Plasqui G, Westerterp KR. Physical Activity Assessment With Accelerometers: An Evaluation Against Doubly Labeled Water. Obesity. 2007;15(10):2371-2379.

40. Heyward VH, Gibson AL. Advanced fitness assessment and exercise prescription. Champaign, IL: Human Kinetics; 2014.

41. Strath SJ, Brage S, Ekelund U. Integration of physiological and accelerometer data to improve physical activity assessment. Med Sci Sports Exerc. Nov 2005;37(11 Suppl):S563-571.

42. Cowan LT, Van Wagenen SA, Brown BA, et al. Apps of steel: are exercise apps providing consumers with realistic expectations?: a content analysis of exercise apps for presence of behaviour change theory. Health Educ Behav. Apr 2013;40(2):133-139.

43. Fox S, Duggan M. Mobile health 2012. Washington, DC: Pew Internet \& American Life Project. 2012.

44. Webb TL, Joseph J, Yardley L, Michie S. Using the Internet to Promote Health Behaviour Change: A Systematic Review and Meta-analysis of the Impact of Theoretical Basis, Use of Behaviour Change Techniques, and Mode of Delivery on Efficacy. J Med Internet Res. 2010;12(1):e4.

45. Bully P, Sanchez A, Zabaleta-Del-Olmo E, Pombo H, Grandes G. Evidence from interventions based on theoretical models for lifestyle modification (physical activity, diet, alcohol and tobacco use) in primary care settings: A systematic review. Preventive medicine. Jan 52015.

46. Tudor-Locke $C$, Lutes $L$. Why do pedometers work?: a reflection upon the factors related to successfully increasing physical activity. Sports Med. 2009;39(12):981-993.

47. Janz NK, Becker MH. The Health Belief Model: a decade later. Health Educ Q. Spring 1984;11(1):1-47.

48. Prochaska JO, DiClemente CC. Stages and processes of self-change of smoking: toward an integrative model of change. J Consult Clin Psychol. 1983;51(3):390.

49. Prochaska JO, Velicer WF. The transtheoretical model of health behaviour change. Am J Health Promot. Sep-Oct 1997;12(1):38-48.

50. Biddle SJH, Mutrie N. Psychology of Physical Activity: Determinants, well-being and interventions. second ed. London: Routledge; 2008. 
51. General USPHSOotS, Control CfD, Prevention NCfCD, Promotion H, Fitness PsCoP, Sports. Physical activity and health: a report of the Surgeon General: Jones \& Bartlett Learning; 1996.

52. Artinian NT, Fletcher GF, Mozaffarian D, et al. Interventions to promote physical activity and dietary lifestyle changes for cardiovascular risk factor reduction in adults: a scientific statement from the American Heart Association. Circulation. Jul 27 2010;122(4):406-441.

53. van Achterberg T, Huisman-de Waal GG, Ketelaar NA, Oostendorp RA, Jacobs JE, Wollersheim HC. How to promote healthy behaviours in patients? An overview of evidence for behaviour change techniques. Health Promot Int. Jun 2011;26(2):148-162.

54. Shah SG, Robinson I, AlShawi S. Developing medical device technologies from users' perspectives: a theoretical framework for involving users in the development process. Int J Technol Assess Health Care. Oct 2009;25(4):514-521.

55. Shah SG, Robinson I. Benefits of and barriers to involving users in medical device technology development and evaluation. Int J Technol Assess Health Care. Winter 2007;23(1):131-137.

56. Abras C, Maloney-Krichmar D, Preece J. User-centred design. Thousand Oaks: Sage; 2004.

57. Dabbs Ade V, Myers BA, Mc Curry KR, et al. User-centred design and interactive health technologies for patients. Comput Inform Nurs. May-Jun 2009;27(3):175-183. 
CHAPTER 2

The development of a mobile monitoring and feedback tool to stimulate physical activity of people with a chronic disease in primary care: A User-Centred Design

This chapter was published as:

van der Weegen S, Verwey R, Spreeuwenberg M, Tange $H$, van der Weijden $T$, de Witte L. The Development of a Mobile Monitoring and Feedback Tool to Stimulate Physical Activity of People with a Chronic Disease in Primary Care: A User-Centred Design. JMIR mhealth and uhealth. 2013;1(2):e8 


\section{Abstract}

Background Physical activity is an important aspect in the treatment of patients with chronic obstructive pulmonary disease or type-2 diabetes. A monitoring and feedback tool combined with guidance by a primary care provider might be a successful method to enhance the level of physical activity in these patients. As a prerequisite for useful technology, it is important to involve the end users in the design process from an early stage.

Objective The aim of this study was to investigate the user requirements for a tool to stimulate physical activity, embedded in primary care practice. The leading principle of this tool is to change behaviour by self-monitoring, goal-setting, and feedback.

Methods The research team collected qualitative data among 15 patients, 16 care professionals, and several experts. A prototype was developed in three stages. In stage 1, the literature was searched to identify end users and context. In stage 2, the literature, experts and patient representatives were consulted to set up a use case with the general idea of the innovation. In stage 3, individual interviews and focus groups were held to identify the end user requirements. Based on these requirements a prototype was built by the engineering team.

Results The development process has led to a tool that generally meets the requirements of the end users. A tri-axial activity sensor, worn on the hip, is connected by Bluetooth to a smartphone. In an app, quantitative feedback is given about the amount of activity and goals reached by means of graphical visualization, and an image shows a sun when the goal is reached. Overviews about activity per half an hour, per day, week, and month are provided. In the menu of the app and on a secured website, patients can enter information in individual sessions or read feedback messages generated by the system. The practice nurse can see the results of all patients on a secure webpage and can then discuss the results and set personalized goals in consultation with the patient.

Conclusions This study demonstrates that a user-centred approach brings in valuable details (such as the requirements for feedback in activity minutes per day) to improve the fit between the user, technology, and the organization of care, which is important for the usability and acceptability of the tool. The tool embedded in primary care will be evaluated in a randomized controlled trial. 


\section{Introduction}

Lack of physical activity is an important risk factor for cardiovascular disease, hypertension, diabetes mellitus, obesity, stroke, some cancers, and osteoporosis. It is recommended that the general population is physically active at a moderate to vigorous intensity for at least 150 minutes per week. ${ }^{1}$ Unfortunately, physical inactivity remains highly prevalent. ${ }^{2,3}$ It is particularly important for people with a chronic disease to be physically active. It has not only been proven that an active lifestyle prevents diseases but also an active lifestyle improves the health-related quality of life and psychological status for people with a chronic disease. ${ }^{4,5}$ An active lifestyle reduces dyspnea in chronic obstructive pulmonary disease (COPD) patients ${ }^{6}$ and complications in patients with diabetes. ${ }^{7}$ Due to the health benefits and the need for support by most COPD and type-2 diabetes (DM2) to increase their physical activity, stimulating physical activity is regarded as one of the main treatment goals in primary care. ${ }^{8,9}$ This should be accomplished with support for self-management. Self-management implies that people are in charge of their own lives with their disease and its treatment, enabling motivation to change. Supporting self-management requires a different role of health care professionals and patients, for which new skills and tools are needed. ${ }^{10}$

Professionals can be more successful at improving an active lifestyle by increasing patients' awareness through self-monitoring, goal setting, and discussing selfefficacy. ${ }^{11,12}$ The provision of tailored feedback on physical activity has been proven to be effective in several interventions. ${ }^{13-15}$ Persuasive technology can help professionals in accomplishing their coaching role. A "simple" pedometer gives feedback about the frequency of steps or distance walked in a day and it seems to be a useful tool that incorporates elements for self-monitoring, goal-setting, and feedback. Self-reporting studies revealed that the use of pedometers is an effective approach to increase physical activity. ${ }^{16-18}$ It is, however, still unknown to what extent the observed changes are sustainable or whether it is possible to continue to accumulate benefits as a result of long-term adherence. ${ }^{16,18}$ Due to new technological developments, such as pedometers being improved to (tri-axial) accelerometers and mobile phones being transformed into minicomputers, new possibilities for activity monitoring have become available. Numerous activity monitors are commercially available. ${ }^{19}$ For example, the Fitbit provides feedback on steps, distance, and calories. ${ }^{20}$ The activity monitor, Pam, engages the participant by giving points for the activities in a "Pam-score" and detailing a historical overview on a personal website. $^{21}$

Furthermore, systems are developed in which pedometers/accelerometers are connected wirelessly to a mobile phone. ${ }^{22-24}$ This connectedness with mobility makes it possible to give more detailed readily available feedback on a larger screen. Linking selfmonitoring technologies with a coach or embedding such technologies in the care process could further enhance effectiveness of behaviour change strategies, ${ }^{25-27}$ especially when technology and care are carefully developed and aligned with each other. 
In the project "It's LiFe!", an innovative monitoring and feedback tool was developed which is embedded in primary care practice. The tool aims to support the selfmanagement of people with COPD or type-2 diabetes to obtain an active lifestyle by measuring their activity behaviour, giving automatically generated tailored feedback to the patient and to the care professional. The care intervention in which the tool will be embedded is named the Self-management Support Programme (SSP). The programme consists of a limited number of behaviour change consultations with a health care professional.

As a prerequisite for useful technology and a successful intervention that meets the requirements and preferences of end users, it is important to involve the end users in the design process at an early stage. ${ }^{28}$ In the project "It's LiFe!", this inclusiveness of end users was ensured by a user-centred design process in which people with COPD or type2 diabetes and their health care professionals were involved in the development of the technology and the SSP. The aim of this paper is to report on the user-centred design process in which the user requirements for a monitoring and feedback tool were investigated. In particular, users were involved to reveal:

1. Which feedback patients and professionals need to optimally support selfmanagement of physical activity?

2. How this feedback can best be presented?

3. How the tool can be made attractive, persuasive, easy to use and suitable to wear on a daily basis?

\section{Methods}

\section{User-Centred Design process}

A user-centred design (UCD) process was followed. User-centred design is a broad term that describes design processes in which end users influence how a design takes shape. To increase the success rate of the usability in computerized systems, ${ }^{29}$ it is of importance to understand the context of use and the user requirements. 30,31

To ensure UCD from the outset, two patient representatives were recruited from the national patient associations for COPD and diabetes, participated in the research team. These representatives reflected on the needs, demands, and restrictions of the patients. Furthermore, the representatives provided feedback on the comprehensibility of interview questions, the use cases, and other documents which were intended for patient participants in the study. The research team gathered the user requirements and an engineering team translated these into technical solutions. During the development process for the monitoring and feedback tool, there was a continuous interaction between the research team and the engineering team. This interaction facilitated the match between the user requirements and the technical solutions. 
The design process of the monitoring and feedback tool was based on a combination of existing methodologies, but mainly on Shah's methodological framework for medical device development. ${ }^{28,32-34}$ The tool was developed in three iterative stages, depicted as phase A in Figure 1. The end users, people with COPD or type-2 diabetes and their primary care professionals, were extensively involved. In the fourth stage (phase B), the tool will be tested in laboratory situations and real-life settings. The three development stages are described below.

\section{Phase A: Development tool \& SSP}

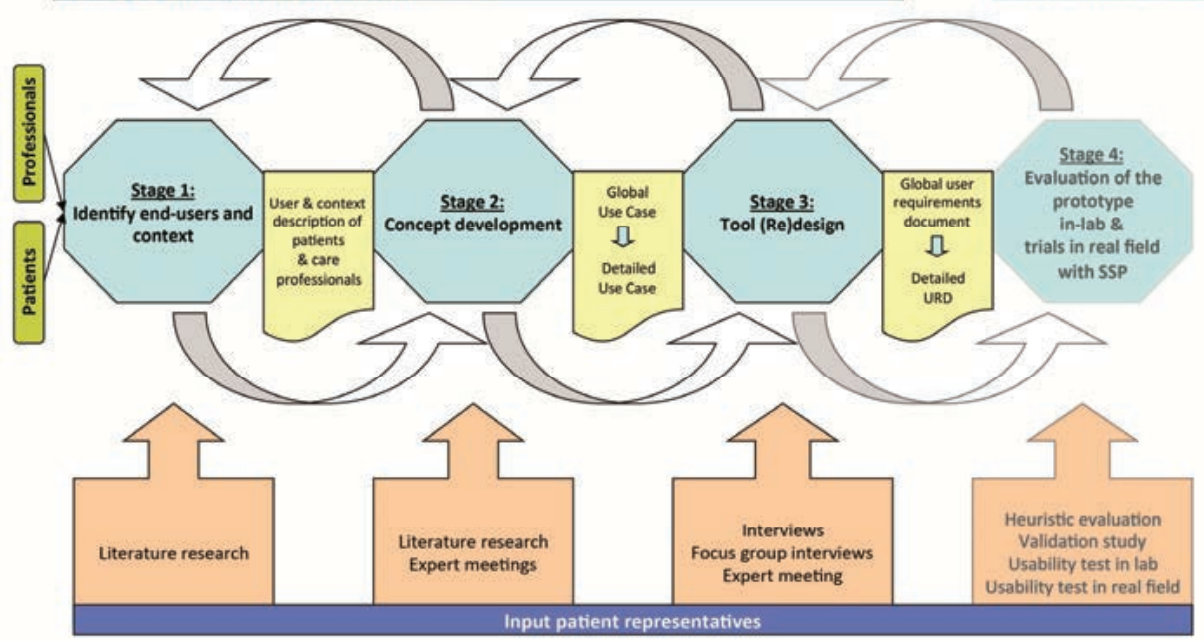

Figure 1 The It's LiFe! user-centred design process. First the end users and context were defined based on the literature. Second the conceptual idea of the tool was described in a use case, based on input from literature, an experts meeting and patient representatives. In stage 3 the use case was discussed with patients and health care professionals to elicit the user requirements for the tool. During the whole process the research team deliberated with the engineering team, to find out what was technically possible. After a detailed user requirements document was composed the engineering team translated the user requirements in technical solutions.

\section{Stage 1: Identify end users and context}

To outline the context in which the monitoring and feedback tool should be used, end users' and environmental characteristics were identified by analysing the literature and clinical practice guidelines. ${ }^{8,9}$ This resulted in a narrative description of users and context.

\section{Stage 2: Concept development}

Literature was studied about behaviour change strategies and technologies to stimulate physical activity that would match with users and context. The user and context description and the literature findings were discussed with experts (physicians, human- 
movement scientists, technicians, and implementation experts). The results of stage 2 were specified in a global use case; describing the interaction between a user and the system to be developed in a step-by-step manner. ${ }^{35}$ The use case was designed to demonstrate the conceptual idea of the tool to end users, without giving too much direction to their thoughts.

\section{Stage 3: Tool (Re-) design}

In order to elicit user requirements for the tool, the use case was the object of discussion with patients in 15 semi-structured interviews (in 2 rounds), and 2 focus group discussions were held ( 1 for COPD and 1 for diabetes. In the focus groups (FG), the patients discussed and complemented the interview results. After another 16 interviews with health care professionals, all of the results were discussed with the same experts from stage 2 plus an independent eHealth researcher and an opinion leader from a general practice. For the interview topics see Table 1. After the interviews and focus group discussions, a first draft of the user requirements document was established. The requirements document was deliberated upon with all of the project members, particularly with the engineering team, to confirm the technical possibilities and to ensure that no important issues were neglected.

Table 1 Interview topics regarding the tool for patients and professionals.

\begin{tabular}{ll}
\hline Main topics & Subtopics \\
\hline Tool architecture & Place activity sensor \\
Roal setting & $\begin{array}{l}\text { Requirements activity sensor } \\
\text { What kind of goal }\end{array}$ \\
Feedback & $\begin{array}{l}\text { On what condition should the goal be adaptable } \\
\text { 1. Amount of activity }\end{array}$ \\
& $\begin{array}{l}\text { In what unit should it be presented } \\
\text { 2. Amount of activity compared to goal }\end{array}$ \\
$\begin{array}{l}\text { In what format should it be visible } \\
\text { In what unit should it be presented } \\
\text { 3. Response of a health professional based on }\end{array}$ & $\begin{array}{l}\text { Where should it be visible } \\
\text { In what format should it be visible } \\
\text { Whe activity results }\end{array}$ \\
\hline
\end{tabular}




\section{Recruitment and data sampling}

Patients and health care professionals were recruited by snowball sampling, by using contacts from the national patient associations and the researchers' networks. Interviews lasted approximately 90 minutes and were held in the respondents' natural environment (at home) or at Maastricht University. Interviewees were asked to read the use case and give their opinion about the conceptual idea of the technology, integration into primary care and their specific requirements for such a tool. During the interviews, the questions and the use case were continuously specified.

\section{Data analysis}

All of the interviews and focus group discussions were audio-taped with the consent of the respondents. The first 8 interviews with patients and all interviews with health care professionals were transcribed verbatim. Transcripts were generated, read, and opencoded using the NVivo 2.0 software package. Two researchers independently opencoded 4 interviews ( 2 from patients and 2 from health care professionals) and reached a consensus about final coding with themes and subthemes. Next, all of the transcripts were (re-)coded using the themes and subthemes as an analytical coding scheme that further evolved during the analysis. After analysis of interviews for round 1 (IR1), questions for each end user group were rephrased from open to closed questions. For example, in the first interview round the patients were asked, "where do you want to wear the activity sensor", whereas in the interviews for round 2 (IR2) all of the previously mentioned answers which were technically possible were given for the patient to choose from. However, other additional options were also welcomed. The audio recordings of the second round of patient interviews and the focus groups were transcribed per code and analysed by two researchers independently. By means of member check, the results of the focus group discussions were verified by a focus group participant and an observer who was present at both of the focus group discussions.

This study was approved by the medical ethical committee of azM/UM.

\section{Results}

\section{Stage 1: User and context description}

Concerning the users and context, it was considered that the resulting intervention (tool + SSP) would be focused on anybody who will benefit from support during physical activity. However, for the scope of the research project, people with COPD or type-2 diabetes, aged less than 70 and over 40 , treated in a primary care setting were chosen. These two patient groups were chosen since they represent a large part of the chronically ill 
people in primary care and can both benefit from lifestyle changes. More importantly, these two groups have diverse physiological and psychological states and therefore different support needs. ${ }^{36}$ Involving this heterogeneous group in the development should lead to a tool that is applicable for a wide audience. However, this implies that the tool should have customizable components to meet the specific needs of different target groups. It was also decided to develop an intervention for people in the contemplation (thinking about change) and preparation (making small changes in behaviour but not enough) phase of the Trans-theoretical Model of Behaviour Change. ${ }^{37,38}$ We believe that people in these stages benefit most from support in self-management. People in the precontemplation phase need to be convinced of the importance of an active lifestyle first. Based on the clinical practice guidelines, ${ }^{8,9}$ the practice nurse was the logical health care professional to be involved.

\section{Stage 2: Concept development}

\section{Literature findings}

In order to develop an effective intervention, it must be clear which determinants are relevant for the target behaviour and which of them can be influenced. For the initiation and maintenance of physical activity, the relevant and changeable personal determinants are: awareness, knowledge, attitude, self-efficacy, intention and intrinsic motivation. $^{39,40}$ Strategies to influence these determinants include: self-monitoring, providing tailored feedback, providing information, action planning, working with role models, and proposing activities that are feasible for the patient. ${ }^{40}$ It is important to note that intention to change is not sufficient; intentions account for only $20-30 \%$ of the variance in behaviour. ${ }^{41}$ In order to narrow the gap between intentions and actual behaviour, it is important to set realistic goals and to identify potential barriers. The Goal-Setting Theory states that a goal should be specific, challenging but realistic, set by the patient himself (or in collaboration with the health care professional), and easily monitored. ${ }^{42}$

Physical activity can be measured with questionnaires, energy expenditure measurements and activity monitors. For daily use, activity monitors are most suitable. ${ }^{43}$ There are three classes of activity monitors; pedometers, accelerometers and integrated multisensory systems. Pedometers estimate the number of steps taken but are limited to measurement of the vertical plane. Accelerometers detect acceleration in one or three directions and can determine the amount, intensity and duration of movements. Integrated multisensory systems try to optimize physical activity assessment using the combination of accelerometry and other sensors that measure physiological responses to exercise, such as skin temperature or heart rate. However, there is little evidence that adding another physiological measure significantly improves the assessment of energy expenditure. ${ }^{44}$ Numerous accelerometers are developed with different wearing positions, such as the hip, waist, ankle, upper leg, and wrist. An accelerometer is most accurate in assessing daily life physical activity if worn on the lower back or hip. ${ }^{44}$ However at 
this position, cycling is not captured very well. A promising development is monitors that integrate Global Positioning Systems ${ }^{43}$ which could make it possible to measure cycling. Unfortunately at the moment, this is too energy consuming for daily use.

From other monitoring and feedback tools it was learnt that feedback and incentives should be provided whenever progress is made and not only when the goal is achieved. ${ }^{18}$ From the development and evaluation of two mobile systems, Houston ${ }^{23}$ and Ubifit ${ }^{24}$, it can be seen that mobile interventions can be a powerful way of promoting health behaviour changes. This is achieved by supporting the persistent activation of health goals, focusing on patterns of activity, and facilitating optional social support. ${ }^{45}$

\section{Global Use Case}

The literature findings and meetings with experts led to the following concept of the tool. This concept of the tool was elaborated in the global use case, which was presented as a narrative scenario, and was the input for stage 3. Basically, the tool, consisting of a sensor and a feedback system, will focus on the stimulation of daily activity and not on sports. The sensor is placed somewhere on the body and measures physical activity. The patient sets a personal activity goal together with the health care professional, and receives feedback about the current activity level related to the pre-set activity goal. The health care professional and a relative also receive a periodic summary of the activities. When the patient is performing well, the patient receives compliments from the tool, the health care professional, and their relative.

\section{Stage 3: Tool (Re-) design}

The purpose of this stage was to further specify the conceptual idea to the user requirements and preferences of the patients and health care professionals. The characteristics of patients and professionals who participated in the interviews and focus groups are described in Tables 2. Four main topics relevant for the tool were identified from the interviews: (1) Tool architecture; (2) Goal setting; (3) Feedback; and (4) Data sharing. For each topic, user requirements and preferences were elicited. For the resulting design of the tool and a visualization of the feedback loops (described under "feedback consequences") see Figure 2. 
Table 2 Characteristics of respondents of the interviews and focus groups.

\begin{tabular}{llll}
\hline People with COPD & $\begin{array}{l}\text { Interview round 1 } \\
(n=4)\end{array}$ & $\begin{array}{l}\text { Interview round 2 } \\
(n=3)\end{array}$ & $\begin{array}{l}\text { Focus group } \\
(n=6)\end{array}$ \\
\hline Mean age (SD) & $64(7.2)$ & $61.5(5.3)$ & $61.8(5.7)$ \\
GOLD & $2-4$ & $3-4$ & $2-4$ \\
\hline People with DM 2 & $\begin{array}{l}\text { Interview round 1 } \\
(n=4)\end{array}$ & $\begin{array}{l}\text { Interview round 2 } \\
(n=4)\end{array}$ & $\begin{array}{l}\text { Focus group } \\
(n=5)\end{array}$ \\
\hline Mean age (SD) & $61.5(5.3)$ & $62.8(12.8)$ & $56.8(8.2)$ \\
\hline Healthcare professionals & Interview round 1 & Interview round 2 & $(n=5)$ \\
& $(n=11)$ & 5 & \\
\hline Practice Nurse & 2 & 0 & \\
Diabetes Nurse & 2 & 0 & \\
Pulmonary Nurse & 2 & 0 & \\
General Practitioner & 3 & 0 & \\
Physiotherapist & 2 & 42 & \\
Mean age & 42 & & \\
\hline
\end{tabular}

\section{Activity sensor}

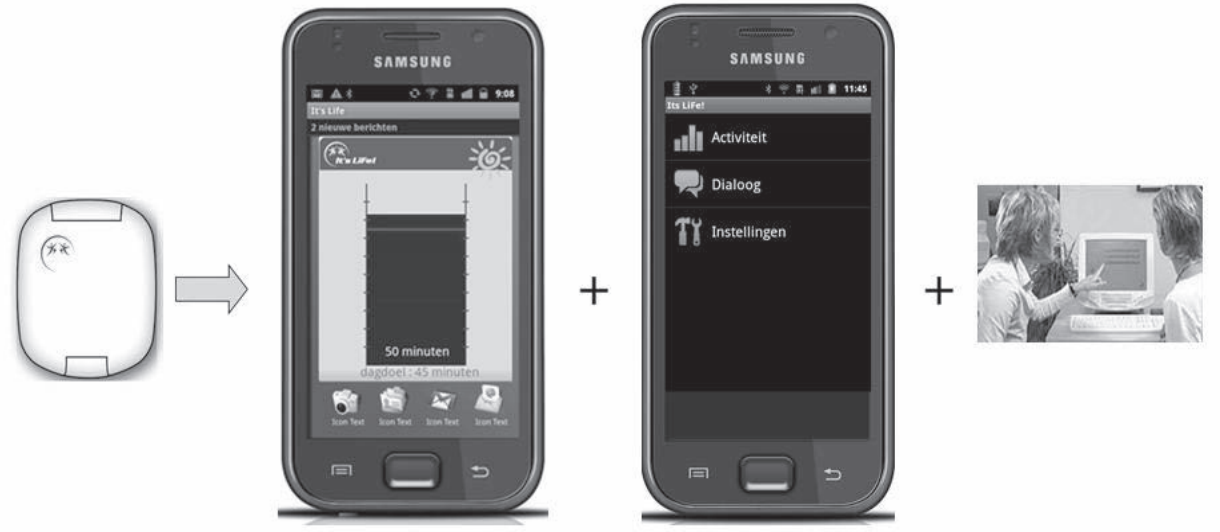

\section{Feedback 1}

Real time compared with activity day goal

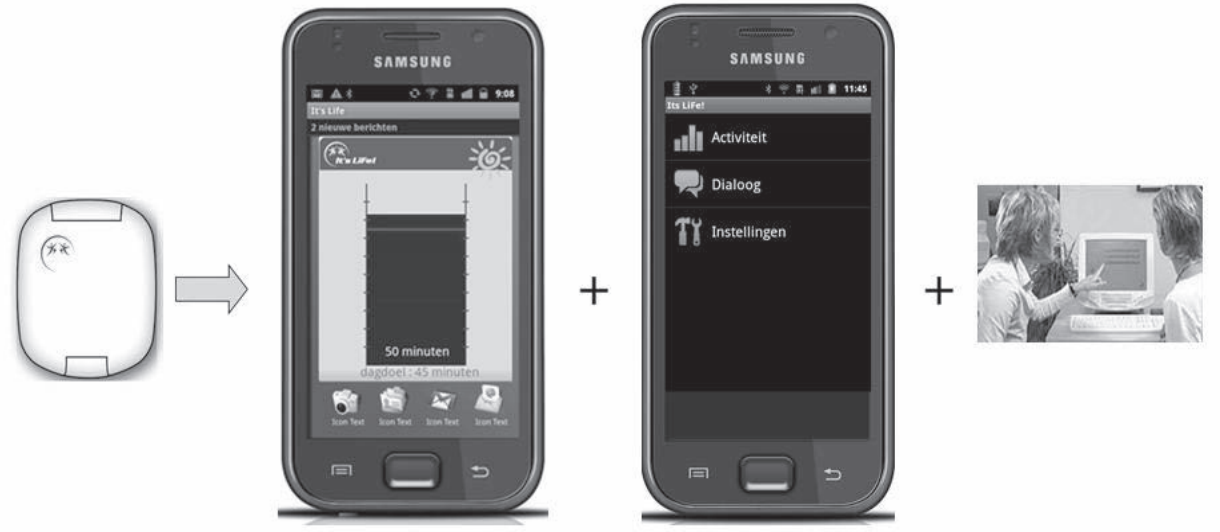

\section{Feedback 2}

Dialogue sessions and feedback messages on app and website

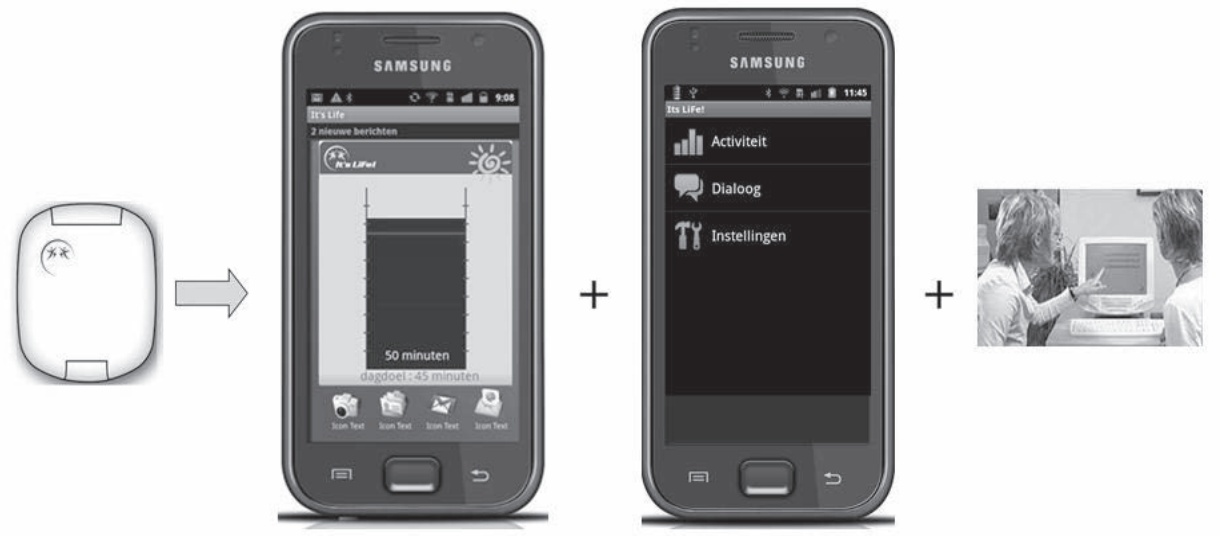

\section{Feedback 3}

Practice nurse reacts on results based on results on web client

Figure 2 The monitoring and feedback tool that was developed, based on the requirements of the end users. The tri-axial activity sensor is connected via Bluetooth to the smartphone. The smartphone gives directly visible feedback about the amount of activity in a bar chart, which dynamically fills up. When the goal (indicated by a red line) has been reached, a sun rises. In the app and on a secure webpage, people can see their activity history and answer dialogue sessions and read feedback messages generated by the system. The practice nurse can monitor the results of all patients on the secure web page to discuss during patient visits.

\section{Tool architecture}

During the interviews, several requirements relevant for the activity sensor arose. The most important requirements were that it should measure all activities. The specific 
design was not important as long as it did not hinder movements or was obtrusive. For the location of the sensor, the hip and wrist were the most popular.

A place where it is not visible and does not hinder you. If you wear a wristwatch for example, people may ask you what it is. That is nice for the first few times, but after 13 times it is not. So something like a watch, but then for around your ankle. Or something around the belt that is always hidden. [IR2, DM2, man, 60 years]

Only two respondents did not prefer a sensor on the hip, with the argument that it may be problematic for women wearing a dress and people doing many arm activities.

I think this is a man's idea, since a woman cannot wear this sensor when they wear a dress. And people with COPD GOLD 1 or 2 do not feel sick yet, so they will not wear an inelegant device. [FG, COPD, female, 57 years]

Immediate feedback should be visible at a glance. Respondents preferred to receive feedback on a mobile phone, since it has a larger screen than an activity sensor and is more readily accessible than a computer. For more comprehensive use, however, such as manual data entry and consulting activity histories, a computer was considered more feasible.

\section{Consequences for tool architecture}

Based on the results above, it was decided that the "It's LiFe!" tool would consist of three elements (presented in Figure 2): 1. An activity sensor with Bluetooth connectivity worn on the hip and clipped on the belt. 2. A smartphone with an app for mobile feedback. 3. A Web client for comprehensive feedback and data entry for patient and practice nurse. The "It's LiFe!" activity sensor is a 3D accelerometer with a sample frequency of $25 \mathrm{~Hz}$. This newly developed activity sensor ( $4.0 \times 4.5$ centimetre) is based on the Ciro Activity Monitor (CAM) also manufactured by Maastricht Instruments. ${ }^{46}$ The validity of the sensor will be tested in a subsequent study on a treadmill and in free-living conditions. The smartphone and web client would be connected to a web server with facilities for data storage and feedback generation. Nearly all of the user requirements were met. For budget reasons, however, the activity sensor could not be made suitable for cycling and swimming. It was decided that the amount of swimming and cycling activities should be entered by the users manually. The final architecture of the activity sensor was chosen from two prototypes by the patient representatives.

\section{Goal setting}

Patient respondents preferred to set their goals together with the practice nurse. This would prevent the under- or overestimation of their abilities. In general, daily goals were 
preferred since weekly goals lead to postponing activities. Some respondents with COPD, however, indicated that they feel different every day, so they did not like the idea of a static goal per day. Patients indicated that they preferred to be able to change the goal themselves because they do not see the health care professional often enough. However, changing goals should only be possible after a permissive message from the tool, to prevent downwards adjustments too easily.

\section{Consequences for goal setting}

Based on the results above, it was decided to set goals in a three-step process.

1. Calibration. During a two-week pre-measurement, the activity pattern of the users will be assessed, in order to set a realistic goal that is based on an objective measurement. In addition, the patient will receive questions (dialogue sessions) on the smartphone and website to identify which kind of activities the patient prefers and which barriers have to be overcome.

2. Goal setting. After the initial two weeks, the results of the pre-measurement will be evaluated by the practice nurse and discussed with the patient. Together, they will set an appropriate goal in minutes of moderate to vigorous physical activity per day. Appropriate means challenging, but within realistic margins, and personalized. Patients can adapt their goal themselves after a permissive message based on their activity results or by contacting the practice nurse.

3. Activity planning. Once at home, the patient will be invited by the tool to plan concrete daily activities to reach their goal. This will be facilitated by a dialogue session on the smartphone or on the website (at choice). This trigger to plan activities in detail (such as when, with whom and where you will be active) will narrow the gap between intention and behaviour and make it more likely that the patient will reach the goal. ${ }^{47}$

\section{Feedback}

There was consensus among respondents about how daily physical activity should be visualized on the smartphone. Activity performance should be set out against daily goals at any time. Performance data should be formatted as minutes of activity per day, rather than in calories (too complicated) or points (too abstract). Performance should be denoted in percentages of their goal, visualized in images, and colour, but not using a childish animation. People did not state a preference to hear a sound when they reach their goal because it could interrupt them, could be noticed by others, and may become irritating. To monitor progress or decline, a historic overview of activity results over the last few months was deemed important. Some respondents indicated that they wanted to distinguish intensity rather than the type of activity (i.e., sitting, biking, or swimming). Some respondents wanted to see a difference between moderately-intense and highintensity activities, because this would stimulate them to do more high-intensity activi- 
ties and they also wanted to receive more credits for those activities. Other respondents argued that they will feel the intensity themselves and that they will be happy to meet their goal anyway. This led to a lot of discussion during the focus groups and in the technical and research meetings. Another point of discussion was the choice between an absolute or relative threshold between the levels of intensity. Advocates of a relative threshold stated that everybody would experience high-intensity activities differently; for some chronically ill patients, a walking pace of $3 \mathrm{~km}$ per hour is exhausting, for others $5 \mathrm{~km}$ per hour is more appropriate. A relative threshold could be set per individual, based on a two week pre-measurement period. However, respondents with COPD also indicated that the difficulty of being active may differ from day to day.

Even for me, as an individual person, it is very hard to set a threshold. One day I am very fit, I exercise and nothing is wrong, the next day the ambulance is needed! [FG, COPD, male, 65 years]

Everybody agreed that details about intensity need to be visible at a glance. Feedback messages from the server must be short, subtle, and positive in nature; without being paternalistic. Most respondents liked the idea that a health care professional could monitor their activity performance, because this would be an additional motivational factor. However, people thought they should also be able to make annotations to the activity data, in order to explain lower performance if one was sick (dyspnea), the weather was bad, etc.

Yeah, I think if a health care professional can watch your results it has a psychological effect. You don't want to disappoint the people who pay attention to you. [IR 1, DM2, female, 62 years]

\section{Consequences for feedback}

Based on the results above, it was decided that feedback will be given in 3 loops. 1. In the first loop, data is directly visible on a widget on the smartphone. In a bar diagram, realized activity in minutes ( $\geq 3$ METS) is compared with the daily goal (see Figure 2 ). When the daily goal has been reached, a sun becomes visible as a subtle reward (see Figure 2). In addition, when opening the app, historic activity data can be viewed; in minutes per day, aggregated in days, weeks or months, as well as distinguishing between moderate (3-5.9 METS) and intense ( $\geq 6$ METS) activities (see Figure 3). 2. In the second loop, periodic feedback messages will be sent after 3, 5, or 14 days. Various messages will be used, depending on the progress of performance, such as encouragement, positive trends, rewards, and suggestions to overcome barriers or to adjust personal goals. 3 . In the third loop, users will receive feedback from the practice nurse. This will happen after 2 to 3 months and after 6 months, when the patient visits the practice nurse to evaluate the results and discuss barriers and facilitators. In between consultations, the 
practice nurse can monitor the activity results and is free to choose whether to react to this or not.

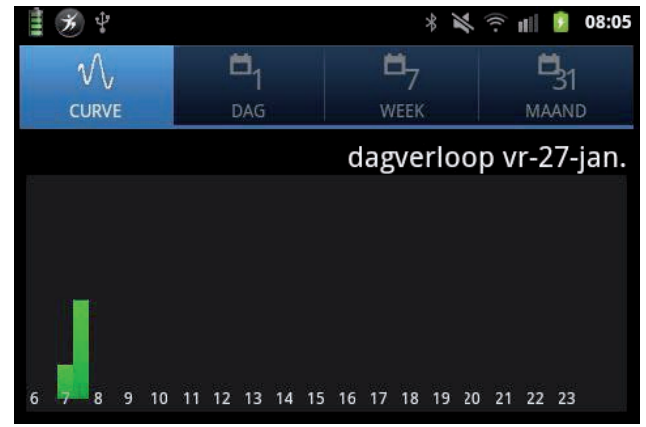

A. Day view per hour from 6am to $11 \mathrm{pm}$.

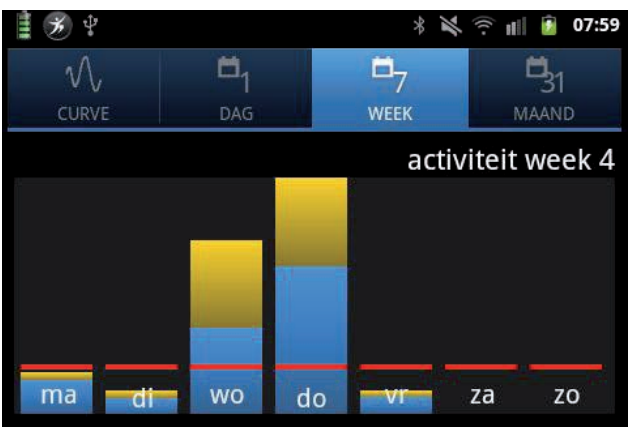

C. Week view.

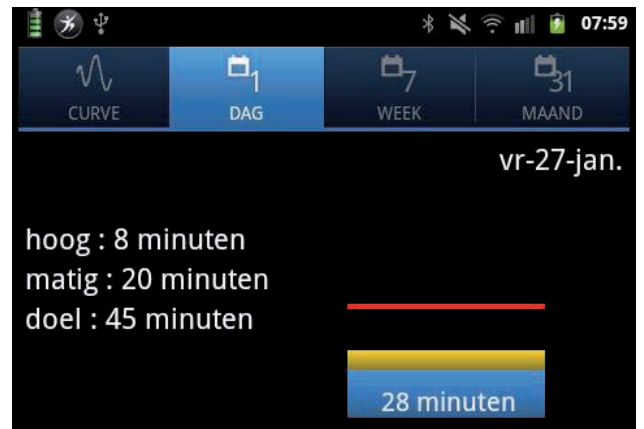

B. Day view. 'Hoog' is 'high'. 'matig' is 'moderate', 'doel' is 'goal', 'minuten' is 'minutes'.

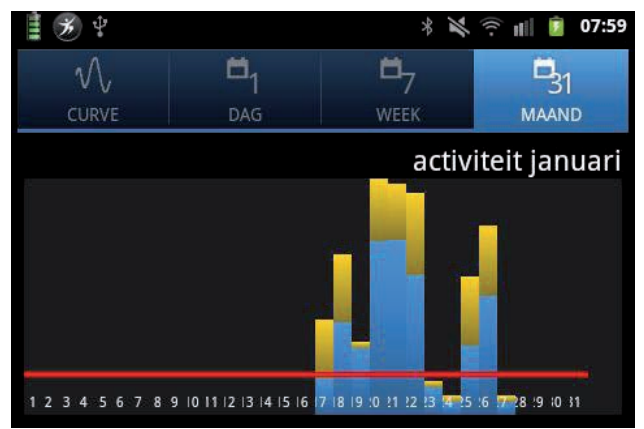

D. Month view.

Figure 3 Activity menu on the smartphone app. The blue part of the bars indicates the moderately intense activity in minutes; the yellow part denotes the high-intensity activities. The red line indicates the daily goal.

\section{Data sharing}

Although most respondents perceived peer support as being important, only a few respondents were willing to share their activity results with relatives or peers. (e.g., on a forum or social network, such as Facebook or Twitter.)

Sharing results on the internet is not motivating because the data and people are anonymous. Mutual support in real life is. And then you can decide to go hiking together. [IR2, male, DM2, 60 years] 


\section{Consequences of data sharing}

Activity data will only be shared with the practice nurse and not with peers. Sharing data through social media is not a priority. However, the involvement of relatives in the process of becoming more active will be encouraged in other ways.

\section{Requirements of health care professionals}

Health care professionals admitted that they usually pay too little attention (approximately $10 \%$ of the consultation-time) to physical activity and that they welcome technological support to improve this. Most professionals viewed the tool as a mainly diagnostic instrument, since patients generally overestimate their level of physical activity. The activity pattern should be presented to them in time, intensity, steps, METS or calories, with aggregated information about the patients' adherence and goal attainment. For their own convenience, they preferred to consult activity data within their own patient information system.

Regarding goal setting, professionals agreed on setting a goal together with the patient. Goals should be flexible, personal, and comorbidities should be taken into account. There was little to no enthusiasm, however, about the idea of giving feedback to the patients themselves in between consultations. An alternative idea of automated feedback messages was more appreciated. Professionals agreed with the patients that feedback messages should be positive (with a smiley face or flower), short and clear, but not pedantic. Performance should be visualized in numbers and graphs, including visible trends.

\section{Design principles concerning the requirements of health care professionals}

Based on the information above, it was decided that practice nurses will have their own web client (The "It's LiFe!" Monitor) with two levels of information: 1. An overview window with aggregated information about the status of their patients' goals. 2. A detail window presenting activity minutes per day and results from dialogue sessions. With this information, the practice nurse can better prepare the patient consultation and can estimate appropriate patients' goals more readily. The total physical activity counselling protocol will be published elsewhere. The integration with different patient information systems will not be realized during the "It's LiFe!" project. 


\section{Discussion}

\section{Principal results}

In this study, the UCD process of a monitoring and feedback tool to enhance the selfmanagement of physical activity for patients with COPD or type-2 diabetes is described. The research team gathered the user requirements and an engineering team translated these into technical solutions, to avoid data gathering with presuppositions. This tool is designed to be combined with a Self-management Support Programme for embedding in primary care. It provides a combination of behaviour change techniques to increase knowledge, awareness (by self-monitoring) and self-efficacy. Personal goals are set and personalized feedback is provided based on the degree of goal attainment. The usercentred design process gave insight into the wishes and needs of the end users, which will increase the likelihood of success. The main requirements for the tool derived from this process were:

- An activity sensor placed on the hip that measures activity accurately.

- Goals set in collaboration with the practice nurse after a pre-measurement period, in minutes activity per day. Personalized goals tailor the tool to individual needs.

- Feedback provided at different levels: immediate feedback, visible on the smartphone as a percentage of their daily activity goal, or presented as an image and in colour; periodic feedback messages, always given with positive verbal; and aggregated feedback to the practice nurse, which should be used during the patient consultations.

- Activity data sharing with a care professional, not with peers on a forum or social media.

- An opportunity, for the patient to make annotations to their activity pattern.

Both end user groups did not agree on all requirements. Patients want support from the practice nurse in between consultations, while care providers indicated that this is unmanageable due to time constraints. Therefore, automated feedback was incorporated to fulfil the need of patients of extra support. This makes it more suitable for daily practice in primary care. Most respondents were not open for sharing activity data on social media this may be influenced by the age of this group. The monitoring and feedback tool should be prepared for changes in this attitude.

\section{Limitations}

A convenient sample of people with COPD or type-2 diabetes and health care professionals was used. Those who were interested in issues related to physical activity and/or technology may have been more likely to participate compared with others. Consequently, the study may have a self-selection bias. On the other hand, credibility $^{48}$ was increased by involving patient representatives in the research team in all decisions, and 
by the use of multiple data collection methods. Reliability was ensured by investigator triangulation, since the interviews were held by two different researchers and multiple researchers were involved in the analysis and the interpretation of data.

Other limitations included contextual restraints, such as budget, time, and the capabilities of technology in general and the engineering team. This led to some concessions, such as an activity sensor which is not waterproof, which mean there could be no registration of activity during swimming. Furthermore an activity sensor worn on the hip, is not able to register cycling. This may cause frustration among users that spend considerable time on these activities. It may also discourage users from developing these activities. The possibility to make annotations should compensate for this limitation.

\section{Comparison with prior work}

Prior work has documented the effectiveness of pedometers to increase physical activity. ${ }^{16-18}$ However, these were all short-term studies. It is unknown to what extent these changes are sustainable, since pedometers are still not routinely used in health care. An exceptional feature of our tool is the automated connection to the primary care professional via a secure website. Furthermore, the tool is embedded in a support programme which is carefully aligned and simultaneously developed with the tool. Patients in this study indicated that the combination with coaching from the health care professional is a benefit, since health care professionals can serve as an extra motivator.

In this study, we developed a monitoring and feedback system in an iterative process inclusive of patients and health care professionals, to enhance the likelihood of success. While this study was conducted, a framework was published to improve the uptake and impact of eHealth technologies. ${ }^{49}$ This framework, which is based on an extensive review, confirms the importance of end users' participation and an iterative development process. Furthermore, this framework emphasizes the importance of taking the conditions for implementation into account during the development process. In this study, this was achieved by involving health care professionals in the project and also by developing a Self-management Support Programme for the tool, which describes how the health care professional can support the patient.

The final design of the monitoring and feedback tool is in agreement with the proposed design strategies from Consolvo for technologies that support behaviour change. $^{50}$ These strategies are based on the experiences from three persuasive technology interventions: Breakaway, ${ }^{51}$ Fish ' $n$ ' Steps ${ }^{52}$ and Houston. ${ }^{23}$ Consolvo's 8 proposed strategies are: (1) Abstract \& Reflective, (2) Unobtrusive, (3) Public, (4) Aesthetic, (5) Positive, (6) Controllable, (7) Trending/Historical, and (8) Comprehensive.

In addition, the developed tool, together with the Self-management Support Programme, is in line with Fogg's theory of persuasive technology. According to Fogg, an intervention to change people's behaviour should focus on ability and motivation and provide a trigger to change. ${ }^{53}$ Our intervention targets people who have the motivation 
to change their behaviour but have not previously managed to do so. The selfmonitoring tool makes people aware of their inactivity, which can lead to further motivation. The patient's abilities are taken into account in the dialogue sessions, personal goals, and support from the practice nurse. In addition, our intervention provides a trigger to act by delivering feedback on physical activity on a timely basis and in an actionable format, namely related to tangible personal goals.

\section{Conclusions}

In this paper, the development process of a monitoring and feedback tool is described as the preparation of an intervention to support the self-management of physical activity. It illustrates how a user-centred approach allows the consideration of valuable details to make the fit between the user, technology, and organization of care, which is important for the usability and acceptability of the tool. The leading principle of this intervention is to change behaviour by self-monitoring, goal-setting, and feedback. The tool connects three technologies: an accelerometer, a smartphone app, and an Internet application. Feedback is given in three loops: direct feedback on daily activity compared with personal targets, periodic feedback on historical performance, and personal feedback by the practice nurse during consultations.

Having followed a user-centred design, we expect that the usability and acceptability of the tool has increased. This will be tested in a usability study in a lab environment and a pilot study in two general practices. The effect of the final tool embedded in primary care will be evaluated in a cluster randomized controlled trial.

\section{Acknowledgments}

This study was funded by the Netherlands Organization for Health Research and Development (ZonMw). Publication of the manuscript was supported by NWO, the Netherlands Organization for Scientific Research. We would like to thank the respondents and health care professionals who were willing to share their time, thoughts and experience with us and especially the patient representatives, Jos Donkers and Ina van Opstal, for their critical remarks. The companies involved in the development are Maastricht Instruments BV, Oxfordlaan 70, 6229 EV Maastricht, the Netherlands, IDEE Maastricht UMC+ Universiteitssingel 50, 6229 ER Maastricht, the Netherlands and Sananet Care BV, Rijksweg Zuid 22A, 6131 AP Sittard, the Netherlands. 


\section{References}

1. Organization WH. Global Recommendations on Physical Activity for Health. 2011;

http://www.who.int/dietphysicalactivity/physical-activity-recommendations-18-64years.pdf?ua=1 Archived at: http://www.webcitation.org/6PF1UBuEa. Accessed May 1 2014, 2014.

2. Guthold R, Ono T, Strong KL, Chatterji S, Morabia A. Worldwide variability in physical inactivity a 51country survey. Am J Prev Med. Jun 2008;34(6):486-494.

3. Bauman A, Bull F, Chey T, et al. The International Prevalence Study on Physical Activity: results from 20 countries. Int J Behav Nutr Phys Act. 2009;6(1):21.

4. Langer D, Hendriks E, Burtin C, et al. A clinical practice guideline for physiotherapists treating patients with chronic obstructive pulmonary disease based on a systematic review of available evidence. Clin Rehabil. May 2009;23(5):445-462.

5. Stewart AL, Hays RD, Wells KB, Rogers WH, Spritzer KL, Greenfield S. Long-term functioning and well-being outcomes associated with physical activity and exercise in patients with chronic conditions in the Medical Outcomes Study. J Clin Epidemiol. Jul 1994;47(7):719-730.

6. Bourbeau J. Making pulmonary rehabilitation a success in COPD. Swiss Med Wkly. 2010;140:w13067.

7. Miller YD, Dunstan DW. The effectiveness of physical activity interventions for the treatment of overweight and obesity and type 2 diabetes. J Sci Med Sport. Apr 2004;7(1 Suppl):52-59.

8. Netherlands LAt. Care standard COPD Amersfoort. 2010.

9. Federation DD. NDF Standard of care: Transparency and quality of diabetes care for people with type 2 diabetes. NDF Zorgstandaard: transparantie en kwaliteit van diabeteszorg voor mensen met diabetes type 2. Amersfoort: Dutch Diabetes Federation, Nederlandse Diabetes Federatie; 2007.

10. Ursum J, Rijken M, Heijmans M, Cardol M, Schellevis F. Overzichtstudies: Zorg voor chronisch zieken Organisatie van zorg, zelfmanagement, zelfredzaamheid en participatie. 2011.

11. van Achterberg T, Huisman-de Waal GG, Ketelaar NA, Oostendorp RA, Jacobs JE, Wollersheim HC. How to promote healthy behaviours in patients? An overview of evidence for behaviour change techniques. Health Promot Int. Jun 2011;26(2):148-162.

12. Shilts MK, Horowitz M, Townsend MS. Goal setting as a strategy for dietary and physical activity behavior change: a review of the literature. Am J Health Promot. Nov-Dec 2004;19(2):81-93.

13. de Vries $\mathrm{H}$, Kremers SP, Smeets T, Brug J, Eijmael K. The effectiveness of tailored feedback and action plans in an intervention addressing multiple health behaviors. Am J Health Promot. Jul-Aug 2008;22(6):417-425.

14. Fujii $\mathrm{H}$, Nakade M, Haruyama $\mathrm{Y}$, et al. Evaluation of a computer-tailored lifestyle modification support tool for employees in Japan. Ind Health. Jul 2009;47(3):333-341.

15. Annesi JJ. Effects of computer feedback on adherence to exercise. Percept Mot Skills. Oct 1998;87(2):723730.

16. Bravata DM, Smith-Spangler C, Sundaram V, et al. Using pedometers to increase physical activity and improve health: a systematic review. Jama. Nov 21 2007;298(19):2296-2304.

17. Lindberg R. Active living: on the road with the 10,000 Steps program. Journal of the American Dietetic Association. Aug 2000;100(8):878-879.

18. Tudor-Locke $C$, Lutes $L$. Why do pedometers work?: a reflection upon the factors related to successfully increasing physical activity. Sports Med. 2009;39(12):981-993.

19. Yang CC, Hsu YL. A review of accelerometry-based wearable motion detectors for physical activity monitoring. Sensors (Basel). 2010;10(8):7772-7788.

20. Fitbit. http://www.fitbit.com/one. Archived at: http://www.webcitation.org/6CoJDs9m0 Accessed 14 august 2012, 2012.

21. Damen E. PAM. Welkom bij Pam 2014; http://www.pam.com/index.php?pid=1 . Archived at: http://www.webcitation.org/6PEz8ql7Y. Accessed 1 May 2014, 2014.

22. Arsand E, Olsen OA, Varmedal R, Mortensen W, Hartvigsen G. A system for monitoring physical activity data among people with type 2 diabetes. Studies in health technology and informatics. 2008;136:113-118. 
23. Consolvo S, Everitt K, Smith I, Landay JA. Design requirements for technologies that encourage physical activity2006.

24. Consolvo S, McDonald DW, Toscos T, et al. Activity sensing in the wild: a field trial of ubifit garden2008.

25. Leemrijse CJ, van Dijk L, Jorstad HT, Peters RJ, Veenhof C. The effects of Hartcoach, a life style intervention provided by telephone on the reduction of coronary risk factors: a randomised trial. BMC cardiovascular disorders. 2012;12:47.

26. Appel LJ, Clark JM, Yeh HC, et al. Comparative effectiveness of weight-loss interventions in clinical practice. N Engl J Med. Nov 24 2011;365(21):1959-1968.

27. Varnfield M, Karunanithi MK, Sarela A, et al. Uptake of a technology-assisted home-care cardiac rehabilitation program. Med J Aust. Feb 21 2011;194(4):S15-19.

28. Shah SG, Robinson I, AlShawi S. Developing medical device technologies from users' perspectives: a theoretical framework for involving users in the development process. Int J Technol Assess Health Care. Oct 2009;25(4):514-521.

29. Abras C, Maloney-Krichmar D, Preece J. User-centered design. Thousand Oaks: Sage; 2004.

30. ISO ISO. 13407: Human-centred design processes for interactive systems. Geneva: ISO1999.

31. ISO. ISO 9241-210:2010. Ergonomics of human-system interaction -- Part 210: Human-centred design for interactive systems 2010 .

32. De Rouck S, Jacobs A, Leys M. A methodology for shifting the focus of e-health support design onto user needs:A case in the homecare field. INTERNATIONAL JOURNAL OF MEDICAL INFORMATICS. 2008;77(9):589-601.

33. Poulson D, Ashby M, Richardson S. Userfit : a practical handbook on user-centred design for Assistive Technology. Brussels: ECSC-EC-EAEC; 1996.

34. MATCH MAOTCFH. User requirements during the product development pathway. 2010; http://matchguide.brunel.ac.uk/glossary/UCD.aspx . Archived at: http://www.webcitation.org/6CoFmB25e, 2010.

35. Jacobson I. Object-oriented software engineering : a use case driven approach. [New York]; Wokingham, Eng.; Reading, Mass.: ACM Press ; Addison-Wesley Pub.; 1992.

36. Corcoran KJ, Jowsey T, Leeder SR. One size does not fit all: the different experiences of those with chronic heart failure, type 2 diabetes and chronic obstructive pulmonary disease. Australian Health Review. 2013;37(1):19-25.

37. Prochaska JO, DiClemente CC. Stages and processes of self-change of smoking: toward an integrative model of change. J Consult Clin Psychol. 1983;51(3):390.

38. Marshall SJ, Biddle SJH. The transtheoretical model of behavior change: a meta-analysis of applications to physical activity and exercise. Annals of Behavioral Medicine. 2001;23(4):229-246.

39. Fishbein M, Ajzen I. Predicting and changing behavior: The reasoned action approach. New York: Psychology Press (Taylor \& Francis); 2010.

40. van Stralen MM, Kok G, de Vries H, Mudde AN, Bolman C, Lechner L. The Active plus protocol: systematic development of two theory- and evidence-based tailored physical activity interventions for the overfifties. BMC Public Health. 2008;8:399.

41. Gollwitzer PM. Implementation Intentions_Strong Effects of Simple Plans. American Psychologist by the American Psychological Association. 1999;54(7):493-503.

42. Latham GP, Locke EA. Self-regulation through goal setting. Organizational Behavior and Human Decision Processes. 1991;50(2):212-247.

43. Van Remoortel H, Giavedoni S, Raste Y, et al. Validity of activity monitors in health and chronic disease: a systematic review. International Journal of Behavioral Nutrition and Physical Activity. 2012;9(1):84.

44. Plasqui G, Bonomi A, Westerterp K. Daily physical activity assessment with accelerometers: new insights and validation studies. Obesity Reviews. 2013.

45. Klasnja P, Consolvo S, McDonald DW, Landay JA, Pratt W. Using mobile \& personal sensing technologies to support health behavior change in everyday life: lessons learned. AMIA Annu Symp Proc. 2009;2009:338342. 
46. Annegarn J, Spruit MA, Uszko-Lencer NH, et al. Objective physical activity assessment in patients with chronic organ failure: a validation study of a new single-unit activity monitor. Archives of physical medicine and rehabilitation. 2011;92(11):1852-1857. e1851.

47. Sniehotta FF, Scholz U, Schwarzer R. Bridging the intention-behaviour gap: Planning, self-efficacy, and action control in the adoption and maintenance of physical exercise. Psychol Health. 2005/04/01 2005;20(2):143-160.

48. Polit DF, Beck CT. Resource manual to accompany Nursing research: generating and assessing evidence for nursing practice, 8th edition. Philadelphia: Wolters Kluwer Health/lippincott Williams \& Wilkins; 2008.

49. van Gemert-Pijnen JE, Nijland N, van Limburg M, et al. A holistic framework to improve the uptake and impact of eHealth technologies. J Med Internet Res. 2011;13(4):e111.

50. Consolvo S, McDonald DW, Landay JA. Theory-driven design strategies for technologies that support behavior change in everyday life. Proceedings of the 27th international conference on Human factors in computing systems. Boston, MA, USA: ACM; 2009:405-414.

51. Jafarinaimi N, Forlizzi J, Hurst A, Zimmerman J. Breakaway: an ambient display designed to change human behavior2005.

52. Lin J, Mamykina L, Lindtner S, Delajoux G, Strub H. Fish'n'steps: Encouraging physical activity with an interactive computer game. UbiComp 2006: Ubiquitous Computing. 2006:261-278.

53. Fogg B. A behavior model for persuasive design. Proceedings of the 4th International Conference on Persuasive Technology. Claremont, California: ACM; 2009:1-7. 



\section{CHAPTER 3}

\section{Usability testing of a monitoring and feedback tool to stimulate physical activity}

This chapter was published as:

van der Weegen S, Verwey R, Tange HJ, Spreeuwenberg MD, de Witte LP. Usability testing of a monitoring and feedback tool to stimulate physical activity. Patient Prefer Adherence. 2014;8:311-322 


\section{Abstract}

Introduction A monitoring and feedback tool to stimulate physical activity, consisting of an activity sensor, smartphone application (app), and website for patients and their practice nurses, has been developed: the 'It's LiFe!' tool. In this study the usability of the tool was evaluated by technology experts and end users (people with chronic obstructive pulmonary disease or type 2 diabetes, with ages from 40-70 years), to improve the user interfaces and content of the tool.

Patients and methods The study had four phases: 1 ) a heuristic evaluation with six technology experts; 2) a usability test in a laboratory by five patients; 3 ) a pilot in real life wherein 20 patients used the tool for 3 months; and 4) a final lab test by five patients. In both lab tests (phases 2 and 4) qualitative data were collected through a thinking-aloud procedure and video recordings, and quantitative data through questions about task complexity, text comprehensiveness, and readability. In addition, the post-study system usability questionnaire (PSSUQ) was completed for the app and the website. In the pilot test (phase 3), all patients were interviewed three times and the Software Usability Measurement Inventory (SUMI) was completed.

Results After each phase, improvements were made, mainly to the layout and text. The main improvement was a refresh button for active data synchronization between activity sensor, app, and server, implemented after connectivity problems in the pilot test. The mean score on the PSSUQ for the website improved from 5.6 (standard deviation [SD] 1.3) to 6.5 (SD 0.5), and for the app from 5.4 (SD 1.5) to 6.2 (SD 1.1). Satisfaction in the pilot was not very high according to the SUMI.

Discussion The use of laboratory versus real-life tests and expert-based versus userbased tests revealed a wide range of usability issues. The usability of the It's LiFe! tool improved considerably during the study. 


\section{Introduction}

Increased physical activity is associated with improvements in many health conditions, including cardiovascular diseases, obesity, insulin insensitivity, osteoporosis, and psychological conditions. ${ }^{1,2}$ Therefore, guidelines recommend taking moderately intense aerobic physical activity for a minimum of 30 minutes on 5 days each week or vigorousintensity aerobic activity for a minimum of 20 minutes on 3 days each week, in order to maintain health. ${ }^{3,4}$ However, many people do not meet these criteria, with percentages ranging from $41 \%$ in the Netherlands to $66 \%$ and $53 \%$ in the UK and USA. ${ }^{4-7}$ It seems difficult to be sufficiently active, especially for people with a chronic disease. ${ }^{8,9}$ In a Dutch sample, $66 \%$ of the people with chronic obstructive pulmonary disease (COPD) who were inactive agreed that sufficient exercise should be part of their daily life. Of this group, however, $44 \%$ indicated that they needed help to achieve this. ${ }^{10}$ Also, in people with type 2 diabetes, additional support seems to be needed to motivate and activate them. ${ }^{11}$ That is why physical activity counselling in primary health care is recommended for people with chronic diseases. However, primary health care providers need strategies to improve their ability to counsel patients effectively. ${ }^{12,13}$ New technologies can be applied to support healthcare interventions in all age groups. ${ }^{14-16}$ In the It's LiFe! study we developed a tool ${ }^{17}$ embedded in a Self-management Support Programme (SSP) that may support primary care professionals in their coaching role and patients with a chronic disease in improving their success in achieving an active lifestyle. The intervention helps to increase patients' awareness of the risks of inactivity behaviour, in combination with self-monitoring of behaviour, goal setting, action planning, discussing self-efficacy, and providing tailored feedback. The tool provides real-time feedback, on a smartphone application (app), about physical activity related to a personal goal. The tool also provides dialogue sessions about physical activity barriers and facilitators, a historic overview of activity behaviour, and feedback messages about the results. Furthermore, the tool supports the primary care professional in accomplishing the coaching role by providing the activity data and results of dialogue sessions of their patients on a website.

The tool and SSP will only be a successful e-health intervention if they are adapted to the needs and preferences of the end users. This was achieved by following a usercentred design process (UCD). ${ }^{18}$ An essential step in this process was a usability test. Testing for usability reduces errors, reduces the need for user training and user support, and improves acceptance by users, ${ }^{19}$ which will probably lead to better compliance with the intervention. The aim of the study reported in this paper was to test the usability of all parts of the It's LiFe! tool by end users (patients). 


\section{Methods}

Usability is defined as, "The extent to which a product can be used by specified users to achieve specified goals with effectiveness, efficiency and satisfaction in a specified context of use'. ${ }^{20}$ Indicators for effectiveness, efficiency, and satisfaction are error rate, task completion time, and a satisfaction rating questionnaire. ${ }^{21}$ In this study, usability was tested in a mixed-method approach in the following four phases:

1. a heuristic evaluation by experienced technology users and developers;

2. a usability test in a laboratory (lab) setting with end users;

3. a real-life pilot test by end users;

4. a second usability test in the lab with end users.

The medical-ethical committee of Maastricht University Medical Centre+ approved the studies.

\section{System description and use}

The It's LiFe! tool consists of three elements: ${ }^{17}$

1. an activity sensor with Bluetooth connectivity worn on the hip, clipped on the belt;

2. a smartphone (Samsung Galaxy Ace; Samsung Electronics Co., Seoul, South Korea) with an app for mobile feedback;

3. a web client for comprehensive feedback and data entry for patient and practice nurse.

Navigation through the smartphone works by swiping. For study phases 1, 2, and 4, dummy data were available on the phone and website. Three types of feedback are provided by the tool (Figure 1). The first feedback loop contains the real-time activity data compared to a personal goal on a widget and in the menu of the app, per hour, day, week, and month. The second feedback loop consists of dialogue sessions and feedback messages based upon the activity results. These are generated by the system and accessible from the app and the patient's website. Dialogue sessions consist of questions regarding the barriers facilitators and patients face in becoming active, preparatory questions for setting an activity goal, and advice for action planning. The third feedback loop is the feedback from the practice nurse during consultations. In these consultations, motivational interviewing, risk communication, and goal setting are used as counselling techniques. Figure 2 provides an overview of the entire intervention executed in the pilot.

\section{Participants}

For the heuristic evaluation (phase 1), six people were selected who were known for their experience with the development, evaluation, or extensive use of technology (technology experts). End users were people with COPD or type 2 diabetes, aged 40-70 
years, and familiar with the Dutch language. In phases $2-4$, these criteria were used to select participants. For the laboratory tests (phases 2 and 4), eleven patients were invited through an invitation letter. For the pilot in real life (phase 3), 20 patients were invited by practice nurses in two participating general practices.

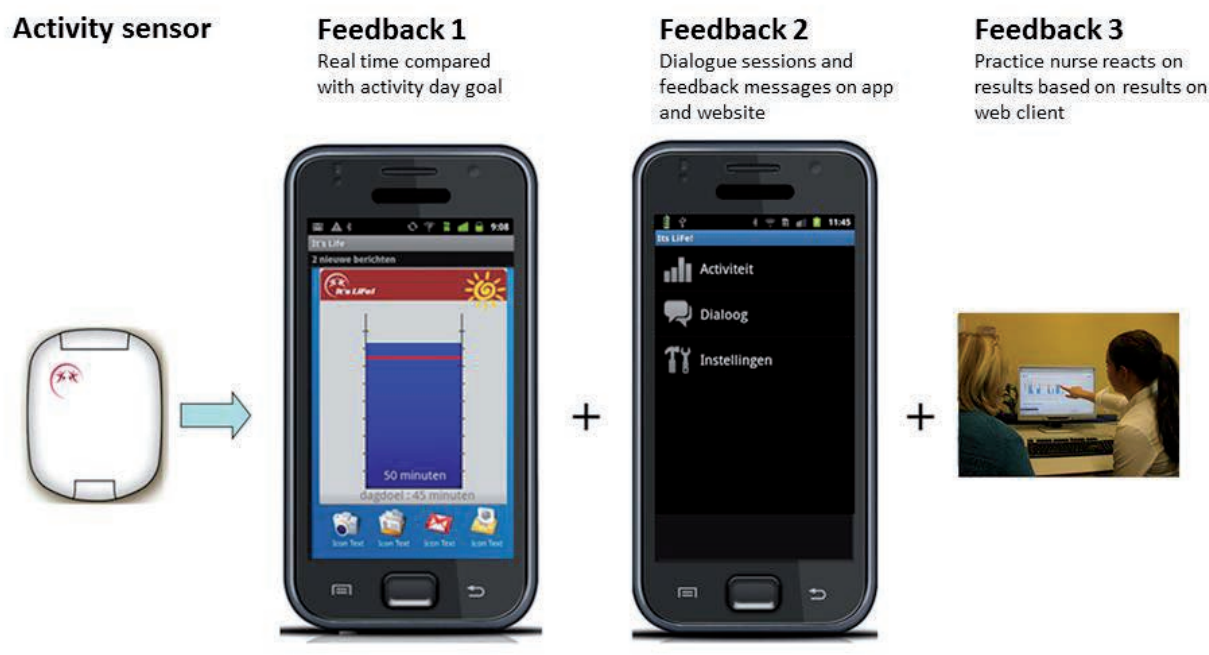

Figure 1 The It's LiFe! tool.

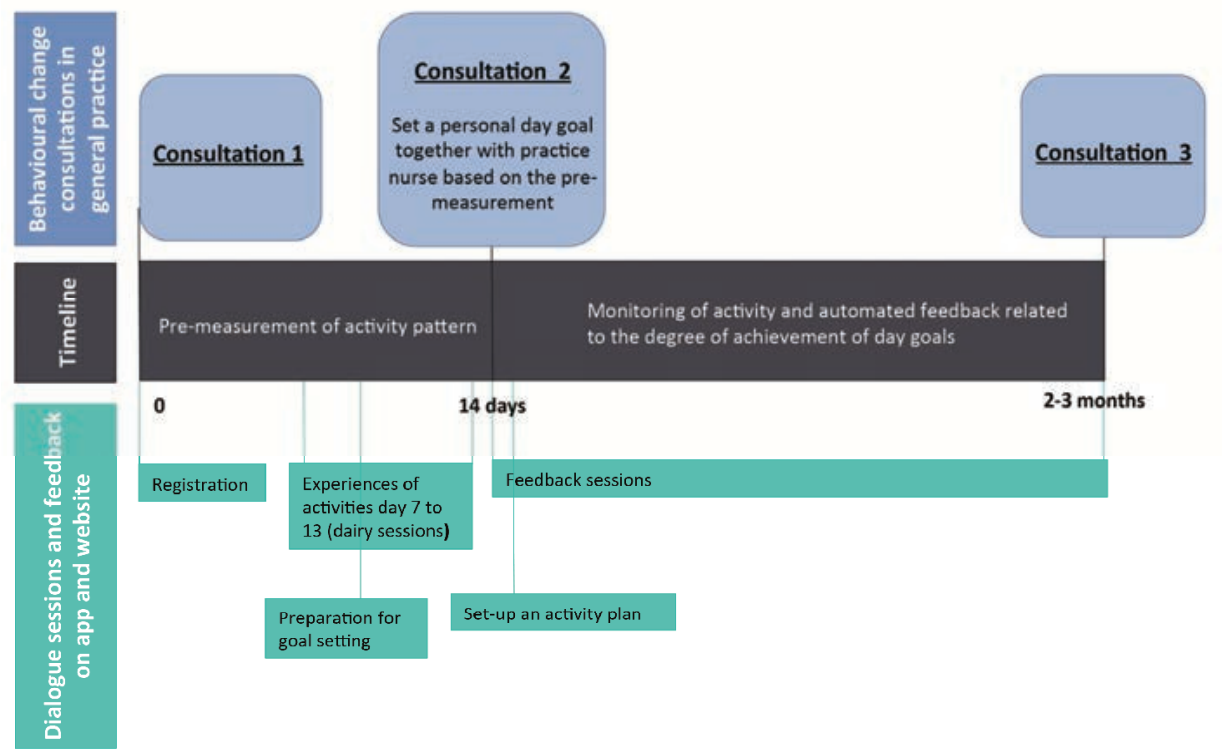

Figure 2 Timeline of the behavioural change consultations with practice nurse and dialogue sessions during the pilot (phase 3). 


\section{Study design}

Below, the four study phases are described in detail.

\section{Phase 1: Heuristic evaluation by experts}

The heuristic evaluation was based on Nielsen's 10 usability principles. ${ }^{22}$ These principles concern, among others, language use, error prevention, consistency, and efficiency of use. The test was performed by six technology experts. Each evaluator started by reading the manual. They were asked to write down any suggestions for improvement and thereafter to use the interface on the app twice - first to obtain a general idea about the app and then to go in depth for each screen - write down remarks, and score the ten usability heuristics on a scale from 1 (very bad) to 7 (very good). A heuristic was interpreted as violated by a score of 4 or lower. After completing the evaluation, the experts discussed their comments and scores with the researcher. The results of this phase were used to adjust the manual and develop a second prototype of the app.

\section{Phase 2: Usability test in a laboratory setting by patients}

In phase 2, a think-aloud procedure was performed by end users to evaluate the usability of the manual, app, and server. For the dialogue sessions, the researcher noted which tasks the participants completed: participants were free to complete the session from the server on the app or the website. Before the actual test, the participants completed a questionnaire that included questions regarding birth year, level of education, kind of mobile phone, and Internet use in hours per week. Participants rated the manual for comprehensiveness and readability on a scale from 1 (very bad) to 7 (very good). Then, after a short explanation, the participants individually performed seven predetermined tasks on the app and completed three to seven dialog sessions (depending on the time left). The users were asked to verbalize their thoughts while performing these tasks. Tasks were formulated in such a way as to guarantee that all functionalities of the interfaces were used and tested. All tasks are presented in Table 1. Participants were observed by a researcher throughout their task performance. The researcher registered all users' comments during task performance, including the need for assistance, the number of errors, and expressed suggestions for improvement. The researcher also registered relevant nonverbal communication (e.g. confident or confused facial expressions). In addition, participants' facial expressions, audio, on-screen activity, and keyboard/mouse input were videotaped with Morae Recorder (version 3.1.1; TechSmith Corporation, Okemos, MI, USA).

Participants valued the complexity of each task on a scale from 1 (very difficult) to 7 (very easy). Dialogue sessions were also rated for comprehensiveness and readability on a scale from 1 (very bad) to 7 (very good). Furthermore, all participants completed the translated post-study system usability questionnaire (PSSUQ). The PSSUQ consists of 19 items that are rated on a 7-point scale (strongly disagree [1] to strongly agree). ${ }^{23}$ The 
PSSUQ consists of an overall satisfaction scale and three subscales: system usefulness (items 1-8); information quality (items 9-15); and interface quality (items 16-18). Higher scores indicate better usability. Missing data were interpolated by averaging the remaining domain scores. ${ }^{23}$ One item was not included as it was not applicable for the app or for the website. The PSSUQ was completed twice, once for the app and once for the website. The prototype was adapted on the basis of the results of this test.

Phase 3: Pilot test in real life

In phase 3 , usability was tested by end users in real life, which implies that the tool was used in daily life (at home, at work, etc) and embedded in primary care. A practice nurse provided the tool to the patient in a first consultation which was aimed at behavioural change. ${ }^{24}$ Subsequently the patients wore the tool for 2 weeks to get a baseline measurement of their physical activity. Patients received dialogue sessions on the app and website with questions about barriers and facilitators for physical activity. Every day, the results were automatically sent to the practice nurses' website.

After the baseline measurement, a consultation took place in which the patient and practice nurse set an activity goal in minutes per day. Thereafter, the patients continued wearing the tool for another 10 weeks. They composed an activity plan in a dialogue session and received feedback from the tool about their performance compared with their personal goal. In a final consultation, 3 months after the start of the intervention, the practice nurse reflected on the activity results in a final consultation. Instructions on using the tool were given in a written manual and instruction movies were available on YouTube.

After each consultation, participants were interviewed about their experiences. The interviews were audio taped and transcribed. At the end of the intervention period, participants completed the Software Usability Measurement Inventory (SUMI) questionnaire. The SUMI contains 50 items that have to be answered on a 3-point Likert scale (agree, undecided, disagree). The SUMI consists of a global scale and five subscales (efficiency, affect, helpfulness, control, and learnability). ${ }^{25}$ The subscales are all linked to questions throughout the SUMI questionnaire. Software usability is considered reasonable with scores of 50 or more on each of the scales. ${ }^{26}$ In addition, the number of errors, technical failures, defects, and causes of the defects were collected in logbooks kept by a helpdesk and the end users, including the practice nurses.

\section{Phase 4: Usability test in a laboratory setting by patients}

After the pilot test, the prototype was further improved. To ensure that the latest adaptations did not introduce new problems, the laboratory usability test was repeated with five new end users. To measure satisfaction, Microsoft's desirability toolkit (Microsoft Corporation, Redmond, WA, USA) ${ }^{27}$ was added to the protocol. From a list of 118 words ( $60 \%$ with a positive and $40 \%$ with a negative meaning), participants were first asked to mark all words they found applicable to the system (app and website separately). Sec- 
ond, they were asked to choose from the selected words the five that most closely matched their personal reactions to the system, and to explain their choice. The desirability toolkit was translated into Dutch by two independent researchers.

\section{Statistics}

For the quantitative measurements (baseline characteristics, Likert-scale questionnaires, PSSUQ questionnaire), means and standard deviations were calculated using SPSS software package 19 (IBM Corporation, Armonk, NY, USA). The SUMI data were analysed by the SUMI-service using the proprietary software SUMISCO (Human Factors Research Group, University College, Cork, Ireland).

Missing values were scored as "undecided". Lists with more than four missing values were left out of the analysis. Qualitative data collected during the observations in the lab and in the real-life test were recorded, summarized, and analysed with a directed content analysis. ${ }^{28}$

\section{Results}

\section{Heuristic evaluation}

The responses to Nielsen's heuristics indicated no major issues: all items scored on average 4 or higher. Help documentation could be improved by including information about the "back" and "on/off" buttons of the phone and not using the word "widget". According to some evaluators, the heuristic "visibility of the system status" was violated because it took too long for a session to open and there was no feedback about waiting time. Based on the results, the manual was rewritten and a new prototype was built with easier language, more consistency, other icons, extended swiping function to all screens, and an extended surface to the whole screen in the day view. The connectivity of the dialogue sessions was improved and indicators for progress and waiting time were added. There were several remarks about the term "sessions," but no better expression was found.

\section{Usability test in lab}

The new prototype was evaluated in a lab situation by four male patients and one female patient. The participants spent on average 21 hours a week on a computer. One participant had prior experience with a smartphone (Table 2). 
Table 2 Demographic characteristics of the patient participants

\begin{tabular}{|c|c|c|c|}
\hline Characteristics of the participants & $\begin{array}{l}\text { Phase } 2 \\
\text { Lab } 1(n=5)\end{array}$ & $\begin{array}{l}\text { Phase } 3 \\
\text { Pilot }(n=20)\end{array}$ & $\begin{array}{l}\text { Phase } 4 \\
\text { Lab } 2(n=5)\end{array}$ \\
\hline Mean age (SD) & $61.4(8.5)$ & $60.2(9.0)$ & $58.6(7.8)$ \\
\hline \multicolumn{4}{|l|}{ Sex Number (\%) } \\
\hline Male & $4(80 \%)$ & $11(55 \%)$ & $3(60 \%)$ \\
\hline Female & $1(20 \%)$ & $9(45 \%)$ & $2(40 \%)$ \\
\hline \multicolumn{4}{|l|}{ Disease Number (\%) } \\
\hline COPD & $2(40 \%)$ & $10(50 \%)$ & $1(20 \%)$ \\
\hline Diabetes type 2 & $3(60 \%)$ & $10(50 \%)$ & $4(80 \%)$ \\
\hline \multicolumn{4}{|l|}{ Education level Number (\%) } \\
\hline$\leq$ intermediate vocational education & $3(60 \%)$ & - & $4(80 \%)$ \\
\hline >intermediate vocational education & $2(40 \%)$ & - & $1(20 \%)$ \\
\hline \multicolumn{4}{|l|}{ Computer experience Number (\%) } \\
\hline$\leq 5$ h per week & $1(20 \%)$ & $2(10 \%)$ & $1(20 \%)$ \\
\hline$>5 \mathrm{~h}$ per week & $4(80 \%)$ & $18(90 \%)$ & $4(80 \%)$ \\
\hline \multicolumn{4}{|l|}{ Smartphone experience Number (\%) } \\
\hline Yes & $1(20 \%)$ & $6(30 \%)$ & $1(20 \%)$ \\
\hline No & $4(80 \%)$ & 14 (70\%) & $4(80 \%)$ \\
\hline
\end{tabular}

The participants rated "comprehensiveness" and "readability" of the manual with an average of 4.5 and 4.8, respectively, on a scale ranging from 1 to 7 (Table 1). Based on the suggestions for improvement, the manual was extended with more information about the general use of the smartphone. One participant doubted whether the activity sensor was robust enough.

Although most participants had no previous experience with smartphones, none of the eight tasks on the app were rated as difficult (Table 1). Except in the case of opening the app for one person, no navigation errors were observed. The observers had to give only minor instructions about swiping and opening/returning to the app and widget. Results from the PSSUQ showed that the participants were, overall, satisfied with the usability of the app (details are presented in Figure 3). 
Table 1 Results on task complexity, text comprehensiveness, and readability from tests in the laboratory setting

\begin{tabular}{|c|c|c|c|c|}
\hline \multirow[t]{2}{*}{ Manual } & \multicolumn{2}{|c|}{ Phase 2} & \multicolumn{2}{|c|}{ Phase 4} \\
\hline & $\overline{N^{1}}$ & Mean (SD) & $N$ & Mean (SD) \\
\hline Comprehensiveness & $\overline{4}$ & $4.5(2.1)$ & 5 & $6.2(1.3)$ \\
\hline Readability & 4 & $4.8(2.2)$ & 5 & $6.0(1.4)$ \\
\hline \multicolumn{5}{|l|}{ It's LiFe! app } \\
\hline \multicolumn{5}{|l|}{ Task complexity } \\
\hline You see the widget, what is the activity goal? & 5 & $6.2(1.0)$ & 5 & $6.6(0.5)$ \\
\hline $\begin{array}{l}\text { Widget. Say out loud how many minutes of activity this person } \\
\text { had today }\end{array}$ & 5 & $7.0(0.0)$ & 5 & $7.0(0.0)$ \\
\hline Go to the It's LiFe! app & 5 & $5.8(0.1)$ & 5 & $5.8(1.8$ \\
\hline $\begin{array}{l}\text { Press 'activity,' press 'hour,' and scroll through the days by } \\
\text { swiping }\end{array}$ & 5 & $5.8(1.1)$ & 5 & $6.8(0.4)$ \\
\hline $\begin{array}{l}\text { Press 'day,' say out loud how many minutes this person } \\
\text { performed moderately intense activities and very intense } \\
\text { activities, three days ago }\end{array}$ & 5 & $5.6(1.1)$ & 5 & $5.6(1.7)$ \\
\hline How many days did this person reach his goal in week 3 & 5 & $5.6(2.1)$ & 5 & $6.8(0.4)$ \\
\hline How many days did this person reach his goal in month $X$ & 5 & $6.2(0.8)$ & 5 & $6.6(0.5)$ \\
\hline Average of all tasks & & $6.0(0.5)$ & & $6.5(0.5)$ \\
\hline \multicolumn{5}{|l|}{ It's LiFe! server } \\
\hline \multicolumn{5}{|l|}{ Task complexity } \\
\hline Go to the website and login & $3 w$ & $6.3(0.6)$ & $5 w$ & $6.6(0.5)$ \\
\hline Fill out a reminder & $3 w$ & $4.7(2.1)$ & 0 & - \\
\hline \multirow[t]{2}{*}{ Registration session } & $2 a$ & $6.5(0.7)$ & $5 w$ & v $5.4(1.5)$ \\
\hline & $2 w$ & $6.5(0.7)$ & & \\
\hline \multirow[t]{2}{*}{ Diary sessions } & $2 a$ & $5.7(1.2)$ & $2 a$ & $7.0(0.0)$ \\
\hline & $1 w$ & & & \\
\hline \multirow[t]{2}{*}{ Preparation for goal setting } & $3 a$ & $4.0(2.2)$ & $5 w$ & $6.0(0.7)$ \\
\hline & $2 w$ & & & \\
\hline Set up activity plan & 3 & $6.0(1.0)$ & $5 w$ & $6.0(0.7)$ \\
\hline \multirow[t]{2}{*}{ Remarks of the day } & $1 a$ & $6.0(1.4)$ & $3 a$ & $6.0(1.0)$ \\
\hline & $1 \mathrm{w}$ & & & \\
\hline Average of all tasks & & $5.75(0.9)$ & & $6.2(0.6)$ \\
\hline \multicolumn{5}{|l|}{ Comprehensibility of the text per task } \\
\hline Register session & 4 & $5.0(2.0)$ & 5 & $6.0(0.7)$ \\
\hline Diary sessions & 3 & $6.3(1.2)$ & 2 & $7.0(0.0)$ \\
\hline Preparation for goal setting & 4 & $4.5(3.0)$ & 5 & $6.6(0.5)$ \\
\hline Set up activity plan & 3 & $6.3(0.6)$ & 5 & $6.4(0.9)$ \\
\hline Remarks of the day & 2 & $5.5(0.7)$ & 3 & $6.3(0.6)$ \\
\hline Watch 'the activity picture' & 1 & $6.0(0.0)$ & 5 & $6.8(0.4)$ \\
\hline Average comprehensibility of the text & & $5.6(0.7)$ & & $6.5(0.4)$ \\
\hline \multicolumn{5}{|l|}{ Readability of the text per task } \\
\hline Register session & 4 & $6.3(0.5)$ & 5 & $6.0(0.7) v$ \\
\hline Diary session & 3 & $6.3(0.6)$ & 2 & $7.0(0.0)$ \\
\hline Preparation for goal setting & 4 & $4.3(2.8)$ & 5 & $6.6(0.5)$ \\
\hline Set up an activity plan & 3 & $6.7(0.6)$ & 5 & $6.6(0.5)^{\vee}$ \\
\hline Remarks of the day & 2 & $6.0(0.0)$ & 3 & $6.3(0.6)$ \\
\hline Watch 'the activity picture' & 1 & $6.0(0.0)$ & 5 & $6.8(0.4)$ \\
\hline Average readability of the text & & $5.9(0.8)$ & & $6.6(0.4)$ \\
\hline
\end{tabular}




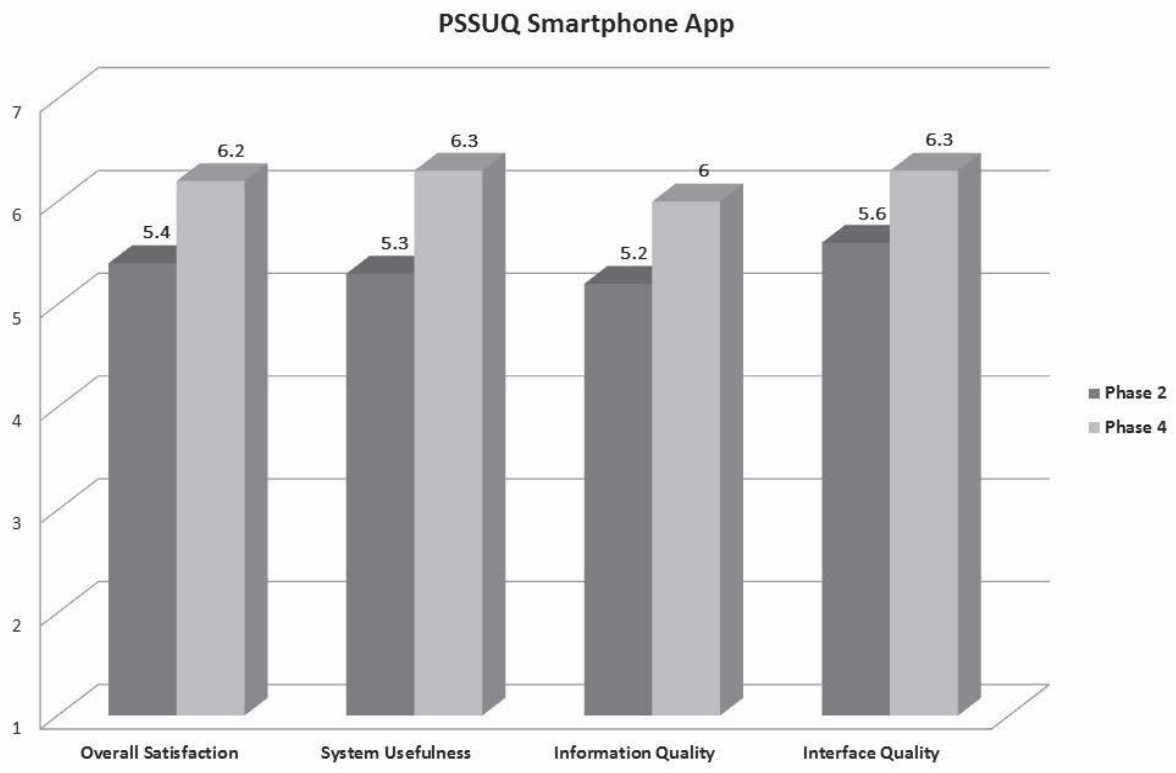

Figure 3 Post-study system usability questionnaire (PSSUQ) sub-scores for the smartphone app after phases 2 and 4.

Several suggestions for improvement were made during the thinking-aloud procedure. The most prominent suggestions included quicker response of the app on swiping, an always visible timescale in the hour view even when there is no activity, and better distinction between the bars in the month view. Those remarks were translated into improvements in the third prototype of the app.

The dialogue session "preparation for goal setting" was rated as the most difficult. Three out of four participants completed this task on the app. This task was a long session with different input methods per question. Based on the results of the lab tests, a recommendation was added that long and complex dialogue sessions should be completed via the website rather than via the app. These sessions were: "registration", "preparation for goal setting", and "set up an activity plan".

In relation to completing sessions on the website, it was observed that the "home" button of the website should be made more prominent, all monitoring results (results per hour, day, and week) visible on the app should also be visible on the web interface, and the intention of the "reminder" function should be more evident. Two out of three participants made errors while using this function. Furthermore, phrases like "you must" were perceived as paternalistic and should be changed to "you may". 
According to the PSSUQ (see Figure 4), participants were satisfied with the usability of the website. For all components of the tool, it seemed that participants with a higher education level were more critical than people with a lower education level.

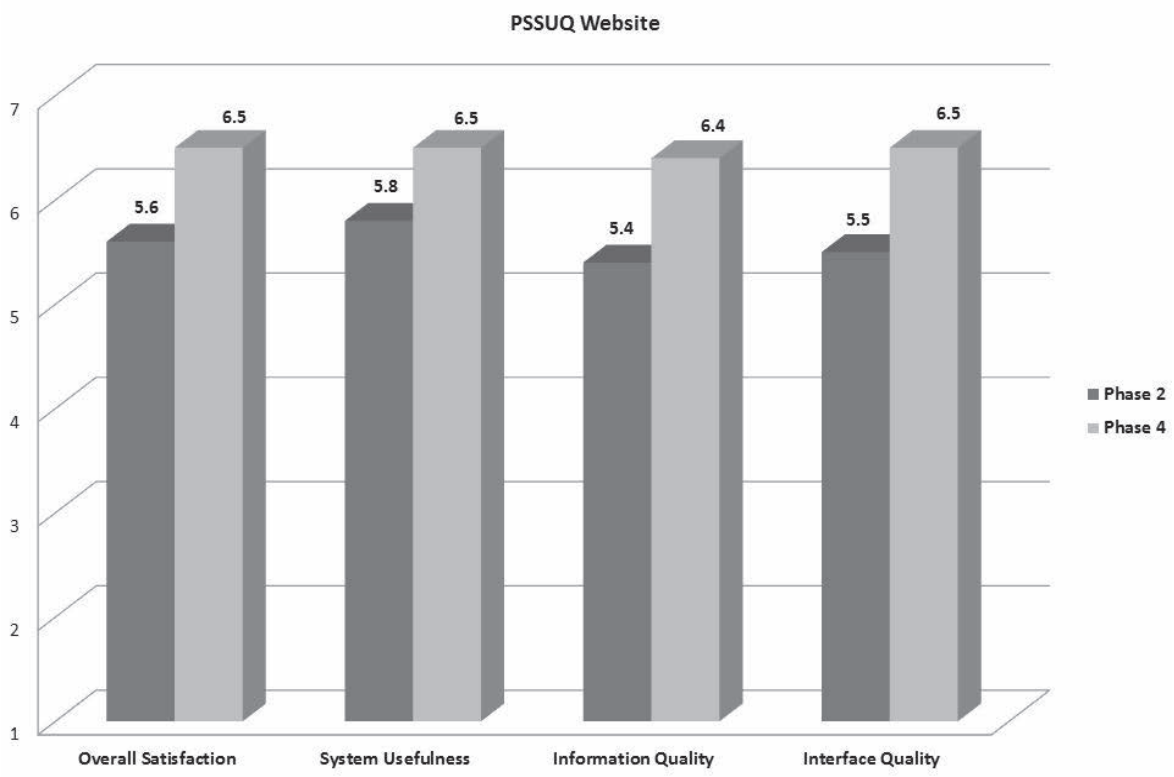

Figure 4 Post-study system usability questionnaire (PSSUQ) sub-scores for the website after phases 2 and 4.

All suggestions were incorporated in the next prototype of the tool, except for the suggestion to present all monitoring results via the web interface. This was not technically feasible.

\section{Pilot test in real life}

The third prototype was evaluated in a real life pilot by eleven men and nine women, with a mean age of 60.2 years (standard deviation 9.0 (Table 2). The data of the interviews and the log files were clustered into four themes: the sensor, data presentation, connection problems, and dialogue sessions.

\section{Activity sensor}

The participants had no difficulty wearing the activity sensor on a daily basis; however, most participants were afraid of losing the sensor. This problem was solved in the new prototype by adding a security clip with a thread that can be attached to belt loops. Two 
patients had to quit the pilot study because the hardware in the sensor broke, one of them because the participant accidentally put the sensor in the washing machine.

\section{Data presentation}

Most participants were positive about the tool in general. They liked to see the distance to their target goal and the course of activities over the day in the hour view. However, almost all participants had the idea that the activity results were not consistent with their experienced activity. This inconsistency had two causes:

1. A delay or failure in transmitting the activity data from the sensor to the phone. Therefore, besides the automatic transmission of data every 15 minutes, synchronization occurs when opening the activity menu and a refresh button has been added in the new prototype to actively synchronize the app with the sensor and server.

2. The activity sensor starts counting if the average acceleration per minute is approximately $\geq 3.5 \mathrm{~km} /$ hour and upper body movements are not captured. This was better explained in a new version of the manual and in the instruction movies. In addition, the practice nurse will have the ability to lower the threshold to 2 or $3 \mathrm{~km} /$ hour if participants are not able to reach the threshold noted above.

There were almost no comments on the usability of the app. In the day view, the word "moderate" was changed to "active" and "intense" to "active plus", since the word "moderate" was viewed as not encouraging. Furthermore, people were puzzled about the registered activity at 6 am, which seemed to be the summed activity and noise from midnight till 6 am. This has been solved by adding an "N" for night activity and raising the lowest threshold to separate noise from activity.

\section{Connection problems}

In order to make a connection between the phone and the server, participants had to log in on the phone once at the start of the intervention. Seven participants forgot to log in or did not manage to complete the task because they were not able to type in their correct user name and log-in on the phone. Based upon this, the registration session has been extended with a task to log in and an instruction on how to do this. Furthermore, the manual has been extended.

Seven patients complained that automatic data transfer from sensor to phone (which should occur every 15 minutes) did not work properly. It appeared that they had erroneously deactivated the smartphone's data connection. In addition, sometimes the Bluetooth connection failed because the sensor was out of range of the phone.

Due to the sleep mode of the phone and incorrect timings of data transmission, the connection between the phone and the server failed frequently, which meant that the patients and practice nurse did not see results on the website. This also meant that only two participants received more than one feedback message, since these messages depend on goal achievement and, therefore, the forwarded activity data. 


\section{SUMI Scale Profiles: Median Boxplots}

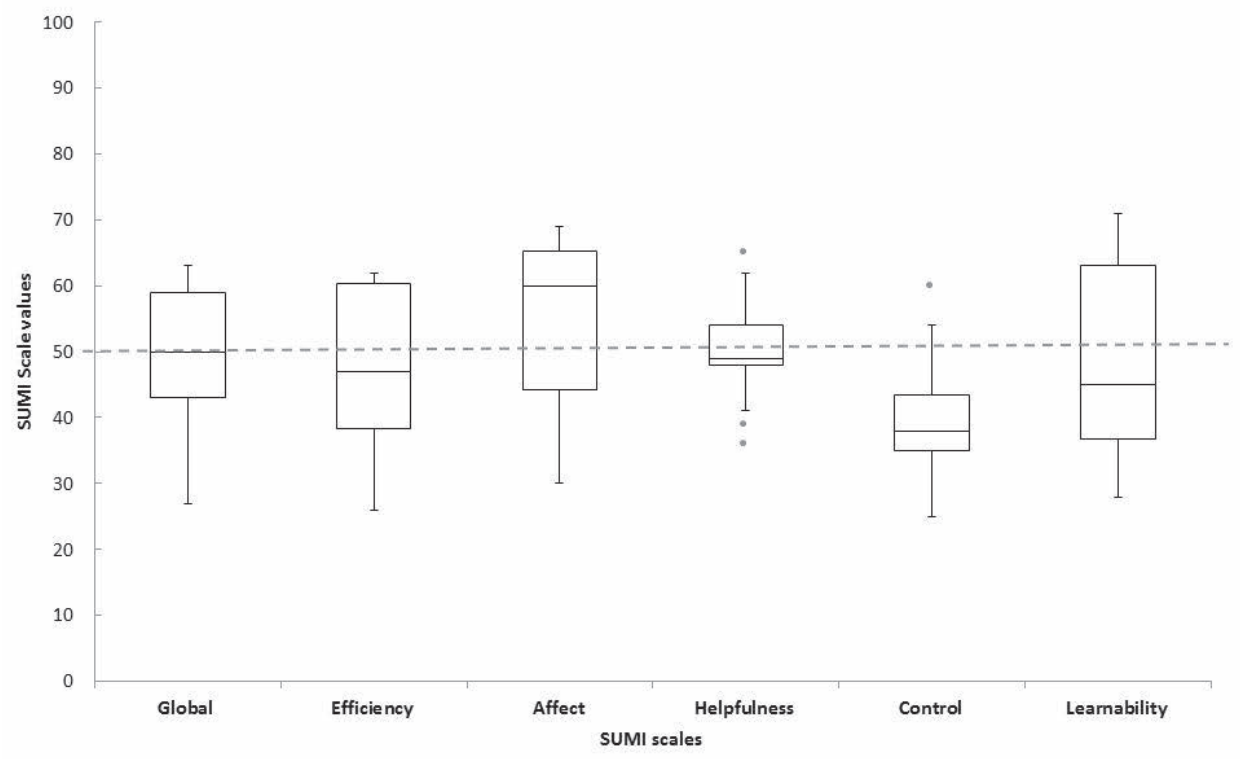

Figure 5 Results of the SUMI questionnaire, completed by 14 participants after the pilot in real life.

The dotted line represents the scale-midpoint.

\section{Dialogue sessions}

The participants did not give detailed feedback on the content of the sessions; however, the following suggestions for improvements were revealed. Participants were confused about the difference between the diary sessions and the "remarks of today" and, in their view, there were too many sessions. In response, the sessions were renamed, the session "remarks of today" was no longer announced by email, the diary sessions were offered less often, and some text fields were enlarged. Based upon these results, all errors were solved in a new prototype and the help documentation was extended, the manual and instruction movies were adapted, and all comments about ambiguities were merged in a "frequently asked questions" file.

The SUMI questionnaires of 14 participants were analysed (four did not fill out the questionnaire and two had more than four missing values). The results are presented in Figure 5. The score of 50 on the global scale indicates that satisfaction with the It's LiFe! tool is reasonable. The efficiency, helpfulness, control, and learnability could be improved. The only sub-score above average is "affect", which indicates that the users liked the interfaces and the idea of the tool. 


\section{Usability test in lab}

The fourth prototype was evaluated by three men and two women, with a mean age of 58.6 years (standard deviation 7.8 ), in a lab situation. Only one participant had experience with a smartphone.

The comprehensiveness and readability of the manual had clearly improved compared to the first usability test in the lab (phase 2), as is shown in Table 1. During the thinking-aloud procedure, the main suggestion made was to replace technical or English terms in the manual with easier terms or Dutch language.

All tasks on the app were scored as less or equally complex compared to the first lab test, few errors were observed, and difficulties were only faced with opening the app menu and the understanding of the terms "active" and "active + " for moderate and intense activities, respectively. According to the scores on the PSSUQ, user satisfaction with the usability of the app had also improved, as shown in Figure 3. During the thinking-aloud procedure, no suggestions for improvement were made regarding the app. The "registration session," which was rated as the easiest session in the first lab test, was now rated the most difficult session (Table 1). This could be explained by the fact that this session was extended with a procedure to prevent people from not logging in (one of the major issues in the real-life test). As a result, the "registration session" had become more complex: due to the small keyboard on the phone, typing errors were made and the backspace and symbol buttons were hard to find. In the final prototype, the procedure for logging in on the phone is explained extensively in the registration session and an instruction movie for this session has been made available.

Based upon the results of the thinking-aloud procedure during completion of the dialogue sessions on the website, some text was adapted, the activity plan could be filled in twice, the structure of the "compose activity plan" was changed, and the number of questions was lowered. Results from the PSSUQ (Figure 4) show that satisfaction with the usability of the website improved compared to the earlier version of the prototype used in phase 2 .

The participants rated the desirability of the app and website positively. To describe the app, three participants chose the phrase "easy to use" and two participants chose the words "motivating", "usable", "understandable", "useful", "suitable", and "accessible". The only chosen word that could be interpreted as negative was "business-like". Concerning the website, "accessible" was chosen three times and "clear", "interesting", "understandable", and "stimulating" were chosen by two participants (Tables 3 and 4). Again, people with higher education levels tended to be more critical. Figure 6 shows the final interfaces of the app. 


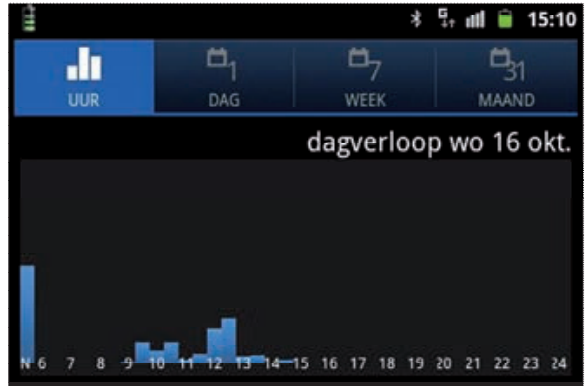

A. Day view per hour from 6 am to $12 \mathrm{pm}$. $\mathrm{N}$ indicates summed activity between midnight and $6 \mathrm{am}$.

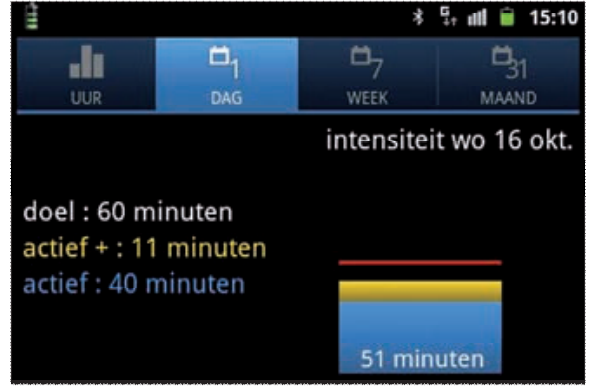

B. Day view.

The yellow parts indicate high intense activities, blue indicates moderate intense activities, the red line 'doel' is 'goal', 'minuten' is 'minutes'.

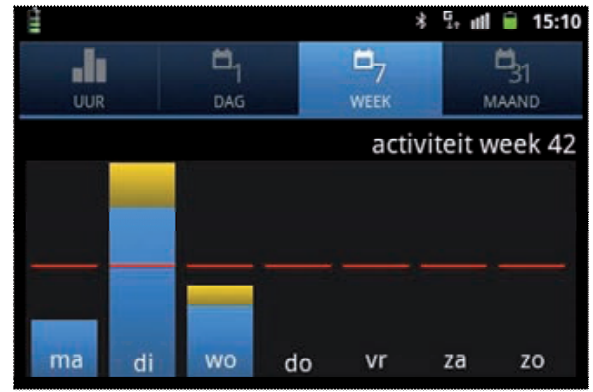

c. Week view.

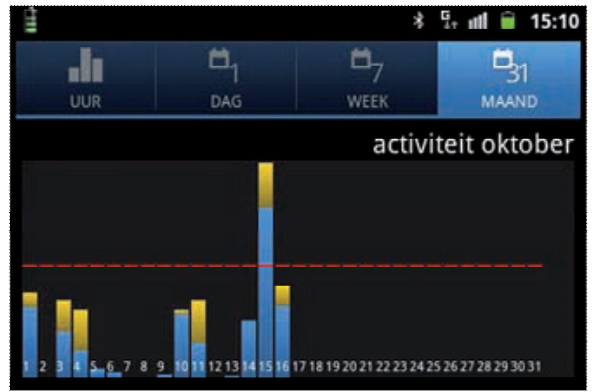

D. Month view.

Figure 6 Screenshots of the final prototype of the smartphone app.

Table 3 Desirability of It's LiFe! app

\begin{tabular}{|c|c|c|c|c|c|}
\hline $\begin{array}{l}\text { Participant } \\
\text { number }\end{array}$ & Desirability app & & & & \\
\hline 6 & advanced & fascinating & innovative & motivating & usable \\
\hline 7 & attractive & suitable & easy to use & motivating & understandable \\
\hline 8 & accessible & clear & easy to use & enthusiastic & useful \\
\hline 9 & accessible & comprehensive & easy to use & familiar & understandable \\
\hline 10 & business-like & suitable & straightforward & usable & useful \\
\hline
\end{tabular}


Table 4 Desirability of It's LiFe! website.

\begin{tabular}{llllll}
\hline Participant number & \multicolumn{2}{l}{ Desirability website } & & \\
\hline 6 & helpful & interesting & motivating & personal & stimulating \\
7 & accessible & calm & clear & comprehensive & understandable \\
8 & accessible & connected & comfortable & enthusiastic & reliable \\
9 & interesting & new & stimulating & understandable & useful \\
10 & accessible & approachable & clear & Innovative & suitable \\
\hline
\end{tabular}

\section{Discussion}

In response to the heuristic evaluation and tests in the laboratory and in real life, a new prototype of the It's LiFe! tool was developed. The usability of the tool improved during the study. The interface of the app needed relatively small adaptations. Most adaptations were made to the dialogue sessions on the phone involving the keyboard. In addition, connectivity problems were identified and solved.

The combination of laboratory and real-life tests and the combination of 'expertbased' and 'user-based' usability tests revealed a wide range of usability issues. ${ }^{19}$ The heuristic evaluation and the thinking-aloud procedure in the laboratory tests revealed the most valuable and detailed feedback on the interfaces and texts. The pilot in real life revealed practical issues such as connectivity problems and overall usability.

After the first test in the lab, usability was considered as good. The real-life test, however, revealed a whole different range of usability problems, and satisfaction with the It's LiFe! tool was low according to the SUMI results. This low appraisal was a logical consequence of the connectivity problems that occurred during the pilot. The high score on "affect" indicates that satisfaction with the interfaces and the idea of the tool was high, which is most likely due to the involvement of end users in the development process of the tool, the prior usability test in the laboratory, or because the participants liked the concept of the tool very much. It is possible that usability was rated more positive in the lab tests and in the interviews during the real-life test compared to the SUMI questionnaire because of social desirability bias. In other fields, it is observed that socially desirable answers are given more often in face-to-face interviews. ${ }^{29,30}$ People in our lab tests may have wanted to prove that they had the capabilities necessary to use the system or wanted to satisfy the researcher. ${ }^{31}$

All results should be considered with caution because of the small sample size. Nevertheless, it is known that tests with five participants are able to uncover $85 \%$ of usability issues. This number of evaluators is stated to be a good trade-off between completeness and investment ${ }^{32,33}$ Therefore, we think most usability issues have been revealed. 
This study shows the importance of a mixed-method approach, since different issues were revealed in the lab compared to the real-life test. The interfaces can be very effective, efficient, and desirable in a lab situation, but if communication fails between different components of the tool in a real-life situation, satisfaction will be low.

Almost all technical errors and suggestions for improvement have been incorporated in the newest version of the It's LiFe! tool. A crucial aspect that could not be handled is the need to log in on the phone in order to make a connection between the phone and the server. This is because privacy must be respected in all cases. Hopefully, the guidance provided by the instruction movie added to the registration session will be sufficient in further use. Furthermore, a hip-worn activity sensor has well-known restrictions, such as not capturing upper body movements. During the development process, the addition of another physiological measure was considered; however, this does not significantly improve the assessment of energy expenditure and reduces wearing comfort. $^{34}$ The effectiveness of the tool in combination with the SSP on physical activity level (exercise) will be tested in a randomized controlled trial.

\section{Acknowledgments}

This study was funded by the Netherlands Organization for Health Research and Development (ZonMw). Publication of the manuscript was supported by NWO, the Netherlands Organization for Scientific Research. We thank all patient participants and the practice nurses for sharing their time, thoughts, and experience with us, and especially the patient representatives, Jos Donkers and Ina van Opstal, for their remarks during the research meetings. Thanks to Trudy van der Weijden for her advice during the development process and Science Vision for the adaptations of the pictures.

\section{Disclosure}

The companies involved in the development are Maastricht Instruments BV, the Netherlands, IDEE Maastricht UMC+, the Netherlands, and Sananet Care BV, the Netherlands. The authors report no other conflicts of interest in this work.

\section{References}

1. Haapanen N, Miilunpalo S, Vuori I, Oja P, Pasanen M. Association of leisure time physical activity with the risk of coronary heart disease, hypertension and diabetes in middle-aged men and women. Int J Epidemiol. Aug 1997;26(4):739-747. 
2. Fentem $\mathrm{PH} . \mathrm{ABC}$ of sports medicine. Benefits of exercise in health and disease. Bmj. May 14 1994;308(6939):1291-1295.

3. Organisation WH. Global recommendations on physical activity for health. In: Organisation WH, ed. Geneva2010.

4. Haskell WL, Lee IM, Pate RR, et al. Physical activity and public health: updated recommendation for adults from the American College of Sports Medicine and the American Heart Association. Circulation. Aug 28 2007;116(9):1081-1093.

5. Schiller JS, Lucas JW, Ward BW, Peregoy JA. Summary health statistics for U.S. adults: National Health Interview Survey, 2010. Vital Health Stat 10. Jan 2012(252):1-207.

6. Craig R, Mindell J, Hirani V. Health Survey for England 2008. Volume 1: Physical activity and fitness. London: NHS Information Centre;2009.

7. Hildebrandt VH, Ooijendijk WTM, van Leven TK. Trendrapport bewegen en gezondheid 2008/2009: TNO, Kwaliteit van Leven, Bewegen en Gezondheid; 2010.

8. Long Alliantie N. Zorgstandaard COPD. Amersfoort: Long Alliantie Nederland; 2010.

9. Nederlandse Diabetes F. NDF Zorgstandaard : transparantie en kwaliteit van diabeteszorg voor mensen met diabetes type 2. Amersfoort: Nederlandse Diabetes Federatie; 2007.

10. Baan D, Heijmans M. Mensen met COPD in beweging. 2012.

11. Albright A, Franz M, Hornsby G, et al. American College of Sports Medicine position stand. Exercise and type 2 diabetes. Med Sci Sports Exerc. Jul 2000;32(7):1345-1360.

12. Carroll JK, Fiscella K, Epstein RM, et al. Physical activity counselling intervention at a federally qualified health center: improves autonomy-supportiveness, but not patients' perceived competence. Patient education and counselling. Sep 2013;92(3):432-436.

13. Hebert ET, Caughy MO, Shuval K. Primary care providers' perceptions of physical activity counselling in a clinical setting: a systematic review. Br J Sports Med. Jul 2012;46(9):625-631.

14. Bardsley M, Steventon A, Doll H. Impact of telehealth on general practice contacts: findings from the whole systems demonstrator cluster randomised trial. BMC health services research. 2013;13:395.

15. Price M, Yuen EK, Goetter EM, et al. mHealth: A Mechanism to Deliver More Accessible, More Effective Mental Health Care. Clinical psychology \& psychotherapy. 2013.

16. Esposito M, Ruberto M, Gimigliano F, et al. Effectiveness and safety of Nintendo Wii Fit Plus TM training in children with migraine without aura: a preliminary study.

17. van der Weegen S, Verwey R, Spreeuwenberg M, Tange H, van der Weijden T, de Witte L. The Development of a Mobile Monitoring and Feedback Tool to Stimulate Physical Activity of People With a Chronic Disease in Primary Care: A User-Centred Design. JMIR mhealth and whealth. 2013;1(2):e8.

18. Shah SG, Robinson I. Benefits of and barriers to involving users in medical device technology development and evaluation. Int J Technol Assess Health Care. Winter 2007;23(1):131-137.

19. Jaspers MW. A comparison of usability methods for testing interactive health technologies: methodological aspects and empirical evidence. Int J Med Inform. May 2009;78(5):340-353.

20. Standardization IOf, ISO. 9241-11, Ergonomic requirements for office work with visual display terminals (VDTs). Part 11: Guidance on usability1998.

21. Frøkjær E, Hertzum M, Hornbæk K. Measuring usability: are effectiveness, efficiency, and satisfaction really correlated? Paper presented at: Proceedings of the SIGCHI conference on Human factors in computing systems2000.

22. Nielsen J, Mack RL. Usability inspection methods. New York: Wiley; 1994.

23. Lewis JR. IBM computer usability satisfaction questionnaires: psychometric evaluation and instructions for use. International Journal of Human-Computer Interaction. 1995;7(1):57-78.

24. Verwey R, Weegen Svd, Spreeuwenberg M, Tange H, Weijden Tvd, Witte Ld. A pilot study of a tool to stimulate physical activity in patients with COPD or type 2 diabetes in primary care. J Telemed Telecare 2014;20(1):29-34.

25. Kirakowski J, Corbett M. SUMI: the Software Usability Measurement Inventory. British Journal of Educational Technology. 1993;24(3):210-212. 
26. Kirakowski J. Usability evaluation in industry. Chapter 19: The Software Usability Measurement Inventory. London; Bristol, Pa.: Taylor \& Francis; 1996.

27. Benedek J, Miner T. Measuring Desirability: New methods for evaluating desirability in a usability lab setting. Proceedings of Usability Professionals Association. 2002:8-12.

28. Hsieh H-F, Shannon SE. Three approaches to qualitative content analysis. Qualitative health research. 2005;15(9):1277-1288.

29. Waruru AK, Nduati R, Tylleskar T. Audio computer-assisted self-interviewing (ACASI) may avert socially desirable responses about infant feeding in the context of HIV. BMC Med Inform Decis Mak. 2005;5:24.

30. Luke N, Clark S, Zulu EM. The relationship history calendar: improving the scope and quality of data on youth sexual behaviour. Demography. Aug 2011;48(3):1151-1176.

31. Sauro J. 9 Biases in Usability Testing. 2012; http://www.measuringusability.com/blog/ut-bias.php. Accessed 18 November, 2013.

32. Nielsen J. Why You Only Need to Test with 5 Users. Evidence-Based User Experience Research, Training, and Consulting 2000; http://www.nngroup.com/articles/why-you-only-need-to-test-with-5-users/. Accessed 18 November, 2013.

33. Nielsen J, Landauer TK. A mathematical model of the finding of usability problems. Paper presented at: Proceedings of the INTERACT'93 and CHI'93 conference on Human factors in computing systems 1993.

34. Plasqui G, Bonomi A, Westerterp K. Daily physical activity assessment with accelerometers: new insights and validation studies. Obesity Reviews. 2013. 


\section{CHAPTER 4 \\ Concurrent validity of the MOX activity monitor compared to the ActiGraph GT3X}

This chapter was published as:

van der Weegen S, Essers H, Spreeuwenberg M, Verwey R, Tange H, de Witte L, Meijer K. Concurrent Validity of the MOX Activity Monitor Compared to the ActiGraph GT3X. Telemedicine journal and e-health : the official journal of the American Telemedicine Association. Apr 2015;21(4):259-266 


\section{Abstract}

Background The It's LiFe! monitoring and feedback tool embedded in primary care practice is promising in helping people to achieve an active lifestyle. This new tool consists of an activity monitor (the MOX), which is connected to a smartphone app and to a Web service for patients and care providers.

Aim Develop thresholds for the moderate and vigorous activity categories and examine the concurrent validity of the MOX in relation to the ActiGraph GT3X in healthy participants and chronically ill patients (Chronic Obstructive Pulmonary Disease and Type 2 diabetes) in a laboratory situation and during daily living.

Materials and Methods Participants wore the two activity monitors simultaneously on the lower back. An incremental treadmill protocol was executed by eight healthy adults and ten patients. For daily living measurements fifteen healthy adults and twelve patients wore the devices for 6-7 days. Daily living data were corrected for non-wear time, using diary information.

Results On the treadmill there was an excellent correlation between the ActiGraph and MOX counts (mean $r=0.99$ in healthy participants and mean $r=0.98$ in patients). Correlation during daily living was moderate (mean $r=0.72$ ) in healthy adults and good (mean $r=0.82$ ) in patients. Bland-Altman plots showed no perfect agreement between the two devices in minutes per category. However, a histogram of misclassified minutes showed that misclassification occurred around category thresholds.

Conclusions The MOX is capable of measuring physical activity and can be used in the It's LiFe! intervention. 


\section{Introduction}

Engagement in regular physical activity is effective in the primary and secondary prevention of several chronic diseases (e.g. cardiovascular disease, diabetes and some cancers) and reduces the risk of premature death. ${ }^{1,2}$ Globally, however, 31\% of the people above 15 years old are insufficiently active. ${ }^{3}$ Therefore numerous physical-activity-promoting interventions are developed. The most successful behavioural interventions include a mix of behaviour change techniques, such as goal setting, barrier identification, action planning, social support, self-monitoring of behaviour, revising of behavioural goals, and give advice and provide written materials. ${ }^{4-6}$ Technology can help to ease the implementation of behaviour change techniques in daily practice and can support health professionals in their coaching role. For example, pedometers in physical activity interventions, when used for self-monitoring seem to increase the daily steps significantly, especially when combined with goal setting. ${ }^{7}$ However, the ability of pedometers to assess freeliving activity is limited, due to the fact that they cannot measure the frequency, duration, and intensity of physical activity. ${ }^{8}$ Accelerometers do have these possibilities.

In the newly developed It's LiFe! intervention ${ }^{9-12}$, a monitoring and feedback tool embedded in a behaviour change counselling protocol supports patients in achieving an active lifestyle and gives care providers the possibility of using objective activity results while coaching. The purpose of the intervention is to support participants to increase the time spent in the moderate and vigorous activity category and diminish the time spent in the sedentary category. The It's LiFe! tool (Figure 1) consists of an accelerometer (the MOX), which is connected to a smartphone with Bluetooth. On both the smartphone and on a website users can see their minutes of activity in the moderate and vigorous category compared with their personal goal in minutes per day and receive feedback messages and behavioural change dialogue sessions. All feedback messages are in a positive tone and are based on personal goal achievement. Dialogue sessions make participants aware of the physical activities they prefer, barriers to overcome and guide the participant in activity planning and searching for social support. What makes the It's LiFe! tool unique is that it is developed in a user centred design process together with patients and care providers ${ }^{9}$ and that the activity results and answers to dialogue sessions are automatically sent to the care provider. The care provider uses the information to set an appropriate activity goal together with the patient, reinforce awareness, motivation and support for the behaviour change. Currently this coaching role is executed by a practice nurse in primary care.

To make use of the full potential of the user centred design approach it was necessary that all features of the tool were adaptable. Therefore a new activity monitor (the MOX) was developed which allowed adaptation of the algorithm to the preferences of the end users and could communicate with other systems.

For an effective intervention with sufficient adherence from users it is necessary that the tool provides accurate activity results. Methods to validate accelerometers are: 
against doubly labelled water (DLW), (video) observation, indirect calorimetry or another accurate accelerometer. DLW is expensive and merely gives information about energy expenditure rather than the distribution of activity bouts over time, and indirect calorimetry and video observation are only suitable in a laboratory setting. Therefore, we chose to evaluate the MOX in relation to another accelerometer, the ActiGraph GT3X. The ActiGraph GT3X is one of the most widely used and validated activity monitors in researcher environments ${ }^{13-15}$ and correlates as one of the best with indirect calorimetry $(r=0.77)^{16}$ and DLW $(r=0.68) .{ }^{17}$

The goal of the current study was twofold. First, thresholds for different activity intensities for the MOX were determined. Second, the It's LiFe! activity monitor, the MOX, was validated against the ActiGraph GT3X. This was tested on a treadmill and in daily life. This was conducted in both healthy adults and in different patient populations since activity monitor output can differ between populations, due to a difference in walking patterns. ${ }^{18}$

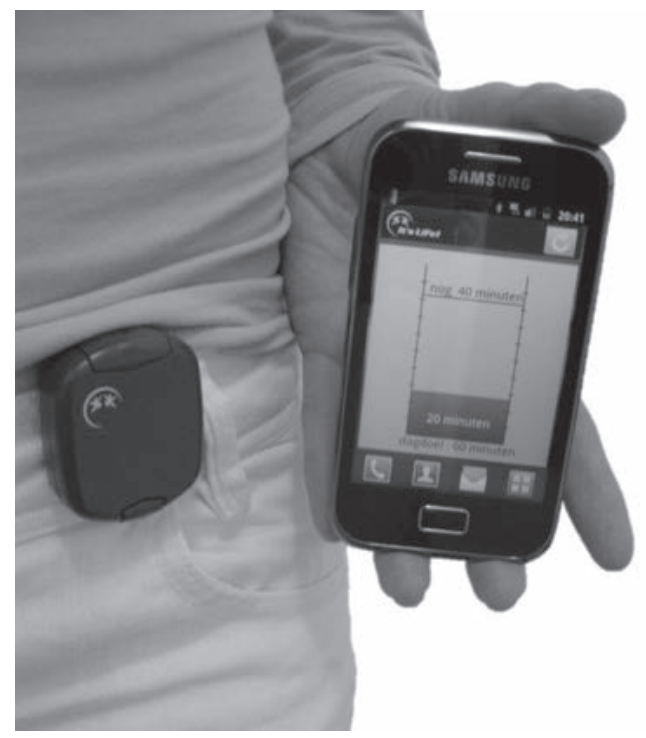

Figure 1 The It's LiFe! monitoring and feedback tool. The MOX and the real-time feedback (activity results in minutes a day compared with a personal goal) on the smartphone app are shown.

\section{Materials and methods}

\section{Study design}

The following methods were used for the multiple purposes of this study. 
1. Thresholds of the MOX were defined for different intensity levels (moderate and vigorous) by analysing the data of five healthy participants who wore the MOX and the ActiGraph simultaneously during daily living. Thresholds for these categories were defined because feedback of the tool is aimed at these categories.

2. The concurrent validity of the two monitors was defined and the thresholds of the MOX were validated by the data of an incremental treadmill protocol by eight healthy adults and ten participants with Chronic Obstructive Pulmonary Disease (COPD) or Type 2 diabetes and data of a second group of ten healthy participants and twelve people with COPD or type 2 diabetes during daily living.

\section{Participants}

Twenty-two healthy volunteers (18-70 years of age) were recruited from the researchers' local networks by an invitation letter. Thirteen volunteers with COPD or Type 2 diabetes (40 years or older), who were able to walk independently, were recruited by patient representatives from the national patient associations and by a practice nurse through an invitation letter. One healthy participant and nine patients contributed to both the measurements on the treadmill and during daily living. Written informed consent was obtained from all participants before study initiation. Confidential processing of data and anonymity were guaranteed. Participants had the opportunity to withdraw from the study at any time. The Medical Ethical Committee of MUMC+ approved this study.

\section{Activity monitors}

The MOX, (model MMOXX1.01), is an activity monitor $(4.5 \mathrm{~cm} \times 4.0 \mathrm{~cm} \times 1.4 \mathrm{~cm})$ with a sample frequency of $25 \mathrm{~Hz}$. The MOX measures, in 3-axis, the acceleration data $( \pm 6 \mathrm{G})$. The data are filtered with a Butterworth $0.1 \mathrm{~Hz} 4^{\text {th }}$ order high-pass filter. These data are converted to activity counts via embedded software by integrating the acceleration over one-minute episodes and summing this outcome for all 3 axes. Subsequently the data are calibrated so that a constant acceleration of $1 \mathrm{G}$ over a minute corresponds to 1000 counts. ${ }^{19}$ The ActiGraph GT3X is a 3 -axis activity monitor $(3.8 \mathrm{~cm} \times 3.7 \mathrm{~cm} \times 1.8 \mathrm{~cm})$ with a sample frequency of $30 \mathrm{~Hz}$, which provides activity data in a scale from 0 to 15.000 counts per minute. In both devices the counts were recorded in the internal memory housed in the monitor and transferred to a computer with a micro USB. For the ActiGraph the programme ActiLife v5.10.0 was used, whereas for the MOX, MOXBWO software was used. In the It's LiFe! intervention the MOX will transfer its data automatically with Bluetooth to a smartphone. 


\section{Data collection procedures}

Throughout testing, the two activity monitors were worn simultaneously next to each other with an elastic belt tightly secured at the lower back. The devices were synchronized with the time on the same computer and batteries were charged by the researchers before distribution, as the devices were able to monitor for 8 days. People with diabetes Type 2 were asked to report their last measured HbA1c and people with COPD their last spirometry results.

\section{Treadmill}

All participants wore normal clothes (with a restriction of high heels) with the MOX and the ActiGraph on their lower back. For the healthy participants the treadmill started at 3 $\mathrm{km} / \mathrm{h}(1.9 \mathrm{mph})$ and became faster every minute by $1 \mathrm{~km} / \mathrm{h}(0.6 \mathrm{mph})$ until the participant indicated they wanted to stop. Maximum speed was $11 \mathrm{~km} / \mathrm{h}$ (6.8 mph). The participants with a chronic disease started at a pace of $2 \mathrm{~km} / \mathrm{h}(1.2 \mathrm{mph})$. The treadmill speed was increased every three minutes by $0.5 \mathrm{~km} / \mathrm{h}(0.3 \mathrm{mph})$ until the patients indicated they wanted to stop, to prevent overexertion.

\section{Monitoring of activities during daily living}

The activity pattern during daily life was measured for 6-7 consecutive days during waking hours, except for showering, swimming- or other water activities. The participants were asked to exhibit their normal daily activities and to keep a diary to report at what time they wore the devices.

\section{Statistical analysis}

All analyses were performed using IBM Statistical Product and Service Solutions (SPSS version 20.0). Graphs were conducted in Microsoft Excel 2010. Bland-Altman plots were made with MedCalc version 12.5.0.0.

\section{Define thresholds}

Thresholds for moderate and vigorous intensity activities has been defined for the ActiGraph14: moderate 2690-6166 counts (3.0-6.0 METS) and vigorous $>6167$ counts ( $>6.0$ METS). The activity categories of the ActiGraph were used as a standard to define the thresholds for the activity categories of the MOX. To this end, agreement of allocating activities to either of these categories for the two devices was tested by making small incremental steps in the MOX thresholds (i.e. 10 MOX counts) until the smallest absolute difference was obtained between minutes spent per category between the two devices. 


\section{Concurrent validity}

For the treadmill measurements Pearson correlation per person was calculated between speed and MOX counts per minute, and ActiGraph and MOX counts per minute. To account for the dependency of measurements of speed within participants, the association between MOX counts and speed (dependent variable) and MOX counts and ActiGraph counts per minute (dependent variable) were analysed using multilevel, linear, mixed model analyses with speed at level 1 and participant at level 2 factors. Akaike's information criterion (AIC) was used to choose the best model. For daily living measurements Spearman correlation was calculated between the counts per minute of the Actigraph and the MOX per person per day. Values over 0.8 were rated as good. To prevent an inflated high correlation between the devices due to non-wear time during daily life measurements, periods of non-wear time were omitted based on diary information from the participants. Agreement between minutes per category (moderate and vigorous intensity activities) measured by the different devices was analysed using Bland-Altman plots for repeated measures. The mean values and difference with the ActiGraph were plotted, and the systematic bias and limits of agreement (LOA) (within mean \pm 2 SD of the mean differences) were obtained. To gain further insight into misclassified minutes, histograms of the counts of these minutes were obtained.

\section{Results}

\section{Participants characteristics threshold definition}

The first group of five healthy participants had a mean age of $33.4 \pm 11.6$ years. The mean height and weight were $181.0 \pm 10.1 \mathrm{~cm}$ and $75.7 \pm 12.3 \mathrm{~kg}$ respectively. The participants wore the devices on $6.8 \pm 0.8$ days.

\section{Participant characteristics validation}

Participant characteristics are presented in Table 1.

\section{Treadmill}

Eight healthy participants and five people with COPD and five people with diabetes Type 2 walked on the treadmill. The data of one patient with COPD could not be used since this person was not able to walk on the treadmill for three minutes without resting.

\section{Daily living}

The 10 healthy participants spent on average 26.1 (SD 18.0) minutes in the moderate category and 4.4 (SD 13.2) in the vigorous category, as determined by the ActiGraph. Out of 67 days, the ActiGraph did not record data on seven days and on two days the 
battery of the MOX was empty. Among the participants with a chronic disease, five participants had Type 2 diabetes and seven suffered from COPD. Patients were active for 11.6 (SD 13.1) minutes in the moderate category and 0.7 (SD 3.9) minutes in the vigorous category. In patients the ActiGraph did not record data on 14 out of 89 days.

Table 1 Participant characteristics validation

\begin{tabular}{lll}
\hline Treadmill & Healthy $(\mathrm{n}=8)$ & Patients $(\mathrm{n}=9)$ \\
\hline Age (in years) & $24.1(5.3)$ & $60.9(7.1)$ \\
COPD/DM2 $(\mathrm{n})$ & - & $4 / 5$ \\
Height $(\mathrm{cm})$ & $183.3(9.4)$ & $170.9(11.4)$ \\
Weight $(\mathrm{kg})$ & $75.1(13.4)$ & $92.4(27.0)$ \\
BMI $\left(\mathrm{kg} / \mathrm{m}^{2}\right)$ & $22.2(2.5)$ & $31.3(7.5)$ \\
FEV1 $(\%$ predicted) & - & $52.5(16.6)(\mathrm{n}=4)$ \\
Hba1c (mmol/mol) & - & $52.2(6.0)(\mathrm{n}=5)$ \\
\hline Daily living & Healthy $(\mathrm{n}=10)$ & Patients $(\mathrm{n}=12)$ \\
\hline Age (in years) & $30.4(8.3)$ & $61.6(9.2)$ \\
COPD/DM2 (n) & - & $5 / 7$ \\
Height (cm) & $175(9.6)$ & $168.6(9.9)$ \\
Weight (kg) & $68.1(8.9)(\mathrm{n}=9)$ & $95.6(31.5)$ \\
BMI (kg/m $\left.{ }^{2}\right)$ & $22.7(2.8)$ & $33.3(9.8)$ \\
Valid wear days & $5.8(1.2)$ & $6.3(1.4)$ \\
Wear time & $746.4(191.3)$ & $854.0(174.6)$ \\
(minutes per day) & & \\
FEV1 (\% predicted) & - & $50.5(12.6)(\mathrm{n}=4)$ \\
Hba1c (mmol/mol) & - & $53.1(6.4)(\mathrm{n}=7)$ \\
\hline FEV Forced expiratory & &
\end{tabular}

FEV Forced expiratory volume

BMI Body Mass Index

\section{Define thresholds}

The best possible agreement between the activity classification of the ActiGraph and the MOX was reached with the following thresholds for the MOX: moderate 400-860 and vigorous 860 - $\infty$ counts per minute. These thresholds were used to define the minutes of activity in the different intensity levels during the daily living of the patients.

\section{Concurrent validity}

Treadmill.

MOX activity counts per minute increased with the intensity of walking in healthy participants mean $r=0.98$ (range $0.96-0.99$ ) and in patients mean $r=0.99$ (range $0.98-1$ ). Linear mixed model analyses showed that MOX counts significantly increased with speed in healthy participants and in patients (both $\mathrm{p}<0.00$ ) (Figures 2 and 3). Pearson correlation between the ActiGraph and the MOX counts per minute was good. In healthy participants mean $r$ was 0.99 (range 0.98 - 1.00). In patients mean $r$ was 0.98 (range 0.95 - 
1.00). Linear mixed model analyses showed that in healthy participants and in patients MOX counts significantly increases with ActiGraph counts (in both groups $p<0.00$ ). Figures 2 and 3 show that the threshold for moderate intense activities lies between 3-4 $\mathrm{km} / \mathrm{h}$ and that the variance in counts increases with speed. The increase in variance with speed is also observed in ActiGraph counts.

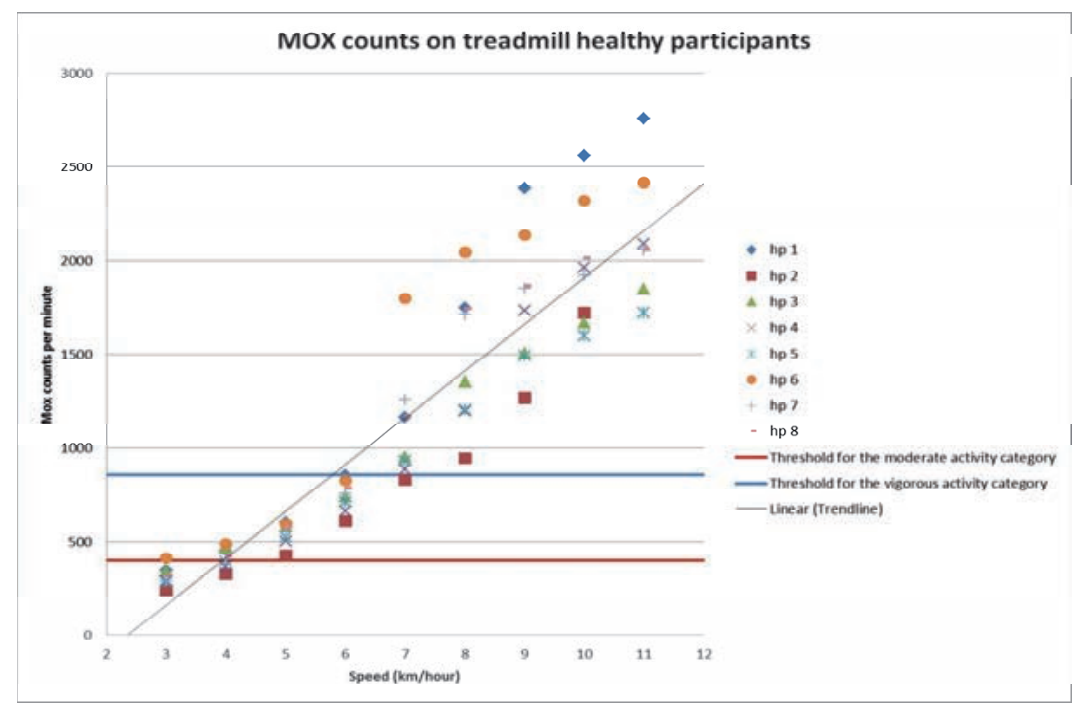

Figure 2 Activity counts per minute of the MOX in healthy participants ( $\mathrm{hp}$ ) during treadmill walking.

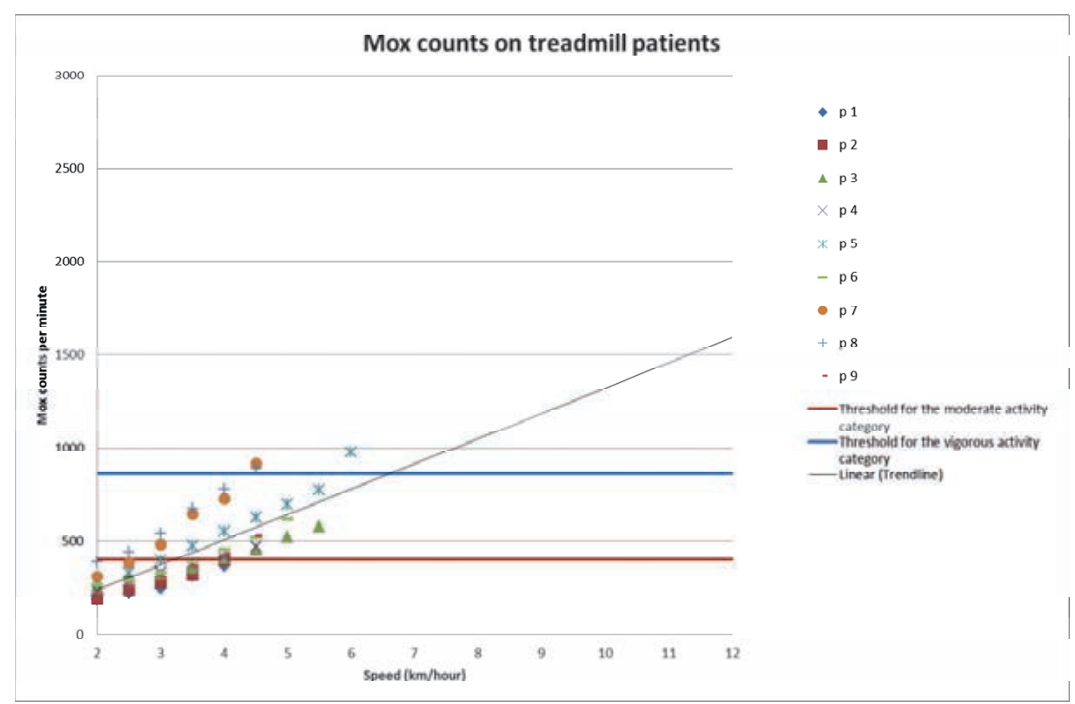

Figure 3 Activity counts per minute of the MOX in patients ( $p$ ) during treadmill walking. 
Daily living

Spearman correlation per day per participant between MOX and ActiGraph counts during daily living was for healthy participants on average $r=0.72$ (range $0.18-0.96$ ), and in patients 0.82 (range $0.60-0.94$ ).

In healthy participants, Bland-Altman plots (Figures 4 and 5) show a mean difference of 9.1 minutes in the moderate category and 1.8 minutes in the vigorous category. The 95\% limits of agreement (LOA) were wide, -37.0 to 18.8 minutes in the moderate category and -10.6 to 7.1 minutes in the vigorous category.

In the patient group, Bland-Altman plots (Figures 6 and 7) showed a mean difference of 2.3 minutes per day in the moderate category between the MOX and the ActiGraph. The limits of agreement were from -27.6 to 22.9 minutes. In the vigorous category LOA ranged from -9.5 to 8.5 , mean difference 0.5 minutes. Within participants there is a consistent over- or underestimation of minutes per category.

In healthy participants the MOX placed 3.6\% of the total minutes in a different category, compared with the ActiGraph; in patients this value was $1.5 \%$. Histograms of counts of the dissimilar classified minutes (Figures 8 and 9) showed that misclassification occurred especially around category thresholds. 


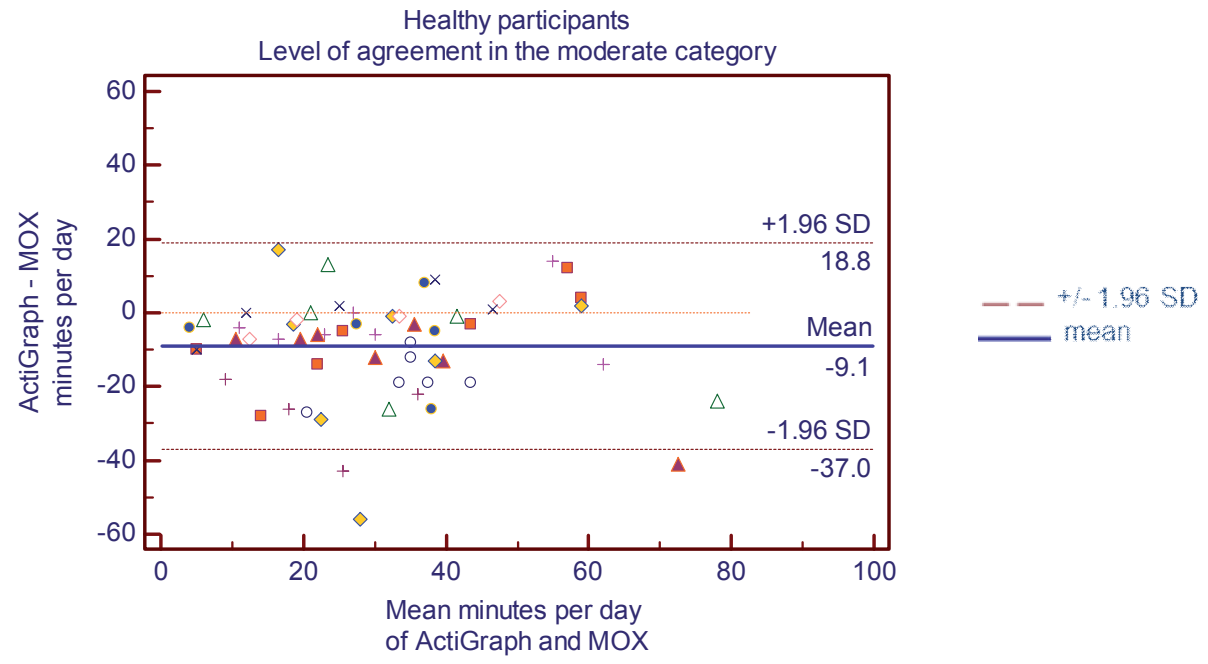

Figure 4 Determination of agreement between ActiGraph and MOX in minutes in the moderate intensity category (for healthy participants) by Bland-Altman plots for repeated measures.

The solid line represents the mean difference between the devices

The dashed lines represent the limits of agreement

Each symbol with its unique colour represents a single participant

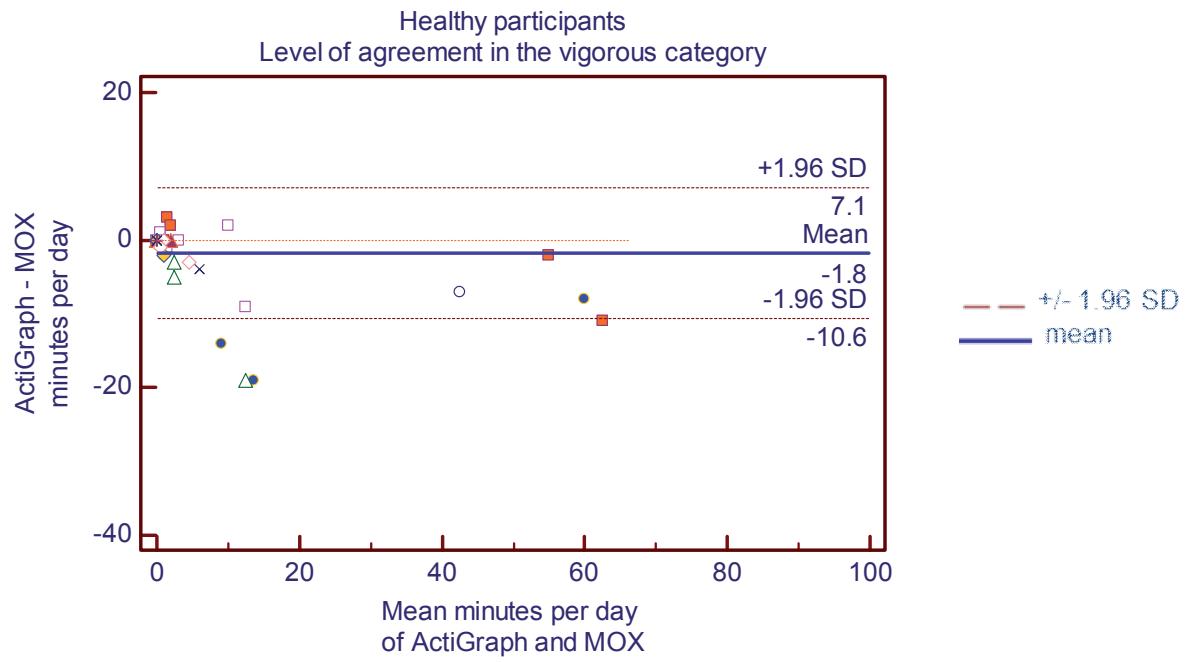

Figure 5 Determination of agreement between ActiGraph and MOX in minutes in the vigorous intensity category (for healthy participants) by Bland-Altman plots for repeated measures.

The solid line represents the mean difference between the devices

The dashed lines represent the limits of agreement

Each symbol with its unique colour represents a single participant 


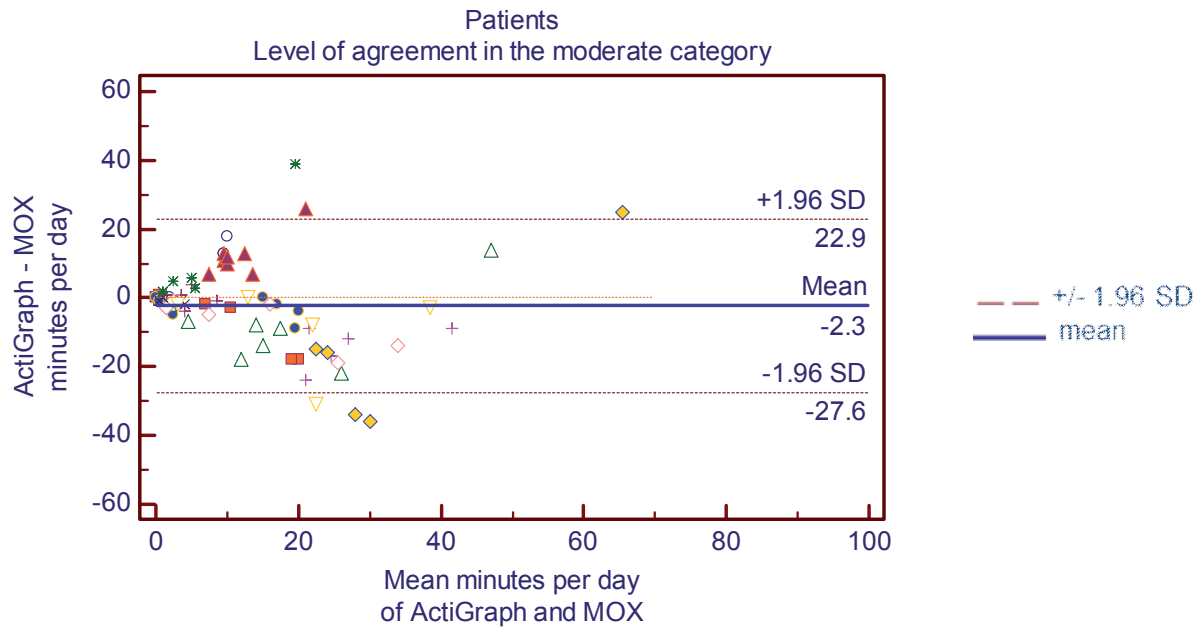

Figure 6 Determination of agreement between ActiGraph and MOX in minutes in the moderate intensity category (for patients) by Bland-Altman plots for repeated measures.

The solid line represents the mean difference between the devices

The dashed lines represent the limits of agreement

Each symbol with its unique colour represents a single participant

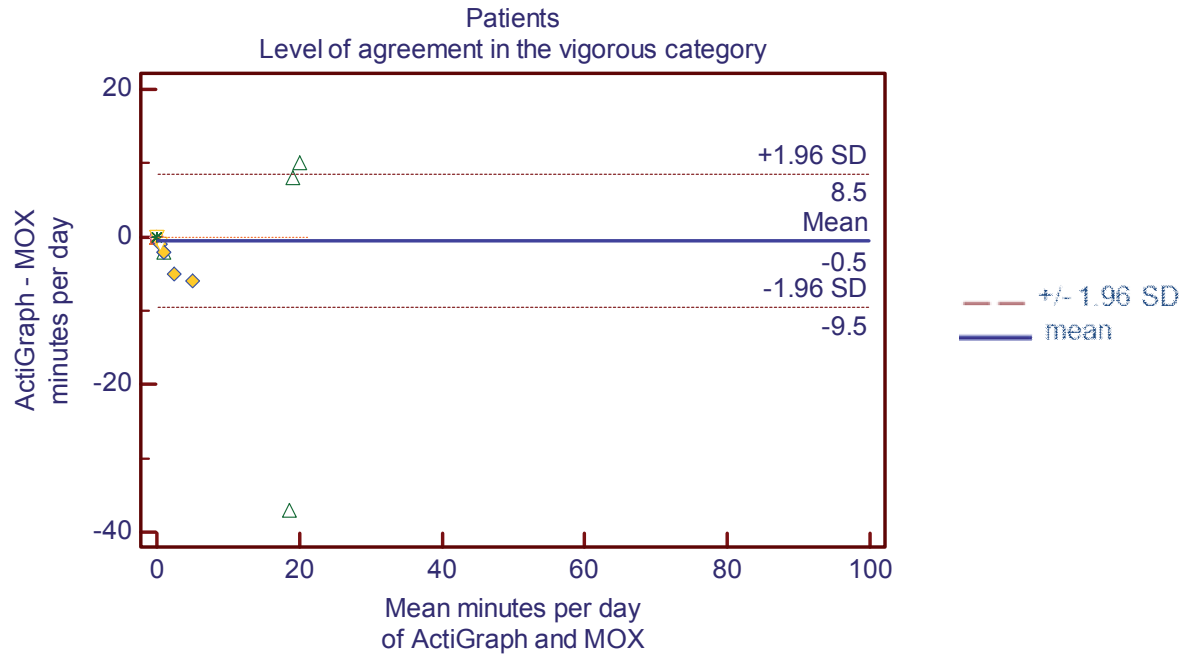

Figure 7 Determination of agreement between ActiGraph and MOX in minutes in the vigorous intensity category (for patients) by Bland-Altman plots for repeated measures. The solid line represents the mean difference between the devices The dashed lines represent the limits of agreement Each symbol with its unique colour represents a single participant 


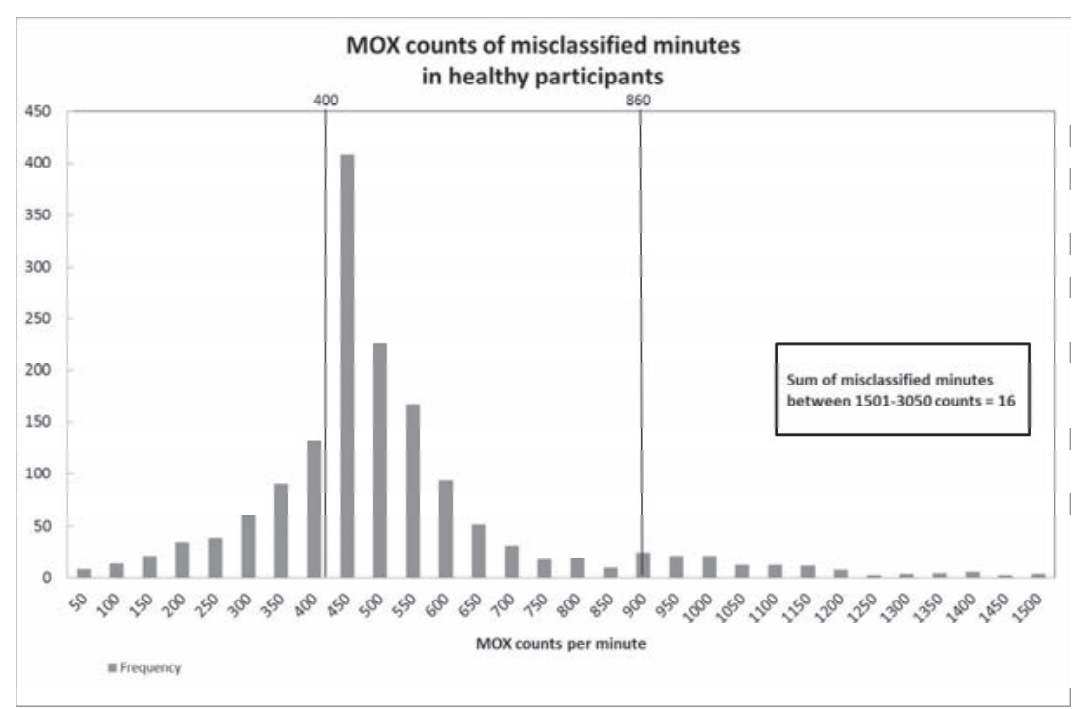

Figure 8 Histogram of counts of misclassified minutes in healthy participants.

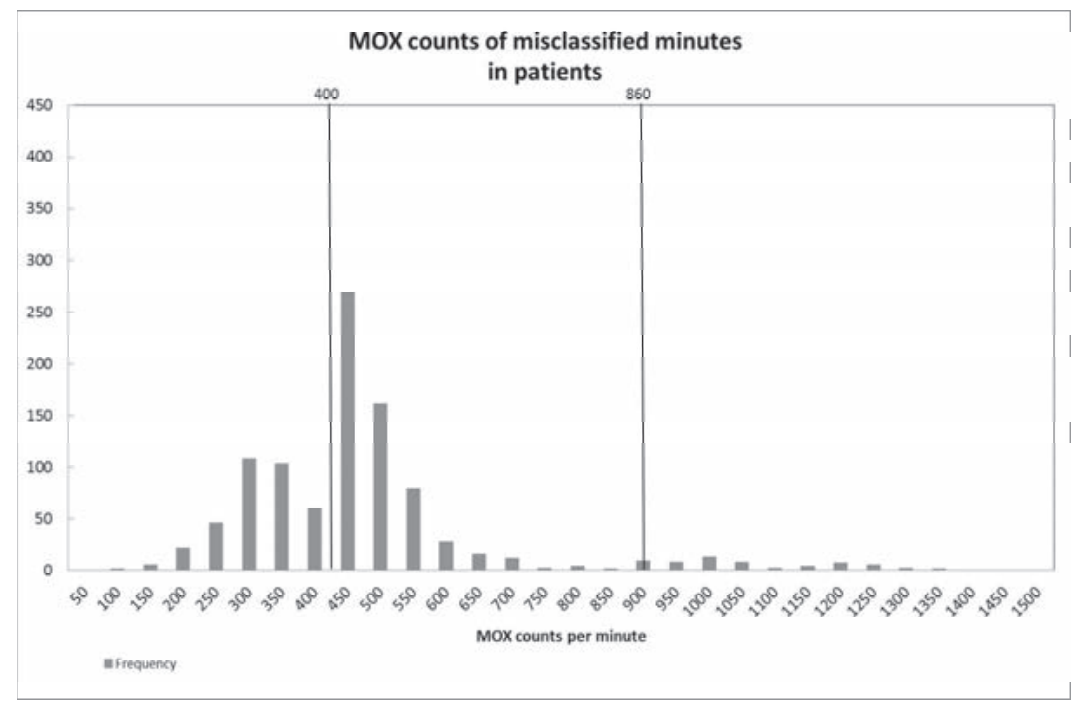

Figure 9 Histogram of counts of misclassified minutes in patients.

\section{Discussion}

In this study thresholds for moderate and vigorous intensity physical activity were defined for the MOX (the activity monitor embedded in the It's LiFe! tool) and the activity 
results from the MOX were compared with the activity results from the ActiGraph GT3X to assess concurrent validity.

MOX activity counts per minute did significantly increase with speed and had an excellent correlation with the reference activity monitor, the ActiGraph GT3X, on the treadmill (mean $r=0.99$ and 0.98 respectively) and a moderate to good correlation in daily living (mean $r=0.72$ and 0.82 respectively). Bland-Altman plots showed that during daily living there was no perfect agreement between the number of minutes the MOX and the ActiGraph placed in the moderate and vigorous intensity activity categories. Sometimes, the MOX overestimated activity compared with the ActiGraph and sometimes the MOX underestimated activity, with an average overestimation in both categories. In total the MOX misclassified $3.6 \%$ of the total analysed minutes of healthy participants and $1.5 \%$ of the minutes for patients. With the chosen design it is not known, however, whether the ActiGraph or the MOX is responsible for the misclassification. The ActiGraph GT3X does not have a perfect correlation ( $r=0.68)$ with measures of energy expenditure, $\mathrm{DLW}^{17}$ or indirect calorimetry $(r=0.77) .{ }^{16}$ In addition, in this study the ActiGraph did not work on 21 days out of 156 days (these days were excluded from the analyses) and sometimes there were suspicious data, i.e. long periods of zero ActiGraph counts were observed on 7 days (included in the analyses). A check of the analyses without these days resulted in minimal changes. Most important, however, was that misclassification did occur around category thresholds, which shows that misclassification is inevitable with the choice of hard thresholds.

A potential alternative for hard thresholds are 'fuzzy logic sets'. With the fuzzy logic classifier a count will not be classified in one activity category but it will represent how much it corresponds to each category. At that moment if-then rules will be applied to classify the count to an activity category. ${ }^{20}$

Another issue with hard thresholds is that at-the-same-speed counts differ between participants, and variance between participants increased with speed. Therefore there is a difference in the prediction equation if running is included (in healthy participants). Another study also proved that the slope of prediction equations differs among different activities. $^{21}$ Also in other studies the variance of the counts increased with speed, ${ }^{22-24}$ even the variance in $\mathrm{VO}_{2}\left(\mathrm{~mL} \cdot \mathrm{kg}^{-1} \cdot \mathrm{min}^{-1}\right)$ increased with speed. ${ }^{25,26}$ However, within participants MOX counts increased consistently and during daily life there was a merely consistent over- or underestimation of activity per participant. The difference in counts per participant could be caused by a different walking pattern, body composition or a slightly different placement of the device. For instance, a small person with a higher step frequency than a taller person will collect more counts per minute. Therefore it is hard to set one threshold for all users. For an equation to calculate personal thresholds at 3 METS, based on weight, height and age, the MOX should be validated with DLW or indirect calorimetry.

However, a different threshold per user is undesirable in daily practice and does not guarantee a perfect fit between threshold and user. In a recent study by Santos-Lozano 
et al. $^{27}$ new equations were formulated to predict energy expenditure from activity counts, age, body mass and gender in different age groups. With the new equations, more accurate thresholds were defined. Yet, significant differences between energy expenditure calculated from the equations and indirect calorimetry were still observed at certain speeds. In daily living differences between energy expenditure measured by accelerometer output and indirect calorimetry will be even higher because accelerometers worn on the hip are mostly sensitive to gait related activities and not for activities involving upper-extremity movement or seated activities. ${ }^{28}$ Furthermore, people with chronic disorders like COPD have a poor mechanical efficiency compared with healthy people, ${ }^{29}$ which means that a threshold set at 3 METS for the general population is not achievable for people with COPD. Consequently results of the It's LiFe! tool will not be a perfect representation of an individual's physical activity. In a previous study ${ }^{30}$, accelerometer output resulted in a $16.4 \%$ difference with direct observation of energy expenditure. Future research could refine MOX thresholds, with a validation study with indirect calorimetry. Also because the thresholds in this study were defined while the MOX and the ActiGraph were placed on the lower back, when in reality they will be worn on the hip, and ActiGraph thresholds were defined for wearing on the hip.

In the It's LiFe! intervention the uncertainties caused by the rigid thresholds are solved by a pre-measurement period. Participants of the intervention use the tool for two weeks and after this period they set a personal goal in collaboration with their care provider. Thus individual factors which influence activity outcome are taken into account and personal progress is measured after goal setting. Furthermore, care providers are instructed to lower the threshold (270 counts per minute for $\pm 2 \mathrm{~km} / \mathrm{h}, 363$ counts for $\pm 3 \mathrm{~km} / \mathrm{h}$ ) in case the pre-measurement reveals that a participant is unable to exceed the general threshold while walking. This does not overcome the fact that non gait related activities will be underestimated. Therefore the participants have the opportunity to write down remarks in the system to notate extra activities.

In conclusion, the MOX is able to assess differences in activity intensity and classify free-living physical activity behaviour in minutes and can be used in the It's LiFe! intervention. However one should bear in mind the general limitations of an accelerometer. One of the strengths of the intervention is that it starts with a personal premeasurement. To estimate energy expenditure with the MOX, the MOX should be validated with DLW or indirect calorimetry. The effects on physical activity of the It's LiFe! tool embedded in primary care practice, will be evaluated in a randomized controlled trial in 24 general practices. 


\section{Acknowledgements}

We would like to thank all participants. The It's LiFe! tool is a co-creation of the researchers, the engineering department of the faculty of Health Medicine and Life Science of Maastricht University (IDEE) and the companies: Maastricht Instruments BV, Maastricht, the Netherlands and Sananet Care BV, Sittard, the Netherlands. The researchers bought the tool from the companies and have no competing financial interests. This study was funded by the Netherlands Organization for Health Research and Development (ZonMw).

\section{References}

1. Murphy SL. Review of physical activity measurement using accelerometers in older adults: considerations for research design and conduct. Preventive medicine. Feb 2009;48(2):108-114.

2. Warburton DE, Nicol CW, Bredin SS. Health benefits of physical activity: the evidence. Canadian medical association journal. 2006;174(6):801-809.

3. World Health Organization. Physical Inactivity: A Global Public Health Problem. Global Strategy on Diet, Physical Activity and Health. 2013 (February 2015). http://www.who.int/dietphysicalactivity/factsheet_inactivity/en/\#.Ug4iwM91cg8.email.

4. Orrow G, Kinmonth A-L, Sanderson S, Sutton S. Effectiveness of physical activity promotion based in primary care: systematic review and meta-analysis of randomised controlled trials. Bmj. 2012-03-26 00:00:00 2012;344.

5. Avery L, Flynn D, van Wersch A, Sniehotta FF, Trenell MI. Changing physical activity behaviour in type 2 diabetes: a systematic review and meta-analysis of behavioural interventions. Diabetes care. Dec 2012;35(12):2681-2689.

6. van Achterberg T, Huisman-de Waal GG, Ketelaar NA, Oostendorp RA, Jacobs JE, Wollersheim HC. How to promote healthy behaviours in patients? An overview of evidence for behaviour change techniques. Health Promot Int. Jun 2011;26(2):148-162.

7. Bravata DM, Smith-Spangler C, Sundaram V, et al. Using pedometers to increase physical activity and improve health: a systematic review. Jama. Nov 21 2007;298(19):2296-2304.

8. Plasqui G, Westerterp KR. Physical activity assessment with accelerometers: an evaluation against doubly labeled water. Obesity. Oct 2007;15(10):2371-2379.

9. van der Weegen S, Verwey R, Spreeuwenberg M, Tange H, van der Weijden T, de Witte L. The Development of a Mobile Monitoring and Feedback Tool to Stimulate Physical Activity of People with a Chronic Disease in Primary Care: A User-Centred Design. JMIR mhealth and uhealth. 2013;1(2):e8.

10. van der Weegen S, Verwey R, Tange HJ, Spreeuwenberg MD, de Witte LP. Usability testing of a monitoring and feedback tool to stimulate physical activity. Patient Prefer Adherence. 2014;8:311-322.

11. Verwey R, van der Weegen S, Spreeuwenberg M, Tange H, van der Weijden T, de Witte L. A pilot study of a tool to stimulate physical activity in patients with COPD or type 2 diabetes in primary care. Journal of Telemedicine and Telecare. Jan 2014;20(1):29-34.

12. Verwey R, van der Weegen S, Tange H, Spreeuwenberg M, van der Weijden T, de Witte L. Get moving: the practice nurse is watching you! A case study of the user-centred design process and testing of a webbased coaching system to stimulate the physical activity of chronically ill patients in primary care. Informatics in primary care. 2012;20(4):289-298.

13. Hanggi JM, Phillips LR, Rowlands AV. Validation of the GT3X ActiGraph in children and comparison with the GT1M ActiGraph. Journal of science and medicine in sport / Sports Medicine Australia. Jan 2013;16(1):4044. 
14. Sasaki JE, John D, Freedson PS. Validation and comparison of ActiGraph activity monitors. Journal of science and medicine in sport / Sports Medicine Australia. Sep 2011;14(5):411-416.

15. Vanhelst J, Mikulovic J, Bui-Xuan G, et al. Comparison of two ActiGraph accelerometer generations in the assessment of physical activity in free living conditions. BMC Res Notes. 2012;5:187.

16. Van Remoortel H, Raste $Y$, Louvaris Z, et al. Validity of six activity monitors in chronic obstructive pulmonary disease: a comparison with indirect calorimetry. PLoS One. 2012;7(6):e39198.

17. Rabinovich RA, Louvaris Z, Raste $Y$, et al. Validity of physical activity monitors during daily life in patients with COPD. Eur Respir J. Feb 82013.

18. Annegarn J, Spruit MA, Savelberg HH, et al. Differences in walking pattern during 6-min walk test between patients with COPD and healthy subjects. PLoS One. 2012;7(5):e37329.

19. Bouten CV, Koekkoek KT, Verduin M, Kodde R, Janssen JD. A triaxial accelerometer and portable data processing unit for the assessment of daily physical activity. Biomedical Engineering, IEEE Transactions on. 1997;44(3):136-147.

20. Preece SJ, Goulermas JY, Kenney LP, Howard D, Meijer K, Crompton R. Activity identification using bodymounted sensors - a review of classification techniques. Physiological measurement. 2009;30(4):R1.

21. van Hees VT, van Lummel RC, Westerterp KR. Estimating activity-related energy expenditure under sedentary conditions using a tri-axial seismic accelerometer. Obesity. Jun 2009;17(6):1287-1292.

22. Esliger DW, Rowlands AV, Hurst TL, Catt M, Murray P, Eston RG. Validation of the GENEA Accelerometer. Med Sci Sports Exerc. Jun 2011;43(6):1085-1093.

23. Dowd KP, Harrington DM, Donnelly AE. Criterion and concurrent validity of the activPAL professional physical activity monitor in adolescent females. PLoS One. 2012;7(10):e47633.

24. Kaminsky LA, Ozemek C. A comparison of the Actigraph GT1M and GT3X accelerometers under standardized and free-living conditions. Physiological measurement. Nov 2012;33(11):1869-1876.

25. Vanhelst J, Hurdiel R, Mikulovic J, et al. Validation of the Vivago Wrist-Worn accelerometer in the assessment of physical activity. BMC Public Health. 2012;12:690.

26. Trost SG, Way R, Okely AD. Predictive validity of three ActiGraph energy expenditure equations for children. Med Sci Sports Exerc. 2006;38(2):380.

27. Santos-Lozano A, Santin-Medeiros F, Cardon G, et al. Actigraph GT3X: Validation and Determination of Physical Activity Intensity Cut Points. Int J Sports Med. May 222013.

28. Hendelman D, Miller K, Baggett C, Debold E, Freedson P. Validity of accelerometry for the assessment of moderate intensity physical activity in the field. Med Sci Sports Exerc. Sep 2000;32(9 Suppl):S442-449.

29. Baarends EM, Schols AM, Pannemans DL, Westerterp KR, Wouters EF. Total free living energy expenditure in patients with severe chronic obstructive pulmonary disease. Am J Respir Crit Care Med. Feb 1997;155(2):549-554.

30. Choquette S, Hamel M, Boissy P. Accelerometer-based wireless body area network to estimate intensity of therapy in post-acute rehabilitation. J Neuroeng Rehabil. 2008;5:20. 



\section{CHAPTER 5 \\ Concurrent validity of the Pam AM300 accelerometer compared to the ActiGraph GT3X}

Submitted

van der Weegen S, Essers H, Spreeuwenberg M, Verwey R, Tange H, de Witte L, Meijer K. Concurrent validity of the Pam AM300 accelerometer compared to the ActiGraph GT3X. 


\section{Abstract}

Objectives Test the validity of the relatively cheap monitoring and feedback system, the Personal Activity Monitor AM300 (Pam), relative to speed and predicted METS (according to the formula of the American College of Sports Medicine) and the ActiGraph GT3X on a treadmill and relative to the ActiGraph, during activities in daily living (ADL).

Study design Concurrent validity study.

Methods Fifteen healthy participants walked on a treadmill (3 to $10 \mathrm{~km} /$ hour) with an ActiGraph and a Pam on the right hip. For ADL measurements, ten healthy volunteers and twelve people with a chronic disease (COPD or type 2 diabetes) wore both devices for 6-7 consecutive days on the lower back.

Results The Pam had a good mean Pearson correlation with speed during walking $(r=0.99)$ and running $(r=0.99)$ in contrast with the ActiGraph which had a low correlation during running $(r=0.43)$. This occurred because ActiGraph counts levelled off at 10 $\mathrm{km} /$ hour in six participants. The mean Spearman correlation of the METS per 15 minutes between the two devices was 0.84 (range 0.43-1.0) in healthy participants and 0.90 (range 0.0-0.98) in patients. Bland-Altman plots showed that in ADL, there was an average agreement between the METS per 15 minutes and a low agreement between the minutes of activity per day between the Pam and the ActiGraph.

Conclusions The relatively inexpensive Pam performs well on the treadmill, in relation to predicted METS and speed. However, the Pam has a low to average agreement with the ActiGraph during daily living, it is not known however if the misclassification occurred due to the Pam or the ActiGraph. 


\section{Introduction}

Physical activity (PA) is an essential element in the primary, secondary and tertiary prevention of several chronic diseases. ${ }^{1,2}$ Therefore, the quantification of physical activity is becoming increasingly important in observational and experimental research, health promotion and disease prevention.

For research purposes, objective measures, such as accelerometry-based activity monitors, are widely used since they provide information on the frequency, amount, duration and intensity of PA, which overcomes several limitations of self-report measures. Commonly used activity monitors, like the ActiGraph, the activPAL ${ }^{\text {TM }}$ and the Actical are relatively expensive. Nowadays, low-cost accelerometers to measure physical activity are rapidly entering the market, such as the Personal Activity Monitor AM300, which is offered at a quarter of the costs. For the promotion of physical activity in the general population and in people with a chronic disease, this is a positive development. Especially because, the use of simple pedometers and accelerometers with a feedback system has shown promising results in accomplishing physical activity behaviour change. ${ }^{3,4-7}$ However, the validity of many low-cost accelerometers on the consumer market is not known.

The Personal Activity Monitor AM300, is a tri-axial accelerometer that, via its display, provides the user with real-time feedback in the form of a Pam score on the summed activity throughout the day. With a wireless connection, additional information about the minutes of activity per day can be viewed on a personal website. If the Pam is a valid instrument, it is a low-cost alternative for objective physical activity measurement and the provision of immediate feedback. The previous version of the Pam, the uni-axial AM101, had a high correlation with indirect calorimetry for treadmill walking $\left(r^{2}=0.92\right)$ and stair walking $\left(r^{2}=0.74\right){ }^{8}$ Theoretically, the tri-axial Pam should be more accurate than the uniaxial Pam, since it measures acceleration in all geometric planes rather than simply in the vertical plane. ${ }^{9}$

Accelerometers can be validated with doubly labelled water (DLW), (video) observation, indirect calorimetry, or comparison to another accurate accelerometer. In this study, the Pam AM300 is validated relative to the ActiGraph GT3X, because this method is suitable in daily living, and DLW compares energy expenditure (EE) rather than activity levels over time.

The ActiGraph GT3X is one of the most widely used and validated activity monitors in research environments. ${ }^{10-12}$ In a study where six activity monitors were evaluated, the ActiGraph had one of the best correlations ( $r=0.77)$ with indirect calorimetry. ${ }^{13}$ Compared with DLW, the ActiGraph showed a correlation of $r=0.68 p<0.0001$ with Activity Energy Expenditure (AEE). ${ }^{14}$ The ActiGraph GT3X is able to explain 53\% of the variance in Total Energy Expenditure (TEE). ${ }^{14}$

The purpose of this study was to test the concurrent validity of the tri-axial Pam AM300 relative to the ActiGraph GT3X, while walking and running on a treadmill and 
during activities of daily living (ADL), both in healthy adults and in people with COPD or type 2 diabetes. Because the ActiGraph is not a real golden standard for physical activity measurement, the metabolic equivalents (METS) values from the Pam and ActiGraph were also compared with treadmill speed and predicted METS estimated by the metabolic equation from the American College of Sports Medicine (ACSM).

\section{Methods}

\section{Participants}

Twenty-five healthy volunteers (18 years and older) and twelve people with COPD $(n=5)$ or type 2 diabetes ( $n=7$ ) (40 years and older), who were able to walk independently, were recruited by an invitation letter from the researchers' local networks, by patient representatives from the national patient associations, and by a practice nurse. Written informed consent was obtained from all participants before study initiation. Participants had the opportunity to withdraw from the study at any time.

\section{Activity monitors}

The Pam (Pam, Pam B.V. Doorwerth, the Netherlands), model AM300, 68×33×10 mm, 20 $\mathrm{g}$, measures accelerations in the range of -4 to $+4 \mathrm{~g}$, in three planes with a sample frequency of $10 \mathrm{~Hz}$ by means of a piezoelectric sensor. Data is transferred to a website by a wireless USB receiver. The summed activity throughout the day is presented on its display screen as a Pam score. On a personal website, minutes of activity per day per activity category (light, 1.8-2.99 METS; moderate, 3-7 METS; and vigorous, >7 METS) can be viewed as well as Pam scores per 15 minutes.

For the current study, the manufacturer made an extra program available that provided METS data per second, which was required for direct comparison with the ActiGraph GT3x. METS data per second was not available for the ADL measurements, since participants should remain within 5 metres of the USB receiver of the computer for this program.

The ActiGraph GT3X (ActiGraph LLC, Pensacola, FL, USA) is a 3-axis activity monitor $(3.8 \mathrm{~cm} \times 3.7 \mathrm{~cm} \times 1.8 \mathrm{~cm})$ that measures accelerations in the range of -3 to $+3 \mathrm{~g}$, with a sample frequency of $30 \mathrm{~Hz}$, which provides activity data in a scale from 0 to 15.000 counts per minute. The counts were recorded in the internal memory housed in the monitor, and transferred to a computer with a micro USB. For the ActiGraph, the program ActiLife v5.10.0 was used to obtain the data; real time feedback from the ActiGraph is not available. 


\section{Data collection procedures}

Treadmill

Fifteen healthy participants walked on a treadmill with an ActiGraph and a Pam on the anterior superior iliac spine (hip). Participants started walking at $3 \mathrm{~km} /$ hour for 2 minutes; subsequently, the speed increased with $1 \mathrm{~km} /$ hour until $10 \mathrm{~km} /$ hour or until the participant indicated that they wanted to stop. Subsequently, length and weight of each participant was measured to the nearest $0.1 \mathrm{~cm} / \mathrm{kg}$ with light clothes and without shoes.

\section{Monitoring of activities during daily living}

Ten healthy volunteers and twelve chronically ill patients were asked to wear the ActiGraph and the Pam with an elastic belt on their lower back for 6-7 consecutive days, during waking hours. Participants performed their normal daily life activities and reported their wear time in a diary. ActiGraph-data of ADL measurements were also used in another study ${ }^{15}$.

\section{Data processing}

\section{Treadmill}

During the treadmill activity, measurements of metabolic equivalents (METS) per second from the Pam were obtained, and average METS per minute were calculated per speed. The ActiGraph counts per minute were converted to average METS per minute per speed with the formula from Sasaki: ${ }^{12}$

METS calculated by ActiGraph data= 0.000863 (Vector Magnitude Unit) + 0.668876

MET output by the devices was compared to METS calculated using the formula of the American College of Sports Medicine (ACSM). ${ }^{16}$

METS calculated by walking speed $=$

$(0.1$ (speed) +1.8 (speed) (fractional grade) +3.5$) / 3.5$

METS calculated by running speed $=$

$$
(0.2 \text { (speed) }+0.9 \text { (speed) (fractional grade) }+3.5) / 3.5
$$

Speed $=$ speed in meters $x \mathrm{~min}^{-1}$, fractional grade was $0 \%$

Daily living

From ADL measurements, the Pam database provides the average Pam score per 15 minutes. 
The Pam score was converted into MET values per 15 minutes by the following formula, which was provided by the manufacturer:

\section{METS calculated by Pam data= (1440/1500) (Pam score) +1}

In addition, the Pam provides minutes per day spent in each of the following categories: light (1.8-3 METS), moderate (3-7 METS) and vigorous (>7 METS). Since ActiGraph thresholds are based on the moderate category (3-6 METS), analyses were done on the combined category of moderate to vigorous activities ( $\geq 3$ METS).

The ActiGraph GT3X provides activity counts per minute as a composite vector magnitude of the three axes (VM3). ${ }^{12}$ The counts per 15 minutes were averaged, and the average METS per 15 minutes were calculated using the abovementioned formula from Sasaki's article. ${ }^{12}$ To determine in which activity category one was active, thresholds from the same article were used.

\section{Data analysis}

All analyses were performed using IBM Statistical Product and Service Solutions (SPSS version 19.0). Graphs were drawn in Microsoft Excel 2010, and MedCalc version 12.5.0.0 was used to conduct Bland-Altman plots for repeated measures. Outcome measures were METS per minute on the treadmill and during daily living; METS per 15 minutes and minutes per day.

\section{Treadmill}

Data was analysed for walking (3-6 km/hour) and running (8-10 km/hour) separately, since these are different types of locomotion resulting in different slopes in the relation between speed and METS. The speed of $7 \mathrm{~km} /$ hour was left out of the analysis, since at this speed some participants were running, while others were still walking.

Pearson correlation was calculated per person to compare METS from both devices with treadmill speed and to compare the METS from the Pam with the METS from the ActiGraph. In addition, the agreement of the MET output of the devices with the predicted MET values (by the MET equation from the American College of Sports Medicine) was examined in scatterplots, the absolute difference per participant at each speed was calculated and paired t-test were conducted to compare the absolute differences with the predicted METS between the ActiGraph and the Pam. Because there were multiple measurements per person, a multilevel linear mixed model analysis was applied. This was done with speed at level 1 and participant at level 2. 


\section{Daily living}

Spearman correlations between the METS per 15 minutes of the ActiGraph and the Pam were calculated per person per day. Inter-monitor agreement for METS per 15 minutes and minutes of physical activity ( $\geq 3$ METS) per day were examined using Bland-Altman plots for repeated measures. To prevent a high correlation between the devices because of non-wear time, these periods were omitted from analyses, based on diary information from the participants. Correlations over 0.8 were rated as good.

\section{Results}

Physical characteristics of the participants are listed in Table 1. Of the twenty-two participants who participated in the daily living measurements, 5 people were diagnosed with COPD and 7 people with diabetes type 2 .

Table 1 Physical characteristics of the participants

\begin{tabular}{llll}
\hline & $\begin{array}{l}\text { Treadmill } \\
\text { Healthy }(\mathrm{n}=15)\end{array}$ & $\begin{array}{l}\text { Daily living } \\
\text { Healthy }(\mathrm{n}=10)\end{array}$ & Patients $(\mathrm{n}=12)$ \\
\hline Age (years) & $35.9 \pm 19.6$ & $30.4 \pm 8.3$ & $61.6 \pm 9.2$ \\
Height $(\mathrm{cm})$ & $169.5 \pm 11.8$ & $175 \pm 9.6$ & $168.6 \pm 9.9$ \\
Weight $(\mathrm{kg})$ & $70.4 \pm 13.2$ & $68.1 \pm 8.9(\mathrm{n}=9)$ & $95.6 \pm 31.5$ \\
BMI $(\mathrm{kg} / \mathrm{m} 2)$ & $24.5 \pm 4.0$ & $22.7 \pm 2.8$ & $33.3 \pm 9.8$ \\
FEV1 $(\%$ predicted) & - & - & $50.5 \pm 12.6(\mathrm{n}=4)$ \\
Hba1c $(\mathrm{mmol} / \mathrm{mol})$ & - & - & $53.1 \pm 6.4(\mathrm{n}=7)$ \\
\hline
\end{tabular}

FEV Forced expiratory volume

BMI Body Mass Index

\section{Treadmill}

During treadmill walking, the average correlation per person of the Pam with speed was high 0.99 (range 0.98-1) as well as the correlation between the ActiGraph and speed 1.00 (range 0.97-1). In addition the Pam had a high correlation with the ActiGraph 0.99 (range 0.97-1). During treadmill running, the Pam was still highly correlated with speed 0.99 (range 0.93-1); however, the ActiGraph had a low average correlation per person 0.43 (range -1.00-1). Consequently, Pearson correlations between the Pam and the ActiGraph were low as well 0.49 (range -0.83-1). Visual observation of the scatterplots revealed that in the Pam, METS did increase with speed in all participants. In six participants METS/counts of the ActiGraph were lower at $10 \mathrm{~km} / \mathrm{hour}$ than at 8 or $9 \mathrm{~km} /$ hour.

Figure 1 shows the METS of the ActiGraph and the Pam in each participant and the predicted METS at each speed. ${ }^{16}$ During walking the variance in METS between participants was smaller in the Pam compared to the ActiGraph and the Pam corresponds best with the predicted METS. The absolute difference with the predicted METS was signifi- 
cantly lower in the Pam (mean=0.22 METS, SD=0.15) than in the ActiGraph (mean=0.54 METS, SD=0.44); $t(59)=5.64, p<0.001$. Also during running the absolute difference of the Pam with the predicted METS was significantly lower (mean=1.96 METS, SD=1.54) compared to the ActiGraph (mean=2.82 METS, SD=1.47); $t(40)=2.76, p=0.009$. The ActiGraph underestimates MET-values in all participants during running and the Pam, under estimates MET values in some participants and overestimates MET values in others.

The linear mixed model revealed that METS of the Pam did increase significantly with speed during both walking and running (both $p<0.001$ ).
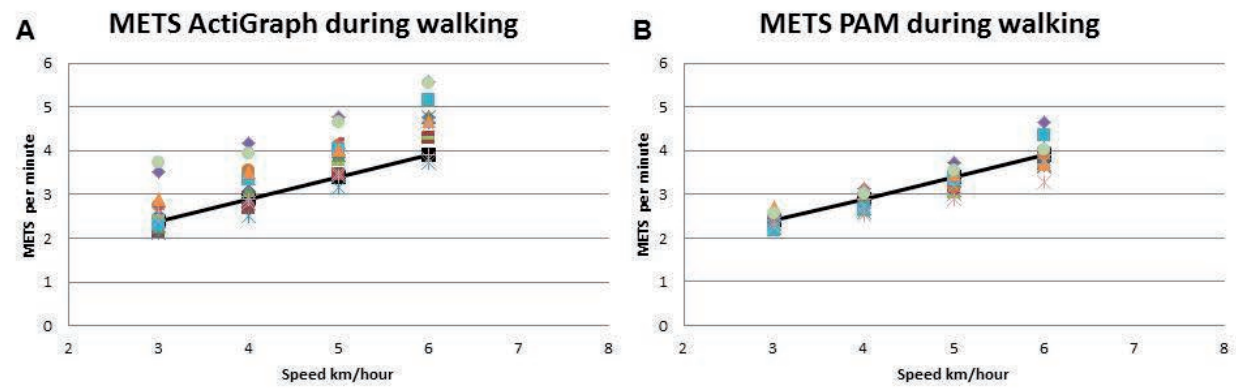

\section{METS ActiGraph during running}

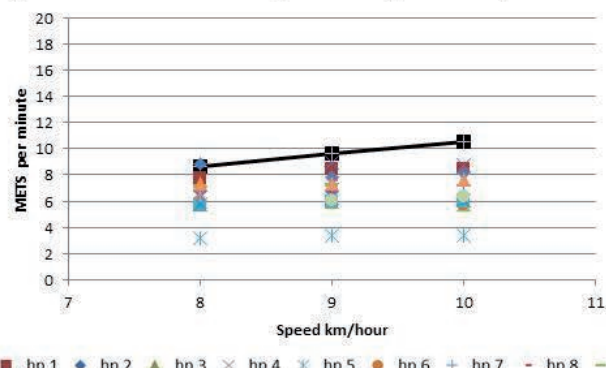

Figure 2 METS of the ActiGraph and the Pam for each healthy participant (hp) and the predicted METS (ACSM) for walking ( $A$ and $B$ ) and running $(C$ and $D)$.

\section{Daily living}

During monitoring of activities in daily living, data transfer discrepancies occurred in both devices. All Pam scores per day were compared to the summed 15-minute Pam scores per day, to detect errors. The manufacturer built a tool to reveal these discrepancies. Fifteen-minute data of the Pam of $25 \%$ of the days was not transferred to the server. The ActiGraph did not record data (<1 MET during the whole day) on $15 \%$ of the days. 
The mean Spearman correlation of the METS per 15 minutes between the two devices was 0.84 (range 0.43-1.0). In patients, the mean spearman correlation was 0.90 (range 0.0-0.98). Low correlations occurred mainly in one healthy participant.

The inter-monitor agreement for METS per 15 minutes is shown in Figure $2 a$ and $2 b$. The mean difference between the devices was 0.3 METS in healthy participants as well as in patients. In healthy participants, the mean difference in minutes per day in the moderate to vigorous category was 16.1 minutes. The 95\% limits of agreement (LOA) were wide, from -43.2 to 11.0 minutes (Figure $2 \mathrm{c}$ ). The mean difference between the ActiGraph and the Pam in patients was 12.5 minutes per day in the moderate to vigorous category. LOA ranged from -29.4 to 4.5 minutes per day (Figure $2 \mathrm{~d}$ ).

A

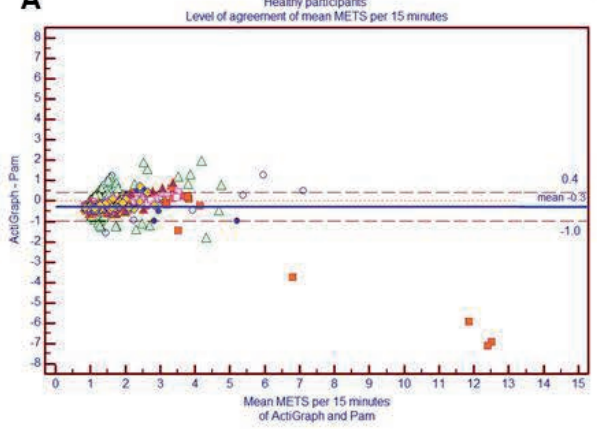

C

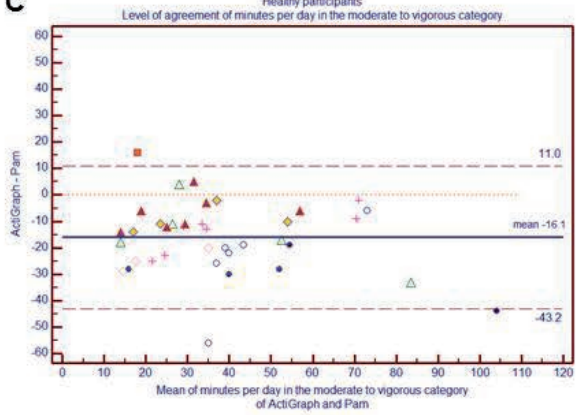

B

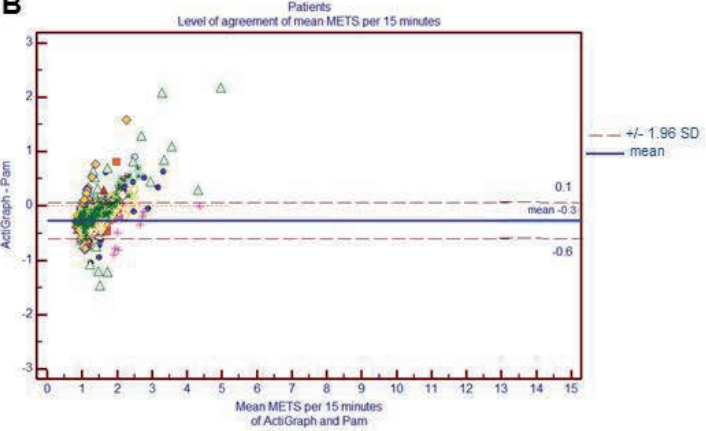

D

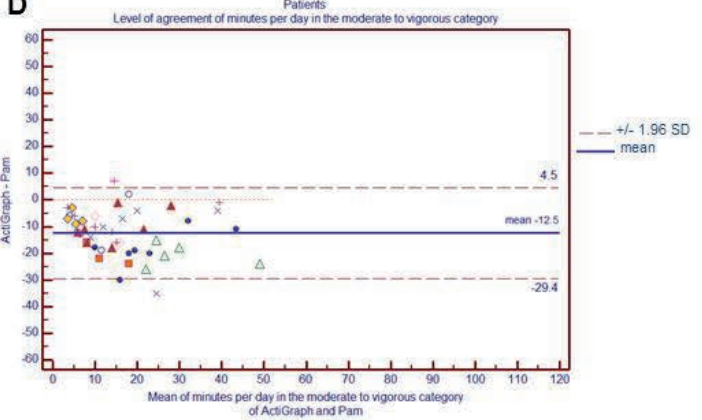

Figure 2 Agreement between ActiGraph and Pam on the back in healthy participants ( $A$ and $C$ ) and patients ( $B$ and $D$ ), in METS per 15 minutes ( $A$ and $B$ ) and in minutes per day ( $C$ and $D)$, by Bland-Altman plots for repeated measures per person.

Each symbol with its unique colour represents a single participant 


\section{Discussion}

This study examined the validity of the Pam AM300 relative to speed, the predicted METS (according to the ACSM metabolic equation) and to the ActiGraph GT3X on a treadmill, and to the ActiGraph during daily living. During treadmill walking and running, compared to the ActiGraph the METS measured by the Pam corresponded better with the MET equation from the American College of Sports Medicine. The ActiGraph underestimates MET values during running. In addition, the Pam had a high correlation with speed during walking and running and the ActiGraph only during walking. In both devices, the variance in METS between participants increased with speed. During daily living activities, there was a good correlation (rho $=0.84$ in healthy participants and 0.90 in patients) between the METS per 15 minutes of the Pam and the ActiGraph. BlandAltman plots showed relatively narrow limits of agreement of METS per 15 minutes between the two devices. However, the agreement between the minutes per day in the category of moderate to vigorous ( $\geq 3$ METS) was poor.

The increase in variance of METS, activity counts and $\mathrm{VO}_{2}\left(\mathrm{~mL} \cdot \mathrm{kg}^{-1} \cdot \mathrm{min}^{-1}\right)$ with speed is also observed in other studies. ${ }^{17-21}$ The ActiGraph had a low correlation with speed $(r=0.43)$ during running, even negative correlations were observed, because in six participants the counts were lower at $10 \mathrm{~km} /$ hour than at 8 or $9 \mathrm{~km} /$ hour. In the literature, the levelling-off effect of the ActiGraph is known as the 'plateau phenomenon'. From $10-12 \mathrm{~km} / \mathrm{h}$, there is an inverse curvilinear relationship between exercise intensity and activity counts. This is due to limitations in the ActiGraph's frequency filtering characteristics. $^{22-24}$ Because of a band-pass filter in the ActiGraph, these monitors detect accelerations with frequencies between 0.25 and $2.5 \mathrm{~Hz}$, while the frequency content of acceleration during running at faster speeds easily exceeds $2.5 \mathrm{~Hz}^{22}$ Because of the plateau effect in the ActiGraph, Pearson correlations between the Pam and the ActiGraph in this study were poor during running. Also the three outliers, in the daily living comparison between the METS per 15 minutes of the ActiGraph and the Pam, at 11-13 METS might be caused by the plateau effect of the ActiGraph. In the minutes per day comparison, the Pam overestimated the minutes per day classified in the category of moderate to vigorous, compared to the ActiGraph. With this study design, however, it is not known whether the ActiGraph or the Pam is responsible for misclassification. The misclassification can partly be explained by the MET formula of both devices. The formula of the Pam ends with +1 , while the formula of the ActiGraph ends with $+0.67,{ }^{12}$ which caused an overestimation of 0.23 METS in the Pam compared to the ActiGraph at rest. The Pam formula seems more correct, since the metabolic equivalent at rest is by definition $1 .{ }^{25}$ Another fact that could have caused this difference is that the ActiGraph showed periods of zero counts during the day. In another validation study with the ActiGraph as a reference, the minutes per day do not agree perfectly either. ${ }^{26}$ This occurred because data points cluster around category thresholds, which could be the cause of a difference in minutes per category between the devices in this study as well. If a hard 
threshold (for example activity above 3 METS) is chosen in a study as an outcome measure, this may cause misinterpretation of the data if participants increase their level of activity but do not exceed this threshold. ${ }^{27}$ This could be avoided by presenting total activity rather than threshold-dependent classified data. With the interpretation of accelerometer data, other general limitations of accelerometers should be taken into account, such as the underestimation of activity because of the ignorance of upper body movements, walking on a gradient, and weight bearing activities. ${ }^{28,29}$ However, accelerometers are a relatively good instrument for assessing activity levels in daily living since it is objective, practical and non-invasive. ${ }^{30,31}$

During the daily living period, both the Pam and the ActiGraph failed to record or transfer the data of some days. If an accelerometer is used as an outcome measure, the lack of data could unfairly be understood as sedentary behaviour. Therefore, an additional wear-time diary or data check is necessary. A data check for the Pam is currently available.

Limitations of this study are the failure of data transmission, the small sample size and the comparison of the devices worn on the back in daily living. While wearing on the hip or the back should not differ because both are located around the centre of mass, some researchers recommend wearing the devices on the hip and thresholds are developed while placed on the hip.

\section{Conclusions}

In conclusion, the relatively inexpensive, commercially available Pam worn on the hip can be used for the objective measurement of walking and running. However, at higher speeds the variance and the discrepancy with the ActiGraph increase. With this study design it is not known if the discrepancy is caused by the ActiGraph or the Pam. Future research should validate the Pam, by comparing it with indirect calorimetry or direct observation.

\section{Acknowledgements}

We would like to thank all participants. This study was part of the It's LiFe! project which is funded by ZonMw (The Netherlands Organisation for Health Research and development) in the programme 'New Instruments for Healthcare'. Project number 300040005. We acknowledge Erik Damen (Pam BV, the Netherlands) for providing us with additional software to gather Pam data per minute during treadmill measurements and software to check data transfer discrepancies.

Competing interests: None declared. 
Ethical approval: The Maastricht Medical Research Ethics Committee has reviewed the research protocol, information letters and informed consents and rated that this study was not covered by the medical ethical law and therefore waived ethical approval (registration number 11-4-120).

\section{References}

1. Blair SN, Brodney S. Effects of physical inactivity and obesity on morbidity and mortality: current evidence and research issues. Med Sci Sports Exerc. Nov 1999;31(11 Suppl):S646-662.

2. Warburton DE, Nicol CW, Bredin SS. Health benefits of physical activity: the evidence. Cmaj. Mar 14 2006;174(6):801-809.

3. McKay J, Wright A, Lowry R, Steele K, Ryde G, Mutrie N. Walking on prescription: the utility of a pedometer pack for increasing physical activity in primary care. Patient education and counselling. Jul 2009;76(1):7176.

4. Tudor-Locke C, Lutes L. Why do pedometers work?: a reflection upon the factors related to successfully increasing physical activity. Sports Med. 2009;39(12):981-993.

5. Bravata DM, Smith-Spangler C, Sundaram V, et al. Using pedometers to increase physical activity and improve health: a systematic review. Jama. Nov 21 2007;298(19):2296-2304.

6. Richardson CR, Newton TL, Abraham JJ, Sen A, Jimbo M, Swartz AM. A meta-analysis of pedometer-based walking interventions and weight loss. Ann Fam Med. Jan-Feb 2008;6(1):69-77.

7. Verwey R, van der Weegen S, Spreeuwenberg M, Tange $H$, van der Weijden T, de Witte L. A pilot study of a tool to stimulate physical activity in patients with COPD or type 2 diabetes in primary care. Journal of Telemedicine and Telecare. Jan 2014;20(1):29-34.

8. Slootmaker SM, Chin APMJ, Schuit AJ, van Mechelen W, Koppes LL. Concurrent validity of the Pam accelerometer relative to the MTI Actigraph using oxygen consumption as a reference. Scandinavian journal of medicine \& science in sports. Feb 2009;19(1):36-43.

9. Shumaker SA, Ockene JK, Riekert KA. The handbook of health behaviour change. New York: Springer Pub. Co.; 2009.

10. Hanggi JM, Phillips LR, Rowlands AV. Validation of the GT3X ActiGraph in children and comparison with the GT1M ActiGraph. Journal of science and medicine in sport / Sports Medicine Australia. Jan 2013;16(1):4044.

11. Vanhelst J, Mikulovic J, Bui-Xuan G, et al. Comparison of two ActiGraph accelerometer generations in the assessment of physical activity in free living conditions. BMC Res Notes. 2012;5:187.

12. Sasaki JE, John D, Freedson PS. Validation and comparison of ActiGraph activity monitors. Journal of science and medicine in sport / Sports Medicine Australia. Sep 2011;14(5):411-416.

13. Van Remoortel H, Raste $Y$, Louvaris Z, et al. Validity of six activity monitors in chronic obstructive pulmonary disease: a comparison with indirect calorimetry. PLoS One. 2012;7(6):e39198.

14. Rabinovich RA, Louvaris Z, Raste $Y$, et al. Validity of physical activity monitors during daily life in patients with COPD. Eur Respir J. Feb 82013.

15. van der Weegen S, Essers H, Spreeuwenberg M, et al. Concurrent Validity of the MOX Activity Monitor Compared to the ActiGraph GT3X. Telemed J E Health. Apr 2015;21(4):259-266.

16. Glass S, Dwyer GB. ACSM'S metabolic calculations handbook. Philadelphia; [Indianapolis]: Lippincott Williams \& Wilkins; American College of Sports Medicine; 2007.

17. Esliger DW, Rowlands AV, Hurst TL, Catt M, Murray P, Eston RG. Validation of the GENEA Accelerometer. Med Sci Sports Exerc. Jun 2011;43(6):1085-1093.

18. Dowd KP, Harrington DM, Donnelly AE. Criterion and concurrent validity of the activPAL professional physical activity monitor in adolescent females. PLoS One. 2012;7(10):e47633. 
19. Kaminsky LA, Ozemek C. A comparison of the Actigraph GT1M and GT3X accelerometers under standardized and free-living conditions. Physiological measurement. Nov 2012;33(11):1869-1876.

20. Vanhelst J, Hurdiel R, Mikulovic J, et al. Validation of the Vivago Wrist-Worn accelerometer in the assessment of physical activity. BMC Public Health. 2012;12:690.

21. Trost SG, Way R, Okely AD. Predictive validity of three ActiGraph energy expenditure equations for children. Med Sci Sports Exerc. 2006;38(2):380.

22. John D, Miller R, Kozey-Keadle S, Caldwell G, Freedson P. Biomechanical examination of the 'plateau phenomenon' in ActiGraph vertical activity counts. Physiological measurement. Feb 2012;33(2):219-230.

23. Rowlands AV, Stone MR, Eston RG. Influence of speed and step frequency during walking and running on motion sensor output. Med Sci Sports Exerc. Apr 2007;39(4):716-727.

24. Fudge BW, Wilson J, Easton C, et al. Estimation of oxygen uptake during fast running using accelerometry and heart rate. Med Sci Sports Exerc. Jan 2007;39(1):192-198.

25. McArdle WD, Katch FI, Katch VL. Exercise physiology : energy, nutrition, and human performance. Philadelphia, Pa.; London: Lippincott Williams \& Wilkins; 2006.

26. van der Weegen S, Essers H, Spreeuwenberg M, et al. Concurrent Validity of the MOX Activity Monitor Compared to the ActiGraph GT3X. Telemedicine journal and e-health : the official journal of the American Telemedicine Association. Jan 232015.

27. Bailey DP, Boddy LM, Savory LA, Denton SJ, Kerr CJ. Choice of activity-intensity classification thresholds impacts upon accelerometer-assessed physical activity-health relationships in children. PLoS One. 2013;8(2):e57101.

28. Trost SG, Mclver KL, Pate RR. Conducting accelerometer-based activity assessments in field-based research. Med Sci Sports Exerc. Nov 2005;37(11 Suppl):S531-543.

29. Leenders NY, Sherman WM, Nagaraja HN. Energy expenditure estimated by accelerometry and doubly labeled water: do they agree? Med Sci Sports Exerc. Dec 2006;38(12):2165-2172.

30. Casaburi R. Activity monitoring in assessing activities of daily living. COPD. Sep 2007;4(3):251-255.

31. Hills AP, Mokhtar N, Byrne NM. Assessment of physical activity and energy expenditure: an overview of objective measures. Frontiers in Nutrition. 2014-June-16 2014;1. 



\section{CHAPTER 6}

A monitoring and feedback tool embedded in a counselling protocol to increase physical activity of patients with COPD or type 2 diabetes in primary care: study protocol of a three-arm cluster randomised

This chapter was published as:

Verwey R, van der Weegen S, Spreeuwenberg M, Tange $H$, van der Weijden $T$, de Witte L. A monitoring and feedback tool embedded in a counselling protocol to increase physical activity of patients with COPD or type 2 diabetes in primary care: study protocol of a three-arm cluster randomised controlled trial. BMC Fam Pract. 2014;15(1):93 


\section{Abstract}

Background Physical activity is important for a healthy lifestyle. Although physical activity can delay complications and decrease the burden of the disease, the level of activity of patients with chronic obstructive pulmonary disease (COPD) or type 2 Diabetes Mellitus (DM2) is often far from optimal. To stimulate physical activity, a monitoring and feedback tool, consisting of an accelerometer linked to a smartphone and webserver (It's LiFe! tool), and a counselling protocol for practice nurses in primary care was developed (the Self-management Support Programme). The main objective of this study is to measure the longitudinal effects of this counselling protocol and the surplus of using the tool.

Methods/Design This three-armed cluster randomised controlled trial with 120 participants with COPD and 120 participants with DM2 (aged 40-70), compares the counselling protocol with and without the use of the tool (group 1 and 2) with usual care (group 3). Recruitment takes place at GP practices in the southern regions of the Netherlands. Randomisation takes place at the practice level. The intended sample (three arms of 8 practices) powers the study to detect a 10-minute difference of moderate and intense physical activity per day between groups 1 and 3. Participants in the intervention groups have to visit the practice nurse 3-4 times for physical activity counselling, in a 6-month period. Specific activity goals tailored to the individual patient's preferences and needs will be set. In addition, participants in group 1 will be instructed to use the tool in daily life. The primary outcome, physical activity, will be measured in all groups with a physical activity monitor (Pam). Secondary outcomes are quality of life, general - and exercise - self-efficacy, and health status. Follow-up will take place after 6 and 9 months. Separately, a process evaluation will be conducted to explore reasons for trial non-participation and the intervention's acceptability for participating patients and nurses.

Discussion Results of this study will give insight into the effects of the It's LiFe! monitoring and feedback tool combined with care from a practice nurse for people with COPD or DM2 on physical activity. 


\section{Background}

Because increased physical activity (PA) has positive effects on prognosis and quality of life, ${ }^{1,2}$ stimulating PA is an important element in the treatment of people with chronic diseases such as chronic obstructive pulmonary disease (COPD) or type II diabetes $(\mathrm{DM} 2)^{3,4}$ It is, however, a challenge to adhere to guidelines for healthy exercise (at least 30 minutes of moderate activity five days a week). ${ }^{5,6}$ By integrating PA counselling into routine practice, primary care providers can support patients in meeting this challenge. ${ }^{5,7}$ In the Netherlands the majority of chronically ill patients visit the family practice regularly to monitor their condition, and it is the task of the practice nurse (PN) to provide lifestyle counselling during those consultations. ${ }^{8,9}$

The most common method of PA promotion is verbal advise, followed by print- and computer-based interventions. ${ }^{10}$ Interventions incorporating technology that is readily accessible on a daily basis for monitoring activity levels, such as computers or mobile phones, can support care providers to coach patients in establishing behavioural changes. ${ }^{11}$ Those interventions may facilitate long-term follow-up, ${ }^{12,13}$ and may be an effective way to provide PA counselling without increasing the time demands on primary care providers. ${ }^{14}$

PA counselling has the potential to increase PA levels in the short term. ${ }^{13}$ However, evidence regarding which methods of exercise promotion works best in the long term is still limited. ${ }^{15}$ Furthermore, computer-based patient self-management programs, delivered in health-supported settings, show the potential for changing health behaviours and improving clinical outcomes, but more well designed trials are warranted to test their effectiveness. ${ }^{16}$ Those trials should especially focus on the effects of theorybased intervention development, combined with the effect of tailored advise and feedback. $^{17}$

We therefore, developed and tested a monitoring and feedback tool called It's LiFe! $^{18,19}$ and a corresponding counselling programme for primary care nurses (the Selfmanagement Support Programme). The basic ideas behind this combination are: providing an objective measurement of PA via an accelerometer, collaborative goal setting and automatic feedback via an application on a smartphone combined with PA counselling by the PN. Results from a feasibility study showed that participants were positive about the tool. Regarding the effects of using the tool, a positive trend was seen: the mean level of PA increased by more than 10 minutes per day and patients reported a higher quality of life. ${ }^{20}$

This paper describes the study protocol of a three-armed cluster randomised controlled trial with 120 participants with COPD and 120 participants with DM2 (aged 40-70), comparing the Self-management Support Programme with and without the use of the tool (group 1 and 2) with usual care (group 3). 


\section{Objectives and hypotheses}

The objective of this randomised controlled trial is to evaluate the longitudinal effects of the It's LiFe! tool embedded in a Self-management Support Programme (SSP) on 40-70 years old patients with COPD and DM2 in primary care. The primary outcome measure is PA in daily life. Secondary outcome measures are self-efficacy, quality of life and health status. The main difference that is evaluated is between the whole intervention and usual care. Additionally, the added value of the tool is evaluated. Apart from the effect evaluation, a process evaluation will be performed, aimed at getting insight into the adherence to the intervention and the acceptance of the intervention by participating patients and PNs.

The main hypothesis is that the whole intervention will increase PA on a moderate level by at least 10 minutes per day, over a six-month period, and to maintain this increase over three months.

\section{Methods/design}

This paper was written according to the CONSORT 2010 statement: extension to cluster randomised trials. ${ }^{21}$

\section{Study design}

The study is designed as a cluster randomised controlled trial with GP practices as the unit of randomisation. To compare the whole intervention with both usual care and SSP only (to isolate the effect of the tool), the trial has three arms: the use of a monitoring and feedback tool embedded in the SSP (group 1), the SSP without the tool (group 2), and usual care (group 3). The CONSORT flowchart (Figure 1) summarises the trial design. The population consists of 120 participants with COPD and 120 participants with DM2 from 24 GP practices. Each practice provides 5 COPD patients and 5 DM2 patients, which makes a total of 40 patients with COPD and 40 patients with DM2 from 8 practices per trial arm.

\section{Eligibility}

Participants between 40 and 70 years old are eligible when they are diagnosed with COPD or DM2, are treated in primary care, and in the opinion of the PN, do not comply with the Dutch Norm for Healthy Exercise. 6 Additional inclusion criteria for the DM2 patients are a BMI > 25 and for the COPD patients: a clinical diagnosis of COPD according to the GOLD-criteria stage $1-3$, being at least six weeks respiratory stable and on a stable drug regimen. 


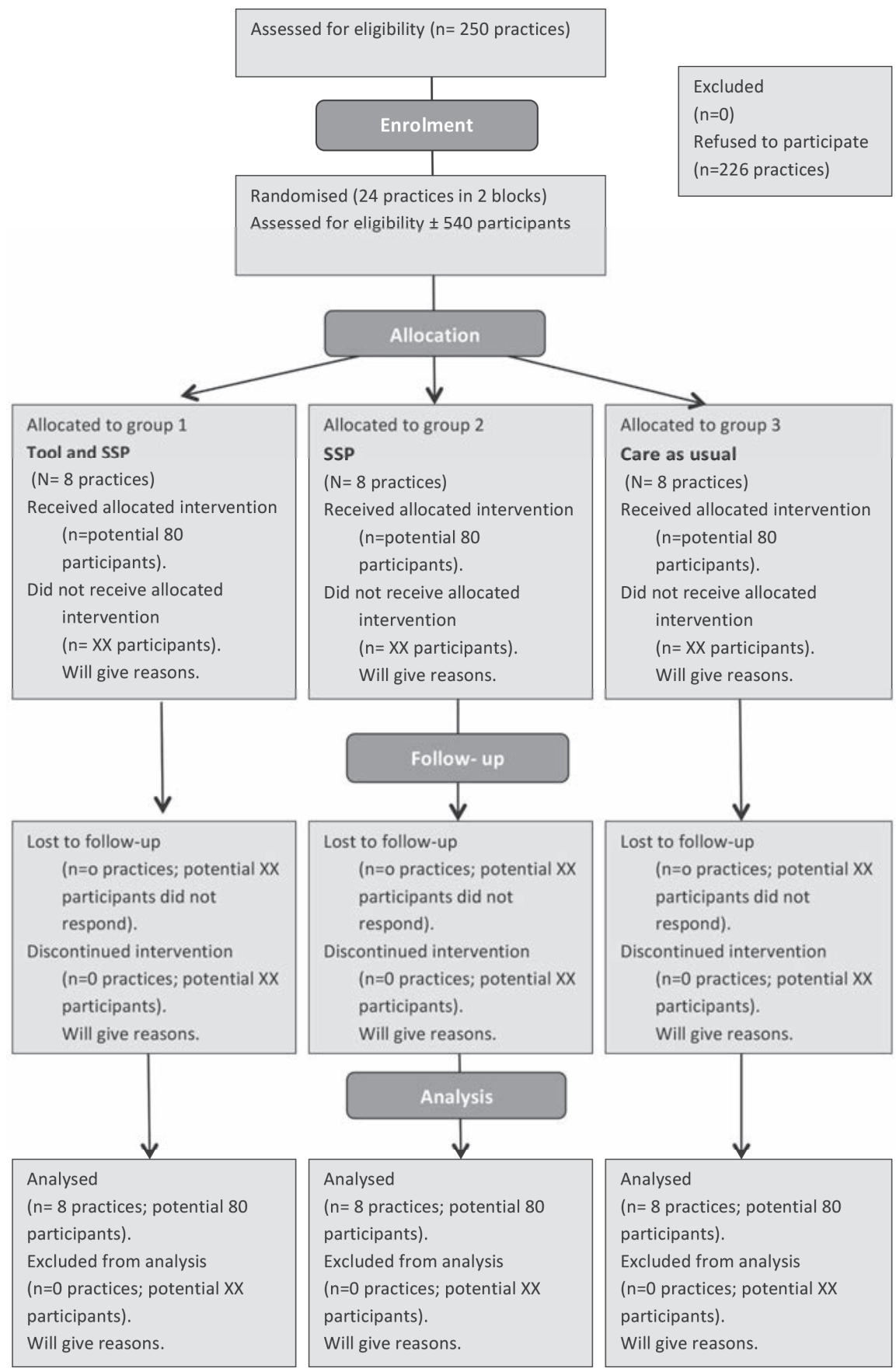

Figure 1 CONSORT flowchart trial design; potential flow of participants. 
Furthermore, patients should have access to a computer with an internet connection.

Exclusions are patients with coexisting medical conditions with a low survival rate, severe psychiatric illness or chronic disorders or diseases that seriously influence the ability to be physically active and those being primarily treated by a medical specialist or participating in another PA intervention, as well as patients with insufficient mastery of the Dutch language.

\section{Recruitment}

\section{Recruitment of practices}

GP practices located in southern regions of the Netherlands will be approached by an invitation letter, by telephone and personal contact with GP's, practice managers, and PNs, to invite them to participate in the study, until a maximum of 24 practices is reached. On the basis of the number of patients with DM2 treated per practice, the practices will be categorised into small (<90), medium (90-190), large (190-390) and extra-large (>390).

\section{Recruitment of participants}

To recruit participants for the study, PNs will identify 20-32 eligible patients per practice, who fulfil the inclusion criteria. This will be done before the randomisation of the practices. When the PN considers a patient eligible for participation, the nurse will send a recruitment letter to the patient with general information about all groups. After the randomisation, the PN will call those patients to give specific information about the group in which the practice is allocated and to ask patients if they want to participate; non-responders will be asked for their reasons not to participate. Each general practice will be instructed to include 10-14 participants, with an equal distribution of COPD and DM2 patients. When the patient decides to participate, he or she will receive an informational letter and informed consent form.

\section{Randomisation procedure}

A total of 24 practices will be randomly allocated into the three groups in two blocks of twelve practices. Before randomisation, the practices will be pre-stratified into four strata based on the size of the practice. The practices will be stratified into groups of 3 per size and randomised by an independent person into either one of the two intervention groups or the control group by numbering sealed envelopes which contain the names of the practices.

As they have to contact participating nurses to inform them about the relevant intervention, the executing researchers (S.v.d.W. \& R.V.) will be aware of which practices are in which group. Patient data will be analysed anonymously, without any recognition 
of names or practices. An independent person will store the coding key. All cleaning and processing of data will be carried out on the whole database (i.e., all three groups). The group and practice variable will only be revealed at the end of the study.

\section{Intervention}

The different components of the interventions are summarised in Figure 2.

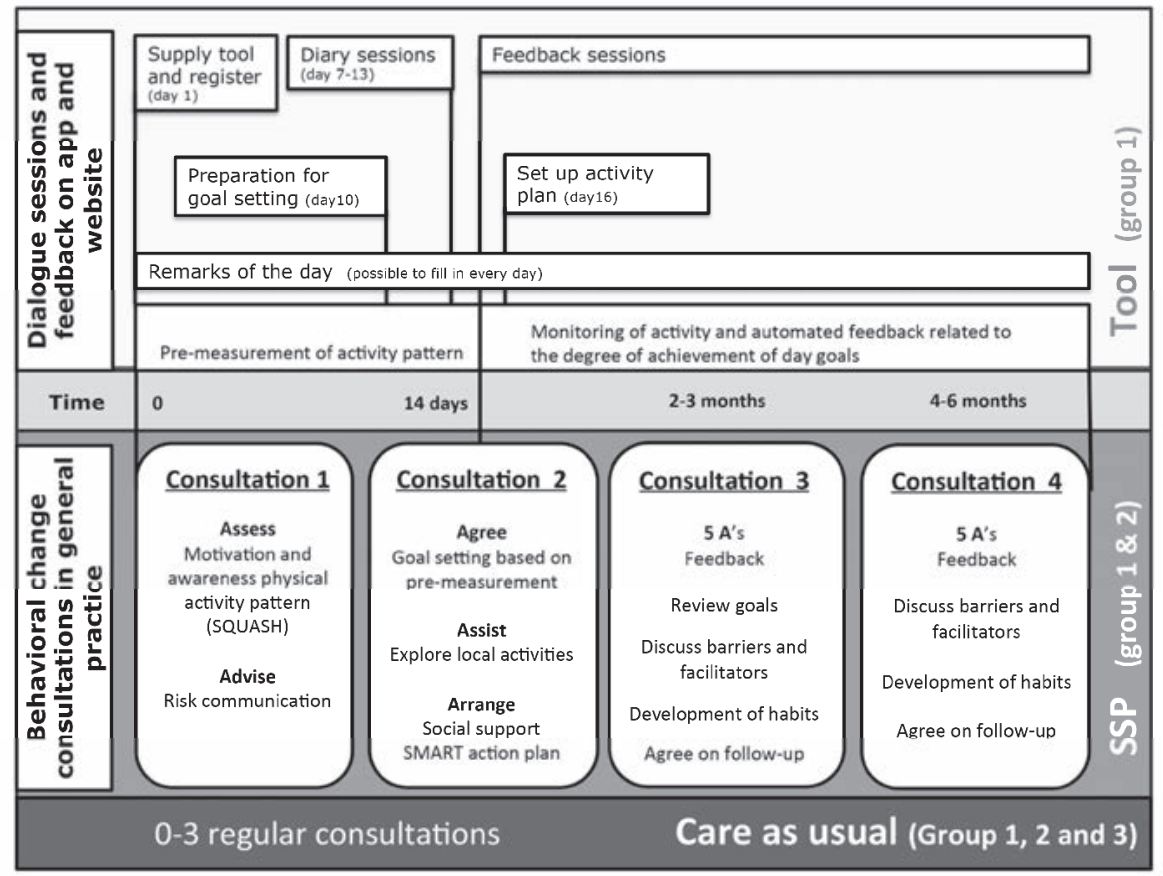

Figure 2 Interventions RCT It's LiFe!

The interventions have been designed in a user-centred manner; two patient representatives, from the Netherlands Asthma Foundation and the Dutch Diabetes Association, participated in the research group to provide feedback on every aspect of the project.

The tool (Group 1)

The It's LiFe! tool (Figure 3) consists of an accelerometer, a smartphone app, and a server/web application. Participants receive personalised feedback on the smartphone concerning their amount of activity in relation to an activity goal, which is set in dialog with their PN ${ }^{18}$ after a two week pre-measurement period. Nurses can monitor patients' PA via a secure website. ${ }^{19}$ 


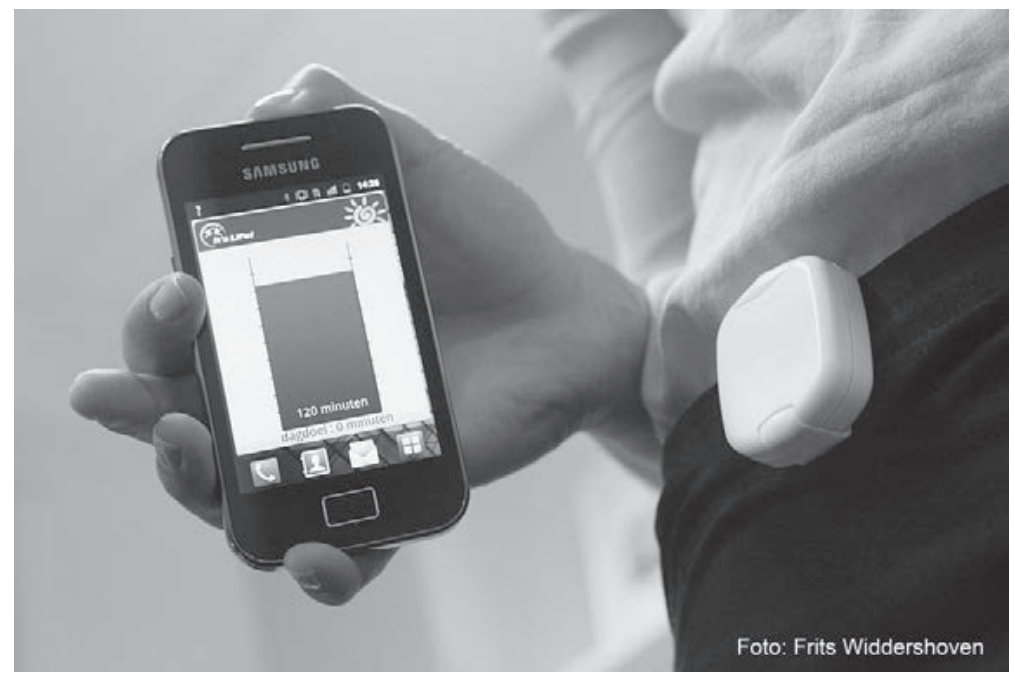

Figure 3 The It's LiFe! tool

The use of the tool starts when the participant is registered on the server by the PN. The server has two portals, one for care providers (It's LiFe! monitor) and one for patients (It's LiFe! online). The PN creates an account for the participant and then the log-in name and password are sent by email. At home, the participant has to complete a short questionnaire online (a dialog session) concerning PA preferences and has to log in on the phone. Daily at 1 a.m. the smartphone automatically connects to the server to upload the PA data from the previous day. There is a pre-measurement period of 14 days. Participants can enter 'remarks of the day' whenever they want, such as comments about being sick or having forgotten to wear the meter. In the second week, they receive dialog sessions about the enjoyment and exertion of performed activities. Furthermore, participants receive two sessions from the server concerning barriers and facilitators and activity planning based on the Physician-based Assessment and Counselling for Exercise intervention (PACE), ${ }^{22}$ with the aim of modifying factors known to influence PA, such as social support and self-efficacy. After two weeks, together the patient and nurse set a goal in minutes of activity per day, which is entered into the system by the nurse. Based on the PA data related to this goal, participants receive feedback sessions. There are several types of messages (e.g., tips, encouragement, positive trend, reward, barriers, facilitators and the suggestion to adjust goals). Participants will get such messages when they reach their target goal after 3, 5 and 14 days or when they do not reach their target after 3, 5 and 14 days. In some cases, the goals have to be reached $100 \%$ and others are based on $80 \%$ achievement. All messages are written in a positive tone, e.g., 'Good that you still try to be more active. We can see that it is hard to reach your daily target. If you want to adjust your goal, contact your care provider or click here'. 


\section{Instruction tool}

The PNs in group 1 practices will receive a personal account for the monitor, a manual and the researchers (S.v.d.W. \& R.V.) will instruct PNs on how to use the system. These researchers will also advise the nurses to try out the tool themselves and to sign up as a patient in the system to get familiar with it. In addition to a manual, there are several short instructional films available on a special website; the films cover a variety of topics, for example, how to log on to the app and how to respond to a session. For technical questions about the use of the tool, participating patients and PNs are able to contact a helpdesk during working hours.

\section{The Self-management Support Programme (Groups 1 and 2)}

The intervention in group 1 consists of the use of the tool in daily living, intertwined with consultations with the PN - the Self-management Support Programme (SSP). The intervention in group 2 consists of this programme without the use of the tool. The programme is based on the Five A's model (Assess, Advise, Agree, Assist, Arrange), a counselling protocol to support self-management in a primary care setting. ${ }^{23,24}$

This programme consists of four consultations with the PN: in the first week, after 2 weeks, after 8-12 weeks and after 16-24 weeks. Before the consultations, the participants receive an informational booklet about the course of the intervention containing the Short Questionnaire to Assess Health-enhancing PA (SQUASH) ${ }^{25}$ and a list of locally organised PA options. The duration of the consultations is 20 minutes, or a 10minute extension of a regular consultation. In the first consultation, the PN will try to increase awareness of the PA pattern of the patient, and inform the patient about the health risks related to a sedentary lifestyle. The patient and the PN will get an idea about the PA level of the patient by discussing the previously completed SQUASH questionnaire. Furthermore, the patient gets a leaflet with disease specific information related to $P A{ }^{26,27}$

During the second consultation, a goal will be set regarding physical activity in minutes per day, based on the results of the measurements of the first two weeks (premeasurement). The pre-measurement in group 1 is an objective measurement based on the tool, in group 2 this is a subjective measure achieved by asking participants to keep a PA diary. The results of the pre-measurement of group 1 are visible for the nurse on the monitor portal of the It's LiFe! server. In both intervention groups, the nurse will encourage the patient to focus on goals that fit the patient's preferences and to set up a Specific, Measureable, Attainable, Realistic, and Timely (SMART) plan to reach personal goals, and the nurse will inform the participant about locally organised exercise opportunities. 
Table 1 Details of the tool and the PA counselling consultations and proposed Behavioural Change Techniques ${ }^{28}$

\begin{tabular}{|c|c|c|}
\hline & Proposed Behavioural Change Techniques (BCT) & $\begin{array}{l}\text { Number according to } \mathrm{BCT} \\
\text { Taxonomy Abraham and Michie }\end{array}$ \\
\hline \multicolumn{3}{|c|}{ Condition 1: Tool } \\
\hline \multirow{3}{*}{$\begin{array}{l}\text { Tool widget } \\
\text { (continuous) }\end{array}$} & Prompt specific goal setting & 10 \\
\hline & Provide feedback on performance & 13 \\
\hline & Prompt review of behavioural goals & 11 \\
\hline \multirow[t]{6}{*}{ Tool sessions } & Provide general encouragement & 6 \\
\hline & Provide general information & 1 \\
\hline & Provide information on consequences & 2 \\
\hline & Prompt intention formation & 4 \\
\hline & Plan social support/social change & 20 \\
\hline & Prompt barrier identification & 5 \\
\hline \multicolumn{3}{|c|}{ Condition 1 and 2: Self-management Support Programme } \\
\hline \multirow[t]{5}{*}{ Consultation 1} & Provide general information & 1 \\
\hline & Motivational interviewing & 24 \\
\hline & Provide general encouragement & 6 \\
\hline & Provide information on consequences & 2 \\
\hline & Prompt intention formation & 4 \\
\hline \multirow[t]{4}{*}{ Consultation 2} & Provide general encouragement & 6 \\
\hline & Motivational interviewing & 24 \\
\hline & Prompt specific goal setting & 10 \\
\hline & Plan social support/social change & 20 \\
\hline \multirow[t]{6}{*}{ Consultation 3} & Provide general encouragement & 6 \\
\hline & Provide feedback on performance & 13 \\
\hline & Motivational interviewing & 24 \\
\hline & Prompt review of behavioural goals & 11 \\
\hline & Prompt barrier identification & 5 \\
\hline & Relapse prevention & 23 \\
\hline \multirow[t]{6}{*}{ Consultation 4} & Provide general encouragement & 6 \\
\hline & Provide feedback on performance & 13 \\
\hline & Motivational interviewing & 24 \\
\hline & Prompt review of behavioural goals & 11 \\
\hline & Prompt barrier identification & 5 \\
\hline & Relapse prevention & 23 \\
\hline
\end{tabular}

In the third consultation, possibly by mail or telephone, the nurse will discuss the results, barriers and facilitators related to PA. In the last consultation, the nurse will discuss the results, behaviour change(s) and habits with the participant. The proposed behaviour 
change counselling techniques have been classified according to Abraham and Michie's taxonomy as listed in Table $1 .^{28}$

Instruction for SSP

Informational booklets are produced, focusing on PA behaviour change, with an explanation and a timeline of the intervention. Before the start of the intervention, these booklets will be sent to participants.

The nurses in group 1 and 2 practices will receive a personal instruction at their workplace; these instructions will also be available as an online web lecture. The nurses will receive an information file with detailed instruction charts for the course of each consultation, and an explanation of the intended counselling techniques.

Care as usual (group 3)

Care as usual (for all three groups) consists of regular consultations with the PN (COPD patients have 1-2 consultations and DM2 patients have 4 consultations per year). Participants in the usual care group will not be offered any programme besides usual contacts with the GP and PN.

\section{Data collection}

All participants are asked (by a letter from the researchers) to wear the Pam and complete questionnaires at three different time points; namely at baseline (t0), at the end of the intervention after 4-6 months (t1), and at follow-up, 3 months after the end of the intervention (t2). Measurements and time points are summarised in Table 2.

Table 2 Measurements and time points

\begin{tabular}{|c|c|c|c|c|c|c|}
\hline \multirow{2}{*}{$\begin{array}{l}\text { Concept } \\
\text { (questionnaires) }\end{array}$} & \multicolumn{3}{|c|}{ Intervention groups } & \multicolumn{3}{|c|}{ Control group } \\
\hline & to & $t 1$ & $t 2$ & to & $t 1$ & $t 2$ \\
\hline Demographic variables & $x$ & & & $x$ & & \\
\hline Physical activity (Pam) & $x$ & $x$ & $x$ & $x$ & $x$ & $x$ \\
\hline Quality of life (SF 36) & $x$ & $x$ & $x$ & $x$ & $x$ & $x$ \\
\hline General Self-Efficacy (GSS) & $x$ & $x$ & $x$ & $x$ & $x$ & $x$ \\
\hline Exercise Self-Efficacy (ESS) & $x$ & $x$ & $x$ & $x$ & $x$ & $x$ \\
\hline Health status (DSC-R or CRQ-SAS) & $x$ & $x$ & $x$ & $x$ & $x$ & $x$ \\
\hline Process evaluation & & $x$ & & & & \\
\hline
\end{tabular}

Pam: Personal Activity Monitor

DSC-R: Diabetes Symptom Checklist-Revised

CRQ-SAS: Chronic Respiratory Questionnaire-Self-Administered Standardised

to - baseline

t1 - after 4-6 months (end of intervention)

t2 - after 9 months (post intervention) 


\section{Outcome parameters}

\section{Primary outcome measure}

\section{Physical activity}

PA will be measured with the Personal Activity Monitor (Pam AM300). ${ }^{29}$ The Pam is a small tri-axial accelerometer that can be easily attached to a belt and is worn on the hip. The Pam registers all hip movements that are made during a day. Via a docking station, and connection to the internet, the Pam scores and data of minutes a day in a sedentary category (< 1.8 METS), a living category (1.8-3 METS), a moderate category (3-6 METS), and a vigorous category (>6 METS) will be uploaded. ${ }^{29}$ The number of minutes of PA in the moderate and vigorous category (>3 METS) will be considered as the primary outcome measure. We will also report about the number of minutes of PA in the living, moderate and vigorous category $>1.8$ METS. These measures indicate all types of activity during the day. The possibility for the users of noticing their activity scores on the Pam will be deactivated; the displays will only show a digital clock. Participants will be asked to wear the Pam during 8 consecutive days for more than 12 hours a day. They will be asked to register the days and times that they wear the Pam; activities that are difficult to measure (swimming, cycling and strength training) will be recorded on a paper log. A measurement will be considered valid if the wear time is $>8$ hours per day and if there is data of $>5$ days.

\section{Secondary outcome measures}

\section{Quality of life}

To measure the quality of life the SF-36 will be used. ${ }^{30,31}$ The SF-36 consists of 36 items, organised into 8 subscales, including vitality, physical functioning, body pain, general health perceptions, emotional role functioning, social role functioning, and mental health. A higher score indicates a better quality of life.

\section{Self-efficacy}

An important mediator of PA behaviour is self-efficacy; therefore this will be measured with two different questionnaires. The 10-item General Self-efficacy Scale (GSS) is designed to assess optimistic self-beliefs to cope with a variety of difficult demands in life, scores for each item range from 1 (totally disagree) to 4 (totally agree). ${ }^{32}$ The Exercise Self-efficacy Scale (ESS) describes 18 situations during which it could be difficult to adhere to an exercise routine, for example 'without support from family and friends'. Participants are asked to rate their degree of confidence to continue with regular exercise in the listed situations. The ESS uses a 100-point scale for each item, ranging from 0 'I cannot do this at all' to 100 'I am certain that I can do it', with higher scores reflecting higher levels of exercise self-efficacy. ${ }^{33-35}$ 


\section{Additional measures}

\section{Health status}

Personal reported health status will be measured by two disease specific questionnaires, the Diabetes Symptom checklist-revised (DSC-R) for participants with DM2 and the Chronic Respiratory Questionnaire (CRQ) for participants with COPD.

DSC-R consists of 34 items and 8 sub-dimensions; hyperglycaemia, hypoglycaemia, psychological - cognitive, psychological - fatigue, cardiovascular, neurological -pain, neurological - sensoric and ophthalmological. On the DSC-R, patients indicate for each of the 34 listed symptoms whether or not they suffered from it in the last month. If they did experience the symptom, patients rate the perceived burden on a scale from 1 (not at all) to 5 (extremely). ${ }^{36-38}$

The Chronic Respiratory Questionnaire (CRQ-SAS) consists of 20 items across four dimensions: dyspnoea, fatigue, emotional function, and mastery (the patient's feeling of control over their disease). The dyspnoea portion is individualised for each patient: the person is asked to select the five activities associated with breathlessness that they perform frequently and are most important to them. Dyspnoea items can be selected from a list of 26 suggested items or may be written in by the patients. Items are scored from 1 (most severe) to 7 (no impairment). ${ }^{39,40}$

\section{Process evaluation}

Because of the expected wide range of differences in the performance of the intervention by the PNs and in the adherence of patients in using the tool, a process evaluation is necessary. ${ }^{41,42}$ The purpose of the process evaluation is to examine the context, implementation and receipt of the intervention. The evaluation consists of registration forms, a process evaluation questionnaire for participants in the intervention groups at $\mathrm{t} 1$, interviews by telephone with the PNs responsible for the study and a focus groups with PNs at the end of the study. During the interviews, information is gathered about the inclusion of participants, the course of the consultations, the education and motivation of the PNs, experienced motivation and treatment possibilities of the participants and the perceived effect of the intervention. Time spent on the intervention is recorded on registration forms. In the questionnaires, participants in both groups and the PNs are asked about their experiences with the SSP and the tool. All process evaluation components, operationalization, and measurements are summarised according to the framework of Saunders. ${ }^{43}$

\section{Sample size and power calculation}

For this study, 240 patients are required, with a minimum of 80 participants per group. Based on a validation study, we assume that the PA level of participants is an average of 24 minutes with a range of 14.6 minutes. A mean difference between group 1 and group 
3 of ten minutes (42\%) of moderate to vigorous PA spent per day will be seen as clinically relevant. While assuming an intra-class correlation of $10 \%$ based on practice, to account for the dependency of the data, with a power of $80 \%$ and a significance level of 0.05 , a total of 72 patients over 8 general practices are required in each group. Because a drop-out rate of $10 \%$ is expected, practices will be asked to include 8-14 patients per practice in each subgroup, depending on the size of the practice.

\section{Planned statistical analyses}

\section{Descriptive statistics}

Demographic data (e.g., age, gender, disease, co-morbidities) will be described for the total group and for the subgroups separately. Continuous variables will be denoted with means and standard deviations. Categorical variables will be denoted in numbers and percentages. The participants included in the 3 arms will be tested on differences between characteristics, with chi-square and ANOVA with Bonferroni-adjustment.

If variables differ between groups, with a $p$-value $\leq 0.10$, they will be considered to be potential confounders in further analysis.

\section{Data analysis for primary and secondary outcomes}

An intention to treat analysis and a per protocol analysis will be conducted. For each outcome measure (all outcomes are continuous) data will be expressed as mean +/- SD. The between group comparisons will be analysed with multilevel analysis to account for the dependency of observations within practices; the level of statistical significance will be set at 0.05 (two-tailed). Separate models (random intercept and random slope models) will be set up for each outcome measure. The independent variables in each model are two dummy variables indicating the group, with the group of patients receiving usual care as the reference category and two dummy variables for time and their interaction effects. In addition, an extra dummy variable will be included to indicate the patient group (COPD versus DM2), to study whether the effects in COPD patients differ from the effects in patients with DM2. We will also add interaction variables into the model. If needed, additional baseline variables will be included to account for possible confounding. If normality assumptions are violated, outcome variables will be log-transformed and if necessary non-parametric tests will be used. SPSS, version 19 and Mlwin, version 2.02 will be used to analyse the data.

\section{Data analysis process evaluation}

Quantitative data will be analysed by means of descriptive statistics. In order to identify relevant themes, qualitative data (results of open-ended interviews and focus groups) will be independently analysed by two researchers using NViVo version 9. A concurrent triangulation strategy will be applied to confirm, cross validate and corroborate the findings. 
Procedure for accounting for missing, unused and unexpected data

Accounting for missing values on items in questionnaires will be handled according to the scoring algorithms of the questionnaires. Missing variables in follow-up data will not be imputed since it has been shown that multilevel analysis is a very flexible method for handling missing data. ${ }^{44}$

\section{Stopping rules}

There are no formal statistical stopping rules. If a patient decides to withdraw (e.g., hospital admission), the nurse may discontinue the intervention, but all participants will be asked to complete follow-up assessments. Patients can withdraw from the study at any time.

\section{Ethical principles}

The study protocol was approved by the research ethics committee of azM/UM, Maastricht, the Netherlands in 2013 (METC12-3-071).

\section{Discussion}

This study fills a gap in the literature about how to improve self-management of patients with COPD or DM2 in increasing their level of PA by using technology embedded in primary care.

Post-recruitment selection bias, a well-known problem of cluster randomised controlled trials, will be partly avoided by asking the nurses to include patients and send a general invitation letter before the randomisation of the practices. But not informing the patients about the intended intervention (the randomisation outcome of their GP practice), is insuperable because patients have to be informed about the intervention before they agree to participate.

During a pragmatic trial, which aims to measure the effectiveness of an intervention in routine practice, it is important to collect process data to avoid Type III errors (evaluating an intervention that was inadequately implemented). In choosing the outcomes and measurements of the process evaluation, the potential for increased Hawthorne effects will be taken into account by minimising the contacts between researchers and participants, and by avoiding overlapping roles between researchers and PNs, for example by asking the PNs to include patients for the study, and by arranging an independent helpdesk. Patients will not be interviewed during the intervention in order to distinguish between the intervention and its evaluation. 


\section{Conclusion}

In conclusion, the need to increase the level of PA in people with COPD or DM2 is evident, in which the use of a monitoring and feedback tool embedded in a counselling protocol can play an important role. In the present three-arm cluster randomised controlled trial, we will evaluate the effectiveness of this counselling protocol and the surplus of using the It's LiFe! monitoring and feedback tool.

\section{Abbreviations}

COPD: Chronic Obstructive Pulmonary Disease; DM2: Type 2 Diabetes Mellitus; It's LiFe!: Interactive Tool for Self-management Through Lifestyle Feedback;

GP: General Practitioner; Pam: Physical Activity Monitor AM300; PA: Physical Activity SSP:Self-management Support Programme

\section{Competing interests}

The author(s) declare that they have no competing interests.

\section{Author's contributions}

LdW, TvdW, MS, HT, SvdW and RV conceived and designed the study. SvdW and RV are collecting the data. SvdW, RV and MS will analyse the data. RV wrote the paper. All authors edited, revised and approved the final manuscript.

\section{Acknowledgements}

The project is funded by ZonMw (The Netherlands Organisation for Health Research and development) in the programme 'New Instruments for Healthcare'.

The companies involved in the development of the tool are:

- IDEE Maastricht UMC+ Universiteitssingel 50, 6229 ER Maastricht, the Netherlands, www.idee-mumc.nl

- Maastricht Instruments Ltd. Oxfordlaan 70, 6229 EV Maastricht, the Netherlands, www.maastrichtinstruments.nl

- Sananet Care Ltd. Rijksweg Zuid 22A, 6131 AP Sittard, the Netherlands, www.sananet.nl

We would like to thank the patient representatives, Jos Donkers and Ina van Opstal, for sharing their time, thoughts and experience with us and for their critical remarks. 


\section{References}

1. World Health Organization. Global health risks : mortality and burden of disease attributable to selected major risks. Geneva, Switzerland: World Health Organization; 2009.

2. World Health Organization. Global recommendations on physical activity for health. 2010; http://www.who.int/dietphysicalactivity/factsheet_recommendations/en/. Last accessed February 20, 2015.

3. Nederlandse Diabetes Federatie. NDF care standard: transparancy and quality of diabetic care for people with diabetes type 2 [NDF Zorgstandaard: transparantie en kwaliteit van diabeteszorg voor mensen met diabetes type 2] Amersfoort: Nederlandse Diabetes Federatie; 2007.

4. Long Alliantie Nederland. Carestandard COPD [Zorgstandaard COPD]. Amersfoort: Long Alliantie Nederland; 2013.

5. Heijmans M, Spreeuwenberg P, Rijken M: Ontwikkelingen in de zorg voor chronisch zieken Rapportage 2010 [Developments in the care of chronically ill Reporting 2010] Utrecht: NIVEL; 2010. www. nivel.nl Last accessed February 20, 2015.

6. NISB. 30minutenbewegen beweegtest. [30 minutes movement activities test] 2010; http://www.30minutenbewegen.nl/ home-ik-wil-bewegen/meten-weten/beweegtest.html. Last accessed February 20, 2015.

7. Khan KM, Weiler R, Blair SN. Prescribing exercise in primary care. BMJ. 2011;343:d4141.

8. Heijmans MJWM, Spreeuwenberg P, Rijken PM. Monitor zorg-en leefsituatie van mensen met astma en mensen met COPD : trends en ontwikkelingen over de periode 2001-2008. Utrecht: NIVEL; 2009.

9. Gruijters N. NHG/LHV-Position paper: the supportive team for general practices [NHG/LHV-Standpunt Het (ondersteunend) team in de huisartsen-voorziening] http://lhv.artsennet.nl/web/file?uuid=2c3cb1e1-9b2c-4882-9def-8e68efd204b6\&owner=49c241e9-19cb4bfd-af2d-b1d0a328d6de\&contentid=127436. Last accessed February 20, 2015.

10. Orrow G, Kinmonth AL, Sanderson S, Sutton S. Effectiveness of physical activity promotion based in primary care: systematic review and meta-analysis of randomised controlled trials. BMJ. 2012;344:e1389.

11. Broekhuizen K, Kroeze W, van Poppel MN, Oenema A, Brug J. A systematic review of randomized controlled trials on the effectiveness of computer-tailored physical activity and dietary behavior promotion programs: an update. Ann. Behav. Med.Oct 2012;44(2):259-286.

12. Tudor-Locke $C$, Lutes $L$. Why do pedometers work?: a reflection upon the factors related to successfully increasing physical activity. Sports Med. 2009;39(12):981-993.

13. Neidrick TJ, Fick DM, Loeb SJ. Physical activity promotion in primary care targeting the older adult. J Am Acad Nurse Pract. Jul 2012;24(7):405-416.

14. Marcus BH, Ciccolo JT, Sciamanna CN. Using electronic/computer interventions to promote physical activity. Br J Sports Med. Feb 2009;43(2):102-105.

15. Foster C, Hillsdon M, Thorogood M, Kaur A, Wedatilake T. Interventions for promoting physical activity. Cochrane Database of Systematic Reviews 2005, Issue 1. Art. No.: CD003180.

16. McDermott MS, While AE. Maximizing the healthcare environment: a systematic review exploring the potential of computer technology to promote self-management of chronic illness in healthcare settings. Patient Educ Couns. Jul 2013;92(1):13-22.

17. van Stralen MM, de Vries H, Mudde AN, Bolman C, Lechner L. The long-term efficacy of two computertailored physical activity interventions for older adults: main effects and mediators. Health Psychol. Jul 2011;30(4):442-452.

18. van der Weegen S, Verwey R, Spreeuwenberg M, Tange H, van der Weijden T, de Witte L. The Development of a Mobile Monitoring and Feedback Tool to Stimulate Physical Activity of People With a Chronic Disease in Primary Care: A User-Centered Design. JMIR Mhealth Uhealth 2013;1(2):e8.

19. Verwey R, van der Weegen S, Tange H, Spreeuwenberg M, van der Weijden T, de Witte L. Get moving: the practice nurse is watching you! A case study of the user-centred design process and testing of a webbased coaching system to stimulate the physical activity of chronically ill patients in primary care. Inform Prim Care. 2012;20(4):289-298. 
20. Verwey R, van der Weegen S, Spreeuwenberg M, Tange H, van der Weijden T, de Witte L. A pilot study of a tool to stimulate physical activity in patients with COPD or type 2 diabetes in primary care. J Telemed Telecare. 2014 2014;20(1):29-34.

21. Campbell MK, Piaggio G, Elbourne DR, Altman DG. Consort 2010 statement: extension to cluster randomised trials. BMJ. 2012;345:e5661.

22. Calfas KJ, Sallis JF, Oldenburg B, Ffrench M. Mediators of change in physical activity following an intervention in primary care: PACE. Prev Med. May-Jun 1997;26(3):297-304.

23. Peterson JA. Get moving! Physical activity counseling in primary care. J Am Acad Nurse Pract. Jul 2007;19(7):349-357.

24. Meriwether RA, Lee JA, Lafleur AS, Wiseman P. Physical activity counseling. Am Fam Physician. Apr 15 2008;77(8):1129-1136.

25. Wendel-Vos GC, Schuit AJ, Saris WH, Kromhout D. Reproducibility and relative validity of the short questionnaire to assess health-enhancing physical activity. J Clin Epidemiol. Dec 2003;56(12):1163-1169.

26. NISB, 2010. Sportief bewegen met diabetes. [sports with diabetes] http://www.sportzorg.nl/_asset/_public/Files/Sportblessures/Diabetes_30minbewegen.pdf. Last accessed February 20, 2015.

27 NISB, 2010. Sportief bewegen met een longaandoening.[sports with a chronic lung disease] http://www.30minutenbewegen.nl/themas/gezondheid/downloads/sportief-bewegen-met-eenlongaandoening.pdf. Last accessed February 20, 2015.

28. Abraham C, Michie S. A Taxonomy of Behavior Change Techniques Used in Interventions. Health Psychol. 2008;27(3):379-387.

29. Slootmaker SM, Chin APMJ, Schuit AJ, van Mechelen W, Koppes LL. Concurrent validity of the PAM accelerometer relative to the MTI Actigraph using oxygen consumption as a reference. Scand J Med Sci Sports. Feb 2009;19(1):36-43.

30. Aaronson NK, Muller M, Cohen PD, et al. Translation, validation, and norming of the Dutch language version of the SF-36 Health Survey in community and chronic disease populations. J Clin Epidemiol. Nov 1998;51(11):1055-1068.

31. Van der Zee KI, Sanderman R, Heyink J. A comparison of two multidimensional measures of health status: the Nottingham Health Profile and the RAND 36-Item Health Survey 1.0. Qual Life Res. Feb 1996;5(1):165174.

32. Schwarzer R, Jerusalem M. pp35-37 in Weinman J, Wright S, Johnston M, eds. Measures in Health Psychology: a user's portfolio. Windsor, NFER-NELSON, 1995.

33. van der Heijden MM, Pouwer F, Pop VJ. Psychometric Properties of the Exercise Self-efficacy Scale in Dutch Primary Care Patients with Type 2 Diabetes Mellitus. Int J Behav Med. Apr 32013.

34. Shin Y, Jang H, Pender NJ. Psychometric evaluation of the exercise self-efficacy scale among Korean adults with chronic diseases. Res Nurs Health. Feb 2001;24(1):68-76.

35. Everett B, Salamonson Y, Davidson PM. Bandura's exercise self-efficacy scale: validation in an Australian cardiac rehabilitation setting. Int J Nurs Stud. Jun 2009;46(6):824-829.

36. Arbuckle RA, Humphrey L, Vardeva K, et al. Psychometric Evaluation of the Diabetes Symptom ChecklistRevised (DSC-R)A Measure of Symptom Distress. Value Health. 2009;12(8):1168-1175.

37. Grootenhuis PA, Snoek FJ, Heine RJ, Bouter LM. Development of a type 2 diabetes symptom checklist: a measure of symptom severity. Diabet Med. Apr 1994;11(3):253-261.

38. Secnik Boye K, Matza LS, Oglesby A, et al. Patient-reported outcomes in a trial of exenatide and insulin glargine for the treatment of type 2 diabetes. Health Qual Life Outcomes. 2006;4:80.

39. Rutten-van Molken M, Roos B, Van Noord JA. An empirical comparison of the St George's Respiratory Questionnaire (SGRQ) and the Chronic Respiratory Disease Questionnaire (CRQ) in a clinical trial setting. Thorax. Nov 1999;54(11):995-1003.

40. Glaab T, Vogelmeier C, Buhl R. Outcome measures in chronic obstructive pulmonary disease (COPD): strengths and limitations. Respir Res. 2010;11.

41. Hasson H. Systematic evaluation of implementation fidelity of complex interventions in health and social care. Implement Sci. 2010;5(1). 
42. Linnan L, Steckler A: Process evaluation for Public Health Interventions Research. An overview. In Process evaluation for Public health interventions research. Edited by Linnan L, Steckler A. San Francisco: JosseyBass; 2002:1-23.

43. Saunders RP. Developing a Process-Evaluation Plan for Assessing Health Promotion Program Implementation: A How-To Guide. Health Promot Pract. 2005;6(2):134-147.

44. Twisk JWR. Applied multilevel analysis : a practical guide. Cambridge, UK; New York: Cambridge University Press; 2006. 

It's LiFe! mobile and web-based monitoring and feedback tool embedded in primary care increases physical activity: a cluster randomised controlled trial

This chapter was published as:

van der Weegen S \& Verwey R, Spreeuwenberg M, Tange H, van der Weijden T, de Witte L. It's LiFe! Mobile and Web-Based Monitoring and Feedback Tool Embedded in Primary Care Increases Physical Activity: A Cluster Randomised Controlled Trial. J Med Internet Res. 2015;17(7):e184 


\section{Abstract}

Background Physical inactivity is a major public health problem. The It's LiFe! monitoring and feedback tool embedded in the Self-Management Support Programme (SSP) is an attempt to stimulate physical activity in people with chronic obstructive pulmonary disease or type 2 diabetes treated in primary care.

Objective Our aim was to evaluate whether the SSP combined with the use of the monitoring and feedback tool leads to more physical activity compared to usual care and to evaluate the additional effect of using this tool on top of the SSP.

Design A three-armed cluster randomised controlled trial. Twenty four family practices were randomly assigned to one of three groups in which participants received the tool + SSP (group 1), the SSP (group 2), or care as usual (group 3).

Methods The secondary outcomes were general and exercise self-efficacy and quality of life. Outcomes were measured at baseline, after the intervention (4-6 months), and 3 months thereafter.

Results The group that received the entire intervention (tool + SSP) showed more physical activity directly after the intervention than group 3 (mean difference 11.73, $95 \% \mathrm{Cl} 6.21$ to $17.25 ; \mathrm{P}<0.001$ ), and group 2 (mean difference $7.86,95 \% \mathrm{Cl} 2.18$ to 13.54 ; $\mathrm{P}=0.003)$. Three months after the intervention this effect was still present and significant (compared to group 3: mean difference 10.59, 95\% Cl 4.94 to 16.25; $\mathrm{P}<0.001$; compared to group 2: mean difference $9.41,95 \% \mathrm{Cl} 3.70$ to $15.11 ; \mathrm{P}<0.001)$. There was no significant difference in effect between group 2 and group 3 on both time points. There was no interaction effect for disease type.

Conclusion The combination of counselling with the tool proved an effective way to stimulate physical activity. Counselling without the tool was not effective. Future research about the cost-effectiveness, application under more tailored conditions and in other target groups is recommended.

Trial registration ClinicalTrials.gov: NCT01867970 


\section{Introduction}

Physical inactivity is a major public health problem ${ }^{1,2}$ because it increases the risk of several diseases, such as coronary heart disease, type 2 diabetes, and several types of cancer. It also shortens life expectancy. ${ }^{1}$ For people with a chronic disease, physical inactivity enhances the chance of complications and comorbidities. ${ }^{3}$ Unfortunately, about one-third of adults worldwide do not reach public health guidelines for recommended levels of physical activity (PA). ${ }^{4}$ Therefore, the promotion of PA is a public health priority. ${ }^{5}$ One of the approaches to increase PA is through primary health care. ${ }^{6}$ Because practice nurses have frequent contact with people with chronic conditions to monitor treatment outcomes, it is recommended that they incorporate support to change physical inactivity behaviours. ${ }^{7,8}$ However, providing only verbal advice has proven to be insufficient. ${ }^{9}$ Despite the heterogeneity in results of physical activity intervention studies, the most effective approach is professional advice and guidance with continued support and combining a mix of behaviour change strategies. ${ }^{10-12}$ Effective behaviour change strategies for the promotion of PA are self-monitoring, providing feedback for behaviour, goal setting, providing tools to facilitate behaviour, action planning, social support, barrier identification, and providing information on the consequences specific to the individual. ${ }^{10,11,13}$ An example of a tool to facilitate behaviour is the use of innovative technology such as smartphones with built-in, or in combination with, pedometers or accelerometers. These technologies can facilitate selfmonitoring, goal setting, and real-time feedback. Despite, the fact that general smartphone use is growing as well as smartphone use in PA research, ${ }^{14}$ there is a lack of well-designed experimental studies with appropriate intervention periods and sample sizes $^{15}$ to explore whether these technologies add value on top of behaviour change counselling by the practice nurse (PN). The It's LiFe! intervention is a combination of behaviour change strategies delivered by the PN in a Self-management Support Programmeme (SSP) that is partly integrated with usual care as well as the use of a monitoring and feedback tool for patients in daily life.

A cluster randomised controlled trial was conducted to evaluate the longitudinal effects of this multifaceted intervention on 40-70 year old patients with chronic obstructive pulmonary disease (COPD) and diabetes type 2 (DM2) in primary care. Furthermore, the additional effect of using this tool on top of the SSP was evaluated. The main hypothesis was that after a four to six month intervention period, the complete intervention increases participants' moderate to vigorous physical activity by at least 10 minutes per day compared to care as usual, and that this increase maintains over three months. 


\section{Methods}

The study methods, intervention, and outcomes have been reported in detail previously. $^{16}$

\section{Study design}

A three-arm clustered randomised controlled trial among 24 general practices in the south of the Netherlands was conducted. A cluster design was chosen to avoid contamination by unintended influence of the $\mathrm{PN}$ in the control group. After stratification based on the number of registered DM2 patients per practice, two blocks of 12 practices were randomly assigned in three groups using sealed envelopes. Practices allocated to group 1 received the complete intervention (monitoring and feedback tool and SSP), practices in group 2 received the SSP only, whereas practices in group 3 received care as usual. Four strata were defined: small (<90 DM2-patients), medium (90-190), large (190-390), and extra-large (>390). There was no blinding for allocation of practices. The research team was blinded for allocation of participants during the analysis phase. Data were analysed anonymously and coding was revealed after analyses.

\section{Participants; practices and patients}

Two hundred and fifty family practices in the South of Netherlands were invited by invitation letter, telephone, or personal contact, until 24 practices agreed to participate. Eligibility for participants was determined as follows: between 40 and 70 years old with DM2 or COPD, and who did not, according to the PN, comply with the Dutch Norm for Healthy Exercise (having at least 30 minutes of moderate to vigorous physical activity on 5 or more days of the week). ${ }^{17}$ Additional inclusion criteria for the DM2 patients was a $\mathrm{BMI}>25$, and for the COPD patients, a clinical diagnosis of COPD according to the GOLDcriteria stage 1-3, known to be stable in their respiratory function for at least six weeks and on a stable drug regimen. Furthermore, participants needed to be able to access a computer with an internet connection and master the Dutch language sufficiently.

Exclusion criteria were the presence of coexisting medical conditions with a low survival rate, severe psychiatric illness, or chronic disorders or diseases that seriously influence the ability to be physically active, and being treated primarily by a medical specialist or participating in another PA intervention.

The PNs in each practice were asked to send 20-32 general invitation letters to patients who met the inclusion criteria. After randomisation, the PN called the patients to give specific information about the allocated condition and ask if they wanted to participate. If the patient decided to participate, they received a specific information letter and an informed consent form. Each practice was instructed to include five to 
seven patients with DM2 and five to seven patients with COPD. This study was approved by the Medical Ethical Committee of the Maastricht University/Academic Hospital Maastricht in the Netherlands (12-3-071).

\section{Intervention}

The complete It's LiFe! intervention consisted of the Self-management Support Programmeme and a monitoring and feedback tool. Both elements were developed in a user-centred design process and tested on usability and feasibility. ${ }^{18-22}$ Furthermore, two patient representatives from the Netherlands Asthma Foundation and the Dutch Diabetes Association participated in the research group to provide feedback on every aspect of the trial.

The Self-management Support Programmeme (SSP)

The programme consisted of four individual consultations with the PN; in the first week, after two weeks, after two to three months, and after four to six months (Figure 1). ${ }^{18}$ First, the participants received an information booklet about the course of the intervention containing the Short Questionnaire to Assess Health-Enhancing PA $(\mathrm{SQUASH})^{23}$ and a list of locally organised PA activities.

In the first consultation, the PN raised awareness about the risks of physical inactivity, and the PA level of the patient was discussed using the previously completed SQUASH questionnaire. In addition, participants received a general and a disease specific pamphlet about PA. ${ }^{24-26}$ Between the first and the second consultation, a premeasurement of the activity pattern was taken, and participants answered questions about barriers and facilitators for PA. In group 1, PA was objectively measured by the tool, and all questions were answered via a dialogue session on the tool. Group 2 kept a PA diary on paper and answered questions about barriers and facilitators in the information booklet. During the second consultation, a personal goal was set in minutes of activity per day based on the pre-measurement, and the PN encouraged the participants to set up an activity plan to reach personal goals. Furthermore, the nurse informed the participants about locally organised PA options. In the third consultation, possibly by mail or telephone, activity results, barriers, facilitators, and the creation of new PA habits were discussed, and some participants reconsidered their activity goal. In the last consultation, activity results, barriers, facilitators, and PA habits were evaluated. Furthermore, how the PN and patient would continue the lifestyle coaching was discussed. The consultations were based on the "Five ' $A$ 's Cycle" counselling technique (assess-advise-agree-assist-arrange). ${ }^{27,28}$ 


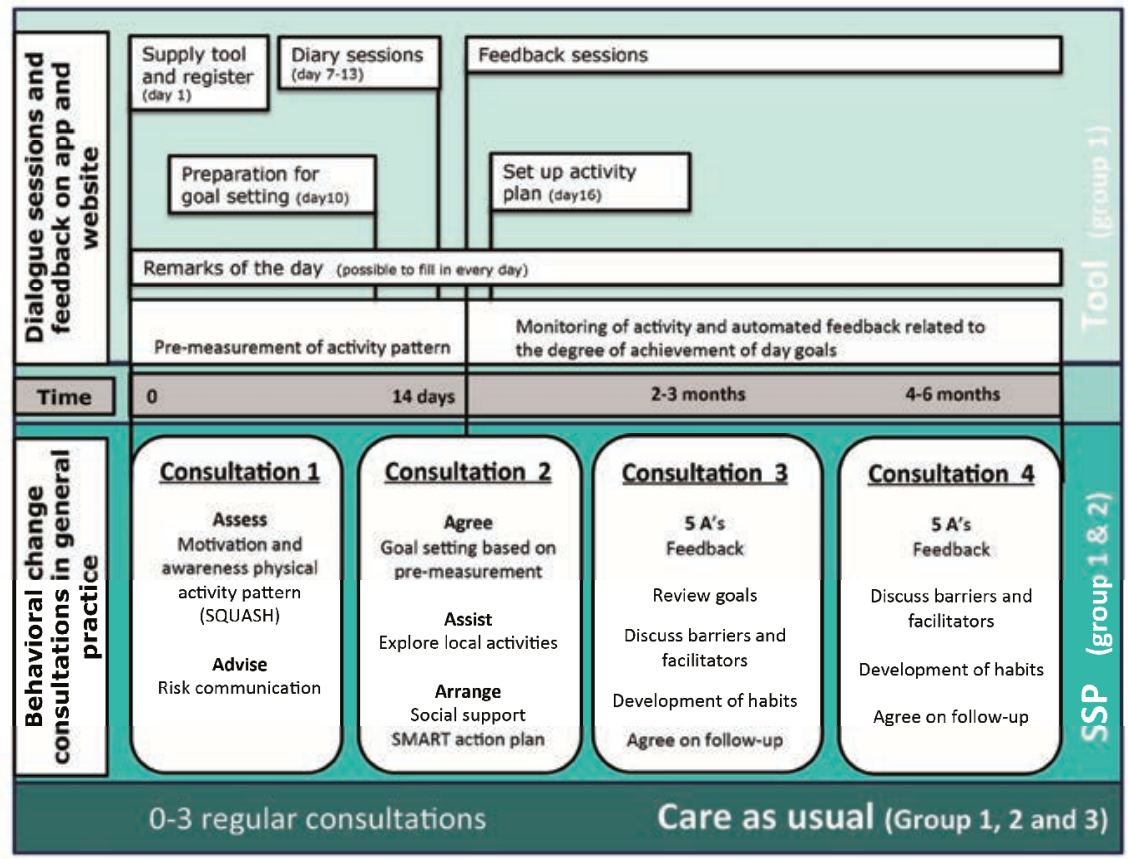

Figure 1 Course of the It's LiFe! interventions.

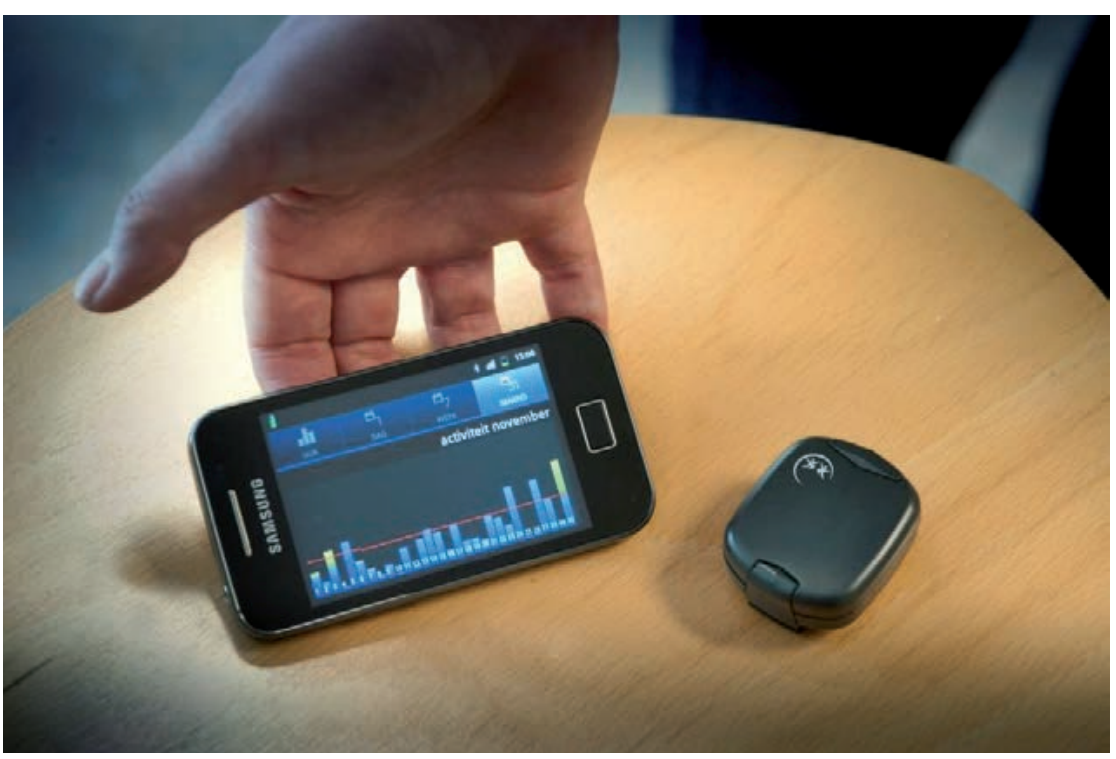

Figure 2 The It's LiFe! activity monitor and smartphone app. 
The tool

The tool consists of a 3D activity monitor, a smartphone app, and a web application (Figure 2). ${ }^{19}$ Participants were asked to wear the activity monitor on a daily basis and they could see their real time activity results and history in minutes of moderate to vigorous activity on the smartphone and web application, in relation to a personal goal. During the pre-measurement, participants participated in dialogue sessions (Figure 1). In the "diary sessions," they were asked about enjoyment and exertion of performed activities. In the "preparation for goal setting" they were asked about barriers and facilitators to exercise. Based on the activity results and the answers in the dialogue sessions, a personal activity goal was set in the second consultation of the SSP. Hereafter, automated feedback messages were sent related to the personal goal. Moreover, the participant was asked in a dialogue session to set up an activity plan to achieve the daily goal. During the entire intervention, activity results and answers to dialogue sessions were visible for the PN on a secured web application. ${ }^{19,22}$ The applications were not changed or updated during the trial (version 2.7). For technical questions and problems with the tool, the participants and PNs could contact a helpdesk during working hours to avoid contact between researchers and participants.

Training of the practice nurses

For mastering the execution of the intervention, PNs in group 1 and 2 received an online web lecture and consecutively a personal instruction session at their workplace. In addition they received on paper, an explanation of the Five A's model, the associated counselling techniques and detailed instruction charts for each consultation. Nurses in group 1 were able to try out the tool before the start of the consultations.

\section{Data collection}

All participants received a Personal Activity Monitor AM300 (Pam) ${ }^{29-31}$ and questionnaires by regular mail, at baseline (t0), after the intervention at four to six months after baseline (t1), and three months after the end of the intervention, approximately nine months after baseline (t2). The last measurement was initially set at 6 months after the intervention, but due to time and money constraints, this could not be realised. The Pam was blinded, which means that participants could not read the display with activity information to prevent any feedback and intervention effect of this measurement.

\section{Outcome measures}

The primary outcome measure was the average minutes per day of PA per patient, measured with the Pam. ${ }^{29-31}$ The participants were asked to wear the Pam for eight consecutive days clipped to their waistband on the hip, and to record in a diary the time 
it was worn. A measurement was considered valid if the tool was worn on $\geq 5$ days for $\geq 8$ hours. Minutes per day were divided in three categories according to metabolic equivalent tasks (METS): light (1.8-2.99 METS), moderate (3-6 METS), and vigorous (>6 METS). The number of minutes of PA in the moderate and vigorous category ( $\geq 3$ METS) was considered the primary outcome measure because moderate to vigorous activity is recommended by the World Health Organization. ${ }^{32}$ Secondary outcome measures were general self-efficacy (general self-efficacy scale), ${ }^{33}$ exercise self-efficacy (exercise selfefficacy scale), ${ }^{34-36}$ and quality of life (RAND 36). ${ }^{37,38}$

\section{Statistical analysis}

The sample size calculation was based on the primary outcome measure (minutes of moderate to vigorous PA per day). Based on a power of $80 \%$, an alpha of 0.05 (twotailed testing), an expected difference between group 1 and 3 of ten minutes of PA per day per participant, and an assumed intra-class correlation between the practices of 0.15, 72 participants over eight general practices were required in each group. A dropout rate of $10 \%$ was taken into account, which resulted in a desired number of 80 participants per group.

Intention to treat and per protocol analyses were performed. Participants of the intervention groups were included in the per protocol analysis if they received a minimum of three consultations (75\%) spread over at least three months based on registration forms of the consultations obtained from the PNs. Participants from all groups were excluded from the per protocol analysis if they did not complete the second measurement (t1). Per protocol analysis were conducted to investigate whether results were different if only participants were included who adhered sufficiently to the interventions.

Normal distribution of the data was checked visually using normal q-q plots and histograms. Outliers were not removed. Continuous variables were presented as means, and standard deviation and categorical variables as numbers and percentages. Differences in baseline characteristics between groups at baseline were investigated with chi-square and analysis of variance (ANOVA). Variables that differed with a P-value of 0.10 or smaller were considered as potential confounders in further analysis. For the RAND 36 outcomes only the physical component and the mental component were used in further analysis, since the eight subscales strongly correlated. To adjust for the dependency of patients within time and practices (intra class correlation [ICC]) restricted maximum likelihood (REML) multilevel analyses with random intercepts were used. The differences of the -2 log likelihood and degrees of freedom between models were examined to decide if a one, two, or three-hierarchical (time, participants, and general practices) model had to be applied (model selection was performed with a maximum likelihood [ML]). Separate models were set up for each outcome measure, adjusted with Bonferroni correction. The independent variables in each model were two dummy variables indicating the group, with the group of participants receiving care as usual as 
the reference category, and two dummy variables for time and their interaction effects. In addition, outcome estimates of the multilevel analyses were corrected for baseline and for potential confounders (differences between groups at baseline). Potential confounders were stepwise included in the model if the regression coefficients of time, group, and the interaction of group $x$ time, changed by $\geq 10 \%$ on average. To study whether the effects in COPD patients differed from the effects in participants with DM2, a subgroup analysis was done by including interaction effects. Missing values on items in questionnaires were handled according to the questionnaire's analysis manual; missing data in follow up were not imputed as multilevel analysis accounts for that. ${ }^{39}$ All analyses were carried out with IBM Statistical Product and Service Solutions (SPSS) Statistics for Windows, version 22.0.

\section{Results}

In total, twenty-four general practices were randomly assigned to group 1 (tool and SSP), group 2 (SSP), or group 3 (care as usual). In every group, one small practice, three medium, three large, and one extra-large practice were included. The individual practices included 3 to 14 participants with a median (interquartile range) of nine participants (7-10 participants). As shown in Figure 3, PNs sent approximately 540 patients a general invitation letter and 199 patients (Group 1: 65 participants, group 2: 66 participants, group 3: 68 participants) agreed to participate and completed the baseline measurement. In June 2013, the first practices started with the intervention, and in April 2014 PNs in the last practices performed their last consultations. In group 1, one participant did not start with the intervention because in his opinion, the intervention was not tailored to his age group, and 12 participants did not receive the minimal intervention as intended. In group 2, two participants dropped out before the start of the intervention and seven participants did not receive the minimal intervention as intended. In total, 23 participants were lost to follow-up. In the "intention to treat" analyses, data from all participants were taken into account $(n=199)$ (Figure 3). Table 1 shows the baseline characteristics of participants in each group, and Table 2 shows the mean outcome values at baseline. Significant group differences, which were included as confounders in further analyses, were found for Body Mass Index (BMI), computer use, minutes of PA ( $\geq 3 \mathrm{METS}$ ), and quality of life (physical component scale). 


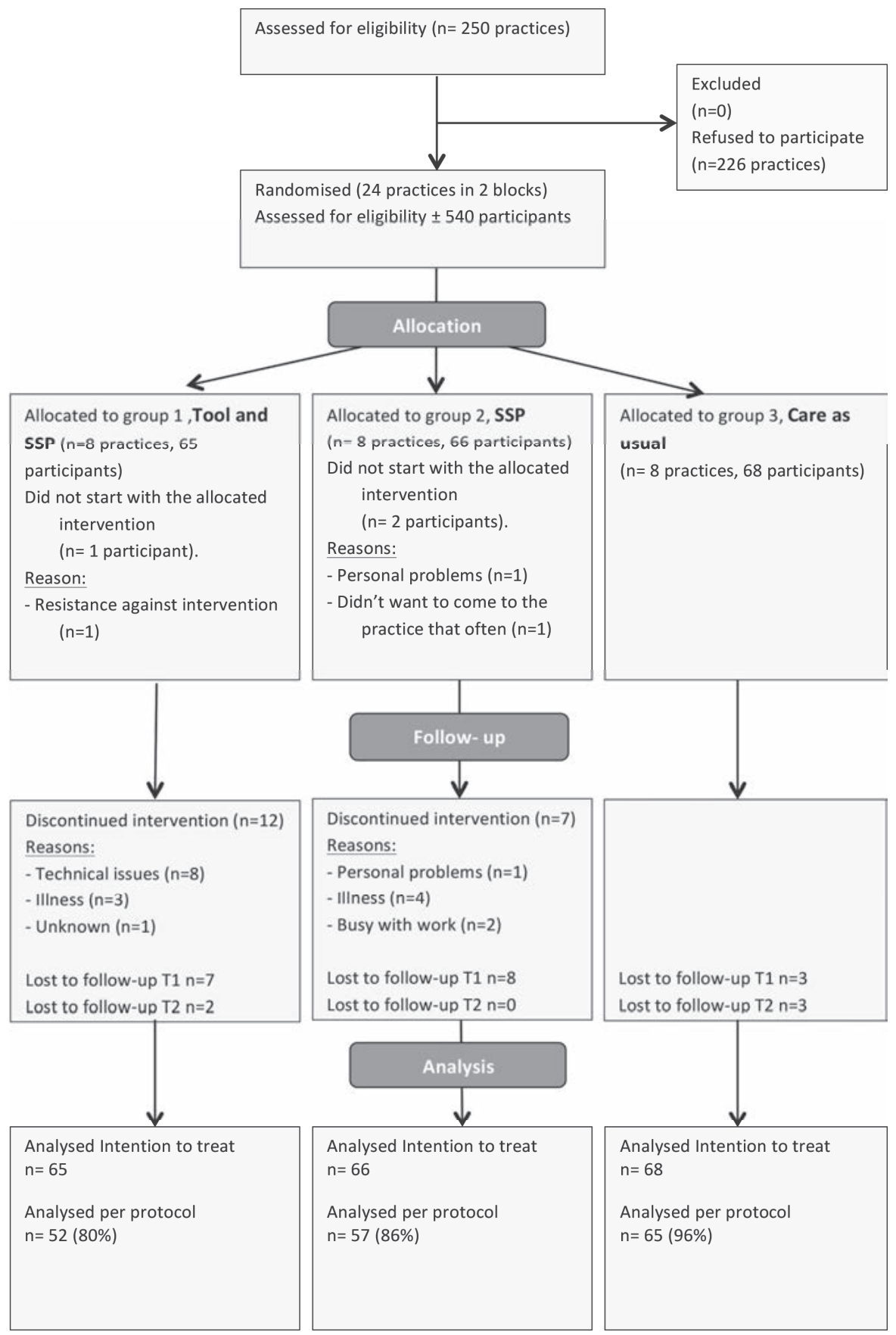

Figure 3 It's LiFe! CONSORT flow diagram. 
Table 1 Baseline characteristics of participants. Values are numbers (percentages) unless stated otherwise.

\begin{tabular}{|c|c|c|c|}
\hline \multirow[t]{2}{*}{ Characteristics of participants } & Group 1 (n=65) & Group 2 (n=66) & Group $3(n=68)$ \\
\hline & Tool \& SSP & SSP & Care as usual \\
\hline Female sex & $34(52.3)$ & $31(47.0)$ & $37(54.4)$ \\
\hline Age in years, mean (SD) & $57.5(7.0)$ & $56.9(8.3)$ & $59.2(7.5)$ \\
\hline $\mathrm{BMI}^{*}$, mean (SD) & $30.4(5.7)$ & $29.5(5.9)$ & $28.2(4.3)$ \\
\hline Origin non-Dutch & $5(7.7)$ & $4(6.1)$ & $3(4.4)$ \\
\hline Married or cohabiting partners & $48(73.9)$ & $46(69.7)$ & 55 (80.9) \\
\hline \multicolumn{4}{|l|}{ Education } \\
\hline Low & $19(29.2)$ & $19(28.8)$ & $15(22.1)$ \\
\hline Medium & $35(53.8)$ & $40(60.6)$ & $43(63.2)$ \\
\hline High & $11(16.9)$ & $6(9.1)$ & $10(14.7)$ \\
\hline Employed & $31(47.7)$ & $31(47.0)$ & $31(45.6)$ \\
\hline COPD & $25(38.5)$ & $26(39.4)$ & $31(45.6)$ \\
\hline \multicolumn{4}{|l|}{ Gold stadium } \\
\hline GOLD stadium 1 & $9(36.0)$ & $13(50.0)$ & $15(48.4)$ \\
\hline GOLD stadium 2 & $15(60.0)$ & $12(46.2)$ & $16(51.6)$ \\
\hline GOLD stadium 3 & $1(4.0)$ & $1(3.8)$ & $0(0.0)$ \\
\hline Diabetes type 2 & $40(61.5)$ & $40(60.6)$ & $37(54.4)$ \\
\hline Insulin use & $3(7.5)$ & $6(15.0)$ & $8(21.6)$ \\
\hline Co-morbidities & $51(78.5)$ & $46(69.7)$ & $43(63.2)$ \\
\hline Asthma & $6(9.2)$ & $8(12.1)$ & $4(5.9)$ \\
\hline Cardiac/vascular & $12(18.5)$ & $8(12.1)$ & $7(10.3)$ \\
\hline Hypertension & $22(33.8)$ & $29(43.9)$ & $20(29.4)$ \\
\hline Arthritis & $13(20.0)$ & $11(16.7)$ & $16(23.5)$ \\
\hline Depression & $3(4.6)$ & $5(7.6)$ & $5(7.4)$ \\
\hline Also diabetes & $2(3.1)$ & $1(1.5)$ & $1(1.5)$ \\
\hline Also COPD & $2(3.1)$ & $6(9.1)$ & $2(2.9)$ \\
\hline Other & $28(43.1)$ & $22(33.3)$ & $27(39.7)$ \\
\hline \multicolumn{4}{|l|}{ Computer use* } \\
\hline Regularly & 50 (76.9) & $43(65.2)$ & $47(69.1)$ \\
\hline Rarely & $15(23.1)$ & $23(34.8)$ & 21 (30.9) \\
\hline \multicolumn{4}{|l|}{ Mobile phone use } \\
\hline Owns a smartphone & $24(36.9)$ & $24(36.3)$ & $19(28.0)$ \\
\hline Uses mobile phone frequently & $20(30.8)$ & $20(30.3)$ & $15(22.1)$ \\
\hline Uses mobile phone rarely & $19(29.2)$ & $19(28.8)$ & $33(48.5)$ \\
\hline Does not own a mobile phone & $2(3.1)$ & $3(4.5)$ & $1(1.5)$ \\
\hline
\end{tabular}

* $P \leq 0.10$, tested with chi square or ANOVA

** $P \leq 0.05$, tested with chi square or ANOVA 
Table 2 Values at baseline. Values are means with (standard deviations).

\begin{tabular}{|c|c|c|c|}
\hline & Group 1 ( $n=65)$ & Group 2 (n=66) & Group $3(n=68)$ \\
\hline & Tool \& SSP & SSP & Care as usual \\
\hline \multicolumn{4}{|l|}{ Physical activity } \\
\hline Minutes per day in moderate and vigorous $\geq 3$ METS* & $39.3(18.1)$ & $47.5(26.5)$ & $44.1(20.3)$ \\
\hline Wear time of the Pam in hours a day & $14.3(1.7)$ & $14.5(1.5)$ & $14.3(1.3)$ \\
\hline \multicolumn{4}{|l|}{ Self-efficacy } \\
\hline General self-efficacy scale & $3.2(0.5)$ & $3.2(0.5)$ & $3.1(0.5)$ \\
\hline Exercise self-efficacy scale & $55.4(17.0)$ & $53.1(21.3)$ & $54.0(19.2)$ \\
\hline Factor 1 Situational/interpersonal & $51.2(18.7)$ & $45.9(20.8)$ & $48.3(23.2)$ \\
\hline Factor 2 Competing demands & $62.0(18.5)$ & $60.0(21.6)$ & $62.6(20.2)$ \\
\hline Factor 3 Internal feelings & $53.8(18.8)$ & $53.3(22.2)$ & $52.4(21.1)$ \\
\hline \multicolumn{4}{|l|}{ Quality of life } \\
\hline Physical Component Score* & $42.5(11.1)$ & $46.1(9.8)$ & $45.8(9.4)$ \\
\hline Mental Component Score & $48.2(10.3)$ & $48.6(11.7)$ & $50.1(9.5)$ \\
\hline RAND36 physical functioning & $68.7(22.2)$ & $74.6(20.4)$ & $74.7(21.9)$ \\
\hline RAND36 role functioning physical** & $55.8(45.9)$ & $72.2(36.7)$ & $70.8(39.5)$ \\
\hline RAND36 role functioning emotional & $72.8(38.1)$ & $77.4(34.4)$ & $78.4(35.4)$ \\
\hline RAND36 social functioning & $77.1(22.8)$ & $77.7(23.8)$ & $80.5(20.8)$ \\
\hline RAND36 body pain & $66.0(24.8)$ & $70.7(25.1)$ & $70.8(23.1)$ \\
\hline RAND36 mental health & $73.9(15.1)$ & $74.9(19.7)$ & 76.5 (14.9) \\
\hline RAND36 vitality** & $55.2(19.1)$ & $62.5(20.8)$ & $64.3(16.4)$ \\
\hline RAND36 general health & $51.3(19.6)$ & $55.6(20.6)$ & $55.2(16.2)$ \\
\hline
\end{tabular}

* $P \leq 0.10$, tested with ANOVA

** $P \leq 0.05$, tested with ANOVA

\section{Primary outcome (Intention to treat)}

For the primary outcome, a two level hierarchical model dealing with dependency of measurements in time within patients (but not family practices) was applied with a correction for baseline physical activity and wear time. ICC for repeated measures was 0.77, ICC for participants in the same practice was 0.005. Directly after the intervention, participants in group 1, who received the tool and the SSP, showed 8 minutes more moderate and vigorous physical activity ( $\geq 3$ METS) than participants in the SSP, and 12 minutes more PA than the care as usual group. This improvement difference was 9 minutes and 11 minutes, respectively, three months after the end of the intervention. No difference was observed between group 2 (SSP) and group 3 (care as usual). Results are shown in Table 3. 
Table 3 Multilevel analyses for differences between the three groups for physical activity.

\begin{tabular}{|c|c|c|c|c|c|c|c|c|}
\hline & & \multicolumn{3}{|c|}{ Unadjusted mean (SD) } & \multicolumn{3}{|c|}{ Adjusted mean difference $95 \% \mathrm{Cl}, p$-value ${ }^{\mathrm{A}}$} & \multirow[t]{2}{*}{$I C C^{B}$} \\
\hline \multirow{4}{*}{$\begin{array}{l}\mathrm{PA} \\
\text { moderate an } \\
\text { vigorous } \\
(\geq 3 \mathrm{METS})^{\mathrm{A}}\end{array}$} & $\begin{array}{l}\text { Time } \\
\text { points }\end{array}$ & ool \& SSP & SSP & CAU & $\begin{array}{c}\text { Tool \& SSP - } \\
\text { CAU }\end{array}$ & $\begin{array}{l}\text { SSP - } \\
\text { CAU }\end{array}$ & $\begin{array}{c}\text { Tool \& SSP - } \\
\text { SSP }\end{array}$ & \\
\hline & $\begin{array}{l}\text { Baseline } \\
\mathrm{d}(\mathrm{t} 0)\end{array}$ & $\begin{array}{l}39.29 \\
(18.1)\end{array}$ & $\begin{array}{l}47.47 \\
(26.5)\end{array}$ & $\begin{array}{l}44.13 \\
(20.3)\end{array}$ & $\begin{array}{l}-0.34(-5.65 \text { to } \\
4.97) ; 1.000\end{array}$ & $\begin{array}{l}0.15(-5.13 \text { to } \\
5.44) ; 1.000\end{array}$ & $\begin{array}{l}-0.50(-5.83 \text { to } \\
4.84) ; 1.000\end{array}$ & 0.77 \\
\hline & $\begin{array}{l}\text { 4-6 months } \\
\text { (t1) }\end{array}$ & $\begin{array}{l}48.16 \\
(23.8)\end{array}$ & $\begin{array}{l}46.28 \\
(30.8)\end{array}$ & $\begin{array}{l}39.61 \\
(19.5)\end{array}$ & $\begin{array}{l}11.73 \text { (6.21 to } \\
17.25) ; 0.000^{* *}\end{array}$ & $\begin{array}{l}3.87(-1.60 \text { to } \\
9.24) ; 0.270\end{array}$ & $\begin{array}{l}7.86 \text { (2.18 to } \\
13.54) ; 0.003^{* *}\end{array}$ & \\
\hline & $\begin{array}{l}9 \text { months } \\
\text { (t2) }\end{array}$ & $\begin{array}{l}48.82 \\
(23.8)\end{array}$ & $\begin{array}{l}45.34 \\
(31.3)\end{array}$ & $\begin{array}{l}42.40 \\
(18.9)\end{array}$ & $\begin{array}{l}10.59 \text { (4.94 to } \\
16.25) ; 0.000^{* *}\end{array}$ & $\begin{array}{l}1.19(-4.38 \text { to } \\
6.76) ; 1.000\end{array}$ & $\begin{array}{l}9.41 \text { (3.70 to } \\
15.11) ; 0.000^{* *}\end{array}$ & \\
\hline
\end{tabular}

A Adjusted for baseline physical activity and wear time

B 2-level random intercept (repeated measurements)

${ }^{* *} p<0.01$

\section{Secondary outcomes}

For all secondary outcome measures, a two level hierarchical model was applied. Table 4 shows that in general and exercise self-efficacy, no significant differences were observed. After 9 months, participants in group 2 (SSP) did score significantly higher for the physical component of the quality of life scale than participants in groups 1 (tool + SSP) and 3 (care as usual). At the end of the intervention ( 6 months), participants in both intervention groups did score significantly higher on the mental component scale compared to the care as usual group.

\section{Per protocol analyses}

The results from 174 participants (Figure 3) were analysed for the per protocol analysis. All per protocol analysis confirmed the intention to treat analysis.

\section{Subgroup analyses}

No differences were observed in outcomes for people with COPD or type 2 diabetes (results not presented). 
Table 4 Multilevel analyses for differences between the three groups for secondary outcome measures.

\begin{tabular}{|c|c|c|c|c|c|c|c|}
\hline & \multicolumn{4}{|c|}{ Unadjusted mean (SD) } & \multicolumn{3}{|c|}{ Adjusted mean difference $95 \% \mathrm{Cl}, \mathrm{p}$-value $\mathrm{A}^{\mathrm{A}}$} \\
\hline & Time points & Tool \& SSP & SSP & CAU & $\begin{array}{c}\text { Tool \& SSP - } \\
\text { CAU }\end{array}$ & $\begin{array}{l}\mathrm{SSP}- \\
\mathrm{CAU}\end{array}$ & $\begin{array}{c}\text { Tool \& SSP - } \\
\text { SSP }\end{array}$ \\
\hline \multicolumn{8}{|l|}{ Self-efficacy } \\
\hline \multirow[t]{3}{*}{$\begin{array}{l}\text { General self- } \\
\text { efficacy scale }\end{array}$} & $\begin{array}{l}\text { Baseline } \\
\text { (t0) }\end{array}$ & $\begin{array}{l}3.2 \\
(0.5)\end{array}$ & $\begin{array}{l}3.2 \\
(0.5)\end{array}$ & $\begin{array}{l}3.1 \\
(0.5)\end{array}$ & $\begin{array}{l}0.03(-0.10 \text { to } \\
0.16) ; 1.000\end{array}$ & $\begin{array}{l}0.03(0.10 \text { to } \\
0.16) ; 1.000\end{array}$ & $\begin{array}{l}-0.00(-0.13 \text { to } \\
0.13) ; 1.000\end{array}$ \\
\hline & $\begin{array}{l}\text { 4-6 months } \\
\text { (t1) }\end{array}$ & $\begin{array}{l}3.3 \\
(0.4)\end{array}$ & $\begin{array}{l}3.3 \\
(0.5)\end{array}$ & $\begin{array}{l}3.2 \\
(0.4)\end{array}$ & $\begin{array}{l}0.05(-0.09 \text { to } \\
0.18) ; 1.000\end{array}$ & $\begin{array}{l}0.02(-0.11 \text { to } \\
0.15) ; 1.000\end{array}$ & $\begin{array}{l}0.03(-0.10 \text { to } \\
0.16) ; 1.000\end{array}$ \\
\hline & $\begin{array}{l}9 \text { months } \\
\text { (t2) }\end{array}$ & $\begin{array}{l}3.2 \\
(0.5)\end{array}$ & $\begin{array}{l}3.3 \\
(0.5)\end{array}$ & $\begin{array}{l}3.2 \\
(0.4)\end{array}$ & $\begin{array}{l}0.01(-0.13 \text { to } \\
0.15) ; 1.000\end{array}$ & $\begin{array}{l}0.00(-0.13 \text { to } \\
0.13) ; 1.000\end{array}$ & $\begin{array}{l}0.01(-0.13 \text { to } \\
0.14) ; 1.000\end{array}$ \\
\hline \multirow[t]{3}{*}{$\begin{array}{l}\text { Exercise self- } \\
\text { efficacy scale }\end{array}$} & $\begin{array}{l}\text { Baseline } \\
\text { (to) }\end{array}$ & $\begin{array}{l}55.4 \\
(17.0)\end{array}$ & $\begin{array}{l}53.1 \\
(21.3)\end{array}$ & $\begin{array}{l}54.0 \\
(19.2)\end{array}$ & $\begin{array}{l}1.10(-5.04 \text { to } \\
10.38) ; 1.000\end{array}$ & $\begin{array}{l}-0.68(-8.36 \text { to } \\
7.01) ; 1.000\end{array}$ & $\begin{array}{l}2.67(-5.04 \text { to } \\
10.38) ; 1.000\end{array}$ \\
\hline & $\begin{array}{l}\text { 4-6 months } \\
\text { (t1) }\end{array}$ & $\begin{array}{l}59.7 \\
(17.3)\end{array}$ & $\begin{array}{l}59.7 \\
(19.6)\end{array}$ & $\begin{array}{l}54.5 \\
(17.4)\end{array}$ & $\begin{array}{l}4.86(-3.12 \text { to } \\
12.83) ; 0.431\end{array}$ & $\begin{array}{l}5.41(-2.52 \text { to } \\
13.35) ; 0.304\end{array}$ & $\begin{array}{l}-0.56(-8.61 \text { to } \\
7.50) ; 1.000\end{array}$ \\
\hline & $\begin{array}{l}9 \text { months } \\
(\mathrm{t} 2)\end{array}$ & $\begin{array}{l}52.1 \\
(16.1)\end{array}$ & $\begin{array}{l}60.3 \\
(19.1)\end{array}$ & $\begin{array}{l}56.5 \\
(19.2)\end{array}$ & $\begin{array}{l}-0.03(-8.01 \text { to } \\
7.94) ; 1.000\end{array}$ & $\begin{array}{l}3.60(-4.33 \text { to } \\
11.53) ; 0.828\end{array}$ & $\begin{array}{l}-3.63(-11.69 \text { to } \\
4.43) ; 0.838\end{array}$ \\
\hline \multicolumn{8}{|l|}{ Quality of life } \\
\hline \multirow[t]{3}{*}{$\begin{array}{l}\text { RAND physical } \\
\text { component }^{D}\end{array}$} & $\begin{array}{l}\text { Baseline } \\
\text { (t0) }\end{array}$ & $\begin{array}{l}42.5 \\
(11.1)\end{array}$ & $\begin{array}{l}46.1 \\
(9.8)\end{array}$ & $\begin{array}{l}45.8 \\
(9.4)\end{array}$ & $\begin{array}{l}-0.31(-2.48 \text { to } \\
1.86) ; 1.000\end{array}$ & $\begin{array}{l}0.20(-1.96 \text { to } \\
2.35) ; 1.000\end{array}$ & $\begin{array}{l}-0.51(-2.69 \text { to } \\
1.68) ; 1.000\end{array}$ \\
\hline & $\begin{array}{l}\text { 4-6 months } \\
\text { (t1) }\end{array}$ & $\begin{array}{l}45.2 \\
(9.5)\end{array}$ & $\begin{array}{l}46.8 \\
(10.0)\end{array}$ & $\begin{array}{l}47.0 \\
(10.0)\end{array}$ & $\begin{array}{l}-0.07(-2.32 \text { to } \\
2.19) ; 1.000\end{array}$ & $\begin{array}{l}-0.08(-2.33 \text { to } \\
2.17) ; 1.000\end{array}$ & $\begin{array}{l}0.01(-2.30 \text { to } \\
2.33) ; 1.000\end{array}$ \\
\hline & $\begin{array}{l}9 \text { months } \\
\text { (t2) }\end{array}$ & $\begin{array}{l}44.1 \\
(9.5)\end{array}$ & $\begin{array}{l}48.2 \\
(8.6)\end{array}$ & $\begin{array}{l}45.8 \\
(9.5)\end{array}$ & $\begin{array}{l}0.34(-1.96 \text { to } \\
2.64) ; 1.000\end{array}$ & $\begin{array}{l}2.99(0.72 \text { to } \\
5.26) ; 0.005^{* *}\end{array}$ & $\begin{array}{l}-2.65(-4.99 \text { to } \\
0.32) ; 0.020^{*}\end{array}$ \\
\hline \multirow[t]{3}{*}{$\begin{array}{l}\text { RAND Mental } \\
\text { component }^{\mathrm{D}}\end{array}$} & $\begin{array}{l}\text { Baseline } \\
\text { (to) }\end{array}$ & $\begin{array}{l}48.2 \\
(10.3)\end{array}$ & $\begin{array}{l}48.6 \\
(11.7)\end{array}$ & $\begin{array}{l}50.1 \\
(9.5)\end{array}$ & $\begin{array}{l}-0.30(-3.27 \text { to } \\
2.68) ; 1.000\end{array}$ & $\begin{array}{l}-0.39(-3.34 \text { to } \\
2.56) ; 1.000\end{array}$ & $\begin{array}{l}0.09(-2.90 \text { to } \\
3.09) ; 1.000\end{array}$ \\
\hline & $\begin{array}{l}\text { 4-6 months } \\
\text { (t1) }\end{array}$ & $\begin{array}{l}48.8 \\
(10.6)\end{array}$ & $\begin{array}{l}51.6 \\
(11.3)\end{array}$ & $\begin{array}{l}47.7 \\
(9.8)\end{array}$ & $\begin{array}{l}3.23(0.14 \text { to } \\
6.32) ; 0.04^{*}\end{array}$ & $\begin{array}{l}4.39 \text { (1.32 to } \\
7.47) ; 0.002^{* *}\end{array}$ & $\begin{array}{l}-1.16(-4.33 \text { to } \\
2.01) ; 1.000\end{array}$ \\
\hline & $\begin{array}{l}9 \text { months } \\
\text { (t2) }\end{array}$ & $\begin{array}{l}48.3 \\
(11.7)\end{array}$ & $\begin{array}{l}50.1 \\
(10.9)\end{array}$ & $\begin{array}{l}50.3 \\
(8.3)\end{array}$ & $\begin{array}{l}0.21(-2.94 \text { to } \\
3.36) ; 1.000\end{array}$ & $\begin{array}{l}0.23(-2.88 \text { to } \\
3.34) ; 1.000\end{array}$ & $\begin{array}{l}-0.02(-3.22 \text { to } \\
3.17) ; 1.000\end{array}$ \\
\hline
\end{tabular}

A Linear mixed model 2-level random intercept (repeated measurements)

B Adjusted for baseline General self-efficacy scale, computer use, and baseline physical activity moderate + vigorous

CAdjusted for baseline Exercise self-efficacy scale

$D$ Adjusted for baseline RAND physical component and baseline RAND mental component

* $P<0.05$

$* * P<0.01$ 


\section{Discussion}

\section{Principal findings}

The complete It's LiFe! intervention led to significant improvement of moderate to vigorous physical activity among patients with COPD or type 2 diabetes between 40 and 70 years old in primary care, compared to usual care. Right after the intervention period, the entire intervention added 12 minutes per day of moderate to vigorous physical activity compared to care as usual. Three months after the intervention period, this progress was still significant (11 minutes). This study also proved that use of the tool on top of the SSP is more effective than the SSP only. The added value of the tool was an additional 8 minutes of moderate to vigorous physical activity per day. The SSP alone had no significant effect on physical activity compared to care as usual. For the secondary outcome measures, the intervention effect was not evident. It did not result in higher self-efficacy levels. Only the scores on the mental component scale of quality of life showed higher levels directly after both interventions, compared to care as usual, but this difference was not maintained after nine months. At nine months follow up, participants in the SSP group scored significantly higher on the physical component of the quality of life scale compared to the other groups.

\section{Strengths and limitations}

To the best of our knowledge, this is the first randomised controlled trial that tests the added value of a monitoring and feedback tool in addition to counselling by the PN. An important strength of this study is the objective measurement of the primary outcome measure-physical activity - by an activity monitor instead of a subjective questionnaire. Other strengths are randomisation at the practice level to minimise contamination, delay of randomisation until after inclusion of the participants, the minimisation of Hawthorne effects by avoiding contacts between the researchers and participants; and simultaneous with the effect study, a process evaluation was conducted. The latter revealed that despite technical difficulties, the intervention was carried out as intended by the PNs. Another strength of this study is the pragmatic approach. Since the interventions were adapted and embedded in care as usual, it is more likely that the effects will be sustained in the daily primary care setting. ${ }^{40}$

Limitations of this study were that the mean baseline physical activity was above the recommended level of 30 minutes of moderate to vigorous activity a day, only $10 \%$ of the approached family practices agreed to participate in the study and only $37 \%$ of the approached patients agreed to participate in the intervention. These factors may have induced a selection bias, which makes the results less generalizable. However, a common reason for family practices to refuse participation was the required time investment for the practice nurse. Part of the time investment was for research purposes, which will 
be eliminated, if embedded in daily practice. The low reach among patients may be explained by the fact that in this study patients with low physical activity levels who were not aware of the problem of their inactivity (according to the transtheoretical model of behaviour change, ${ }^{41}$ the precontemplation phase of change) were not included, because the decision to participate had to be made before the consultation with the PN to create awareness could have taken place. In daily practice, the PN starts with raising awareness in regular consultations, which may result in a shift to the contemplation or preparation phase of change, and after this, patients will be asked if they are willing to work on their lifestyle with the help of the It's LiFe! intervention. Another limitation of this study was that cycling, swimming, strength training, and all upper body movements were not taken into account in the primary outcome measure because these could not be captured with the Pam. Furthermore, the follow-up was relatively short, three months after the intervention period. Ideally, a 12 month follow-up is recommended. ${ }^{42}$ Due to time constraints, this was not possible. Clinical outcomes were not measured to avoid the Hawthorne effect in the care as usual group.

\section{Comparison with prior work}

From the result that the tool embedded in the SSP is effective in contrast to the SSP alone, it can be concluded that the automated self-monitoring and feedback component and/or the fact that the PN could see the objective measured PA results, was the most powerful element of the combined intervention. This is in line with the conclusion of a meta-analysis, that PA intervention studies for chronically ill patients incorporating selfmonitoring showed a greater effect than studies without self-monitoring. ${ }^{43}$ In the SSP, participants only monitored their behaviour during the first two weeks by using an activity diary. The fact that PA was measured objectively in group 1 may also have reinforced the goal setting component. Goal setting is more effective if goals are set with a specific outcome, proximal in terms of attainment, and realistic for the individual. ${ }^{13}$ This is easier to achieve if objective PA results are available for the patient and the PN, and goals can be adapted during the intervention period based on the obtained results. The individual effect of the tool without the guidance by the PN cannot be extracted from this research, although we do expect that guidance by the nurse is an essential element of the intervention for first raising awareness, risk communication, social support, perseverance with the intervention, and adoption and persistent use of the tool. From the pilot study, it was learned that participants felt a desire to succeed due to the commitment they made with the PN and the effort she put into them. ${ }^{21}$ Other research also showed the importance of professional advice and guidance with continued support for the improvement of physical activity levels. ${ }^{12}$

Other studies demonstrated that a reduction in the number of contacts diminished the behaviour change that had been already achieved, especially when the intervention 
ends. ${ }^{13,44,45}$ In this study, three months after the intervention period, group 1 was still significantly more active than the care as usual and the SSP group.

Although exercise self-efficacy is positively correlated with physical activity levels, ${ }^{34}$ no significant differences were found on this scale between the groups, nor on general self-efficacy. This is in line with the findings from the It's LiFe! pilot study. ${ }^{21}$ Surprisingly, no effects were found on the physical component of the quality of life scale directly after the intervention, but it did improve in the SSP group three months after the intervention. We have no explanation for this observation. Awareness that physical activity is being monitored might influence habitual behaviour. ${ }^{46}$ For the intervention, this was a desirable effect of the It's LiFe! tool. However, it was an undesired effect of the use of the Pam. In this view, the proven effectiveness of the total intervention on the primary outcome-moderate to vigorous physical activity-is even more distinct considering the fact that those participating in research often show social desirable behaviour while wearing an accelerometer for a short period of the time. ${ }^{47}$ Participants in group 1, however, became used to being observed with an accelerometer for four and six months, which could have led to less social desirable behaviours during the research measurement periods, compared to the other groups.

\section{Implications for practice and future research}

Results of this study revealed the powerful addition of continuous support by the use of a monitoring and feedback tool in addition to behaviour change counselling. Because of this added value, it seems worthwhile to implement the intervention on a larger scale. However, cost-effectiveness should be investigated. To encourage general practices to adopt this intervention, health insurance companies should stimulate self-management support regarding physical activity with financial reimbursements for general practices. The fact that the availability and use of smartphones and wearables to measure physical activity is growing ${ }^{48}$ is promising for the adoption of the intervention. In daily practice, the intervention can be easily tailored to the individual needs of the patient-for example, more time for raising awareness, or referral to an exercise programme with a physiotherapist if exercise self-efficacy or capacity is considered too low. In addition, the intervention can be more extensive or recurrent in care as usual with more emphasis on habit formation, instead of a determined period of four to six months. The application of this intervention to other target groups should be investigated just as the execution by other care providers as physiotherapists and dieticians.

\section{Conclusions}

The monitoring and feedback tool, if embedded into a counselling protocol, was an effective instrument to improve physical activity of patients with COPD or type 2 diabetes between 40 and 70 years old. This improvement was sustained for 3 months. 
Counselling without the tool was not effective. The use of technology added to counselling is promising for physical activity behaviour change. Future research about the cost-effectiveness and application under more tailored conditions and in other target groups is recommended.

\section{Acknowledgements}

The project is funded by ZonMw (The Netherlands Organization for Health Research and Development) in the program, "New Instruments for Healthcare", and partly by the insurance company $\mathrm{CZ}$. The companies involved in the development of the tool were IDEE Maastricht (the Netherlands); Maastricht Instruments Ltd. (the Netherlands); and Sananet Care Ltd. (the Netherlands). During the trial, Sananet Care Ltd. manned the helpdesk and Maastricht Instruments Ltd. sent new tools to the participants if technical problems arose.

The authors would like to thank the participants in the study: the patients, practice nurses and GPs, and the patient representatives, Jos Donkers and Ina van Opstal. Furthermore, we would like to acknowledge April Boessen for her help with the data entry, Claudia Valentijn for her help at the service desk, and Willem Bekker for the $100 \%$ physical activity data check.

\section{Conflicts of Interest}

A grant from ZonMw and from Insurance Company CZ was received during the conduct of the study.

\section{Abbreviations}

COPD: chronic obstructive pulmonary disease

DM2: type 2 diabetes

GP: general practitioner

It's LiFe!: Interactive Tool for Self-management through Lifestyle Feedback

METS: metabolic equivalents

ML: maximum likelihood

PA: physical activity

Pam: Physical Activity Monitor AM300

PN: practice nurse

REML: restricted maximum likelihood

SSP: self-management support programme 


\section{References}

1. Lee IM, Shiroma EJ, Lobelo F, Puska P, Blair SN, Katzmarzyk PT. Effect of physical inactivity on major noncommunicable diseases worldwide: an analysis of burden of disease and life expectancy. Lancet 2012;380(9838):219-29.

2. Blair SN. Physical inactivity: the biggest public health problem of the 21st century. Br J Sports Med 2009;43(1):1-2.

3. Warburton DE, Nicol CW, Bredin SS. Health benefits of physical activity: the evidence. CMAJ 2006;174(6):801-9.

4. Hallal PC, Andersen LB, Bull FC, Guthold R, Haskell W, Ekelund U. Global physical activity levels: surveillance progress, pitfalls, and prospects. Lancet 2012;380(9838):247-57.

5. World Health Organization. Physical Inactivity: A Global Public Health Problem. Global Strategy on Diet, Physical Activity and Health.

http://www.who.int/dietphysicalactivity/factsheet_inactivity/en/\#.Ug4iwM91cg8.email. Last Accessed March 24, 2015.

6. NCD prevention: investments [corrected] that work for physical activity. Br J Sports Med 2012;46(10):70912.

7. Peterson JA. Get moving! Physical activity counseling in primary care. J Am Acad Nurse Pract 2007;19(7):349-57.

8. Heiligers PJM, Noordman, J., Korevaar, J.C., Dorsman, S., Hingstman, L., Dulmen, A.M. van, Bakker, D.H. de. Kennisvraag: praktijkondersteuners in de huisartspraktijk (POH's) klaar voor de toekomst? Utrecht: Nivel, 2012.

9. Lawlor DA, Hanratty B. The effect of physical activity advice given in routine primary care consultations: a systematic review. J Public Health Med 2001;23(3):219-26.

10. Avery L, Flynn D, van Wersch A, Sniehotta FF, Trenell MI. Changing physical activity behavior in type 2 diabetes: a systematic review and meta-analysis of behavioral interventions. Diabetes care 2012;35(12):2681-9.

11. van Achterberg T, Huisman-de Waal GG, Ketelaar NA, Oostendorp RA, Jacobs JE, Wollersheim HC. How to promote healthy behaviours in patients? An overview of evidence for behaviour change techniques. Health Promot Int 2011;26(2):148-62.

12. Hillsdon M, Foster C, Thorogood M. Interventions for promoting physical activity. Cochrane Database Syst Rev 2005(1):CD003180.

13. Artinian NT, Fletcher GF, Mozaffarian D, Kris-Etherton P, Van Horn L, Lichtenstein AH, et al. Interventions to promote physical activity and dietary lifestyle changes for cardiovascular risk factor reduction in adults: a scientific statement from the American Heart Association. Circulation 2010;122(4):406-41.

14. Cowan LT, Van Wagenen SA, Brown BA, Hedin RJ, Seino-Stephan Y, Hall PC, et al. Apps of steel: are exercise apps providing consumers with realistic expectations?: a content analysis of exercise apps for presence of behavior change theory. Health Educ Behav 2013;40(2):133-9.

15. Bort-Roig J, Gilson ND, Puig-Ribera A, Contreras RS, Trost SG. Measuring and influencing physical activity with smartphone technology: a systematic review. Sports Med 2014;44(5):671-86.

16. Verwey R, van der Weegen S, Spreeuwenberg M, Tange H, van der Weijden T, de Witte L. A monitoring and feedback tool embedded in a counselling protocol to increase physical activity of patients with COPD or type 2 diabetes in primary care: study protocol of a three-arm cluster randomised controlled trial. BMC Fam Pract 2014;15(1):93.

17. Kemper H, Ooijendijk W, Stiggelbout M. Consensus over de Nederlandse norm voor gezond bewegen. TSG-Tijdschrift voor Gezondheidswetenschappen 2000;78(3):180-83.

18. Verwey R, van der Weegen S, Spreeuwenberg M, Tange H, van der Weijden T, de Witte L. Upgrading physical activity counselling in primary care in the Netherlands: The systematic development of a selfmanagement support programme combined with mobile technology. Health Promot Int 2014 Dec 23. doi: 10.1093/heapro/dau107 
19. van der Weegen S, Verwey R, Spreeuwenberg M, Tange H, van der Weijden T, de Witte L. The Development of a Mobile Monitoring and Feedback Tool to Stimulate Physical Activity of People with a Chronic Disease in Primary Care: A User-Centered Design. JMIR Mhealth Uhealth 2013;1(2):e8.

20. van der Weegen S, Verwey R, Tange HJ, Spreeuwenberg MD, de Witte LP. Usability testing of a monitoring and feedback tool to stimulate physical activity. Patient Prefer Adherence 2014;8:311-22.

21. Verwey R, van der Weegen S, Spreeuwenberg M, Tange H, van der Weijden T, de Witte L. A pilot study of a tool to stimulate physical activity in patients with COPD or type 2 diabetes in primary care. $J$ Telemed Telecare 2014;20(1):29-34.

22. Verwey R, van der Weegen S, Tange H, Spreeuwenberg M, van der Weijden T, de Witte L. Get moving: the practice nurse is watching you! A case study of the user-centred design process and testing of a webbased coaching system to stimulate the physical activity of chronically ill patients in primary care. Inform Prim Care 2012;20(4):289-98.

23. Wendel-Vos GC, Schuit AJ, Saris WH, Kromhout D. Reproducibility and relative validity of the short questionnaire to assess health-enhancing physical activity. J Clin Epidemio/ 2003;56(12):1163-9.

24. NISB. Sportief bewegen met een chronische longaandoening. 2012 Archived at: http://www. webcitation.org/6PDsk5Av1.

25. NISB. Sportief bewegen met diabetes mellitus. 2012. Archived at: http://www.webcitation.org/6PDsgeCvk.

26. NISB. 30 Beweegtips. 30 minuten bewegen. 2012. Archived at: http://www.webcitation.org/6PDsoFdJt.

27. Glasgow RE, Emont S, Miller DC. Assessing delivery of the five 'As' for patient-centered counseling. Health Promot Int 2006;21(3):245-55.

28. Whitlock EP, Orleans CT, Pender N, Allan J. Evaluating primary care behavioral counseling interventions: an evidence-based approach. Am J Prev Med 2002;22(4):267-84.

29. Damen E. PAM. Secondary PAM 2014. http://www.pam.com/index.php?pid=. Last Accessed March 24, 2015. Archived at: http://www.webcitation.org/6PEz8ql7Y.

30. Slootmaker SM, Chin APMJ, Schuit AJ, van Mechelen W, Koppes LL. Concurrent validity of the PAM accelerometer relative to the MTI Actigraph using oxygen consumption as a reference. Scand J Med Sci Sports 2009;19(1):36-43.

31. Vooijs M, Alpay LL, Snoeck-Stroband JB, Beerthuizen T, Siemonsma PC, Abbink JJ, et al. Validity and usability of low-cost accelerometers for internet-based self-monitoring of physical activity in patients with chronic obstructive pulmonary disease. Interact J Med Res 2014;3(4):e14.

32. World Health Organization. Global Recommendations on Physical Activity for Health. 2011. http://www. who.int/dietphysicalactivity/physical-activity-recommendations-18-64years.pdf?ua=1 Accessed March 24, 2015. Archived at: http://www.webcitation.org/6PF1UBuEa.

33. Schwarzer R, Jerusalem M. Generalized Self-Efficacy scale. In: Weinman J, Wright S, Johnston M. Measures in health psychology: a user's portfolio. Causal and control Beliefs. Windsor: NFER-NELSON, 1995:35-37.

34. van der Heijden MM, Pouwer F, Pop VJ. Psychometric Properties of the Exercise Self-efficacy Scale in Dutch Primary Care Patients with Type 2 Diabetes Mellitus. Int J Behav Med 2014;21(2):394-401.

35. Shin Y, Jang H, Pender NJ. Psychometric evaluation of the exercise self-efficacy scale among Korean adults with chronic diseases. Res Nurs Health 2001;24(1):68-76.

36. Everett B, Salamonson Y, Davidson PM. Bandura's exercise self-efficacy scale: validation in an Australian cardiac rehabilitation setting. Int J Nurs Stud 2009;46(6):824-9.

37. Van der Zee K, Sanderman R. RAND-36. Vol 1. Groningen: Northern Centre for Health Care Research, University of Groningen, the Netherlands 1993;28.

38. Ware JE, Kosinski M, Keller S. SF-36 physical and mental health summary scales: a user's manual: Health Assessment Lab., 1994.

39. Twisk JWR. Applied multilevel analysis : a practical guide. Cambridge, UK; New York: Cambridge University Press, 2006.

40. Linmans JJ, Viechtbauer W, Koppenaal T, Spigt M, Knottnerus JA. Using electronic medical records analysis to investigate the effectiveness of lifestyle programs in real-world primary care is challenging: a case study in diabetes mellitus. J Clin Epidemiol 2012;65(7):785-92. 
41. Prochaska JO, Velicer WF. The transtheoretical model of health behavior change. Am J Health Promot 1997;12(1):38-48.

42. Foster C, Richards J, Thorogood M, Hillsdon M. Remote and web 2.0 interventions for promoting physical activity. Cochrane Database Syst Rev 2013;9:CD010395.

43. Conn VS, Hafdahl AR, Brown SA, Brown LM. Meta-analysis of patient education interventions to increase physical activity among chronically ill adults. Patient Educ Couns 2008;70(2):157-72.

44. Yeh MC, Rodriguez E, Nawaz H, Gonzalez M, Nakamoto D, Katz DL. Technical skills for weight loss: 2-y follow-up results of a randomized trial. Int J Obes Relat Metab Disord 2003;27(12):1500-6.

45. Browning C, Thomas SA. Behavioural change: an evidence-based handbook for social and public health. Edinburgh; New York: Elsevier/Churchill Livingstone, 2005.

46. Pedisic Z, Bauman A. Accelerometer-based measures in physical activity surveillance: current practices and issues. Br J Sports Med 2014.

47. Casaburi R. Activity monitoring in assessing activities of daily living. COPD 2007;4(3):251-5.

48. Krijgsman J, Peeters J, Burghouts A, Brabers A, de Jong J, Beenkens F, et al. Op naar meerwaarde! eHealthmonitor. Den Haag 2014. Nictiz NIVEL http://www.nictiz.nl/page/eHealth/eHealth-monitor Accessed March 24, 2015. 



\section{CHAPTER 8}

\section{Process evaluation of physical activity counselling with and without the use of mobile technology: a mixed methods study}

Submitted

Verwey R \& van der Weegen S, Spreeuwenberg $M$, Tange $H$, van der Weijden $T$, de Witte L. Process evaluation of physical activity counselling with and without the use of mobile technology: a mixed methods study 


\section{Abstract}

Background A monitoring-and-feedback tool was developed to stimulate physical activity by giving feedback on physical activity performance to patients and practice nurses. The tool consists of an activity monitor (accelerometer), wirelessly connected to a Smartphone and a web application. Use of this tool is combined with a behaviour change counselling protocol based on the Five A's model (Assess-Advise-Agree-AssistArrange).

Objectives To examine the reach, implementation and satisfaction with the counselling protocol and the tool.

Design A process evaluation was conducted in two intervention groups of a three-armed cluster RCT, in which the counselling protocol was evaluated with (group 1) and without (group 2) the use of the tool using a mixed methods design.

Settings Sixteen family practices in the South of the Netherlands.

Participants Practice nurses $(n=20)$ and their associated physically inactive patients $(n=131)$, diagnosed with COPD or type 2 diabetes, aged between $40-70$ years old, and having access to a computer with an internet connection.

Methods Semi structured interviews about the receipt of the intervention were conducted with the nurses and log files were kept regarding the consultations. After the intervention, questionnaires were presented to patients and nurses regarding compliance to and satisfaction with the intervention. Functioning and use of the tool were also evaluated by system and helpdesk logging.

Results Eighty-six percent of patients (group 1: $n=57$ and group 2: $n=56$ ) and $90 \%$ of nurses (group 1: $n=10$ and group 2: $n=9$ ) responded to the questionnaires. The execution of the intervention was adequate; in $83 \%$ (group 1: $n=52$, group 2: $n=57$ ) of the patients, the number and planning of the consultations were carried out as intended. Eighty-eight percent $(n=50)$ of the patients used the tool until the end of the intervention period. Technical problems occurred in $58 \%(n=33)$. Participants with the tool were significantly more positive about the intervention than those without the tool: patients: $\chi^{2}(2$, $N=113)=11.17, p=0.004$, and nurses: $\chi^{2}(2, N=19)=6.37, p=0.040$. Use of the tool led to greater awareness of the importance of physical activity, more discipline in carrying it out and more enjoyment.

Conclusions The interventions were adequately executed and received as planned. Patients from both groups appreciated the focus on physical activity and personal attention given by the nurse. The most appreciated aspect of the combined intervention was the tool, although technical problems frequently occurred. Patients with the tool estimated more improvement of physical activity than patients without the tool. 


\section{Background}

People who are insufficiently active have a $20 \%$ to $30 \%$ increased risk of death compared to people who engage in at least 30 minutes of moderately intense physical activity on most days of the week. ${ }^{1,2}$ Since physical inactivity is the fourth leading risk factor for global mortality, the World Health Organisation agreed on targets that include a 10\% reduced prevalence of insufficient physical activity (PA) by $2025{ }^{3}$ To achieve this goal, it is recommended that routine patients' contacts in primary care should include assessment of PA, advice on the benefits of increased levels of PA, and practical support to help patients initiate and maintain healthy behaviours. ${ }^{4-7}$ Practice nurses (PNs) have regular contacts with chronically ill patients who can benefit from an active lifestyle; therefore these contacts are an excellent opportunity for promoting physical activity. Effective interventions to stimulate PA include consultations with brief negotiation or discussion to decide on reasonable and attainable goals, targeted information, and follow-up. ${ }^{8}$ New modes to support self-management through computer or mobile phones are promising. ${ }^{9-11}$ Interventions including these technological innovations show potential to change health behaviours and to improve clinical outcomes in patients with a chronic illness.

In the project Interactive Tool for Self-management through Lifestyle Feedback! (It's LiFe!) a personalized monitoring and feedback tool (Figure 1) was developed ${ }^{12}$ and tested $^{13,14}$ according to User Centred Design, a design philosophy in which the end user's needs, wants and limitations are a focus at all stages within the design process. ${ }^{15}$ This tool aims to support patients with type 2 diabetes (DM2) or Chronic Obstructive Pulmonary Disease (COPD) in achieving a more active lifestyle. The tool consists of three elements:

1. a three-dimensional activity monitor (accelerometer) worn on the hip;

2. a smartphone application (app);

3. a web application (for both patients and nurses).

The tool is employed within a behaviour change counselling protocol which is executed by PNs, named the Self-management Support Programme (SSP). ${ }^{16}$

After a successful feasibility study of the complete intervention, ${ }^{17}$ a three armed cluster randomised controlled trial was conducted to evaluate the effect of the intervention on PA, (exercise) self-efficacy, quality of life, and patient health. A detailed study protocol of the It's LiFe! RCT was published in advance, including the process evaluation of this effect study. ${ }^{18}$ 


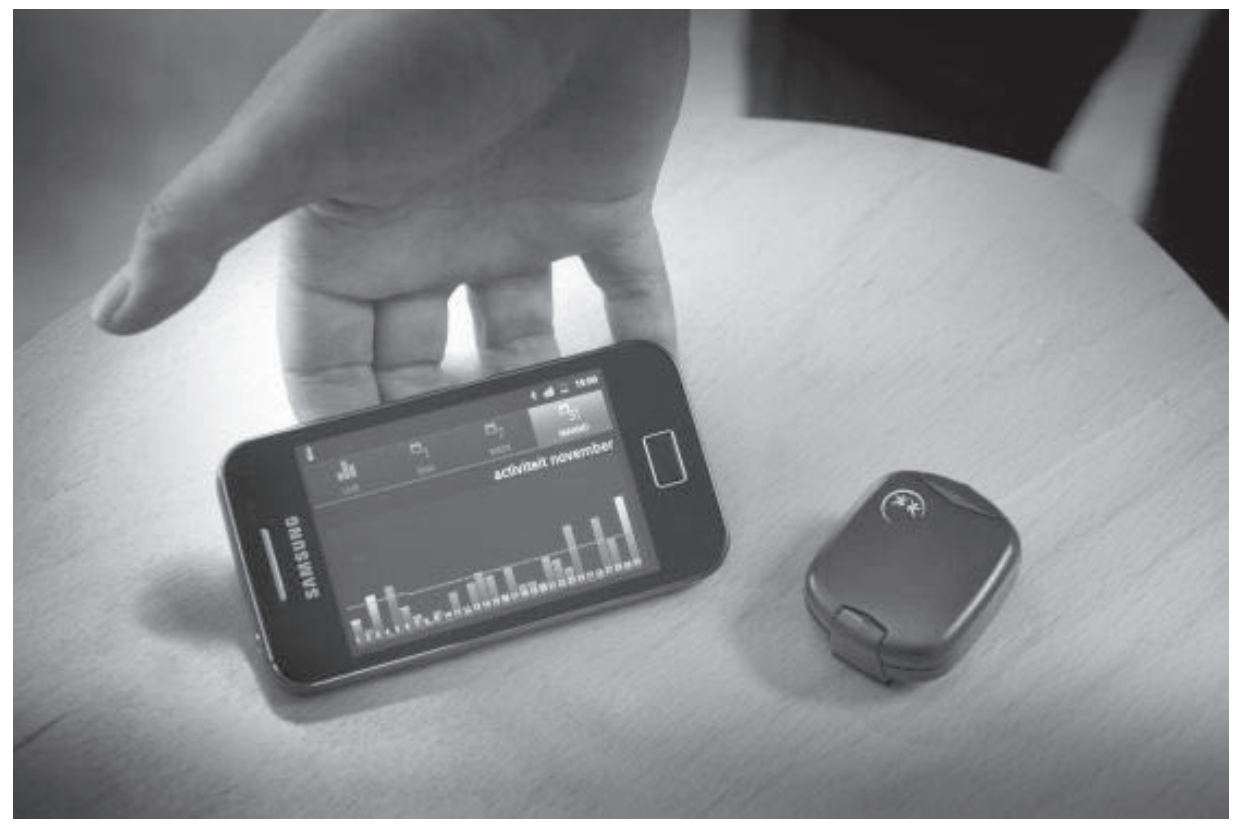

Figure 1 The It's LiFe! tool.

Incorporating a process evaluation is necessary for complex interventions to examine the context, implementation, and receipt of the intervention in depth. ${ }^{18-23}$ Process evaluations are also necessary in multi-centre trials, where 'the same' intervention may be implemented and received in different ways. ${ }^{24}$ For this study both arguments are appropriate. The intervention is complex, because it consists of a number of different aspects; both the SSP and the use of the tool, which may influence the effects of the study separately and in combination with one another. ${ }^{21}$ Furthermore, the study was set up as a multi-centre trial in which each family practice might encounter different problems with the tool and each PN following the SSP might develop her own style of coaching. The research questions of the process evaluation were:

1. Who participated in the intervention, which patients dropped out, and for what reasons?

2. To what extent was the intervention executed and received as intended?

3. How did patients and nurses experience the different aspects of the intervention (the SSP and the monitoring and feedback tool), and what suggestions did they have for improvements?

This paper presents the results of the It's LiFe! process evaluation. 


\section{Methods}

\section{Design of the process evaluation}

From December 2012 until July 2014 a process evaluation amongst participating family practices in the intervention groups of the trial was conducted. Research questions were drawn up according to the how-to guide of Saunders for developing a process-evaluation plan to assess the implementation of a targeted health promotion intervention; they focused on the following components: recruitment, reach, context, fidelity, dose delivered, dose received - exposure, and dose received - satisfaction. ${ }^{23,25}$ Table 1 shows an overview of these components, their operationalisation and corresponding measurements and timing.

\section{Setting and participants}

From June 2013 until April 2014 a three-armed cluster randomised controlled trial was conducted to measure the effects of the use of the tool embedded in a counselling protocol. The trial compared this counselling protocol with and without the use of the tool (groups 1 and 2) with usual care (group 3). A total of 24 family practices were randomly allocated into one of the three conditions: eight practices (group 1) received the complete intervention (SSP and tool), eight practices (group 2) received only the SSP, and eight practices (group 3) received care as usual. Nurses and patients randomised in the third arm, which performed and received care as usual (group 3), were not involved in the process evaluation. The intended study population for the trial consisted of 120 patients with DM2 and 120 patients with COPD.

\section{Participants and recruitment}

General practices located in southern regions of the Netherlands were invited by an invitation letter, telephone and personal contact with GP's, practice managers, and PNs, until a maximum of 24 practices was reached. In the practices that agreed to participate, the PN was asked to invite 20-32 patients (aged between 40-70 years old, and with access to the internet) who, according to the PN, did not comply with the Dutch Norm for Healthy Exercise (at least 30 minutes of moderate-intensity aerobic physical activity (equivalent to brisk walking and noticeably accelerating the heart rate on five days of the week $)^{26}$ and were sufficiently motivated to become more active. All eligible patients received an information letter with a general explanation about the trial. After randomisation on practice level, specific invitation letters were sent to these patients, according to the group their practice was assigned to, followed by a telephone call from the PN to ask the patient if he or she wanted to participate. Non-responders were asked for their reasons not to participate. Each practice was asked to include at least five patients with COPD and five patients with DM2. 
Table 1 Key components of the process evaluation

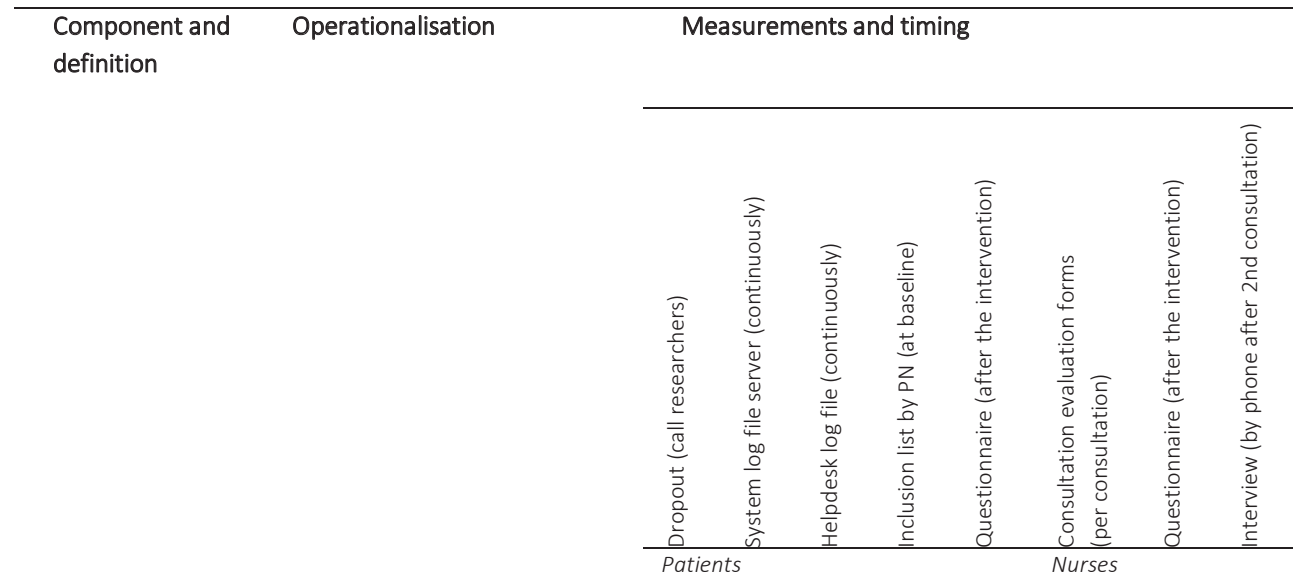

1. Who participated in the intervention, which patients dropped out, and for what reasons?

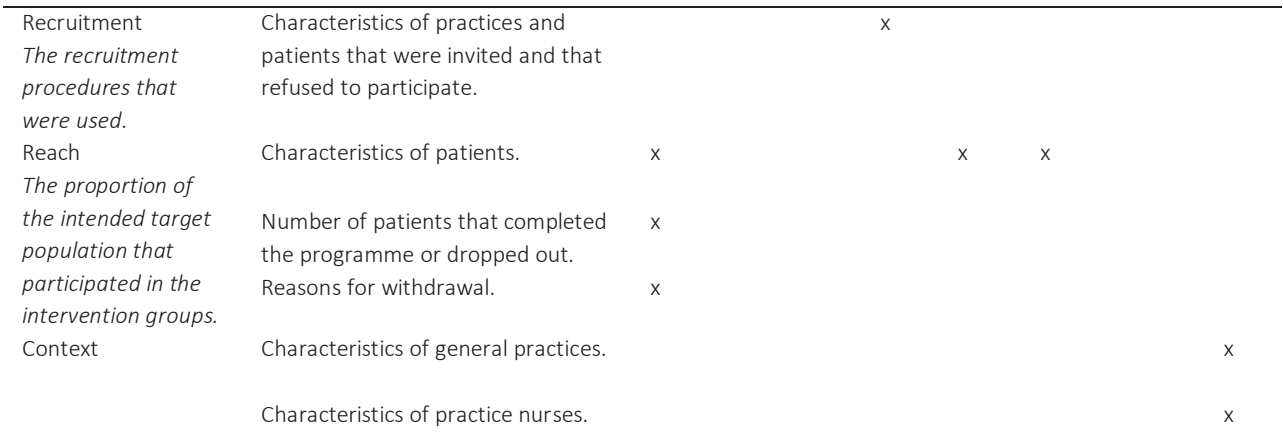

\section{To what extent was the intervention executed} and received as intended?

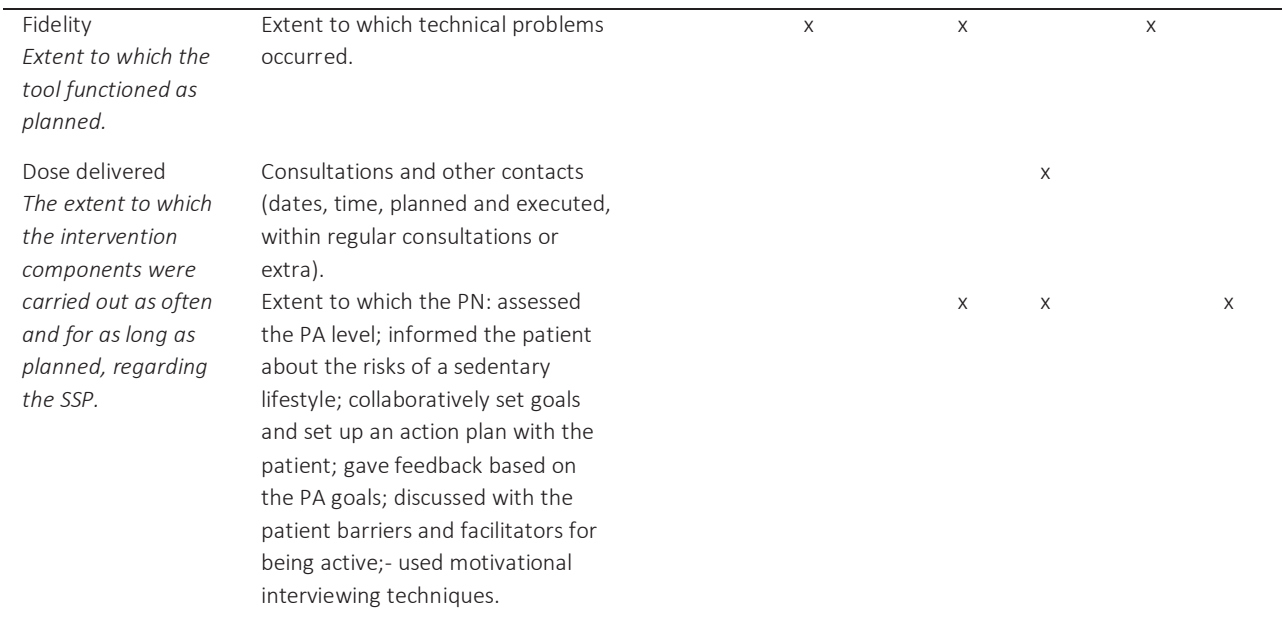




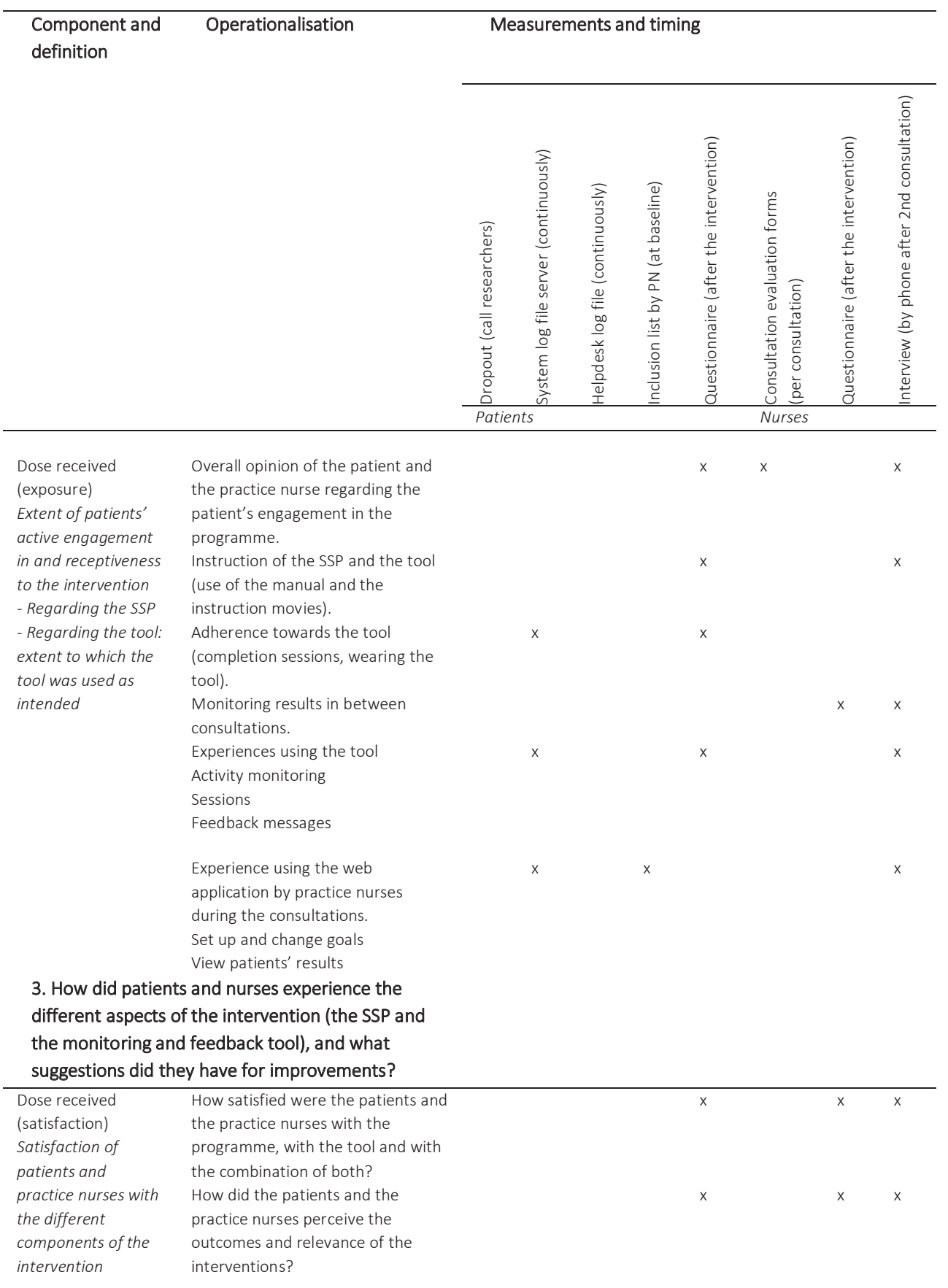




\section{Ethical approval}

The study protocol of the trial, including the process evaluation, was approved by the Medical Ethics Committee of the Maastricht University/Academic Hospital Maastricht. Written informed consent from all participating family practices and included patients was obtained.

\section{The intervention}

Patients in group 1 received the monitoring and feedback tool embedded in the SSP, whereas patients in group 2 received the same programme without the use of the tool.

Different aspects of the intervention

The different aspects of the interventions are depicted in Table 2.

The SSP

The SSP consisted of four consultations with the PN spread over a period of four to six months: in the first week, after two weeks, after two to three months and after four to six months. The consultations were based on the 'Five A's cycle' counselling technique (Assess-Advise-Agree-Assist-Arrange). ${ }^{27,28}$ Beforehand, patients received an information booklet about the course of the intervention containing the Short Questionnaire to Assess Health (SQUASH)-enhancing PA ${ }^{29}$ and a list of locally organised PA activities. In the first consultation the PN increased the awareness about the risks of physical inactivity (Advise) and the PA level of the patient was discussed using the previously completed SQUASH questionnaire (Assess). In addition, all patients received a general and a disease specific leaflet about PA (Advise). ${ }^{30,31}$ Between the first and the second consultation, patients completed an activity diary and answered questions about barriers and facilitators for PA. During the second consultation, a personal goal was set in minutes of activity per day (Agree), the nurse informed the participants about locally organised PA options (Assist), and the PN encouraged the participants to set up an activity plan to reach personal goals (Arrange). In the third consultation, possibly by mail or telephone, activity results, barriers, facilitators, and the creation of new PA habits, were discussed and some participants reconsidered their activity goal. In the last consultation, activity results, barriers, facilitators, and new habits were evaluated. ${ }^{18}$ 
Table 2 Different aspects of the intervention

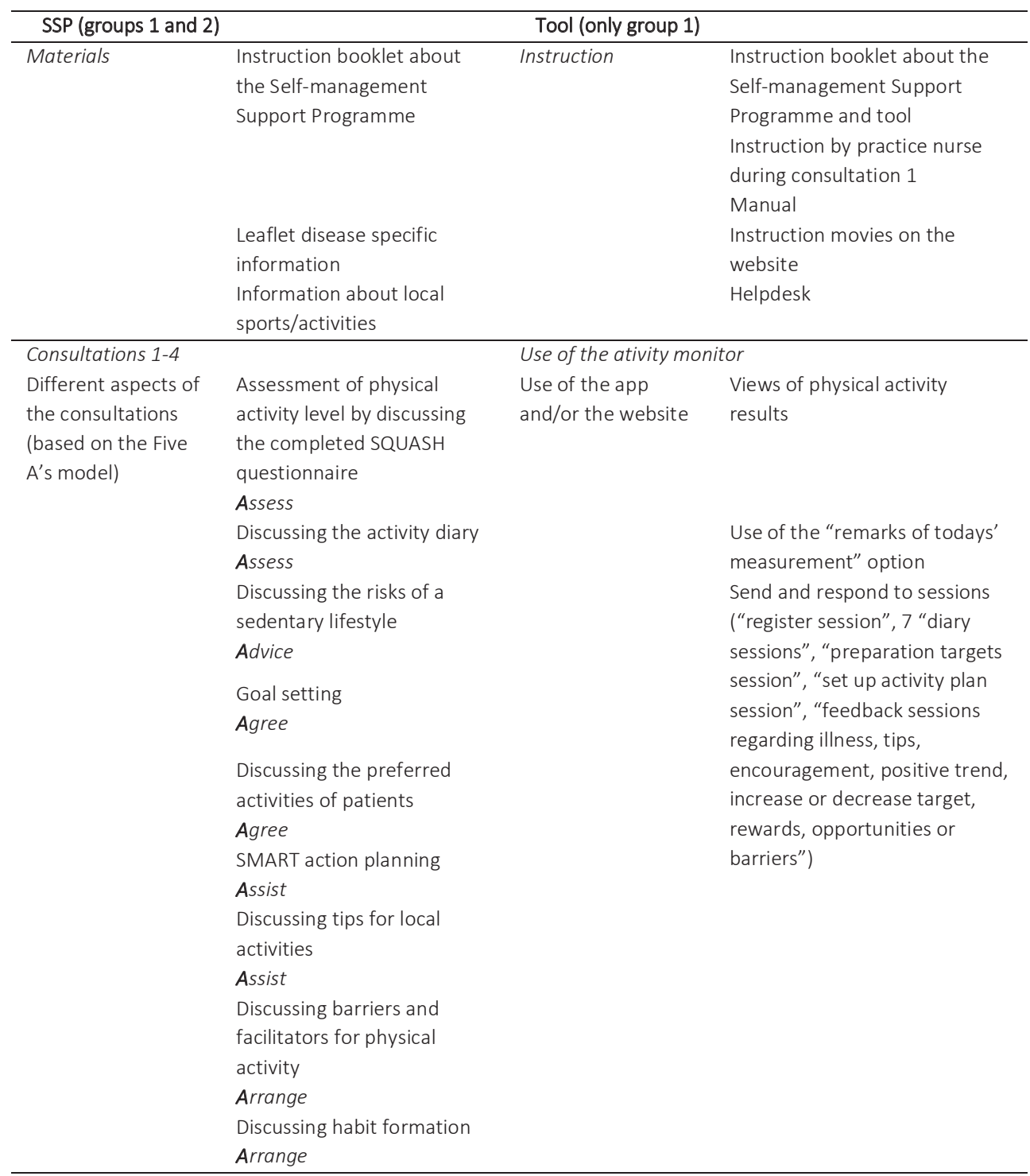

The Tool

During the first consultation the nurse provided the patients allocated in group 1 with the tool and registered the patient into the web application. Patients were asked to wear the activity monitor on a daily basis. They could see their real time activity results and history in minutes of moderate to vigorous activity on the smartphone and the web application. They were given a (data) subscription to be able to make telephone calls and to go online, with the intention that they would use the Smartphone in daily living, and consequently look at their activity results more frequently. Furthermore, they received 
dialogue sessions and - after the second consultation- feedback messages on the app on the Smartphone concerning their amount of activity in relation to their activity target. During the whole intervention, activity results and answers to dialogue sessions were visible for the PN on a secured web application and they could be used as input for the coaching in the consultations. ${ }^{17}$

\section{Acceptable delivery of the intervention}

The accomplishment of at least three out of four consultations in a period of at least three months was considered an acceptable delivery of the SSP. Consultation number 3 could also be conducted by telephone or by mail contact. PNs provided the patients with all materials during the first consultation.

Conditions for an acceptable delivery of the tool were that the tools should work according to plan, e.g. that every patient was adequately signed up, measurements were uploaded daily to the server, and that the tools were free of technical failure. To maintain these conditions, a helpdesk facility was running during working days/hours to answer technical questions, solve user problems, and replace the tool within five days, if needed.

\section{Education and training}

Patients were sent an information booklet about the course of the intervention. Further materials and instruction were given by the practice nurse.

The nurses in groups 1 and 2 were asked to watch an online web lecture and consecutively received a two-hour personal instruction at their workplace. They also received an information file with detailed instruction charts for each consultation and an explanation of the Five A's model and the intended counselling techniques.

The nurses in group 1 received a personal account, a manual, and personal instruction on how to use the web application. Through this application the nurses could monitor their patients. Furthermore, they were able to try out the tool before the start of the consultations. For technical issues they could refer to the same helpdesk as their patients.

\section{Data collection}

Both quantitative and qualitative information were collected from patients and nurses (Figure 2). The researchers developed questionnaires and interview topics by translating theoretical key elements of process evaluations ${ }^{23,25}$ into structured questions regarding the different components of the intervention. Table 1 provides a detailed overview of the data collection methods and the timing of the process evaluation. After informed consent, patient characteristics (i.e. demographics) were gathered by means of selfadministered questionnaires. The researchers collected dropout reasons throughout the intervention period by calling the patients and asking them to give reasons. 


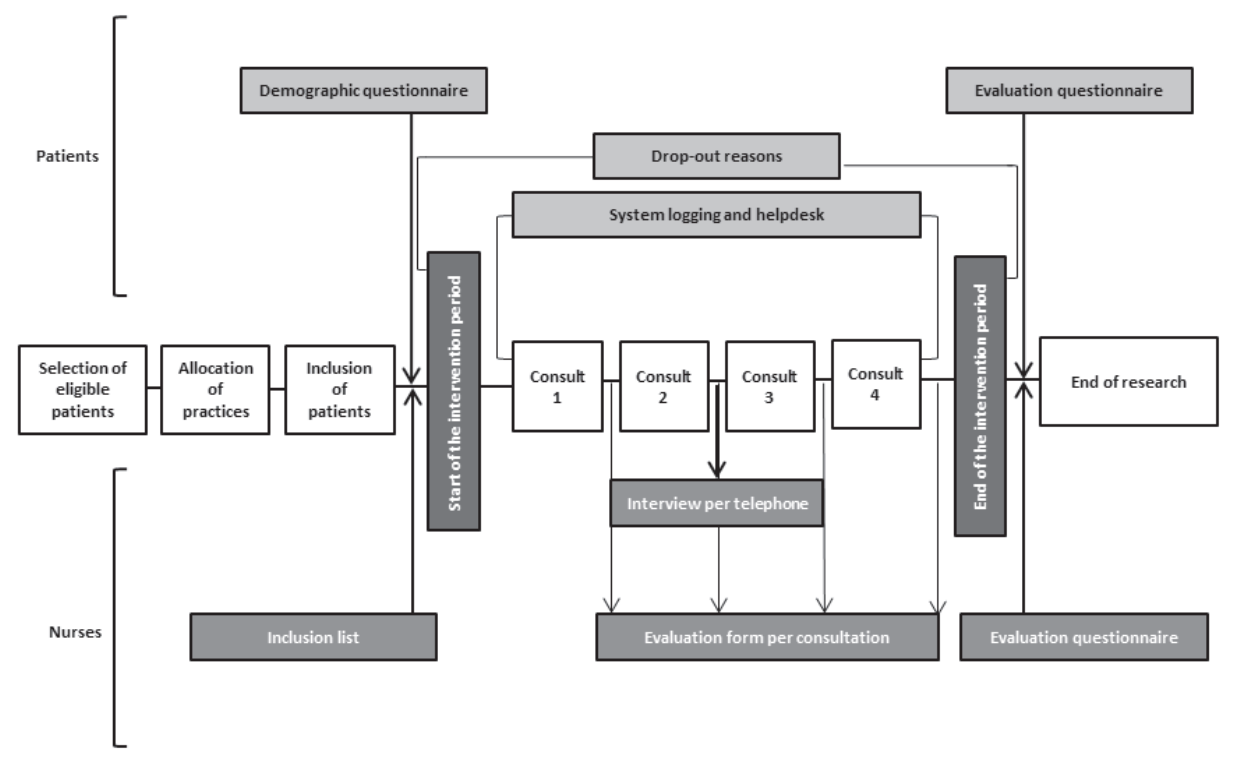

Figure 2 Data collection.

To measure exposure to the SSP, the nurses were asked to keep a record of all consultations in log files. To measure exposure to the tool, the use of the tool was measured objectively by extracting information from the server. Technical problems were logged by members of the helpdesk.

Approximately two weeks after the second consultation, all nurses of groups 1 and 2 were interviewed by telephone about their experiences so far. In the interviews, that lasted approximately half an hour, special attention was paid to the factors that might influence compliance with the intervention on two levels: complying with the advice and feedback given during the consultations, and with the monitoring and feedback tool. Directly after the intervention period, a questionnaire about their experiences and the feasibility of the intervention was sent to all nurses and patients.

To diminish Hawthorne effects, there were no direct contacts between researchers and patients. Patients were not interviewed in order to distinguish between the intervention and its evaluation. ${ }^{32}$

\section{Data analysis}

Quantitative data were analysed by means of descriptive statistics, and Fisher's exact tests were used to test if there were differences between the groups, using the IBM Statistical Product and Service Solutions (SPSS) version 22. Qualitative data (results from open questions and interviews) were analysed by two researchers (RV, SvdW) 
independently using NViVo version 9 in order to identify relevant themes. In cases of disagreement, mutual agreement was found by discussion. A concurrent triangulation strategy was applied to confirm, cross validate, and corroborate the findings. The timing of the analysis of the process data was planned before the data from the effect study were analysed, to avoid interpretation bias. ${ }^{24}$

\section{Results}

\section{Characteristics of the respondents}

One hundred and thirty-one patients were assigned to the intervention groups in 16 family practices; $86 \% \quad(n=113)$ of them responded to the process evaluation questionnaire ( $51 \%$ male, mean age 58 years SD \pm 7.7 ; group $1: n=57$ and group $2: n=56$ ). Sixty-three percent $(n=71)$ of these respondents were diagnosed with DM2 and $37 \%$ $(n=42)$ with COPD.

Of the 20 nurses (group 1: $n=11$ and group 2: $n=9$ ) who performed the intervention, 95\% responded to the questionnaire (group 1: $n=10$ and group 2: $n=9$ ) and $90 \%$ participated in the interviews (group 1: $n=9$ and group 2: $n=9$ ).

\section{Participants (recruitment, reach, and context)}

From October 2012 until May 2013 approximately 250 general practices, were invited to participate in the study. Although practices were offered an appropriate reimbursement of expenses, most practices refused because they were too busy with regular patient care.

The size of participating practices ranged from small (<90 DM2 patients) to large (>390 DM2 patients). In group 1, two practices were located in a city, in group 2 three were. In three practices in group 1 and in one practice in group 2, the intervention was carried out by two practice nurses. All nurses were female, the nurses who were interviewed had a mean age of 43.4 years (range 26-54), and their average working experience as a PN was 13.5 years (range 2-32); 11 nurses finished secondary vocational education and seven nurses higher professional education.

All nurses were instructed as planned, but the web lectures were rarely watched. All nurses were satisfied with the instruction charts per consultation. Nurses in group 1 experienced the instruction as too brief, especially to become familiar with the web application.

Nurses from the participating practices indicated that it was very difficult to find enough eligible patients, because their patient population consisted mainly of people above the age of 70 , or the patients did not master the Dutch language well enough. Before randomisation, approximately 540 patients received a general invitation letter 
and a call from their nurse, and 131 agreed to participate in the intervention groups. Reasons for not taking part in the trial were: patients were not motivated, had no time, were too busy with work, had no access to internet/computer, did not feel the need because they were physically active enough, had physical or psychosocial disabilities, or thought they were clumsy with the computer.

In both intervention groups $80 \%$ of the intended number of patients ( $n=64)$ started the intervention. In total, $17 \%$ of the patients (group 1: $n=13$, group 2: $n=9$ ) did not receive the minimal intervention as intended. The average intervention period of the remaining patients was 25 weeks. Of the 131 patients who agreed, $2 \%(n=3)$ never started the intervention, 5\% $(n=6)$ had only one consultation, $8 \%(n=11)$ had two consultations, $11 \%(n=15)$ had three consultations, and $73 \%(n=96)$ had all four consultations. Dropout reasons were technical problems with the tool $(n=8)$, becoming ill $(n=7)$, personal or family circumstances $(n=3)$, too busy with work $(n=2)$, or lack of perceived usefulness $(n=2)$.

\section{Execution of and receptiveness towards the interventions (fidelity - dose delivered - dose received (exposure))}

The first practices started with the intervention in June 2013, and the last practices finished in April 2014.

\section{Self-management Support Programme}

According to the log files per consultation, often the consultations took longer than the intended 20 minutes. This was the case with the first consultation (group 1: $77 \% \mathrm{n}=49$ and group 2: $50 \% n=33$ ), the second consultation (group 1: $40 \% n=26$ and group 2: $33 \%$ $n=21$ ), and the last consultation (group 1: $22 \% n=14$ and group 2: $52 \% n=34$ ). Thirty-six percent of the consultations were conducted within a regular consultation, whereas $64 \%$ were planned as extra. The nurses had the opportunity to contact patients in between consultations to monitor results and experiences of patients; in group 1 this happened more often $(60 \% n=6)$ than in group $2(33 \% n=3)$. In group 1 the nurses had more contact with patients in between consultations; this was mostly related to technical problems with the tool.

Regarding the execution of the Five A's model, there were no significant differences between the groups and most components were executed as planned.Table 3 shows percentages of patients who remembered the different aspects of the Five A's model. The nurses stated that, by performing the intervention, they became more conscious about the PA of patients. They also mentioned the fact that lots of patients indicated that they were already sufficiently active, and that despite the wish of patients to become more active, there were always lots of excuses for not doing so. 
Table 3 Execution of the Five A's model within the SSP according to patients

\begin{tabular}{|c|c|c|c|c|}
\hline & \multicolumn{2}{|c|}{$\begin{array}{l}\text { Group } 1 \\
n=57\end{array}$} & \multicolumn{2}{|c|}{$\begin{array}{l}\text { Group } 2 \\
n=56\end{array}$} \\
\hline & $\mathrm{n}$ & $\%$ yes $^{\mathrm{A}}$ & $\mathrm{n}$ & $\%$ yes $^{A}$ \\
\hline \multicolumn{5}{|l|}{ Assessment of PA level } \\
\hline by discussing the completed SQUASH questionnaire & 45 & $70 \%$ & 45 & $73 \%$ \\
\hline Discussing the activity diary & 49 & $63 \%$ & 46 & $64 \%$ \\
\hline Discussing the risks of a sedentary lifestyle & 55 & $91 \%$ & 49 & $80 \%$ \\
\hline Goal setting & 52 & $84 \%$ & 41 & $63 \%$ \\
\hline Discussing the preferred activities of patients & 55 & $97 \%$ & 47 & $84 \%$ \\
\hline SMART action planning & 53 & $67 \%$ & 40 & $57 \%$ \\
\hline Discussing tips for local activities & 50 & $60 \%$ & 41 & $50 \%$ \\
\hline Discussing barriers and facilitators for physical activity & 51 & $72 \%$ & 40 & $55 \%$ \\
\hline Discussing habit formation & 53 & $79 \%$ & 42 & $63 \%$ \\
\hline
\end{tabular}

All nurses agreed on the importance of self-management support, because they expect better results when patients formulate their own behaviour change goals but nurses from group 2 encountered difficulties for physical activity counselling because they had only vague ideas of physical activity levels of patients. Seventy-four percent $(n=13)$ of the nurses were positive about the SQUASH questionnaire; it gave a clear picture of patients' activities. Nurses valued talking about the risks of a sedentary lifestyle, although $22 \%$ $(n=4)$ said that for most patients this was nothing new. Some nurses found it difficult to discuss these risks in cases of severe disability $(n=2)$ or when they had already known the patient for a longer time.

Although collaborative goal setting and composing a plan of action were important parts of the intervention, some patients indicated that goals had not been discussed during the consultations. Goals were mainly set by patients themselves $(61 \% n=69)$ or in collaboration with the nurse $(32 \%, n=36)$. There was a significant difference between groups 1 and 2 in the responses regarding goal achievement; (group 1: 84\% n=48, group 2: $61 \% n=34)$ thought they had reached their goals $\chi^{2}(1, N=110)=7.50, p=0.006$. Reasons for not achieving activity goals were: physical/psychological symptoms and illness $(n=14)$, job $(n=3)$, and family problems $(n=3)$. All nurses stressed the importance of setting small achievable goals and helping patients to draw up a specific plan, but $17 \%$ $(n=3)$ of the nurses had difficulties with advising patients to plan activities in this way 
(too scholarly). More than half of the patients remembered discussing the tips for local activities; most patients simply increased their normal activities like walking and cycling.

The tool

Mobile devices (patients)

In group 1,88\% ( $n=50)$ of the patients used the tool until the end of the intervention. Reasons to stop wearing the tool were: malfunction of the tool, the belief that using it was not necessary anymore because of an appropriate activity level, or quitting the study. Only $12 \%(n=7)$ of the patients did use the phone on a daily basis for purposes other than the intervention, $56 \%(n=32)$ only occasionally, whereas $32 \%(n=18)$ never used the phone to make calls or use the internet.

Of the 64 patients who were registered in the system by the nurse, one patient did not complete the register session, due to having bad eyesight. Figure $3 \mathrm{~A}$ shows the percentages of patients who completed sessions and read feedback messages. Figure 3 $B$ shows how often different types of feedback messages were received by the patients as percentages of the total number of feedback messages $(n=1664)$.

The median of number of times that patients $(n=63)$ used the 'remarks of today's measurement' session was $30(\mathrm{IQR}=15-52)$. There were 6 diary sessions on consecutive days. On each day, $70 \%$ of the patients completed this session. The median that patients $(n=53)$ read feedback messages was $23(\mathrm{IQR}=6-35)$. The most frequently given feedback message was the request to increase the daily target goal. This message was automatically sent when a patient reached his or her current target goal for more than 10 days during the previous two weeks.

Fifty-eight percent $(n=33)$ of the patients experienced problems using the tool. During the intervention period, 190 issues were registered by the helpdesk. Most problems occurred with the connection between the sensor and the phone $(n=88)$ or the connection between the phone and the server $(n=30)$. A big issue in the beginning of the trial was that most phones were of a newer type than the one the app originally was developed for. After implementation by six patients, it appeared that those phones logged out automatically and patients had to login again every day. These phones were taken back and new phones were distributed to the practices.

Complications with the connection between the activity monitor and the phone were in some cases due to a user error or misinterpretation of the results; patients disabled the connection between sensor and phone by disconnecting Bluetooth, they did not charge the sensor correctly, or they expected more results than showed. However, in most cases it was a technical problem, and therefore a new sensor was sent or brought to the patient. Regarding the connection between the phone and the server, there were 21 login issues; patients forgot to login on the phone or experienced problems during login. Another reason for a lack of data in the monitor was disconnection of the internet connection by the patient. There were also some hardware 
problems with the activity monitor; the case broke $(n=3)$ or the clip broke $(n=3)$. Other issues ranged from a forgotten password to accidentally deleting the app and/or widget. Most registered issues that occurred were relatively easily solvable registration problems.

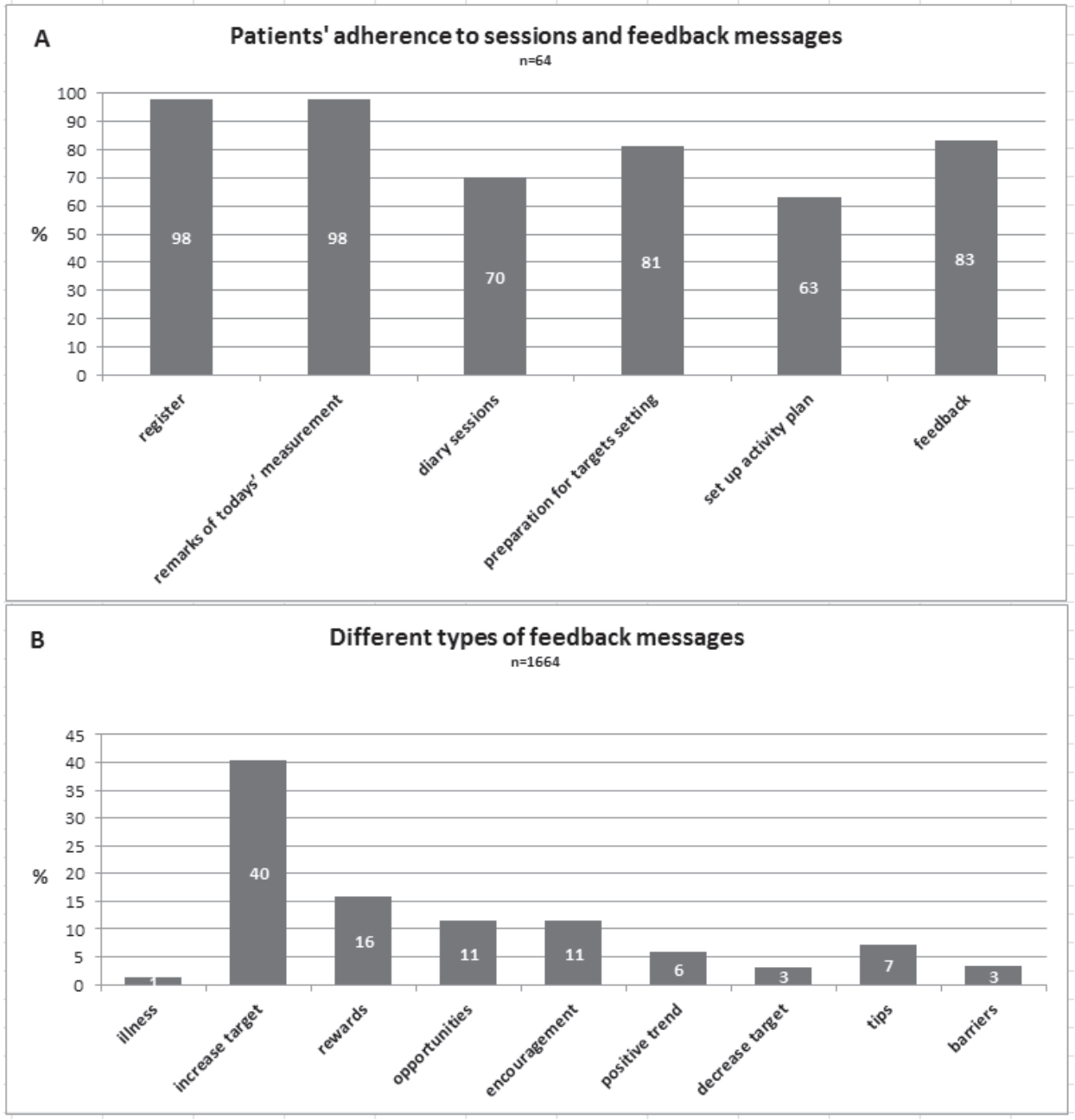

Figure 3 Sessions and feedback messages.

Web application (nurses)

Ninety percent $(n=9)$ of the nurses viewed the results using the web application during consultations, and $50 \%(n=5)$ viewed results in between consultations. The frequency of use varied from rarely, two times in total, to twice a month per patient. Twenty percent 
$(n=2)$ of the nurses experienced problems in using the web application. Some nurses mentioned that PA results from certain patients were not available or not representative for the activity of the patient. In general, the activity levels were lower than the patients expected.

\section{Satisfaction with the intervention (dose received (satisfaction))}

Eighty-four percent of the patients in group 1 and 70\% in group 2 were satisfied with the intervention. Patients in both groups indicated that they were more physically active, more conscious about being physical active, more motivated to exercise, and that their physical fitness improved. Patients from group 1 were significantly more positive about the intervention than those in group $2\left(\chi^{2}(2, n=113)=11.17, p=0.004\right)$. Those patients were more explicit in their positive opinions about the intervention, specifically about the fact that use of the tool was fun; it led to greater awareness and more discipline.

Patients from group 2 appreciated the extra coaching by the nurse. The opinions on how effective the total programme was, differed significantly between the groups; $75 \%$ of the patients in group 1 and $46 \%$ of patients in group 2 thought that PA levels were improved $\left(\chi^{2}(2, n=110)=8.18, p=0.004\right)$. Among the nurses $80 \%$ in group 1 and $33 \%$ in group 2 thought the intervention was effective and led to higher levels of physical activity. According to group 1 patients, the effectiveness was specifically attributed to the tool $(n=11)$. The nurses in group 2 indicated that participants became more aware of the need for PA, but they doubted the actual change. Suggestions for improvement were very diverse; patients and nurses in group 1 mainly came up with improvements for the tool, patients and nurses in group 2 suggested group sessions, more consultations or supervised training sessions.

\section{Satisfaction with the SSP}

Eighty-seven percent (group 1: $88 \%$ and group 2: $86 \%$ ) of the patients and 74\% (group 1: 90\% and group 2: 56\%) of the nurses were satisfied with the number of the consultations. The majority were also satisfied with the content of the consultations, but there was a significant difference between the groups $\left(\chi^{2}(2, N=19)=6.37, p=0.040\right)$. Group 1 nurses were mainly satisfied or neutral (90\%), while the view of group 2 nurses varied more; $67 \%$ were satisfied and $33 \%$ were dissatisfied with the content of consultations. In group 1, the nurses indicated that the intervention encouraged people to be more physically active because they had more insight into their exercise habits, and nurses liked the possibility to monitor their patients through the use of the web application. The nurses from group 2 mainly emphasised the importance of attention to PA. However, they indicated that the execution of the intervention was rather timeconsuming, especially in the beginning. 


\section{Satisfaction with the tool}

Although most patients in group 1 were positive about the tool, the functioning could be improved. Also the nurses indicated that the technical problems were demotivating. Daily wear of the activity monitor was not a problem. Only $9 \%(n=3)$ of the patients were not satisfied with the sessions; however, the feedback messages could be improved according to $32 \%(n=19)$ of the patients. Suggestions for improvement of the feedback messages were: more variation $(n=9)$, send less frequently $(n=6)$, and to make them more personalised $(n=6)$.

\section{Discussion}

The aim of this process evaluation of the It's LiFe! RCT was to examine the reach, the implementation, and satisfaction with the interventions: the counselling protocol (SSP), which was delivered in both groups; and the use of the tool, which was used only by patients in group 1.

It proved extremely difficult to find enough practices and patients to participate in the study. Ten times the number of practices had to be approached until a sufficient number of practices agreed, and within the practices, almost three times the number of patients.

Within the participating patients, the execution of the intervention was adequate; in $83 \%$ of the patients, the number and planning of the consultations were carried out as intended, and patients remembered the different aspects of the Five A's model as being part of the conversation in $71 \%$ of the cases. Of all components of the SSP, discussing the risks of a sedentary lifestyle and the preferences of patients for specific activities were carried out the best, and discussing the tips for local activities the least. In addition, a large proportion of the patients in group 1 used the tool as intended; no less than $88 \%$ used it until the end of the intervention period, in spite of the technical problems which occurred in more than $50 \%$ of cases.

Patients and nurses in group 1 were more satisfied about the intervention; nurses indicated that the self-monitoring encouraged people to be more physically active and they liked the possibility to monitor their patients through the use of the web application. Surprisingly, the technical problems had little impact on satisfaction; patients from group 1 were significantly more positive about the intervention than those in group 2. There is a possibility that the rapid and adequate service of the helpdesk contributed to this. But it remains highly important to test and retest mobile technology several times before scheduling a large effect study.

The encountered difficulties in finding enough participating practices is in line with the conclusions of a study in which barriers for physical activity promotion in primary care were investigated; identified barriers were a lack of time for health promotion activities, and inadequate practice capacity. ${ }^{33}$ Integrating lifestyle counselling into busy 
daily practice while simultaneously complying with the many other clinical demands remains a challenge. ${ }^{34}$ The difficulty in recruiting sufficient numbers of patients was to some extent related to the fact that one of the inclusion criteria was 'access to the internet', and the fact that the intervention involved the use of a smartphone. However, a recent study revealed that $93 \%$ of Dutch care users have internet access, and $51 \%$ use a smartphone, with $12 \%$ already using self-monitoring devices to access physical activity levels. ${ }^{35}$ Based on these figures it is plausible that these barriers for the adoption of the intervention will decrease in the near future.

Compared to other physical activity promotion interventions in primary care, ${ }^{36}$ a relatively high percentage of the patients received the minimal intervention, especially when compared to exercise referral programmes, in which $80 \%$ of the patients seem to drop out before the end of the programme. ${ }^{37}$ The high adherence rate towards the tool is comparable with other studies, in which interventions using a smartphone also resulted in high adherence rates. ${ }^{38}$

Although a minimum amount of time was spent instructing the nurses in how to perform the intervention and, despite the often busy schedules of the nurses, the execution of the consultations and the Five A's model therein was adequate. A study in Scotland revealed that in regular care, practice nurses are likely to recommend patients to take moderate exercise. However, only few correctly describe the current PA recommendations. ${ }^{39}$ These conclusions were confirmed by a study observing the Five A's in PA counselling in the United States, which revealed that interventions to increase skills of nurses in exploring ambivalence and readiness to change, as well as improve explicit mention of recommended guidelines for PA are needed. ${ }^{40}$ In this trial the instruction charts per consultation contributed to the performing of the intervention. They contained clear directions about the guidelines and how to apply motivational interviewing, and the nurses appreciated this.

Since 2010 a broad innovative approach to disease management based on care standards was implemented in primary care in the Netherlands. The Chronic Care Model is the basis of this disease management approach, which aims to transform primary care towards a more proactive care, not only focussed on acute illness but also on maintaining health and preventing or postponing disease. ${ }^{41}$ Self-management support and patient empowerment are essential elements of this approach, in which the practice nurse fulfils an important role. ${ }^{42}$ It is already known that patients with COPD or type 2 diabetes are generally positive about the self-management support by practice nurses, ${ }^{43}$ this is in line with the satisfaction with the SSP we measured in this study. Regarding the use of the tool, patients mentioned similar experiences as described in the SMART MOVE study, ${ }^{44}$ such as more awareness and knowledge, control and focus, and confidence. Patients were satisfied with the automatic tracking of physical activity, the goal setting and visual feedback provided by the tool. Behaviour change theories lay at the basis of the development of the tool, this adds to a better satisfaction of apps to promote exercise. $^{45}$ 
Although this is an extensive process evaluation executed among all intervention practices of the It's LiFe! trial, it has several limitations. At first, the Self-management Support Programme was only evaluated subjectively through the opinion of nurses and patients. No measurements were available to test these elements more objectively, for example through audio-recording of the consultations. Secondly, although the response rate to the patient's evaluation questionnaire was high, it was an assessment of a group of patients who were likely to be rather positive about the intervention, because the majority of dropouts did not return the process evaluation questionnaire. However, a strength of the study was its pragmatic approach; the trial was conducted in very diverse practices with patients with two types of chronic conditions, although ethnic minority groups were represented by only a few patients. Therefore it is unknown how translatable the intervention might be for diverse ethnic and linguistic populations. Further strengths of this study are that it is based on an existing theoretical framework: Saunders' model for process evaluation. In addition, all individual aspects of the Five A's model were evaluated. Also the mixed methods approach is considered as positive; especially the fact that qualitative content analyses were carried out by two researchers independently and before effect outcomes of the RCT were known to them.

As a next step, a more flexible approach towards the intervention should be investigated. The strict inclusion process of the RCT led to exclusion of people who were not yet motivated. In daily practice the practice nurse can execute the intervention in a more gradual way, adapted to the stage of change of the patient, which may lead to a higher reach. In the end, using mobile technology will change the way consultations for monitoring chronic conditions in primary care will take place. In the Netherlands at the moment, reimbursement is based on regular scheduled consultations rather than on supporting self-management by continuous monitoring of conditions in collaboration with patients. The organisational and cost aspects should be further investigated when implementing on a larger scale.

\section{Conclusion}

The results of this process evaluation provide a clear distinction between patients' satisfaction of physical activity counselling with and without the use of the tool, although in both cases patients valued the attention to physical activity promotion by the PN. Patients who used the complementary tool were more positive about their physical activity improvement, despite the fact that technical problems frequently occurred. The results of the trial confirmed this positive impression. ${ }^{46}$ The execution of the consultations, based on the Five A's model, was adequate, although some nurses struggled to fit the extra consultations into their busy daily practice. 


\section{Acknowledgements}

The authors thank all patients, PNs and GP's who participated in this study, Babette van Doorn and April Boessen for their help recruiting general practices and Claudia Valentijn and Arjan Hageman for their work at the helpdesk. Furthermore, Eveline Habets is acknowledged for her help in conducting and transcribing the interviews, and April Boessen is acknowledged for her help with the data entry.

The project was funded by ZonMw (the Netherlands Organisation for Health Research and Development).

\section{References}

1. World Health Organization. Global health risks : mortality and burden of disease attributable to selected major risks. Geneva, Switzerland: World Health Organization; 2009.

2. World Health Organization. Global recommendations on physical activity for health. 2010; http://www.who.int/dietphysicalactivity/factsheet_recommendations/en/. Last accessed February 20, 2015.

3. World Health Organization. Global action plan for the prevention and control of noncommunicable diseases 2013-2020. Geneva, Switzerland: World Health Organization; 2013.

4. Khan KM, Weiler R, Blair SN. Prescribing exercise in primary care. BMJ. 2011;343:d4141.

5. Meriwether RA, Lee JA, Lafleur AS, Wiseman P. Physical activity counseling. Am Fam Physician. Apr 15 2008;77(8):1129-1136.

6. Peterson JA. Get moving! Physical activity counseling in primary care. J Am Acad Nurse Pract. Jul 2007;19(7):349-357.

7. Strath SJ, Kaminsky LA, Ainsworth BE, et al. Guide to the assessment of physical activity: Clinical and research applications: a scientific statement from the American Heart Association. Circulation. Nov 12 2013;128(20):2259-2279.

8. Hillsdon M, Foster C, Thorogood M. Interventions for promoting physical activity. Cochrane Database Syst Rev. 2005;25(1).

9. Broekhuizen K, Kroeze W, van Poppel MN, Oenema A, Brug J. A systematic review of randomized controlled trials on the effectiveness of computer-tailored physical activity and dietary behavior promotion programs: an update. Ann Behav Med. Oct 2012;44(2):259-286.

10. Foster C, Richards J, Thorogood M, Hillsdon M. Remote and web 2.0 interventions for promoting physical activity. Cochrane Database Syst Rev. Sep 30 2013;9:CD010395.

11. Marcus BH, Ciccolo JT, Sciamanna CN. Using electronic/computer interventions to promote physical activity. Br J Sports Med. Feb 2009;43(2):102-105.

12. van der Weegen $S$, Verwey R, Spreeuwenberg M, Tange $H$, van der Weijden $T$, de Witte L. The Development of a Mobile Monitoring and Feedback Tool to Stimulate Physical Activity of People With a Chronic Disease in Primary Care: A User-Centered Design. JMIR Mhealth Uhealth. 2013;1(2):e8.

13. Verwey $R$, van der Weegen $S$, Tange $H$, Spreeuwenberg $M$, van der Weijden $T$, de Witte L. Get moving: the practice nurse is watching you! A case study of the user-centred design process and testing of a webbased coaching system to stimulate the physical activity of chronically ill patients in primary care. Inform Prim Care. 2012;20(4):289-298.

14. van der Weegen S, Verwey R, Tange HJ, Spreeuwenberg MD, de Witte LP. Usability testing of a monitoring and feedback tool to stimulate physical activity. Patient Prefer Adherence. 2014;8:311-322. 
15. Poulson D, Ashby M, Richardson S. User fit: a practical handbook on user-centred design for Assistive Technology. European Commission, DG XIII, Telematics applications for the integration of the disabled and elderly. 1996.

16. Verwey R, van der Weegen S, Spreeuwenberg M, Tange H, van der Weijden T, de Witte L. Upgrading physical activity counselling in primary care in the Netherlands. Health Promot Int. 2014;1:11.

17. Verwey R, van der Weegen S, Spreeuwenberg M, Tange H, van der Weijden T, de Witte L. A pilot study of a tool to stimulate physical activity in patients with COPD or type 2 diabetes in primary care. $J$ Telemed Telecare. 2014 2014;20(1):29-34.

18. Verwey R, van der Weegen S, Spreeuwenberg M, Tange H, van der Weijden T, de Witte L. A monitoring and feedback tool embedded in a counselling protocol to increase physical activity of patients with COPD or type 2 diabetes in primary care: study protocol of a three-arm cluster randomised controlled trial. BMC Fam Pract. 2014;15(1):93.

19. Craig P, Dieppe P, Macintyre S, Michie S, Nazareth I, Petticrew M. Developing and evaluating complex interventions: the new Medical Research Council guidance. Int J Nurs Stud. May 2013;50(5):587-592.

20. Grol R, Wensing M, Eccles M, Davis D. Improving patient care : the implementation of change in health care. 2013; http://onlinelibrary.wiley.com/book/10.1002/9781118525975. Last accessed February 20, 2015.

21. Hasson H. Systematic evaluation of implementation fidelity of complex interventions in health and social care. Implement Sci. 2010;5(1).

22. Hawe $P$, Shiell A, Riley T. Complex interventions: how "out of control" can a randomised controlled trial be? BMJ. Jun 26 2004;328(7455):1561-1563.

23. Linnan $L$, Stecckler A. Proces evaluation for public health interventions and research: an overview. Proces Evaluation for Public health Interventions and Research. San Francisco: Jossey-Bass; 2002:1-23.

24. Oakley A, Strange V, Bonell C, Allen E, Stephenson J, Team RS. Process evaluation in randomised controlled trials of complex interventions. BMJ (Clinical research ed.). 2006;332(7538):413-416.

25. Saunders R. Developing a process-evaluation plan for assessing health promotion program implementation: a how-to guide. Health Promot Pract. 2005;6(2):134 - 147.

26. NISB. 30minutenbewegen beweegtest. [30 minutes movement activities test] 2010; http://www.30minutenbewegen.nl/home-ik-wil-bewegen/meten-weten/beweegtest.html. Last accessed February 20, 2015.

27. Glasgow RE, Emont S, Miller DC. Assessing delivery of the five "As"for patient-centered counseling. Health Promot Int. 2006;21(3):245-255.

28. Whitlock EP, Orleans CT, Pender N, Allan J. Evaluating primary care behavioral counseling interventions: an evidence-based approach. Am J Prev Med. May 2002;22(4):267-284.

29. Wendel-Vos G, Schuit A, Saris W, Kromhout D. Reproducibility and relative validity of the short questionnaire to assess health-enhancing physical activity. J Clin Epidemiol. 2003;56(12):1163 - 1169.

30. NISB. Sportief bewegen met een chronische longaandoening. 2012 Archived at: http://www. webcitation.org/6PDsk5Av1.

31. NISB. Sportief bewegen met diabetes mellitus. 2012. Archived at: http://www.webcitation.org/6PDsgeCvk.

32. Audrey S, Holliday J, Parry-Langdon N, Campbell R. Meeting the challenges of implementing process evaluation within randomized controlled trials: the example of ASSIST (A Stop Smoking in Schools Trial). Health Educ Res. Jun 2006;21(3):366-377.

33. Josyula LK, Lyle RM. Barriers in the Implementation of a Physical Activity Intervention in Primary Care Settings: Lessons Learned. Health Promot Pract. Jun 272011.

34. Noordman J, Koopmans B, Korevaar JC, van der Weijden T, van Dulmen S. Exploring lifestyle counselling in routine primary care consultations: the professionals' role. Fam Pract. Jun 2013;30(3):332-340.

35. Krijgsman J, Peeters J, Burghouts A, et al. Op naar meerwaarde! eHealth-monitor 2014. 2014. http://www.nictiz.nl/page/eHealth/eHealth-monitor. Last accessed February 20, 2015.

36. Bull FC, Milton KE. A process evaluation of a "physical activity pathway" in the primary care setting. BMC Public Health. 2010;10:463. 
37. Gidlow C, Johnston LH, Crone D, James D. Attendance of exercise referral schemes in the UK: a systematic review. Health Educ J. 2005;64(2):168-186.

38. Carter MC, Burley VJ, Nykjaer C, Cade JE. Adherence to a Smartphone Application for Weight Loss Compared to Website and Paper Diary: Pilot Randomized Controlled Trial. J Med Internet Res. 2013;15(4):e32.

39. Douglas F, van Teijlingen E, Torrance N, Fearn P, Kerr A, Meloni S. Promoting physical activity in primary care settings: health visitors' and practice nurses' views and experiences. J Adv Nurs. Jul 2006;55(2):159168.

40. Carroll JK, Antognoli E, Flocke SA. Evaluation of physical activity counseling in primary care using direct observation of the 5As. Ann Fam Med. Sep-Oct 2011;9(5):416-422.

41. Wagner EH, Austin BT, Davis C, Hindmarsh M, Schaefer J, Bonomi A. Improving chronic illness care: translating evidence into action. Health affairs (Project Hope). 2001;20(6).

42. Gruijters N. NHG/LHV-Standpunt Het (ondersteunend) team in de huisartsenvoorziening: 2011 [Position paper: the supportive team for general practices: 2011].

https://www.nhg.org/sites/default/files/content/nhg_org/uploads/toekomstvisie_-_nhg-lhvstandpunt._het_ondersteunend_team_in_de_huisartsenvoorziening_juni_2011_.pdf Last accessed February 20, 2015.

43. Heiligers $P$, Noordman J, Korevaar J, et al. Kennisvraag: praktijkondersteuners in de huisartspraktijk (POH's) klaar voor de toekomst? 2012. http://www.nivel.nl/sites/default/files/bestanden/Rapport-kennisvraagpoh-def.pdf. Last accessed February 20, 2015.

44. Casey M, Hayes PS, Glynn F, et al. Patients' experiences of using a smartphone application to increase physical activity: the SMART MOVE qualitative study in primary care. Br J Gen Pract. Aug 2014;64(625):e500-508.

45. Cowan LT, Van Wagenen SA, Brown BA, et al. Apps of steel: are exercise apps providing consumers with realistic expectations?: a content analysis of exercise apps for presence of behavior change theory. Health Educ Behav. Apr 2013;40(2):133-139.

46. van der Weegen S \& Verwey R, Spreeuwenberg M, Tange H, van der Weijden T, de Witte L. It's LiFe! Mobile and Web-Based Monitoring and Feedback Tool Embedded in Primary Care Increases Physical Activity: A Cluster Randomised Controlled Trial. J Med Internet Res 2015;17(7):e184. 

CHAPTER 9

General Discussion 


\section{Introduction}

The objective of this dissertation was to develop, test, validate and evaluate a selfmonitoring and feedback tool, which was embedded in a counselling protocol (the Selfmanagement Support Program) in primary care, to stimulate people with COPD or type 2 diabetes in their self-management to become more active. The following steps were used (Figure 1). First the It's LiFe! tool was developed in close collaboration with patients and care professionals in a user-centred design process. The developed tool consists of a 3D accelerometer (the MOX), a smartphone application, and a website for the patient and the practice nurse. Second, all parts of the tool were evaluated in a usability study in a laboratory setting and in daily living. Third, the MOX and another low cost accelerometer (the Pam) were compared to a 'state of the art' accelerometer (the ActiGraph GT3X). The MOX was part of the intervention to influence the behaviour of participants and the Pam was used in the last part of the research to measure the effect of the intervention on physical activity in an independent way. Finally, the effectiveness of the complete It's LiFe! intervention was investigated in a cluster randomised controlled trial. Alongside the trial, a process evaluation was performed using a mixture of quantitative and qualitative research methods.

This discussion chapter summarises the main findings of the research in this dissertation and reflects on some methodological issues and theoretical considerations. Furthermore, implications for future research, practice and policy are provided.

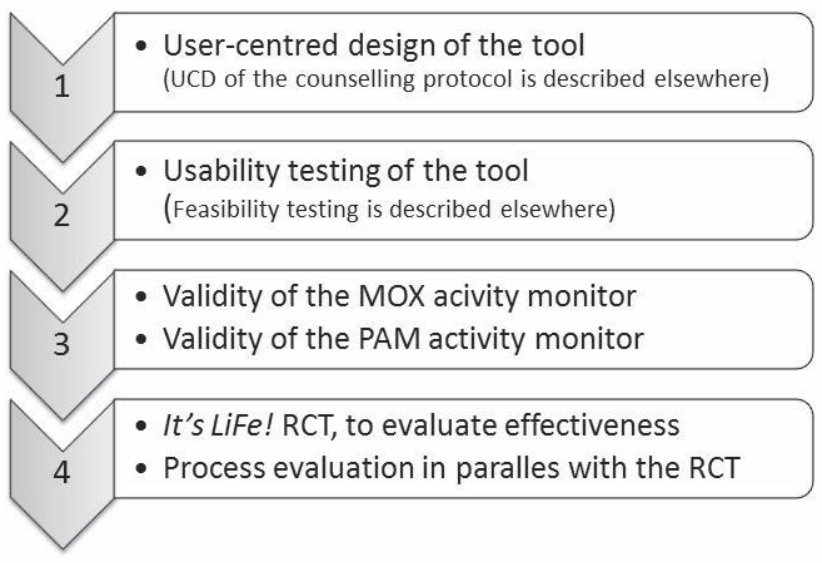

Figure 1 Course of the research presented in this dissertation. 


\section{Main findings}

\section{User-centred design and testing of the monitoring and feedback tool}

The user-centred design process of the monitoring and feedback tool for patients with COPD or type 2 diabetes, described in Chapter 2 , provided valuable insights, which enhanced the fit between the user, technology, and the organization of care. The literature findings and the requirements from end users resulted in a tool with an accelerometer unobtrusively placed on the hip, which is connected to a smartphone with Bluetooth. The smartphone application provides a directly available graphical representation of minutes of activity per day in relation to a personal goal and a historical result overview. In addition, in the menu of the app and on a secured website, patients can complete dialogue sessions (e.g. about barriers and facilitators to become active and make an activity plan) or read feedback messages generated by the system. The aggregated activity data is automatically sent to the practice nurse and discussed in the consultations. An important requirement was the possibility to make annotations to their activity pattern, which is incorporated in the app and the website. ${ }^{1}$

Interviews, questionnaires and observations in a subsequent usability study (Chapter 3) showed the importance of a mixed-method approach, to reveal a wide range of usability issues. The 'expert-based', heuristic evaluation and the 'patient-based' thinkingaloud procedure in the laboratory tests with five patients revealed valuable and detailed feedback on the interfaces and texts. Despite the fact that patients in the laboratory setting were very satisfied with the activity monitor, app and website interfaces, revealed the real-life pilot that the overall satisfaction, according to the SUMIquestionnaire, was low. This lower satisfaction was mainly caused by technological connectivity problems between the different elements of the tool. ${ }^{2}$

\section{Validation of activity monitors}

For the It's LiFe! tool a newly developed accelerometer, 'the MOX', was used as an activity monitor. This accelerometer was developed to be able to accommodate the tool to the user requirements and the other components of the It's LiFe! tool. For this project, the MOX was programmed to send activity counts to the smartphone application of the It's LiFe! tool. In the validation study of the MOX, described in Chapter 4, thresholds for moderate and vigorous intensity physical activity were defined and activity results were compared to activity results from the ActiGraph GT3X. In Chapter 5 another accelerometer, the Pam AM300, was validated. The Pam was used to assess the primary outcome measure of the It's LiFe! Randomised Controlled Trial (RCT). In both studies the activity monitors were validated during treadmill walking and during daily living activities. The measurements on the treadmill revealed that in all accelerometers variance 
between participants increased with speed. This is in line with previous research, which also shows that variance in counts increases with speed. ${ }^{3-5}$ This is not different from the wider variance in $\mathrm{VO}_{2}\left(\mathrm{~mL} \mathrm{~kg}^{-1} \cdot \mathrm{min}^{-1}\right)$ with increasing speed. ${ }^{6,7}$ During treadmill walking both devices had a good correlation with the ActiGraph. However, in the validity study of the Pam, running was investigated in more detail and it was revealed that the ActiGraph did not have a good correlation with speed during treadmill running. This resulted in a lower correlation between the Pam and the ActiGraph. During daily living many data transfer errors occurred and there was no perfect agreement in minutes physical activity between the accelerometers and the reference device (the ActiGraph). Detailed analyses of activity counts in the MOX study demonstrated that misclassification occurred around activity thresholds. ${ }^{8}$

\section{Effects and experiences with the complete It's LiFe! intervention}

The It's LiFe! tool is not a stand-alone device as it has been designed to be integrated in primary care to reach its full potential. In a complementary dissertation the development of the Self-management Support Programme is described. This programme is likewise developed in a User-centred design process, ${ }^{9}$ and its feasibility is together with the tool evaluated in the real-life pilot. ${ }^{10}$ The Self-management Support Programme is based on the $5 \mathrm{~A}^{\prime} \mathrm{s}$ cycle ${ }^{11,12}$ and consists of three to four consultations with the practice nurse. The cluster Randomised Controlled Trial, described in Chapter 7, shows that the complete It's LiFe! intervention, the monitoring and feedback tool embedded in the Selfmanagement Support Programme (SSP), led to a significant improvement of moderate to vigorous physical activity among patients with COPD or type 2 diabetes between 40 and 70 years old in primary care, compared to care as usual. The entire intervention added 12 minutes per day of moderate to vigorous physical activity compared to usual care. Promisingly, the effect was still significant, three months after the intervention. After this period the participants were on average 11 minutes more active than participants in the care as usual group. This study also proved that use of the tool on top of the SSP is more effective than the SSP only. The added value of the tool was an additional 8 minutes of moderate to vigorous physical activity per day. The SSP alone had no significant effect on physical activity compared to usual care. The intervention effect was not so evident for the secondary outcome measures. ${ }^{13}$ The process evaluation (Chapter 8), which was embedded in the trial among the two intervention groups, showed that it was extremely difficult to find enough practices and patients to participate in the study. Ten times the number of practices had to be approached until a sufficient number of practices agreed, and within the practices, almost three times the number of patients was required. The execution of the intervention was adequate; the number and planning of the consultations were carried out as intended (83\%), patients remembered the different aspects of the Five A's model (71\%), and although technical problems occurred fre- 
quently, $88 \%$ of the patients from group 1 indicated to have used the tool until the end of the intervention. From the people who did not use the tool until the end of the intervention, 57\% postulated technical problems as the reason. Explicit attention to physical activity promotion in primary care nursing using the Five A's model was valued by patients as well as nurses. The technical problems had little impact on the satisfaction; patients from group 1 were significantly more positive about the intervention than those in group 2. The complete intervention led to more awareness and discipline. Practice nurses considered the objective measurements a useful addition to their counselling. ${ }^{14}$

\section{Methodological considerations}

This section addresses the most important methodological strengths and limitations of the studies undertaken in this dissertation and potential sources of bias in the research findings.

\section{Benefits and challenges of user-centred design}

The iterative user-centred design process, with a multidisciplinary team, and the usability tests gave valuable insights in the needs and preferences of end users. Most of these insights were incorporated in the design of the tool. Involving end users and stakeholders in drafting the requirements has been shown to be a profitable approach. It improves usability, prevents the inclusion of unnecessary features and maximises the likelihood of a successful adoption. ${ }^{15-17}$ In this project not only interviews, focus groups and tests were held among the end user groups, but also two patient representatives were present at every research meeting. The representatives made sure that all team members were aware of the end users and their context during every step of the process. Furthermore, they supported in practical issues, such as the recruitment of participants and reviewing interview questions. All efforts to involve the end users in the development phase may have led to the high satisfaction rate with the combined intervention and high adherence rate with the tool (88\%) during the trial.

Many technical problems were encountered during the pilot in real life and unfortunately also during the RCT. This led to the drop out of eight participants. Especially connectivity problems between the sensor and the phone and between the phone and the server occurred. This seems to be the consequence of the ambition to develop, test and evaluate technology in a research project in a multidisciplinary team within a certain time frame. Ideally, one more iteration was added to the design process to make the tool more robust. In the It's LiFe! project, different technology companies developed different components of the tool that had to communicate with each other; this proved to be the bottle neck. In addition, requirements of the end users do not always match 
the existing technologies and the different components are not always finished at the same time which delays the progress of projects. Matching the components and incorporating the user requirements in this is money and time consuming. Research projects resulted more often in technology that was not 'mature' enough to be used by the end users. ${ }^{18}$ These challenges could be considered as part of the development process, in which you want to involve end users in every stage and do not want to present them finished technology where no adaptations can be made. However inclusion of participants is very hard and you do not want to cause frustration among them. A negative attitude of the participants to the developed tool can be detrimental for further dissemination in which early adopters should function as ambassadors of your product. On the other hand do developers become so used to the product that they become blind for bugs and they unconsciously create their own 'workarounds'. ${ }^{18}$ An optimal balance between pre-testing by the engineering team and testing by the end users is difficult to find.

In addition, a multidisciplinary team of software developers, health services researchers and end users will enhance the quality and effectiveness of eHealth interventions, as a consequence of the presence of different expertise's. However it is also a challenge to streamline the different values, concepts and expectations from the different fields. A long-term partnership will increase familiarity with each other's terminologies, expectations, theoretical bases, and research methods and ultimately lead to even more benefits from the multidisciplinary collaboration. ${ }^{19}$

\section{Validity of activity monitors}

We used the ActiGraph GT3X as a gold standard to validate the MOX and Pam activity meters. The ActiGraph GT3X is one of the most widely used and validated activity monitors in research environments. ${ }^{20-22}$ Despite this fact, the validation study of the Pam revealed that the ActiGraph did not perform very well during treadmill running. Fifteen participants performed the treadmill running at different speeds (8-10 km/hour). Surprisingly, counts did not continuously increase with speed in the ActiGraph. In six participants, the counts were lower at $10 \mathrm{~km} /$ hour compared to lower speeds. In the literature, the levelling-off effect of the ActiGraph is known as the 'plateau phenomenon'. This is described to be due to limitations in the ActiGraph's frequency filtering characteristics. ${ }^{23-}$ ${ }^{25}$ In addition, many data transfer issues occurred during daily living measurements. The concurrent design in which the MOX and the Pam were compared to the ActiGraph can be questioned. With this design it could not be revealed which accelerometer was responsible for the misclassification of minutes of activity in daily living. The gold standard for the measurement of energy expenditure is doubly labelled water (DLW). ${ }^{26}$ Therefore, accelerometers are validated against DLW. However, a single accelerometer worn around the centre of mass will never have a perfect agreement with energy expenditure, since it does not capture upper body movements. Besides, DLW provides an average 
measure of energy expenditure over a period of one to three weeks and no detailed information about body movement and activity patterns provided by accelerometers. The interest of the studies in this dissertation was actual body movement and not energy expenditure. Nevertheless, the validity of both accelerometers could be refined by a comparison with (video) observation and a test-retest in individual participants to observe variation of measurement in an individual. Furthermore, activity thresholds could be refined by a comparison with indirect calorimetry on a treadmill.

However, both validation studies still provided valuable insights for the It's LiFe! study. Because of the data transfer issues during the validation study of the Pam the data handling protocol of the RCT trial could be adapted accordingly. For the It's LiFe! RCT, Pam b.v. developed a program to detect data transfer issues of Pam data. Furthermore, the activity diaries of the patients were manually checked with the activity data per 15 minutes of the Pam. In the It's LiFe! intervention, the uncertainties caused by the rigid thresholds of the MOX were solved by adding a pre-measurement period. Participants of the intervention used the tool for two weeks and after this period they set a personal goal in collaboration with their care provider. Thus, individual factors that influence activity outcome were taken into account and personal progress was measured after goal setting. In case the pre-measurement revealed that a participant was unable to exceed the general threshold while walking, the practice nurse could lower the threshold. Furthermore, to overcome the fact of underestimation of non-gait related activities the participants had the opportunity to write down remarks in the system to notate extra activities. ${ }^{8}$

\section{Evaluation of the monitoring and feedback tool embedded in a counselling protocol}

Concerning the evaluation of the monitoring and feedback tool two main points will be discussed: the design of the study and the primary outcome measure.

\section{Design}

Simultaneous with the It's LiFe! RCT a process evaluation was conducted to examine the context, implementation, and receipt of the interventions in depth. Process evaluation data was analysed before the effects of the RCT were known to avoid interpretation bias. ${ }^{27}$ However, what should be considered when interpreting process evaluation data is the fact that although intention to treat principles were followed, most dropouts did not complete the questionnaire for the process evaluation because of sickness or because they felt they had too little to say about the intervention. Therefore the process evaluation gives a slightly more positive image of reality.

For the evaluation of healthcare interventions randomised controlled trials are considered as the golden standard. Participants are randomly assigned to the intervention or control group, to minimise allocation bias and effects can be more confidently ascribed to the effects of the intervention rather than to other factors. ${ }^{28}$ In our RCT we 
randomized at cluster level (the family practices) instead of at participant level to avoid contamination. Now, the practice nurses in the control group did not have any additional training and could not unintendedly influence participants in the control group. ${ }^{29} \mathrm{How}$ ever, critics note that RCT's have some drawbacks which makes it difficult to prove effectiveness: 1) the obligation for informed consent can lead to increased effects in control groups; ${ }^{30}$ 2) groups can be too heterogeneous to measure effects; 3 ) often groups are too small to do subgroup analysis to tell which people benefit from the intervention; ${ }^{31} 4$ ) practice changes during trials. Interventions with eHealth, have an additional restraint, because technical developments are so fast that by the time a RCT is designed, organized and executed, the technology is outdated and unappealing. ${ }^{32}$ Furthermore, practice nurses often have limited time for training and mastering the ins and outs of the intervention. In the It's LiFe! trial eight participants per practice participated on average, so by the time the practice nurse was used to the routine of the consultation, the last participant had already left. These facts make it even more exceptional that the RCT showed significant results. In addition, critics to RCT's say that external validity of RCT's is limited. ${ }^{33}$ In the It's LiFe! trial generalizability was enhanced by several factors: 1) general practices of different sizes were included; 2 ) the motivation of participating practice nurses differed; 3 ) the target group was relatively heterogeneous; 4) the integration of the intervention with usual care. One factor that decreased external validity was that only $10 \%$ of the approached practices were willing to participate in the intervention and only $37 \%$ of approached patients agreed to participate. ${ }^{33}$ A common reason from practices for not participating in the study was lack of time. Other studies also reported this reason as a common barrier for more extensive lifestyle counselling. ${ }^{34-36}$ Also the challenge to include enough patients in primary care research is a well-known phenomenon; it is called the 'law of Lasagna'. ${ }^{37}$ in this trial 41 patients were enrolled too few. Despite the fact that recommendations for inclusion were followed such as; prevalent cases were studied, patients were recruited by an invitation letter on stationary from the family practice and approached by a phone call from their practice nurse, ${ }^{37}$.

In addition, the trial would have been more valuable if the follow-up length would be 12 months instead of 9 months. With a follow-up period of 6 months after the end of the intervention, information could have been gathered about maintenance of the increased physical activity levels and adherence toward the use of the tool over longer periods of time. Maintenance of physical activity is important because endured participation is necessary to sustain health benefits. ${ }^{38}$

\section{Outcome measure}

In the RCT, the number of minutes of moderate to vigorous physical activity per day was chosen as primary outcome measure. Physical activity can be measured with questionnaires, devices that measure energy expenditure (doubly labelled water or portable metabolic measurement systems) or activity monitors (pedometers, heart rate monitors, accelerometers or integrated multi-sensor monitors). ${ }^{39,40}$ Questionnaires are a subjec- 
tive measure and provide an overestimation of physical activity. ${ }^{41}$ Energy expenditure is not a direct measure of activity, measurements are time and money consuming and results are difficult to interpret. Consequently it is not a suitable measure for large trials in daily living. Therefore we chose to access physical activity with an activity monitor. We chose a tri-axial accelerometer, as it provides the amount, intensity and duration of movements and is easy to use in daily living. ${ }^{39,40}$ However, measuring physical activity levels with accelerometers also comes with certain issues such as: 1) not capturing upper body movements, cycling, weight bearing and water activities; ${ }^{42,43}$ 2) noncompliance of subjects with wearing the monitor and 3) the possibility of variability between accelerometers of a given model. ${ }^{40}$ The first issue did not lead to bias in the results of the RCT because of randomisation and participants wrote down how many minutes they spent on cycling, swimming and strength training. The second issue was captured; wear time was measured by the tool and subsequently checked with the activity diary. Next, days with less than 8 hours of wearing the tool were omitted from analysis. Moreover, bias due to different wearing times was avoided by correction for wearing time during the analysis. Finally, the third issue was sidestepped by providing the participants the same accelerometer during all three measurements.

As a result of the insights, gained during the validation studies, i.e. the fact that hard thresholds may result in a misinterpretation of the data, an overall physical activity measure without discrete intensity levels was considered. However, such results are difficult to interpret and make it difficult to compare research. ${ }^{44,45}$ Incorporating an additional self-reported measure of physical activity was also taken into consideration. However, this could have resulted in a response shift in group 1 at the second and third measurement period compared to the baseline measurement. The group which received the monitoring and feedback tool as an intervention during four to six months gained inside into their real activity pattern and would most likely have reported their activity level at $\mathrm{t} 1$ and $\mathrm{t} 2$ with less overestimation compared to the other groups and their own baseline measurement.

\section{Theoretical considerations}

The It's LiFe! intervention described in this dissertation focused on improving selfmanagement of chronically ill patients in primary care (secondary disease prevention) regarding their physical activity levels. For this purpose it was chosen to promote daily physical activity instead of explicit referral to an exercise program. Physical activity and exercise are two related concepts that are often used interchangeable. Physical activity is defined as any bodily movement produced by skeletal muscles that results in energy expenditure. ${ }^{46}$ Exercise is a subcategory of physical activity that is planned, structured, and repetitive and has the objective to improve or maintain one or more aspects of physical fitness ${ }^{46,47}$ Although, the It's LiFe! intervention focuses on physical activity and 
not exercise, it is stimulated to plan activities to narrow the gap between intentions and actual behaviour ${ }^{48}$ and if structured training with guidance is preferred, participants are free to take up these activities. In the It's LiFe! intervention participants are stimulated to improve their daily physical activity levels by small changes in their daily routine that will make the adaptations more sustainable and habit formation more likely. In addition, stimulating regular physical activity is more cost-effective than supervised gym-based exercise classes or instructor-led walking programs. ${ }^{49}$ Furthermore, these supervised exercise classes have more barriers for participants such as unwillingness to attend during evening hours, distance to exercise facilities, ${ }^{50}$ an intimidating exercise environment and unfavourable opening hours. ${ }^{51,52}$ Nevertheless, all initiatives to promote physical activity are welcomed in order to counteract the current physical inactivity pandemic. For this challenge an integrated approach is required with changes at an individual, social and cultural, environmental, and policy level. ${ }^{53}$

The Dutch Norm for Physical activity recommends adults to have 30 minutes of physical activity of $\geq 4$ METS (walking with 5-6 km/hour and cycling with $15 \mathrm{~km} /$ hour) on five or more days a week. ${ }^{54}$ To evaluate the It's LiFe! intervention it was chosen to measure the minutes of activity above 3 METS rather than 4 METS because the investigated population consisted of people between 40-70 years old with a chronic disease. For people above fifty five the recommendation is to be active at an intensity of $\geq 3$ METS (walking with 3-4 km/hour and cycling with $10 \mathrm{~km} /$ hour) and for inactive individuals all extra activities are recommended, regardless of duration, frequency, intensity or type. ${ }^{54}$ An age dependent threshold would have made the evaluation of the intervention unnecessarily complicated. There is a tendency to expand physical activity guidelines with the recommendation to interrupt longer periods of sitting. ${ }^{55,56}$ Long periods of sitting have a detrimental effect on glucose metabolism and increase the risk of cardiovascular disease even with sufficient leisure-time physical activity and a healthy waist circumference. ${ }^{56-60}$ In an intervention as It's LiFe! awareness about prolonged sedentary behaviour could be raised by including a vibration of the accelerometer or smartphone after a certain period of sedentary time.

\section{Recommendations}

In the last section of this chapter recommendations for research, practice and policy are discussed.

\section{Future research}

The research in this dissertation reveals that self-monitoring, goal setting and continuous feedback provided by blended care (technology embedded in care) is effective in improving physical activity levels in people with COPD or type 2 diabetes. Because of the 
importance of regular physical activity for everyone, future research should investigate the effects and adherence on the long term, the cost-effectiveness and explore if other target populations could also benefit from the It's LiFe! intervention. For the purpose of the research in this dissertation, people with COPD or type 2 diabetes between 40 and 70 years old were chosen as a target population for the investigation of effectiveness. However, this intervention could be beneficial for all inactive people. Especially for people with chronic conditions who visit the family practice several times a year, such as people with obesity, cardiovascular disease or pre-diabetes. Future research in different target populations should develop a decision aid, incorporating e.g. self-efficacy, stage of change and motivation, to support informed choices who to offer the It's LiFe! intervention. In addition, the intervention does not have to be restricted to the family practice, but could also be of use in other care settings. All care professionals involved in stimulating physical activity could execute the physical activity counselling protocol. For example people with COPD do benefit from extensive pulmonary rehabilitation; it increases exercise capacity, improves quality of life and reduces dyspnoea. ${ }^{61}$ However, once at home it is hard to maintain the achieved results. Incorporating a monitoring and feedback tool during rehabilitation and focus, besides exercise capacity, on regular physical activity behaviour change and overcoming barriers to be active in daily living might improve and extend the benefits of such programs. If the tool should be used continuously or with intervals should be explored.

Above and beyond the opportunities to improve the self-management of physical activity in different target groups and settings, it is worthwhile to investigate if the selfmanagement of other health parameters also improves by adding self-monitoring, goal setting and continuous feedback to the care process. Even though physical activity is an important element in the management of COPD and type 2 diabetes, it is only part of a larger set of self-care behaviours that patients have to face. The monitoring of physical activity could be extended or replaced by other sensors to monitor for example blood sugar levels or oxygen saturation. This could improve the overall self-management of the disease. Self-monitoring of health parameters can create more insight and improve the management of the disease accordingly. For the incorporation of other parameters, sensors should be validated and a new feedback system should be developed with carefully developed cut-off points in a way that the measurements give more insight and does not cause anxiety. When incorporating other health parameters, the communication about the role of the care professional should be even more clear, to avoid false security. It should be clear for the patients that it is a self-management tool and that the care professional is not continuously looking at measurements and cannot intervene accordingly. Another option is to develop a system with well-validated cut-off points should be developed that gives an active sign to the care professional when one should interfere. The continuous measurement of parameters such as physical activity, blood sugar levels, oxygen saturation also creates opportunities for research; it provides a platform for big data from which models can be created to predict relapse for example. 


\section{Practice}

As the effects of the It's LiFe! intervention on physical activity have been demonstrated, it is time to think about the implementation in practice. In daily healthcare practice the intervention provides even more prospects than it did in the research setting, because it can be tailored to a specific setting. As said before, the tool could be of use in the stimulation of physical activity in all people who benefit from more physical activity. Furthermore, the time investment per patient will diminish for the care professional if the intervention becomes part of usual care. Beyond the reach of other patient groups in practice, it is expected that also more patients from the investigated COPD and type 2 diabetes groups will be reached if the intervention is embedded outside a research setting. This is because time-consuming research obligations can be omitted and a more flexible approach to the intervention is possible. The intervention can be offered in a more gradual manner and more adapted to the needs, wishes and stages of change of the individuals. Adapted modes of delivery could be that the patient only commits to participate in the intervention after one or several awareness creating consultations that can be part of usual visits. In addition, the decision to follow the intervention could also be made after the physical activity level is objectively assessed with the tool during a baseline measurement. With these two methods it is also more likely that people participate in the It's LiFe! intervention who actually lack physical activity and do not meet the healthy exercise norm yet. And in daily practice even more tailored decisions can be made such as referral to a physiotherapist if a patient lacks the self-efficacy or exercise capacity to improve its physical activity level in daily living.

However before implementation at a larger scale, the technique should be more stable, without connectivity problems, and affordable. Therefore it is recommended that development teams first improve the current tool and realize an better affordable commercially available version. In addition, if it is proven that self-monitoring and feedback of other health parameters is effective it will be of great value if technical solutions are no longer scattered but integrated with each other. Data from different devices and information systems should be accessible by a self-management portal.

\section{Policy}

That one third of the Dutch population does not meet the standard for healthy exercise, indicates that many people can benefit from an effective physical activity intervention. ${ }^{62}$ The It's LiFe! intervention fits well within the policy of the Dutch Ministry of Health, Welfare and Sport to promote healthy behaviours and promote the self-management of diseases and the formulated ambition to upscale the use of eHealth to support this selfmanagement. One of the goals of the Dutch government is that within five years $75 \%$ of the people with a chronic disease such as diabetes or COPD, is able to carry out health measurements independently, often with remote monitoring by the care provider. ${ }^{63}$ The 
It's LiFe! tool could be one of the technologies to facilitate this policy. However, to reach this goal and to integrate the It's LiFe! intervention in daily practice a few preconditions have to be met:

- Financial reimbursement systems should be adapted to the possibilities of eHealth. Health insurers have already started to incorporate eHealth applications in their contracting policy with caregivers.

- Healthcare providers should gain confidence in eHealth. Nowadays, technology is often seen as cold and distant. The insight that technology can improve care has to grow. The training of care professionals should incorporate knowledge of and experience with eHealth.

- It has to be clear for patients which eHealth applications are available and its significance in their individual situation. An overview of reliable eHealth solutions would help.

- To fully exploit the rapid developments in care technology technical standards should be developed and adopted that make eHealth apps interoperable with electronic patient records and other health information systems. ${ }^{63}$

The promotion of self-management of diseases asks for a different role of the care providers, from a directive role to a more supporting role. This shift in role is essential when eHealth further evolves. The future will most likely be an automated self-management portal that incorporates the information from different sources, like patient records and self-monitoring devices and subsequently presents health data in a meaningful way to the patient. The patient should be in charge of the data and decide whether to share their data with different care providers or informal care givers. Different selfmanagement modules should be offered by the portal. The patient chooses which target behaviour or health parameter he or she wants to work on. This shift from a directive care provider to a directive patient and a care provider as coach, supports patientcentred care as opposed to disease-oriented care.

\section{References}

1. van der Weegen S, Verwey R, Spreeuwenberg M, Tange H, van der Weijden T, de Witte L. The Development of a Mobile Monitoring and Feedback Tool to Stimulate Physical Activity of People with a Chronic Disease in Primary Care: A User-Centered Design. JMIR mhealth and uhealth. 2013;1(2):e8.

2. van der Weegen S, Verwey R, Tange HJ, Spreeuwenberg MD, de Witte LP. Usability testing of a monitoring and feedback tool to stimulate physical activity. Patient Prefer Adherence. 2014;8:311-322.

3. Dowd KP, Harrington DM, Donnelly AE. Criterion and concurrent validity of the activPAL professional physical activity monitor in adolescent females. PLoS One. 2012;7(10):e47633.

4. Esliger DW, Rowlands AV, Hurst TL, Catt M, Murray P, Eston RG. Validation of the GENEA Accelerometer. Med Sci Sports Exerc. Jun 2011;43(6):1085-1093.

5. Kaminsky LA, Ozemek C. A comparison of the Actigraph GT1M and GT3X accelerometers under standardized and free-living conditions. Physiological measurement. Nov 2012;33(11):1869-1876. 
6. Trost SG, Way R, Okely AD. Predictive validity of three ActiGraph energy expenditure equations for children. Med Sci Sports Exerc. 2006;38(2):380.

7. Vanhelst J, Hurdiel R, Mikulovic J, et al. Validation of the Vivago Wrist-Worn accelerometer in the assessment of physical activity. BMC Public Health. 2012;12:690.

8. van der Weegen S, Essers H, Spreeuwenberg M, et al. Concurrent Validity of the MOX Activity Monitor Compared to the ActiGraph GT3X. Telemedicine journal and e-health : the official journal of the American Telemedicine Association. Apr 2015;21(4):259-266.

9. Verwey R, van der Weegen S, Spreeuwenberg M, Tange H, van der Weijden T, de Witte L. Upgrading physical activity counselling in primary care in the Netherlands: The systematic development of a Selfmanagement Support Program combined with mobile technology. Health Promot Int. Dec 232014.

10. Verwey R, van der Weegen S, Spreeuwenberg M, Tange H, van der Weijden T, de Witte L. A pilot study of a tool to stimulate physical activity in patients with COPD or type 2 diabetes in primary care. Journal of Telemedicine and Telecare. Jan 2014;20(1):29-34.

11. Glasgow RE, Emont S, Miller DC. Assessing delivery of the five 'As' for patient-centered counselling. Health Promot Int. Sep 2006;21(3):245-255.

12. Whitlock EP, Orleans CT, Pender N, Allan J. Evaluating primary care behavioural counselling interventions: an evidence-based approach. Am J Prev Med. May 2002;22(4):267-284.

13. van der Weegen S \& Verwey R, Spreeuwenberg M, Tange H, van der Weijden T, de Witte L. It's LiFe! Mobile and Web-Based Monitoring and Feedback Tool Embedded in Primary Care Increases Physical Activity: A Cluster Randomised Controlled Trial. J Med Internet Res 2015;17(7):e184.

14. Verwey R \& van der Weegen S, Spreeuwenberg M, Tange H, van der Weijden T, de Witte L. Process evaluation of physical activity counselling with and without the use of mobile technology: a mixed methods study. Int J Nurs Stud. submitted.

15. Kujala S. User involvement: a review of the benefits and challenges. Behaviour \& information technology. 2003;22(1):1-16.

16. Shah SG, Robinson I. Benefits of and barriers to involving users in medical device technology development and evaluation. Int J Technol Assess Health Care. Winter 2007;23(1):131-137.

17. Abras C, Maloney-Krichmar D, Preece J. User-centered design. Thousand Oaks: Sage; 2004.

18. Vermeulen J, Verwey R, Hochstenbach LM, van der Weegen S, Man YP, de Witte LP. Experiences of multidisciplinary development team members during user-centered design of telecare products and services: a qualitative study. J Med Internet Res. 2014;16(5):e124.

19. Pagliari C. Design and evaluation in eHealth: challenges and implications for an interdisciplinary field. $J$ Med Internet Res. 2007;9(2):e15.

20. Hanggi JM, Phillips LR, Rowlands AV. Validation of the GT3X ActiGraph in children and comparison with the GT1M ActiGraph. Journal of science and medicine in sport / Sports Medicine Australia. Jan 2013;16(1):4044.

21. Vanhelst J, Mikulovic J, Bui-Xuan G, et al. Comparison of two ActiGraph accelerometer generations in the assessment of physical activity in free living conditions. BMC Res Notes. 2012;5:187.

22. Sasaki JE, John D, Freedson PS. Validation and comparison of ActiGraph activity monitors. Journal of science and medicine in sport / Sports Medicine Australia. Sep 2011;14(5):411-416.

23. John D, Miller R, Kozey-Keadle S, Caldwell G, Freedson P. Biomechanical examination of the 'plateau phenomenon' in ActiGraph vertical activity counts. Physiological measurement. Feb 2012;33(2):219-230.

24. Rowlands AV, Stone MR, Eston RG. Influence of speed and step frequency during walking and running on motion sensor output. Med Sci Sports Exerc. Apr 2007;39(4):716-727.

25. Fudge BW, Wilson J, Easton C, et al. Estimation of oxygen uptake during fast running using accelerometry and heart rate. Med Sci Sports Exerc. Jan 2007;39(1):192-198.

26. Plasqui G, Westerterp KR. Physical activity assessment with accelerometers: an evaluation against doubly labeled water. Obesity. Oct 2007;15(10):2371-2379.

27. Oakley A, Strange V, Bonell C, Allen E, Stephenson J, Team RS. Process evaluation in randomised controlled trials of complex interventions. BMJ (Clinical research ed.). 2006;332(7538):413-416. 
28. Eccles M, Grimshaw J, Campbell M, Ramsay C. Research designs for studies evaluating the effectiveness of change and improvement strategies. Qual Saf Health Care. Feb 2003;12(1):47-52.

29. Delgado-Rodriguez M, Llorca J. Bias. J Epidemiol Community Health. Aug 2004;58(8):635-641.

30. Kaptchuk TJ. The double-blind, randomized, placebo-controlled trial: gold standard or golden calf? J Clin Epidemiol. 2001;54(6):541-549.

31. Clay RA. More than one way to measure. Randomized clinical trials have their place, but critics argue that researchers would get better results if they also embraced other methodologies. 2010; Volume 41:http://www.apa.org/monitor/2010/09/trials.aspx. Accessed February 3 2015, 2015.

32. Baker TB, Gustafson DH, Shah D. How can research keep up with eHealth? Ten strategies for increasing the timeliness and usefulness of eHealth research. J Med Internet Res. 2014;16(2):e36.

33. Rothwell PM. External validity of randomised controlled trials: "to whom do the results of this trial apply?". Lancet. Jan 1-7 2005;365(9453):82-93.

34. Jansink R, Braspenning J, van der Weijden T, Elwyn G, Grol R. Primary care nurses struggle with lifestyle counselling in diabetes care: a qualitative analysis. BMC Fam Pract. 2010;11:41.

35. Swinburn BA, Walter LG, Arroll B, Tilyard MW, Russell DG. Green prescriptions: attitudes and perceptions of general practitioners towards prescribing exercise. Br J Gen Pract. Sep 1997;47(422):567-569.

36. Helmink JH, Kremers SP, Van Boekel LC, Van Brussel-Visser FN, Preller L, De Vries NK. The BeweegKuur programme: a qualitative study of promoting and impeding factors for successful implementation of a primary health care lifestyle intervention for overweight and obese people. Fam Pract. Apr 2012;29 Suppl 1:i68-i74.

37. van der Wouden JC, Blankenstein AH, Huibers MJ, van der Windt DA, Stalman WA, Verhagen AP. Survey among 78 studies showed that Lasagna's law holds in Dutch primary care research. J Clin Epidemiol. Aug 2007;60(8):819-824.

38. Marcus BH, Dubbert PM, Forsyth LH, et al. Physical activity behaviour change: issues in adoption and maintenance. Health Psychol. Jan 2000;19(1 Suppl):32-41.

39. Van Remoortel H, Giavedoni S, Raste $Y$, et al. Validity of activity monitors in health and chronic disease: a systematic review. International Journal of Behavioural Nutrition and Physical Activity. 2012;9(1):84.

40. Casaburi R. Activity monitoring in assessing activities of daily living. COPD. Sep 2007;4(3):251-255.

41. Hagstromer M, Oja P, Sjostrom M. Physical activity and inactivity in an adult population assessed by accelerometry. Med Sci Sports Exerc. Sep 2007;39(9):1502-1508.

42. Trost SG, Mclver KL, Pate RR. Conducting accelerometer-based activity assessments in field-based research. Med Sci Sports Exerc. Nov 2005;37(11 Suppl):S531-543.

43. Leenders NY, Sherman WM, Nagaraja HN. Energy expenditure estimated by accelerometry and doubly labeled water: do they agree? Med Sci Sports Exerc. Dec 2006;38(12):2165-2172.

44. Bailey DP, Boddy LM, Savory LA, Denton SJ, Kerr CJ. Choice of activity-intensity classification thresholds impacts upon accelerometer-assessed physical activity-health relationships in children. PLoS One. 2013;8(2):e57101.

45. Ekelund $\mathrm{U}$, Tomkinson $\mathrm{G}$, Armstrong N. What proportion of youth are physically active? Measurement issues, levels and recent time trends. Br J Sports Med. Sep 2011;45(11):859-865.

46. Caspersen CJ, Powell KE, Christenson GM. Physical activity, exercise, and physical fitness: definitions and distinctions for health-related research. Public Health Rep. Mar-Apr 1985;100(2):126-131.

47. Tudor-Locke C, Henderson KA, Wilcox S, Cooper RS, Durstine JL, Ainsworth BE. In their own voices: definitions and interpretations of physical activity. Womens Health Issues. Sep-Oct 2003;13(5):194-199.

48. Sniehotta FF, Scholz U, Schwarzer R. Bridging the intention-behaviour gap: Planning, self-efficacy, and action control in the adoption and maintenance of physical exercise. Psychol Health. 2005/04/01 2005;20(2):143-160.

49. Garrett S, Elley CR, Rose SB, O'Dea D, Lawton BA, Dowell AC. Are physical activity interventions in primary care and the community cost-effective? A systematic review of the evidence. Br J Gen Pract. Mar 2011;61(584):e125-133.

50. Schmidt M, Absalah S, Stronks K. Wat beweegt de deelnemers? Een evaluatie van het project 'Bewegen Op Recept'in Den Haag. Academisch Medisch Centrum. 2006. 
51. Williams NH. "The wise, for cure, on exercise depend": physical activity interventions in primary care in Wales. BrJ Sports Med. Feb 2009;43(2):106-108.

52. Helmink JH, Meis JJ, de Weerdt I, Visser FN, de Vries NK, Kremers SP. Development and implementation of a lifestyle intervention to promote physical activity and healthy diet in the Dutch general practice setting: the BeweegKuur programme. Int J Behav Nutr Phys Act. 2010;7:49.

53. Kohl HW, 3rd, Craig CL, Lambert EV, et al. The pandemic of physical inactivity: global action for public health. Lancet. Jul 21 2012;380(9838):294-305.

54. Wendel-Vos G. Normen lichamelijke (in)activiteit. Volksgezondheid Toekomst Verkenning, Nationaal Kompas Volksgezondheid 2014;

http://www. nationaalkompas.nl/gezondheidsdeterminanten/leefstij//lichamelijke-activiteit/normen-vanlichamelijke-in-activiteit/. Accessed 21 July, 2014.

55. Franklin BA. Health implications of low cardiorespiratory fitness, too little exercise, and too much sitting time: changing paradigms and perceptions. Am J Health Promot. Mar-Apr 2011;25(4):exi-v.

56. Hamilton MT, Healy GN, Dunstan DW, Zderic TW, Owen N. Too Little Exercise and Too Much Sitting: Inactivity Physiology and the Need for New Recommendations on Sedentary Behaviour. Curr Cardiovasc Risk Rep. Jul 2008;2(4):292-298.

57. Morris JN, Heady JA, Raffle PA, Roberts CG, Parks JW. Coronary heart-disease and physical activity of work. Lancet. Nov 28 1953;265(6796):1111-1120; concl.

58. Taylor HL, Klepetar E, Keys A, Parlin W, Blackburn H, Puchner T. Death rates among physically active and sedentary employees of the railroad industry. Am J Public Health Nations Health. Oct 1962;52:1697-1707.

59. Dunstan DW, Salmon J, Owen N, et al. Associations of TV viewing and physical activity with the metabolic syndrome in Australian adults. Diabetologia. Nov 2005;48(11):2254-2261.

60. Dunstan DW, Salmon J, Healy GN, et al. Association of television viewing with fasting and 2-h postchallenge plasma glucose levels in adults without diagnosed diabetes. Diabetes care. Mar 2007;30(3):516-522.

61. Spruit MA, Singh SJ, Garvey C, et al. An official American Thoracic Society/European Respiratory Society statement: key concepts and advances in pulmonary rehabilitation. Am J Respir Crit Care Med. Oct 15 2013;188(8):e13-64.

62. Wendel-Vos G. Hoeveel mensen zijn voldoende lichamelijk actief? Volksgezondheid Toekomest Verkenning, Nationaal Kompas Volksgezondheid 2014;

http://www. nationaalkompas.nl/gezondheidsdeterminanten/leefstijl/lichamelijke-activiteit/hoeveelmensen-zijn-voldoende-lichamelijk-actief/. Accessed April 2015, 2015.

63. Schippers El, van Rijn MJ. Kamerbrief: Betreft e-Health en zorgverbetering. In: Volksgezondheid WeS, ed. Den Haag: Ministerie van Volksgezondheid, Welzijn en Sport; 2014. 


\section{CHAPTER 10 \\ Valorisation}




\section{Introduction}

The research in this dissertation resulted in an effective blended mobile health intervention executed by practice nurses to stimulate physical activity. The intervention consists of a monitoring and feedback tool, an associated coaching system and a counselling protocol. More research is needed to evaluate the effects of this intervention on a larger scale and its cost-effectiveness. However, there are already relevant insights gained during the user-centred design and evaluation of the intervention which are of importance for the value-creation for the different stakeholders involved. Therefore this chapter focusses on emerging opportunities for valorisation that could be taken on the basis of the research presented in this dissertation. Furthermore, this chapter also describes which actions have already been taken to disseminate the knowledge gained in this research. The following definition of 'valorisation' is assumed: The process of valuecreation out of knowledge, by making this knowledge suitable and available for economic or societal utilization and to translate this into high-potential products, services, processes and industrial activity. It concerns the value that can be created through the transfer of scientific knowledge gained during the It's LiFe! project; not only commercializing the monitoring and feedback tool and the coaching system, but also the transfer of acquired knowledge in order to carry out the intervention.

\section{Relevance}

Worldwide many people are not sufficiently active. This is a major problem since physical inactivity has major health effects. According to the World Health Organisation insufficient physical activity is one of the ten leading risk factors for death worldwide and a key risk factor for non-communicable diseases, such as diabetes, cancer and cardiovascular disease. Therefore a lot of initiatives are undertaken to encourage people to become more active, such as national campaigns and initiatives at school, at work and in the neighbourhood. Also primary care providers try to stimulate physical activity of patients. The It's LiFe! intervention supports people with COPD or diabetes type 2 to become more active. More generally, the results of the studies of this dissertation indicate that guidance by a care provider can be reinforced by daily monitoring, feedback and goal setting.

\section{Target groups}

For the following target groups the results of the It's LiFe! project are valuable.

\section{Patients}

In the studies presented in this dissertation the focus was on people with COPD or type 2 diabetes, aged between 40 and 70 years, but there is actually no need to set a maximum 
age to the target group. The most important non-age-related condition is that the patient is motivated to change and in the possession of a smartphone. As the conditions of people with COPD and type 2 diabetes are very diverse, it is to be expected that the intervention could be beneficial for all people who visit the practice nurse regularly and experience barriers to become more physically active. One could even think about using it as a preventive tool for chronic conditions to guide people in general that could benefit from more physical activity regardless their current condition.

\section{Health-care professionals}

In this research the Self-management Support Programme (SSP) was applied by practice nurses. Those nurses were chosen as a mode of delivery since they are explicitly responsible for the promotion of a healthy lifestyle. However, the intervention could also be applied by other care professionals who stimulate a healthy lifestyle, such as physiotherapists, dietitians when treating people with obesity, and general practitioners. Experiences gathered from COPD patients during the user centred design process indicated that especially during rehabilitation programs, which focus on improving exercise capacity, more attention is needed on physical activity in daily living. Patients indicated that extra guidance after a rehabilitation programme is desirable to maintain the benefits. Furthermore, employees from fitness centres, municipalities and people involved in neighbourhood initiatives that focus on stimulating physical activity could use the knowledge gained during the It's LiFe! project.

\section{Industry}

Despite the growing emphasis on eHealth in recent years to improve care processes and outcomes, the scientific evidence of its use often lags behind. This research indicates that automated self-monitoring of physical activity with direct feedback and goal setting embedded in the care process is effective. Companies could use this knowledge in their marketing strategies for self-monitoring devices. Furthermore they can use the knowledge gathered during the user-centred design process to improve their designs and effectuate products which are better adapted to the end users. An insight which could be valuable for future product development is that if self-monitoring takes place and its data is shared with somebody else, the user should have the opportunity to make annotations, to clarify unusual data. Furthermore, especially for the elderly target group, clear instructions and a helpdesk are a necessary condition for acceptation and implementation.

\section{Health insurance companies}

The research presented in this dissertation indicates that self-monitoring embedded in care is an effective intervention to stimulate people to have more physical activity. If the results endure over a longer period of time, this might result in health benefits which will eventually lead to a healthier population, less complications and thus reduces health 
cost which makes it attractive for insurance companies to offer it to their customers. Especially, if the intervention will be implemented as a preventive method to avoid the onset of chronic disease, this would be profitable. With this in mind, it would also be worthwhile to consider providing the intervention in a modified form at work or at school to anybody at risk of an inactive lifestyle.

\section{Activities/products}

Besides the main products developed in this project, the monitoring and feedback tool, the associated coaching system and the Self-management Support Program, other products were developed and activities were undertaken to disseminate the gained knowledge.

\section{Patients}

All study participants and other persons in the It's LiFe! network received two newsletters per year about the progress and the results of the project. Those letters were also available on a website. ${ }^{2}$ Furthermore, the involved companies posted information about the intervention on their websites. ${ }^{3,4}$ During the study period the following products were developed to inform the patients in the study. Patients randomised in the tool group had access to a special website $^{5}$ with information about physical activity and about the use of the tool. At the end of the trial, all participating patients received an overview of their physical activity data. They also received the PAM accelerometer, which they could use optionally in order to continue (group 1) or start (group 2 and 3) with self-monitoring of their daily activity. During the project, the patient representatives acted as ambassador, but further dissemination of knowledge could be done by bringing the results to the attention of other COPD or diabetes type 2 patients through the regular information channels of the patient associations.

\section{Health-care professionals}

Participating health-care professionals were informed about the results of the pilot and the trial directly after the studies in a meeting. Furthermore, several articles were posted in professional journals for nurses, general practitioners and physical therapists. In addition, the results of the studies were announced on (inter)national conferences, which were attended by various researchers, companies and health professionals involved in eHealth and chronic care. In addition, it is important that the end results of the project, which are currently described in English-language scientific journals, will also be published in Dutch professional journals.

The importance of an active lifestyle and how to encourage this should be a standard part of the education of healthcare professionals. Some study results have already been described in a newsletter of the professional association of nurses V\&VN VZI (nurses and healthcare informatics), ${ }^{6}$ but the adapted five $A^{\prime}$ s model for physical activity 
counselling, expanded with the use of the monitoring and feedback tool could also be of interest for practise nurses who are not acquainted with eHealth interventions. The consultation cards, designed to support the practice nurses in how to perform the consultations, are a ready- to- use instrument in the implementation of the intervention on a larger scale. In addition, the knowledge gained in this project will be made available through EIZT, the Centre of Expertise for Innovative Care and Technology of Zuyd University of Applied Sciences. ${ }^{7}$ At this centre, teachers/researchers are working together to give 'technology in care' a more explicit place in the curricula of the various study programs of the Faculty of Health.

\section{Innovation}

The It's LiFe! monitoring and feedback tool is not the only tool which enables an objective measurement of one's physical activity level. Step counters, accelerometers worn at the hip or around the wrist with related applications and Smartphones with integrated accelerometers pursue the same goal. However, the marketing around these devices and apps is mostly targeted at people who are already conscious about a healthy lifestyle and act accordingly (the quantified self). ${ }^{8}$ The innovative aspect about this research is that it was targeted at people with a chronic disease who are difficult to motivate and that it brought together the strengths of new technologies and the coaching role of a care provider. With this combination, people who are normally not triggered by persuasive technology are involved and the coaching role from the care provider is reinforced by providing objective measurements. Daily monitoring and feedback broadens the scope of the consultation room.

\section{Planning and realization}

The research in this dissertation did reveal some suggestions for improvement of the tool such as more tailored and diverse feedback messages, making the tool suitable for the measurement of swimming and cycling, and adding a possibility to share results with peers for extra social support. The latter was waived by participants in the user requirements research, but opted as a suggestion for improvement in the process evaluation of the RCT. The feasibility study and the process evaluation of the RCT among the nurses revealed that nurses want the physical activity results of their patients to be visible in their own electronic health system, rather than on a website. Furthermore, they indicated that they would like to have the possibility to send feedback messages to the patient, rather than call them in between consultations. This would be a valuable option to explore, since it will personalize the feedback for the patient and in this way it can be sent and read whenever possible.

At this moment, the involved companies, Maastricht Instruments and Sananet are working together with a start-up company named 'A.motion' to bring the It's LiFe! tool 
and its services to the market. Their aim is that at the end of 2015 the product and services should be available. They have already launched a pilot project in physiotherapy practices to further explore the possibilities of the It's LiFe! intervention.

In the future more people will monitor their own health variables to get more control over their own health and to be an equal partner in contact with their health professionals. The challenge for system developers and care providers will be to integrate, interpreted and react properly on all these different data. Furthermore, a number of privacy and interoperability issues have to be solved to take full advantage of all technical possibilities.

\section{Referred websites}

1. http://www. netherlandsproteomicscentre.nl/npc/valorisation/what-is-valorisation.html

2. http://www.zuyd.nl/onderzoek/lectoraten/technologieindezorg/projecten/its-life

3. http://www.maastrichtinstruments.nl/portfolio/its-life/

4. http://www.sananet.nl/its-life.html

5. http://www. maastrichtuniversity.nl/web/show/id=6637066/langid=43.

6. http://issuu.com/venvn_vzi/docs/vzi_nieuwsbrief_mei_2014

7. http://www.innovatiesindezorg.eu/

8. http://www.quantifiedself.nl/ 
SUMMARY

SAMENVATTING

DANKWOORD

ABOUT THE AUTHOR

LIST OF PUBLICATIONS 



\section{Summary}

This dissertation reports on six studies of the project named 'Interactive Tool for Selfmanagement through Lifestyle Feedback' (It's LiFe!). The It's LiFe! tool is developed to be used along with counselling from the practice nurse with the aim to support chronically ill patients in achieving a more active lifestyle. The counselling protocol (the Selfmanagement Support Programme (SSP)) was also developed and tested during the It's $\mathrm{LiFe!} \mathrm{Project;} \mathrm{these} \mathrm{results} \mathrm{are} \mathrm{described} \mathrm{in} \mathrm{the} \mathrm{dissertation} \mathrm{of} \mathrm{colleague} \mathrm{Renée} \mathrm{Verwey.}$

The first study of this dissertation reports on the user-centred design of the monitoring and feedback tool (Chapter 2). The second study presents the usability testing of the tool in lab and in real-life by patients (Chapter 3). In the third study the results of the MOX activity monitor, part of the It's LiFe! tool, were compared to the ActiGraph GT3X and activity thresholds were defined (Chapter 4). In a fourth study the Pam AM300, the primary outcome measure of the effect study, was evaluated against the ActiGraph GT3X (Chapter 5). The fifth study, a randomized controlled trial (RCT) executed in 24 family practices, describes the effectiveness of the counselling protocol with and without the use of the tool on physical activity, (exercise) self-efficacy and quality of life (Chapter $6 \&$ 7). Along with the RCT a sixth study was conducted, the process evaluation, to examine the reach and implementation of the It's LiFe! intervention and the satisfaction with the intervention (Chapter $6 \& 8$ ). During the trial and its process evaluation Renée Verwey and Sanne van der Weegen contributed equally to the work, therefore they share the first authorship of Chapter 7 and 8 . These chapters are part of both dissertations.

This dissertation starts with information about the health importance of regular physical activity for healthy adults and for people with a chronic disease (Chapter 1). Many people with a chronic disease experience barriers to become physically active and maintain this behaviour. One of the strategies to increase physical activity in people with a chronic disease is by incorporating support into primary care given by practice nurses. Consequently, support on a daily basis is just as important. Recent developments in technology provide opportunities for this daily support, by facilitating remote selfmonitoring, goal setting and continuous feedback. A combination of both, the support of the practice nurse reinforced by technology, might be valuable.

The first study of this dissertation, presented in Chapter 2, describes the User-centred design process (UCD) of the It's LiFe! tool. The aim of the tool is to stimulate physical activity of people with a chronic disease embedded in primary care practice (by the Selfmanagement Support Programme (SSP)). The leading principle of the intervention was to change behaviour by self-monitoring, goal-setting and feedback. Automated feedback given by the tool and real-life feedback from the practice nurse (according the SSP). In a co-creation, between two companies, two patient representatives and the research 
team, a prototype of the tool was built in three stages. In stage 1, literature was searched to identify end users and context. In stage 2 , the literature, experts and patient representatives were consulted to set up a use case with the general idea of the innovation. In stage 3, individual interviews were held with 15 patients with COPD or type 2 diabetes, 16 care professionals and several experts to identify the end user requirements. Subsequently, the patients were asked to react on the summarized results from the different stages and refine the ideas for a prototype in two focus groups. The developed tool consisted of a tri-axial activity sensor, worn on the hip which is connected by Bluetooth to a smartphone. In an app, quantitative feedback is given about the amount of activity (in minutes a day) and goals reached by means of graphical visualization. Overviews about activity per half an hour, per day, week, and month are provided. In the menu of the app and on a secured website, patients can enter information in individual behaviour change dialogue sessions or read feedback messages generated by the system. The practice nurse can see the results of all patients on a secure webpage and can then discuss the results and set personalized goals in consultation with the patient.

As a last step of the user-centred design process (stage 4) the tool was evaluated in a usability study, described in Chapter 3. The study had four phases: 1) a heuristic evaluation with six technology experts; 2) a usability test in a laboratory by five patients; 3) a pilot in real life wherein 20 patients used the tool embedded in the Selfmanagement Support Programme for 3 months; and 4) a final lab test by five patients. After every phase the tool was updated and improved based on the gained insights. In both lab tests (phases 2 and 4 ) tasks were completed by the participants and qualitative data were collected through a thinking-aloud procedure and video recordings, and quantitative data through questions about task complexity, text comprehensiveness, and readability. In addition, the post-study system usability questionnaire (PSSUQ) was completed for the app and the website. In the pilot test (phase 3), all patients were interviewed three times and the Software Usability Measurement Inventory (SUMI) was completed. After each phase, improvements were made. The main improvement was a refresh button for active data synchronization between activity sensor, app, and server, implemented after connectivity problems in the pilot test. Other improvements were mainly to the layout and text. Results of the PSSUQ indicated that participants in the lab tests were satisfied with the usability of the tool. However, according to the SUMI, participants of the real-life pilot were not very satisfied with the tool. This indicates the importance of a combination of usability tests in a laboratory setting and the use of a system in daily life by the end users.

One of the element of the It's LiFe! tool is the activity monitor. A new activity monitor 'the MOX' was developed to be able to accommodate the tool to the user requirements and the other components of the It's LiFe! tool. Chapter 4, describes the validation of the 
MOX. Thresholds for moderate and vigorous intensity physical activity were defined and activity results were compared to activity results from the ActiGraph GT3X. For this purpose an incremental treadmill protocol was executed by eight healthy adults and ten patients with COPD or type 2 diabetes. In addition, fifteen healthy adults and twelve patients wore the MOX and the ActiGraph during 6-7 days in daily life. In Chapter 5 another accelerometer, the Pam AM300, was validated. The Pam was used to assess the primary outcome measure of the It's LiFe! Clustered- Randomised Controlled Trial (RCT). Results from the Pam were also compared to the ActiGraph GT3X during treadmill walking (by fifteen healthy participants) and during activities in daily living (by ten healthy volunteers and twelve people with COPD or type 2 diabetes). The measurements on the treadmill revealed that in all three accelerometers variance between participants increased with speed. During treadmill walking both devices, the MOX and the Pam, had a good correlation with the ActiGraph. However, in the validity study of the Pam, running was investigated in more detail and it was revealed that the ActiGraph did not have a good correlation with speed during treadmill running. In 6 out of 15 participants counts of the ActiGraph were lower at $10 \mathrm{~km} /$ hour than at 8 or $9 \mathrm{~km} /$ hour. This resulted in a lower correlation between the Pam and the ActiGraph. During daily living many data transfer errors occurred and there was no perfect agreement in minutes physical activity between the accelerometers and the reference the ActiGraph. Detailed analyses of activity counts in the MOX study revealed that misclassification occurred around activity thresholds. For the measurements with the Pam it was not possible to investigate the data in so much detail.

The complete It's LiFe! intervention consists of the tool integrated in the Selfmanagement Support Program. The SSP, is like the tool developed in a User-centred design process, described in the dissertation of Renée Verwey. It is based on the 'Five A's model' (Assess- Advise- Agree- Assist- and Arrange), a counselling model for supporting self-management. The programme consists of three to four behaviour change consultations with a practice nurse spread over a period of four to six months. The practice nurse has access to the activity results of the patients and answers to behaviour change dialogue sessions with the intention of using this information as input for the coaching. During the first consultation the practice nurse assess the current activity pattern with a self-assessment questionnaire, provides information about the risks of a sedentary lifestyle and the benefits of physical activity and provides the tool. During a two week pre-measurement the activity pattern of the patient is assessed by the tool, he/she is asked to complete a behaviour change dialogue session and 6 diary sessions with questions about the exertion and enjoyment of the performed activities. These results are used in the second consultation were the practice nurse and patient set in collaboration an appropriate personal activity goal in minutes per day. The patient is coached by the practice nurse and the tool to make an activity plan with concrete actions to achieve this goal. In addition the patient receives a list of local sports 
activities. In subsequent consultations the practice nurse and patient reflect on the activity results and goal achievement. Automated feedback related to the personal goal is proved continuously by the tool.

Based on the outcomes of the usability study and a three month feasibility study in two general practices, the tool and the Self-management Support Programme were improved. Subsequently a three-armed cluster randomised trial in 24 family practices was set up with 240 participants in total (120 with COPD and 120 with DM2 aged 4070). The study protocol of this study is described in Chapter 6 . The aim of the trial was to evaluate the effectiveness of the SSP with (group 1) and without (group 2) the use of the tool with usual care (group 3). In group 2, the nurses executed the SSP without the tool, with the intention of evaluating the added value of using the tool in combination with the monitor. Recruitment of family practices took place in the southern regions of the Netherlands and randomisation took place at practice level. The intended sample (three arms of eight practices) powered the study to detect a 10-minute difference in moderate and intense physical activity per day between groups 1 and 3. The primary outcome was the number of minutes of moderate to vigorous physical activity per day, measured in all three groups with a physical activity monitor (PAM). These data were analysed by multilevel mixed methods. The secondary outcomes were general - and exercise self-efficacy, quality of life and health status. Outcomes were measured at three time points: at baseline, directly after the intervention (four to six months later) and three months thereafter.

Chapter 7 reports on the effectiveness of the It's LiFe! interventions. The combined intervention (tool + SSP) led to a significant improvement of moderate to vigorous physical activity, compared to usual care. Right after the intervention period, the difference was 12 minutes per day. Three months after the intervention period, this progress was still significant (11 minutes). The trial also proved that use of the tool on top of the SSP is more effective than the SSP only (an additional eight minutes). The SSP alone had no significant effect on physical activity compared to usual care. For the secondary outcome measures the intervention effect was not evident. From these results it can be concluded that the automated self-monitoring and feedback component and/or the fact that the practice nurse was able to see the objectively measured physical activity results were the most powerful elements of the combined intervention.

Chapter 8 reports on the process evaluation of the It's LiFe! trial whose aim was to examine the reach, the implementation, and satisfaction regarding the two main aspects of the intervention: the SSP, which was delivered in both groups, and the use of the tool, which was used only by patients in group 1. It proved extremely difficult to find enough practices and patients to participate in the study. Ten times the number of practices had to be approached until a sufficient number of practices agreed, and within the practices, 
almost three times the number of patients. The drop-out rate during the trial was $17 \%$. The execution of the intervention was adequate; the number and planning of the consultations were carried out as intended (83\%), patients remembered the different aspects of the Five A's model (71\%), and although technical problems occurred frequently, most patients (88\%) indicated that they used the tool until the end of the intervention. Explicit attention to promoting physical activity in primary care nursing using the Five A's model was valued by patients as well as nurses. The technical problems had little impact on the satisfaction; patients from group 1 were significantly more positive about the intervention than those in group 2. The complete intervention led to more awareness and discipline regarding physical activity. Practice nurses considered the objective measurements of the physical activity of their patients a useful addition to their counselling.

In Chapter 9 the main findings of the individual studies are summarized and a reflection on some methodological and theoretical issues which should be taken into consideration when interpreting the results is described. Furthermore, implications for future research, practice and policy are provided.

Finally, Chapter 10 focusses on emerging opportunities for valorization of knowledge gained during the research presented in this dissertation. 



\section{Samenvatting}

Dit proefschrift beschrijft zes van de studies uit het project genaamd 'Interactive Tool for Self-management through Lifestyle Feedback' (It's LiFe!). Het doel van de tool was om fysieke activiteit te stimuleren van mensen met een chronische ziekte. De tool is bedoeld om ingebed te worden in de eerstelijnszorg (hiervoor is tegelijkertijd een Zelfmanagement Ondersteunings Programma (ZOP) ontwikkeld). Het ZOP is ook ontwikkeld en getest tijdens het It's LiFe! project, deze resultaten staan beschreven in het proefschrift van collega Renée Verwey.

De eerste studie van het proefschrift beschrijft het user-centered ontwikkelingsproces van de monitoring- en feedback tool (Hoofdstuk 2). In de tweede studie is de bruikbaarheid van de tool getest door experts en patiënten in een laboratoriumsituatie en in het dagelijks leven (Hoofdstuk 3). In de derde studie zijn de data van de MOXbewegingsmeter, een onderdeel van de It's LiFe! tool, vergeleken met de data van de ActiGraph GT3X en zijn afkappunten gedefinieerd (Hoofdstuk 4). In een vierde studie, is de Pam AM300, de primaire uitkomstmaat van de effectstudie, ook vergeleken met de ActiGraph GT3X (Hoofdstuk 5). In het vijfde onderzoek, in 24 huisartsenpraktijken, is de effectiviteit van het ZOP onderzocht met en zonder gebruik van de tool op lichamelijke activiteit, (exercise) self-efficacy en kwaliteit van leven (Hoofdstuk 6 en 7). Tegelijkertijd met de RCT is er een zesde onderzoek uitgevoerd, de procesevaluatie, om het bereik, de uitvoering en de tevredenheid met deze interventie te onderzoeken (Hoofdstuk 6 en 8). Tijdens de RCT en de procesevaluatie hebben Renée Verwey en Sanne van der Weegen in gelijke mate bijgedragen aan de onderzoeken, daarom delen zij het eerste auteurschap van Hoofdstuk 7 en 8. Deze hoofdstukken maken deel uit van beide proefschriften.

Het proefschrift begint met (Hoofdstuk 1) informatie over het belang van fysieke activiteit voor de gezondheid van gezonde personen en mensen met een chronische ziekte. Veel mensen met een chronische ziekte ervaren barrières om fysiek actief te zijn en dit te blijven. Eén van de strategieën om de mate van fysieke activiteit te verhogen bij mensen met een chronische ziekte is door coaching vanuit de eerstelijnszorg. Deze coaching wordt vooral gegeven door praktijkondersteuners. Het kiezen en toepassen van de juiste strategieën blijft echter een uitdaging. Daarbij hangt het succes van gedragsverandering in fysieke activiteit ook af van de zelfmanagementcapaciteiten van de patiënt. Daardoor is dagelijkse ondersteuning ook belangrijk. Recente technologie ontwikkelingen bieden kansen voor deze dagelijkse ondersteuning, door het faciliteren van zelfmonitoring, doelen stellen en continue feedback. Een combinatie van beide: ondersteuning van de praktijkondersteuner versterkt door het gebruik van technologie lijkt kansrijk. 
In de eerste studie van dit project, weergegeven in Hoofdstuk 2, wordt het usercentered ontwikkelproces van de It's LiFe! tool beschreven. Zoals hierboven beschreven is het doel van de tool om fysieke activiteit te stimuleren van mensen met een chronische ziekte en bedoeld om ingebed te worden in de eerstelijnszorg (hiervoor is tegelijkertijd een Zelfmanagement Ondersteunings Programma (ZOP) ontwikkeld). Het leidende principe van de interventie was om gedrag te veranderen door middel van zelfmonitoring, doelen stellen en feedback. Geautomatiseerde feedback gegeven door de tool en real life feedback door de praktijkondersteuner (volgens het ZOP). In een co-creatie van twee bedrijven, twee patiëntvertegenwoordigers en het onderzoeksteam is een prototype ontwikkeld van de tool in drie fasen. In de eerste fase is er literatuuronderzoek gedaan om de gebruiker en de context te identificeren. In de tweede fase, zijn de literatuur, experts en de patiëntvertegenwoordigers geconsulteerd om een use case op te zetten met het globale idee van de innovatie. In de derde fase zijn 15 patiënten met COPD of diabetes type 2 en 16 zorgverleners geïnterviewd en verschillende expert geconsulteerd om de eisen van de eindgebruikers naar boven te halen en de specifieke eisen te formuleren. Vervolgens zijn de ideeën voor een prototype, op basis van de voorgaande fases, verfijnd in twee focusgroepen met de patiënten. Dit ontwikkelingsproces heeft geresulteerd in een tool bestaande uit een drie-assige bewegingsmeter welke wordt gedragen op de heup. De bewegingsmeter is met Bluetooth verbonden met een smartphone. In een app wordt grafisch weergegeven hoeveel minuten per dag de gebruiker actief is geweest en in hoeverre het doel is bereikt. Tevens zijn er in de app overzichten beschikbaar van de activiteit per half uur, per dag, per week en per maand. In de app en op een beveiligde website kunnen patiënten informatie toevoegen per dag, gedragsveranderingssessies beantwoorden en feedbackberichten vanuit het systeem lezen. De praktijkondersteuner kan de beweegresultaten en de ingevoerde gegevens van de patiënten raadplegen op een beveiligde website. De gegevens dienen als input voor de consulten waarin onder andere een persoonlijk doel wordt opgesteld.

Als laatste stap (fase 4) van het user-centered ontwikkelproces van de tool is een bruikbaarheidsstudie uitgevoerd, deze studie staat beschreven in Hoofdstuk 3. Deze studie bestond uit de volgende vier fases: 1) een heuristische evaluatie met zes technologieexperts; 2) een bruikbaarheidstest in een laboratoriumsetting met vijf patiënten; 3) een pilotstudie waarbij twintig patiënten de tool gebruikten, ingebed in het ZOP, gedurende drie maanden; 4) een afsluitende bruikbaarheidstest in het lab met vijf nieuwe patiënten. Na elke fase werd de tool aangepast en verbeterd op basis van de verworven inzichten. In beide laboratoriumtesten (fase 2 en 4) werden er taken uitgevoerd met de tool door de deelnemers en werden kwalitatieve data verzameld door middel van een 'thinking-aloud' procedure en video-opnames en kwantitatieve data door middel van vragen over de complexiteit van de taken en de begrijpbaarheid en leesbaarheid van de tekst. Tevens werd er een vragenlijst (de Post-Study System Usability Questionnaire (PSSUQ))ingevuld voor de app en de website om de bruikbaarheid van deze onderdelen 
te meten. In de pilot studie (fase 3) zijn de patiënten drie keer geïnterviewd en is er wederom een vragenlijst ingevuld om de bruikbaarheid van de tool te meten. Dit keer met de 'Software Usability Measurement Inventory (SUMI). De grootste verandering die is aangebracht aan de tool op basis van het bruikbaarheidsonderzoek is een knop om actief de data te verversen tussen de bewegingsmeter, de app en de server. Deze verbetering is geïmplementeerd naar aanleiding van verbindingsproblemen in de pilotstudie. Andere verbeteringen omvatte vooral lay-out en tekstuele verbeteringen. De resultaten van de PSSUQ laten zien dat de deelnemers van de lab testen tevreden waren met de bruikbaarheid van de tool, terwijl de SUMI na de pilot laat zien dat mensen niet tevreden waren met de tool. Dit geeft het belang van een combinatie van bruikbaarheidstesten aan. Lab-testen leveren andere informatie dan testen in het dagelijks leven.

Een onderdeel van de It's LiFe! tool is de bewegingsmeter. Ondanks dat er al veel bewegingsmeters op de markt zijn, is er in dit project een nieuwe meter ontwikkeld 'de MOX'. Dit om een tool te hebben die kan worden aangepast aan de gebruikerseisen en de andere componenten van de tool. In Hoofdstuk 4 wordt de validatie van deze MOX beschreven. Er zijn afkappunten gedefinieerd voor matig intensieve en intensieve fysieke activiteit en de resultaten van de MOX zijn vergeleken met de resultaten van een veel gebruikte bewegingsmeter in het onderzoek de ActiGraph GT3X. Hiertoe hebben acht gezonde volwassenen en tien patiënten met COPD of diabetes type 2 op een loopband gelopen met toenemende snelheid. Daarnaast hebben vijftien volwassenen en twaalf patienten de MOX en een ActiGraph gedragen gedurende 6-7 dagen. In de studie, beschreven in Hoofdstuk 5 is een andere bewegingsmeter gevalideerd, de Pam AM300. De Pam is gebruikt als primaire uitkomstmaat in het It's LiFe! geclusterde gerandomiseerde onderzoek. Resultaten van de Pam zijn ook vergeleken met de ActiGraph GT3X tijdens het lopen op een loopband (door vijftien gezonde deelnemers) en tijdens activiteiten in het dagelijks leven (door tien gezonde vrijwilligers en twaalf mensen met COPD of diabetes type 2). De metingen op de loopband openbaarde dat de variantie in resultaten tussen deelnemers bij alle drie de bewegingsmeters groter werd als de snelheid toenam. Tijdens het lopen op de loopband hadden beide bewegingsmeters (de MOX en de Pam) een goede correlatie met de ActiGraph. Echter, in de validatiestudie van de Pam, zijn de resultaten tijdens rennen in meer detail onderzocht en hieruit bleek dat de ActiGraphdata geen goede correlatie heeft met snelheid tijdens rennen. In zes van de vijftien deelnemers waren de counts lager bij $10 \mathrm{~km} / \mathrm{uur}$ dan op 8 of $9 \mathrm{~km} / \mathrm{uur}$. Dit resulteerde in een lagere correlatie tussen de Pam en de ActiGraph tijdens rennen. Tijdens metingen in het dagelijks leven ontstonden veel problemen met de dataoverdracht. Bovendien was er geen perfecte overeenstemming tussen de bewegingsmeters en de referentiebewegingsmeter, de ActiGraph, wat betreft minuten fysieke activiteit per dag. Gedetailleerde analyse van activiteiten counts in de MOX studie liet zien dat misclassificatie (het plaatsen van een minuut in een andere categorie) vooral voorkwam rondom 
de grenzen van een intensiteitscategorie. Voor de Pam was het niet mogelijk om de data in zoveel detail te bekijken.

De complete It's LiFe! interventie bestaat uit de tool geïntegreerd in het Zelfmanagement Ondersteunings Programma (ZOP). Het ZOP is net als de tool ontwikkeld in een iteratief user-centered ontwikkelproces, beschreven in het proefschrift van Renée Verwey. Het is gebaseerd op het $5 \mathrm{~A}$ model (Assess- Advise- Agree- Assist- and Arrange), een counselingmodel voor het ondersteunen van zelfmanagement. Het programma bestaat uit drie tot vier gedragsveranderingsconsulten met de praktijkondersteuner, verspreid over een periode van vier tot zes maanden. De praktijkondersteuner heeft toegang tot de beweeggegevens van de patiënt en tot de antwoorden op gedragsveranderingsdialoogsessies. Deze resultaten dienen als input voor de coachingsconsulten. Tijdens het eerste consult brengt de praktijkondersteuner samen met de patiënt het huidige activiteitenniveau in kaart door middel van een vragenlijst. Verder geeft de praktijkondersteuner meer informatie over de risico's van een inactieve leefstijl en de voordelen van meer fysieke activiteit en de tool wordt aangeboden aan de patiënt. Tijdens een twee weken durende voormeting wordt het activiteitenniveau van de patiënt objectief vastgesteld door de tool, hij/zij wordt gevraagd om gedragsveranderingsdialoog- en zes dagboeksessies in te vullen met vragen over de mate van inspanning en plezier tijdens uitgevoerde activiteiten. Op basis van deze resultaten stellen de praktijkondersteuner en de patiënt gezamenlijk een haalbaar persoonlijk doel in minuten fysieke activiteit per dag. De patiënt wordt tevens gestimuleerd (door de praktijkondersteuner en door een sessie van de tool) om in een actieplan te concretiseren hoe hij/zij dit doel wil gaan behalen. Daarnaast ontvangt de patiënt een lijst met lokale beweegactiviteiten en verenigingen. In de daarop volgende consulten evalueren de patiënt en de praktijkondersteuner de beweegresultaten en de mate waarin het doel is gehaald. Nadat er een doel is ingesteld in het tweede consult worden er tevens geautomatiseerde feedback berichten gegenereerd op basis van de mate waarin doelen worden behaald.

Op basis van de uitkomsten van de bruikbaarheidsstudie en een drie maanden durende feasibility studie in twee huisartsenpraktijken werden de tool, de monitor en het ZOP verbeterd. Vervolgens werd een cluster gerandomiseerd onderzoek (RCT) bij 24 huisartspraktijken opgezet bestaande uit drie groepen met in totaal 240 deelnemers tussen de 40 en 70 jaar (120 met COPD en 120 met DM2). Het studieprotocol van dit onderzoek wordt beschreven in Hoofdstuk 6. Het doel van dit onderzoek was om de effectiviteit van het ZOP met (groep 1) en zonder (groep 2) het gebruik van de tool te evalueren in vergelijking met gebruikelijke zorg (groep 3). In groep 2 voerden de praktijkondersteuners enkel het ZOP uit, met de bedoeling om de toegevoegde waarde van het gebruik van de tool in combinatie met de monitor te evalueren. Werving van huisartspraktijken vond plaats in de zuidelijke regio's van Nederland en er werd gerandomiseerd op praktijkniveau. De beoogde steekproef (drie groepen van acht praktijken) was voldoen- 
de groot om een gemiddeld verschil van 10 minuten in matige en intensieve beweging per dag tussen de groepen 1 en 3 aan te kunnen tonen. De primaire uitkomstmaat was het aantal minuten matige en intensieve beweging per dag, gemeten in alle drie de groepen met behulp van de Pam bewegingsmeter. Deze gegevens werden geanalyseerd middels multilevel mixed methods. De secundaire uitkomstmaten waren algemene selfefficacy, op sport en bewegen gerichte self-efficacy, kwaliteit van leven en gezondheidsstatus. Uitkomsten werden gemeten op drie tijdpunten: bij aanvang, meteen na de interventie (4-6 maanden) en drie maanden na afloop.

In Hoofdstuk 7 wordt over de effectiviteit van de interventies gerapporteerd. De gecombineerde interventie (tool en ZOP) heeft geleid tot een significante verbetering van matige en intensieve beweging in vergelijking met gebruikelijke zorg. Direct na de interventieperiode bleek dit verschil gemiddeld 12 minuten per dag. Drie maanden na de interventieperiode bleek dit verschil nog steeds significant (11 minuten). Uit het onderzoek bleek tevens dat het gebruik van de tool in combinatie met het ZOP effectiever is dan alleen de toepassing van het ZOP (8 extra minuten). Het ZOP alleen had geen significant effect op beweging in vergelijking met de gebruikelijke zorg. De interventie bleek geen significant effect te hebben op de secundaire uitkomstmaten. Uit deze resultaten kan geconcludeerd worden dat het gebruik van de tool een noodzakelijk onderdeel van de interventie is, wil deze effectief zijn.

Hoofdstuk 8 doet verslag van de procesevaluatie van de RCT It's LiFe!, een onderzoek dat tot doel had om het bereik, de uitvoering en de tevredenheid aangaande de twee belangrijkste aspecten van de interventie te onderzoeken: het ZOP, dat werd uitgevoerd in beide groepen en de tool, die enkel gebruikt werd door patiënten in groep 1. Het bleek bijzonder moeilijk om voldoende praktijken en patiënten te vinden die wilden deelnemen aan het onderzoek. Slechts één van de tien uitgenodigde praktijken en één van de drie benaderde patiënten nam deel aan het onderzoek. 17\% Van de patiënten viel uit tijdens het onderzoek. De interventie is in voldoende mate uitgevoerd door de praktijkondersteuners; het aantal en de planning van de consulten verliep zoals bedoeld (83\%), patiënten herinnerden zich de verschillende aspecten van het Vijf A model (71\%) en hoewel technische problemen vaak voorkwamen gaven de meeste patiënten (88\%) aan de tool tot het einde van de interventieperiode te hebben gebruikt. Zowel patiënten als praktijkondersteuners waardeerden het expliciet aandacht besteden aan stimuleren van meer bewegen met behulp van het Vijf A model. De technische problemen hadden weinig invloed op de tevredenheid; patiënten uit groep 1 waren significant positiever over de interventie dan die in groep 2. De volledige interventie leidde tot meer bewustzijn en discipline ten aanzien van lichamelijke activiteit. Praktijkondersteuners vonden inzicht in beweging van hun patiënten, via de objectieve metingen door de tool, een nuttige aanvulling op hun begeleiding. 
In Hoofdstuk 9 zijn de belangrijkste bevindingen van de individuele onderzoeken samengevat en wordt er gereflecteerd op een aantal methodologische en theoretische kanttekeningen welke in acht moeten worden genomen bij de interpretatie van de resultaten. Vervolgens worden er aanbevelingen gedaan voor onderzoek, de praktijk en beleid.

Tenslotte focust Hoofdstuk 10 zich op de mogelijkheden voor de valorisatie van kennis die is opgedaan tijdens de onderzoeken in dit proefschrift. 


\section{Dankwoord}

Woohoo! De stukken zijn af (nou ja bijna dan). Wat ben ik veel mensen dankbaar dat het zover is gekomen.

Allereerst Renée, ik denk dat wij de afgelopen jaren meer contact hebben gehad met elkaar dan met wie dan ook om ons heen. Wat is het fijn geweest om samen successen te kunnen vieren en om samen te balen als iets niet perfect liep. Ik heb bewondering voor je onuitputtelijke ambitie. Je staat vol energie in het werkende leven en bent steeds nieuwe kansen aan het verkennen. Ik ben benieuwd wat je loopbaan allemaal nog brengt. Ik heb op allerlei gebieden veel van je geleerd en stelling 11 staat mede voor onze samenwerking. Doordat we alles samen deden en afstemden duurde sommige zaken wat langer, maar wat mij betreft is elk stuk en het hele project er beter van geworden. If you want to go fast, go alone. If you want to go far, go together.

Dan het team, precies 5 jaar geleden hadden wij het sollicitatiegesprek, de dag dat ik mijn masterthesis inleverde en een dag voordat ik voor 3 maanden vertrok naar ZuidAmerika. Vanuit Peru hadden we telefonisch het tweede gesprek, jullie hoefden alleen te weten of ik meteen kon beginnen als ik terugkwam. En dat wilde ik! Wat ben ik blij dat jullie me de kans hebben gegeven om dit ontzettend leuke project te mogen uitvoeren. Luc bedankt voor de vrijheid, het vertrouwen en de duidelijke lijn van je begeleiding. Ik denk dat menig promovendi jaloers is op een begeleider die van de eerste tot de laatste dag van het traject dezelfde ideeën uitdraagt. Zeker bij een twijfelaar als ik was dit vertrouwen en de duidelijke lijn zeer welkom. Daarbij was je ondanks je drukke agenda altijd bereikbaar. Marieke, copromotor wat is er de afgelopen jaren veel gebeurd. Je bent zelf gepromoveerd, je bent twee kinderen rijker, een rug operatie verder en nu ben je zelfs aangesteld als lector! Het is bijzonder om te zien hoe enthousiast jij wordt als het om cijfers gaat en je gave om die ingewikkelde statistiek begrijpelijk uit te leggen. Huibert, jij hebt me begeleid tijdens mijn masterthesis, je hebt me in contact gebracht met technologie in de zorg en je hebt me aangespoord om op deze vacature te reageren. Bedankt daarvoor en voor alle grondige reacties op onze stukken. Trudy jij adviseerde ons vanaf een afstandje, maar je adviseerde mij ook van heel dichtbij. Je bent een warme vrouw en goede onderzoeker. Je betrokkenheid was erg waardevol. Kenneth tijdens de validatiestudies was ik aan jou overgeleverd ;). We hebben veel discussies gehad over de methode én de resultaten en ondanks dat er meestal al een volgende PhD student op de gang aan het wachten was nam je altijd de tijd om even te vragen hoe het verder ging. Het was leerzaam om ook vanuit een andere vakgroep een kijk op onderzoek te krijgen.

De leden van de beoordelingscommissie: Prof. dr. ir. R.D. Friele, Prof. dr. ir. H.J. Hermens, Prof. dr. S. Kremers, Prof. dr. D. Ruwaard en voorzitter: Prof. dr. J. Muris hartelijk 
bedankt voor de tijd en energie die jullie gestoken hebben in het beoordelen van dit proefschrift. In het bijzonder wil ik Stef Kremers bedanken. Terwijl ik tijdens mijn bachelor nog dacht over promoveren: 'In één van die kamertjes, vier jaar artikelen schrijven, dat ga ik nooit doen!'. Tijdens de Master heb je me met je enthousiasme en humor geïnspireerd om wel te kiezen voor het onderzoek.

Dit proefschrift zou er niet zijn geweest zonder de medewerking van alle huisartsen, praktijkondersteuners, experts en vooral proefpersonen. Sommige proefpersonen die we aan het begin van het project hebben leren kennen hebben zelfs aan meerdere onderzoeken bijgedragen. Jos en Ina jullie in het bijzonder bedankt. Jullie waren tot de trial bij de onderzoeksvergaderingen en zorgden dat wij de eindgebruikers niet uit het oog verloren en hielpen ons bij de werving van deelnemers. Het was heel fijn om veel contact te hebben met ervaringsdeskundigen. De eerlijke, geïnteresseerde, positieve en kritische reacties hielden ons enthousiast en scherp.

April, jij hebt ook een belangrijke bijdrage geleverd aan het It's LiFe! project, je hebt er onder andere voor gezorgd dat tijdens het laatste onderzoek de data van 199 proefpersonen heel precies werd ingevoerd en dat niet één maar drie keer! Heel veel succes met het afronden van je Master! Hans, jij hebt alle data uitgelezen en bewerkbaar gemakt voor de validatiestudies. Wat was dat een puzzel, maar heel fijn dat we veel issues hebben kunnen tackelen voor we aan de RCT begonnen. Willem Bekker, bedankt voor het geheel belangeloos controleren van alle beweegdata (ook 3×199) het was heel fijn dat we ons daar geen zorgen over hoefde te maken. Milosh bedankt voor het samen klaarmaken van de tasjes met handleidingen, telefoons, simkaarten en bewegingsmeters, in je stageweek.

Paolo Nutini, London Grammar, The Foals e.a. bedankt voor de muzikale ondersteuning tijdens het schrijven van de laatste stukken.

Collega's door de goede sfeer op de vakgroep kan ik me niet herinneren dat ik een dag met tegenzin naar het werk ben gegaan. Joan, onze pionier van de 'technologie in de zorg club', jij had de meeste wegen al net voor ons bewandeld en was altijd bereid om ons te voorzien van goed advies. Sparren over het onderzoek en ervaringen uitwisselen over wel of niet werkende technologie kon later ook met Laura en Martine. Het was ontzettend leuk om met jullie, de UM-technologiedames en Gaston, mijn laatste werkweek af te sluiten in Lissabon. Laura, jou kan ik bij meerdere kopjes noemen, technologiecollega, vrimibo-compagnon, fietsmaatje etc etc. Wat hebben we veel gelijkenissen, wat was het fijn om altijd even langs te kunnen lopen en wat ben ik blij dat je me bij wil staan als paranimf. Je bent een hele attente ambitieuze meid, veel geluk met Gaston in jullie nieuwe huis!

Dat langs lopen gold eigenlijk voor heel kamer 0.043. Maike en Susan jullie ook bedankt voor de vele (on)zinnige gesprekken en gezellige lunch-, koffie-, vrimibo-, kerstdi- 
ner- en festivalmomentjes! Beste HSR collega's, de lunchwandelingetjes en gesprekken met jullie waren een fijne afwisseling! Donja met jou heb ik de laatste jaren met veel plezier een kamer en heel veel verhalen gedeeld. Heel veel geluk met Jeroen. Hopelijk vinden jullie een mooie plek in Nederland waar jullie eindelijk samen kunnen wonen, ik kijk uit naar al je toekomstige reisverhalen. Lotte, bij Vilans werk ik met veel plezier met jou samen op verschillende projecten en daarnaast heb je de kaft van dit boekje ontworpen! De andere plaatjes in het proefschrift zijn design technisch totaal onverantwoord; ik kan nog veel van je leren de komende jaren.

Andrea, Claudia, Simon (Master matties!) en Bart, wat was het fijn om meteen na het werk te gaan sporten met jullie! Spinning, club power, fitness, bootcamp en natuurlijk wielrennen, jullie waren (bijna) overal voor in. Heerlijk om alles te geven, je verhaal kwijt te kunnen en daarna samen eten. Andrea, bikkel met je gekke ideeën, 51 km hardlopen in Zwitserland. Respect voor je doorzettingsvermogen en discipline! Claudia, jij hardwerkende super vrouw, jij staat altijd voor iedereen klaar wat fantastisch is om te zien (en te ervaren), maar vergeet jezelf niet! Simon, jij hebt ervoor gezorgd dat ik ook met de oudjes ging Wii-en. Jij meette de veranderingen in balans en ik de ervaringen en eisen voor implementatie. Jij hebt als geen ander de ups en down van de afgelopen jaren meegekregen tijdens het sporten. Super fijn om deze periode af te sluiten met jou als paranimf ernaast. Bart, wij hebben ook heel wat uren samen doorgebracht bij UM-Sport en op de fiets. Jammer dat ik die Limburgse heuvels en je retrofiets moet missen. lone, bedankt voor je positieve energie, de onverwachte briljante avonden, bbq's, concerten en andere feestjes. Alle andere vrienden, het wordt teveel om alle toffe mensen om me heen hier te benoemen, maar ontzettend bedankt voor jullie vriendschap, goede gesprekken, steun, mooie weekendjes, vakanties en feestavonden.

Lieve pa, ma, Joachim en Janneke, een promotietraject staat bekend om de leerzame tijd en de zure appels waar je af en toe doorheen moet bijten. De wet van Murphy is inderdaad verschillende keren voorbij gekomen, maar in vergelijking met wat bij jullie langs kwam in deze jaren was het promoveren één zoete appeltaart. Jullie helpen mij relativeren en zijn mijn inspiratiebron om elke situatie positief te bekijken.

Lieve, lieve Maarten, jij ondersteunt me in alles wat ik doe, zorgt dat ik het beste uit mezelf kan halen en trapt soms op de rem als ik iets teveel wil. Bedankt daarvoor en voor nog zoveel meer. Het jaar met de vele life-events zit er bijna op, zullen we nu een bouwval kopen? Ik kijk in ieder geval uit naar alle avonturen die we nog gaan beleven! 


\section{About the author}

Sanne (J.C.M.) van der Weegen was born on the $25^{\text {th }}$ of November 1986 in Steenbergen, the Netherlands.

After completing her secondary education (VWO) at Mollerlyceum in Bergen op Zoom in 2005, she moved to Maastricht to study General Health Sciences at Maastricht University. In 2008 she obtained her Bachelor degree. A year later she continued with the Master Sports and Physical Activity Interventions at the same university. She graduated cum laude in 2010, with a thesis on the experiences of elderly with the Nintendo Wii and the requirements for implementation in a nursing home.

In October 2010 Sanne started her PhD trajectory under supervision of prof. dr. L.P. de Witte, at CAPHRI, the School for Public Health and Primary Care, at the department of Health Services Research at Maastricht University. Six studies from this trajectory are presented in this thesis.

Since March 2015 she works for Vilans (Utrecht, The Netherlands), centre of expertise for long-term care, at the department of Research and Innovation with a focus on eHealth. 


\section{List of publications}

\section{International journals}

- van der Weegen S \& Verwey R, Spreeuwenberg M, Tange H, van der Weijden T, de Witte L. It's LiFe! Mobile and Web-Based Monitoring and Feedback Tool Embedded in Primary Care Increases Physical Activity: A Cluster Randomised Controlled Trial. J Med Internet Res 2015;17(7):e184

- van der Weegen S, Essers H, Spreeuwenberg M, Verwey R, Tange H, de Witte L, Meijer K. Concurrent Validity of the MOX Activity Monitor Compared to the ActiGraph GT3X. Telemed J E Health. Apr 2015;21(4):259-266.

- Verwey R, van der Weegen S, Spreeuwenberg M, Tange H, van der Weijden T, de Witte L. Upgrading physical activity counselling in primary care in the Netherlands. Health Promotion International. 2014;1:11.

- Vermeulen J, Verwey R, Hochstenbach LM, van der Weegen S, Man YP, de Witte LP. Experiences of multidisciplinary development team members during user-centered design of telecare products and services: a qualitative study. J Med Internet Res. 2014;16(5):e124.

- Verwey R, van der Weegen S, Spreeuwenberg M, Tange H, van der Weijden T, de Witte L. A monitoring and feedback tool embedded in a counselling protocol to increase physical activity of patients with COPD or type 2 diabetes in primary care: study protocol of a three-arm cluster randomised controlled trial. BMC Family Practice. 2014;15(1):93.

- van der Weegen S, Verwey R, Tange HJ, Spreeuwenberg MD, de Witte LP. Usability testing of a monitoring and feedback tool to stimulate physical activity. Patient Prefer Adherence. 2014;8:311-322.

- Verwey R, van der Weegen S, Spreeuwenberg M, Tange H, van der Weijden T, de Witte L. A pilot study of a tool to stimulate physical activity in patients with COPD or type 2 diabetes in primary care. J Telemed Telecare 2014;20(1):29-34.

- van der Weegen S, Verwey R, Spreeuwenberg M, Tange H, van der Weijden T, de Witte L. The Development of a Mobile Monitoring and Feedback Tool to Stimulate Physical Activity of People With a Chronic Disease in Primary Care: A User-Centered Design. JMIR Mhealth Uhealth. 2013;1(2):e8.

- Verwey R, van der Weegen S, Tange H, Spreeuwenberg M, van der Weijden T, de Witte L. Get moving: the practice nurse is watching you! A case study of the usercentred design process and testing of a web-based coaching system to stimulate the physical activity of chronically ill patients in primary care. Inform Prim Care. 2012;20(4):289-298. 


\section{Conference proceedings and abstracts}

- van der Weegen S \& Verwey R, Tange H, Spreeuwenberg M, van der Weijden T, de Witte L. Cluster Randomised Controlled Trial of a Mobile Monitoring and Feedback Tool Embedded in a Counselling Protocol to Stimulate Physical Activity in Chronically III Patients. In M. Hettinga, A. Smedberg, L. Van Gemert-Pijnen, K. Dyb, \& A. G. Ekeland (Eds.), eTELEMED The $7^{\text {th }}$ International Conference on eHealth, Telemedicine, and Social Medicine, Lisboa, Portugal; IARIA. 2015;164-167. (full paper, oral presentation)

*Awarded as 'best conference paper'

- Verwey R \& van der Weegen S, Tange H, Spreeuwenberg M, van der Weijden T, de Witte L. Cluster Randomised Controlled Trial of a Mobile Monitoring and Feedback Tool Embedded in a Counselling Protocol to Stimulate Physical Activity in Chronically III Patients. In M. Hettinga, A. Smedberg, L. Van Gemert-Pijnen, K. Dyb, \& A. G. Ekeland (Eds.), eTELEMED The $7^{\text {th }}$ International Conference on eHealth, Telemedicine, and Social Medicine, Lisboa, Portugal; IARIA. 2015;161-163. (full paper)

- van der Weegen S \& Verwey R \&, Spreeuwenberg M, Tange H, van der Weijden T, de Witte L. It's LiFe! Het gebruik van een model voor monitoring en feedback tool in de eerste lijn om fysieke activiteit te stimuleren: effecten en ervaringen. COPD ketencongres, Utrecht, the Netherlands; 2015. (invited speakers)

- van der Weegen S, Verwey R, Spreeuwenberg M, Tange H, van der Weijden T, de Witte L, A monitoring and feedback tool embedded in primary care stimulates physical activity. Health Care Innovation, Amsterdam, the Netherlands; 2015. (poster presentation)

- Verwey R, van der Weegen S, Tange H, Spreeuwenberg M, van der Weijden T, de Witte L. Technology combined with a counselling protocol to stimulate physical activity of chronically ill patients in primary care. In $12^{\text {th }}$ International conference on Nursing Informatics, Taipei, Taiwan; Stud Health Technol Inform. 2014;201:264-270. (full paper)

- van der Weegen S, Verwey R, Spreeuwenberg M, Tange H, van der Weijden T, de Witte L. Usability testing of a monitoring and feedback tool measuring and stimulating physical activity. Med-e-Tel 2013, Luxemburg; ISfTeH. 2013. (oral presentation)

- van der Weegen S, Essers H, Spreeuwenberg M, Verwey R, Tange H, Meijer K, de Witte L. Validation of the It's LiFe! monitoring and feedback tool. ICAMPAM 2013 International Conference on Ambulatory Monitoring of Physical Activity and Movement, Amherst, Massachusetts, USA; 2013. (poster presentation)

- Verwey R, van der Weegen S, Tange H, Spreeuwenberg M, van der Weijden T, de Witte L. Get moving: the practice nurse is watching you! In L. Van Gemert-Pijnen \& H.C. Ossebaard (Eds.), eTELEMED The $5^{\text {th }}$ International Conference on eHealth, Telemedicine, and Social Medicine, Nice, France; IARIA. 2013 188-193. (full paper)

*Awarded as 'best conference paper' 
- van der Weegen S, Verwey R, Spreeuwenberg M, Tange H, van der Weijden T, de Witte L. User-centred development of a monitoring and feedback tool to stimulate physical activity. International Congress on Telehealth and telecare, London, United Kingdom; International Journal of Integrated Care. 2012;12(Suppl1). (poster presentation)

- Verwey R, van der Weegen S, Tange H, Spreeuwenberg M, van der Weijden T, de Witte L. A monitoring and feedback tool to support patients in achieving a more active lifestyle. In L. van Gemert-Pijnen, H.C. Ossebaard, A. Smedberg, S. Wynchank \& P. Giacomelli (Eds.), eTELEMED The $4^{\text {th }}$ International Conference on eHealth, Telemedicine, and Social Medicine, Valencia, Spain; IARIA. 2012 147-150. (full paper)

- van der Weegen S, Verwey R, Spreeuwenberg M, Tange H, van der Weijden T, de Witte L. It's LiFe!: A Monitoring-and Feedback Tool to Stimulate Physical Activity, Embedded in Primary Care. In F. Grieco, A.J. Spink, O.E. Krips, L.W.S. Loijens, L.P.J.J. Noldus \& P.H. Zimmerman (Eds.) Measuring Behavior, Utrecht, the Netherlands; Noldus Information Technology. 2012:118. (full paper, Invited speaker)

- van der Weegen S, Verwey $R$, Spreeuwenberg M, Tange $H$, van der Weijden $T$, de Witte L. It's LiFe! User-centered design van een monitoring- en feedback systeem om fysieke activiteit te stimuleren van eerstelijns COPD en diabetes type 2 patiënten. Medisch Informatica Congres. Veldhoven, the Netherlands; 2011. (poster presentation) 
Veel mensen met een chronische ziekte, zoals COPD of diabetes type 2 lukt het niet om voldoende te bewegen. Eén van de strategieën om dit te verbeteren is door ondersteuning van een praktijkondersteuner in de huisartsenpraktijk. Succesvolle gedragsverandering hangt echter ook voor een groot deel af van de zelfmanagementcapaciteiten van de patiënt. Technologie kan deze capaciteiten versterken door het faciliteren van zelfmonitoring, het stellen van doelen en het geven van continue feedback. Door de gecombineerde hulp van praktijkondersteuner en technologie moet veel voordeel te behalen zijn. De Universiteit Maastricht heeft daarom in het project It's LiFe! in samenwerking met twee bedrijven (Maastricht Instruments en Sananet), een tool ontwikkeld die gebruikers stimuleert om meer te gaan bewegen. De tool bestaat uit een bewegingsmeter, die draadloos is verbonden met een smartphone en een online coachingsysteem. Via een app is te zien hoeveel minuten er bewogen zijn in relatie tot persoonlijke doelen. Gebruik van de tool is ingebed in een zorgprogramma dat bestaat uit extra consulten bij de praktijkondersteuner. De praktijkondersteuner kan de beweegresultaten van gebruikers van de tool via het coachingsysteem volgen.

In dit proefschrift beschrijft Sanne van der Weegen: a) de ontwikkeling en het testen van de tool in nauwe samenwerking met patiënten en zorgverleners b) de validatie van twee bewegingsmeters en c) de evaluatie van de tool in combinatie met het zorgprogramma in de praktijk. De resultaten van het evaluatieonderzoek bij 24 huisartsenpraktijken wijzen uit dat deze gecombineerde interventie effectief is en door patiënten en praktijkondersteuners gewaardeerd wordt. Dit proefschrift is relevant voor mensen met een chronische aandoening die meer willen bewegen en voor zorgprofessionals die deze patiënten begeleiden. De uitkomsten van het project zijn eveneens van belang voor wetenschappers en beleidsmakers die zich bezig houden met een gezonde leefstijl, het meten van bewegen of geïnteresseerd zijn in de ontwikkeling van een mHealth interventie.

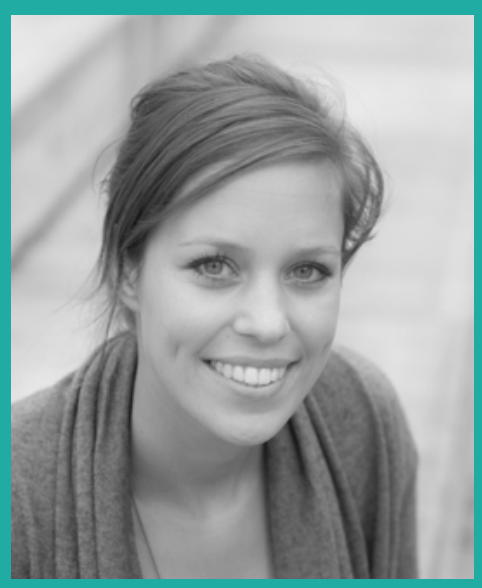

Sanne van der Weegen (1986) heeft vanaf oktober 2010 aan dit proefschrift gewerkt bij de vakgroep Health Services Research binnen CAPHRI School for Public Health and Primary Care aan Maastricht University. 